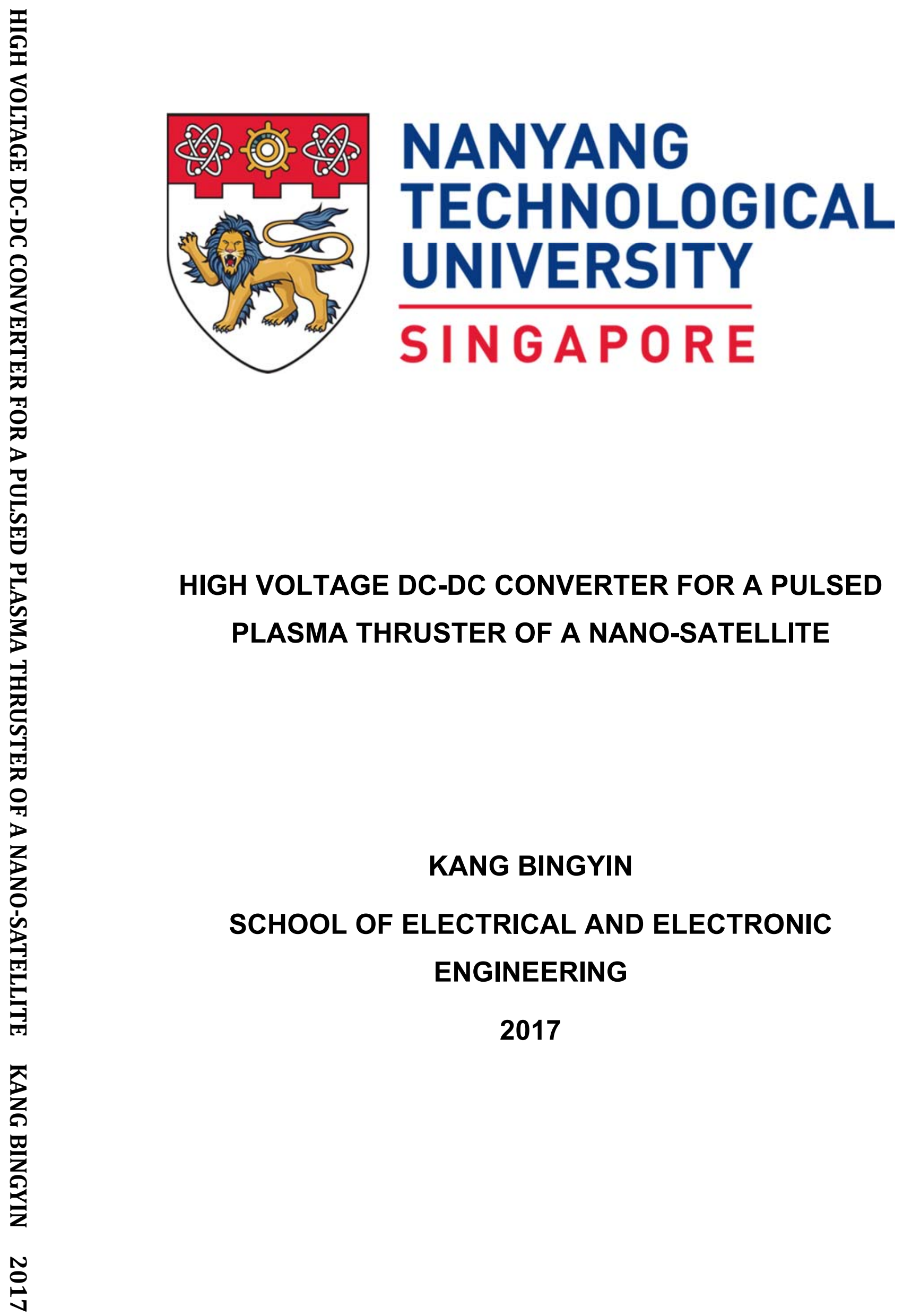




\title{
HIGH VOLTAGE DC-DC CONVERTER FOR A PULSED PLASMA THRUSTER OF A NANO-SATELLITE
}

\author{
KANG BINGYIN
}

School of Electrical and Electronic Engineering

A thesis submitted to the Nanyang Technological University in partial fulfillment of the requirement for the degree of Doctor of Philosophy 


\section{Acknowledgment}

I would first like to express my uppermost gratitude to my supervisor Professor Low Kay Soon for his kind encouragement, patient guidance and continuous support throughout my Ph.D studies. Professor Low has imparted me with the philosophy of doing research. I have learned a lot from him on the academic writing, paper reading, research presentation, idea innovation, etc. His rigorous scholarship is my role model in my future career. I also greatly thank Associate Professor Ali Iftekhar Maswood to supervise my thesis submission.

I would also like to show my sincere thanks to the laboratory executives from Satellite Research Centre (SaRC), Mrs. Chong-Eng Puay Cheng, Mrs. Tham Suet Mei, Mrs. Tan Geok Lan Janet, Mrs. Yap Poh Geok Pamela and Mrs. Low Siew Kheng. They have always been enthusiastic to help me with the administrative matters.

I would like to thank the research fellows and postgraduate students in SaRC, Dr. Tsai Yung-Fu, Dr. Soon Jing Jun, Dr. Tran Quang Vinh, Dr. Htet Aung, Dr. Lee Guo Xiong, Dr. Li Bingxuan and Mr. Han Bo. The discussions with them have inspired me greatly when I am in confusion. I am also greatly thankful to my colleagues in SaRC who have provided friendship and shared technical experience. Their very excellence motivates me to chase a better achievement.

Last but not least, I thank all my friends and family members. Dr. Ye Wanxin and Dr. Zou Qiong have taught me a lot to avoid common mistakes probably met during Ph.D study. My parents have provided their unconditional support to whatever decisions that I have made. They have been working diligently to bring up my elder brother and me. My elder brother took care of my parents during these years when I have been 
studying oversea. I would like to appreciate his contributions to the family as well as his encouragement throughout my study. 


\section{Table of Content}

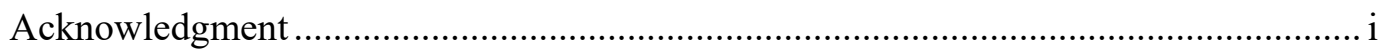

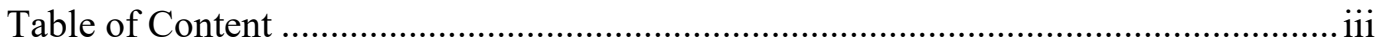

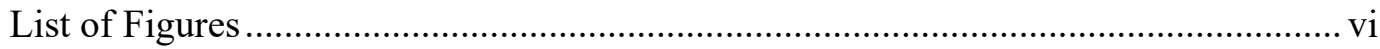

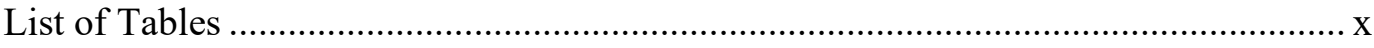

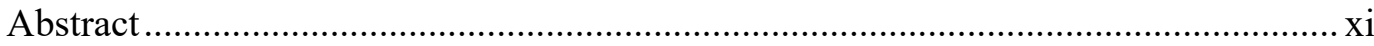

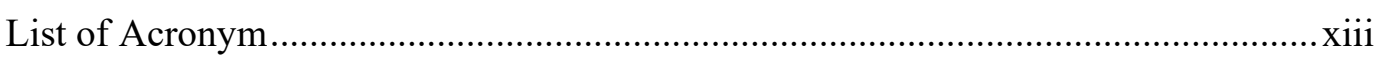

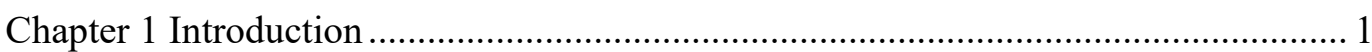

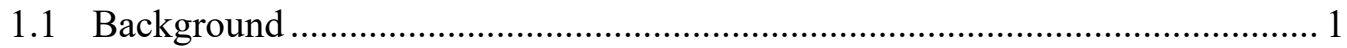

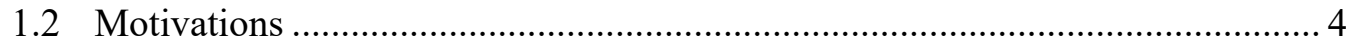

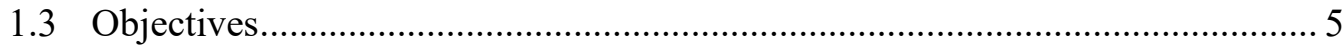

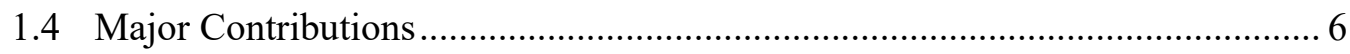

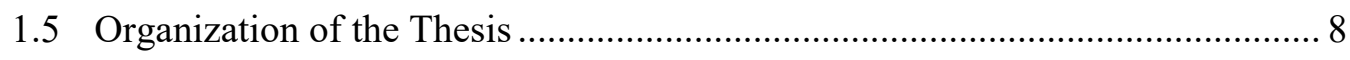

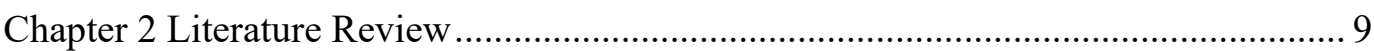

2.1 Candidate Topologies for PPT Igniter .......................................................... 10

2.1.1 Topologies Using Hard-switching ........................................................... 10

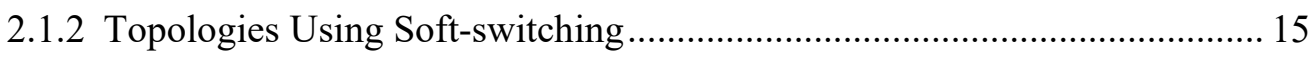

2.2 Candidate Topologies for Capacitor Charging ............................................ 22

2.2.1 Flyback and Forward Converters ........................................................... 22

2.2.2 Resonant Converters ........................................................................... 24

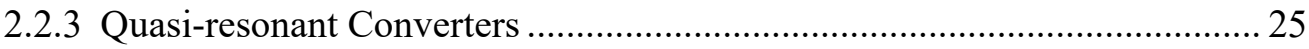

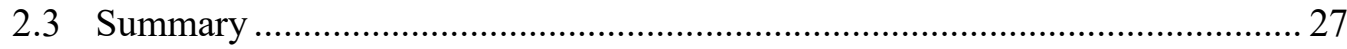

Chapter 3 Pulse Voltage Generation Using a Flyback Circuit with Parasitic Components

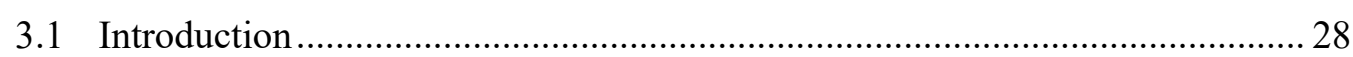

3.2 Flyback Circuit with Parasitic Components................................................... 29

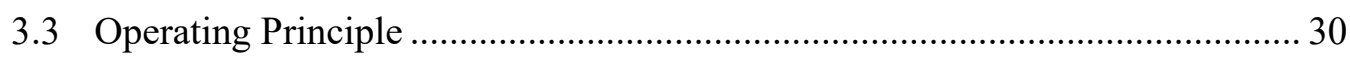

3.4 Benchmarking with Other PPT Igniter Circuits............................................ 34

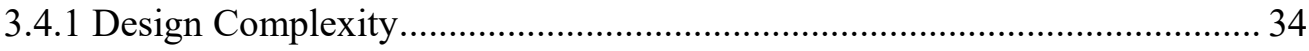

3.4.2 Number of High Voltage Capacitors........................................................ 35

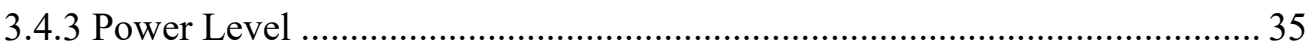

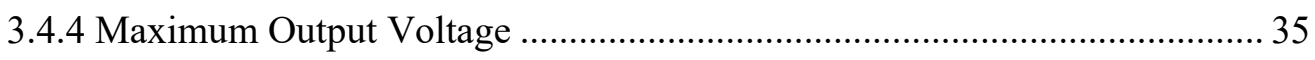

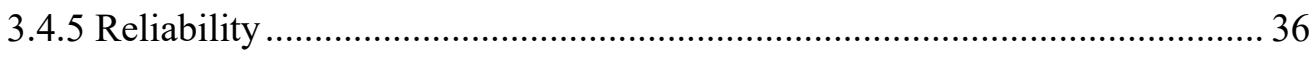

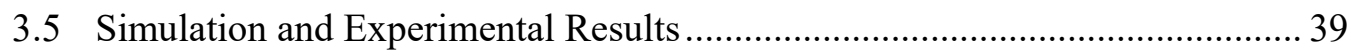

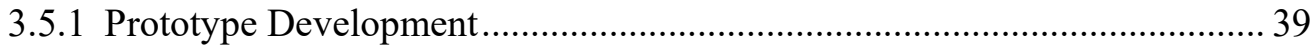

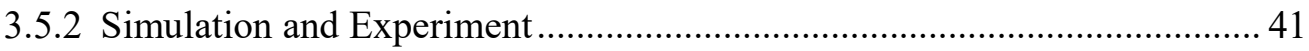




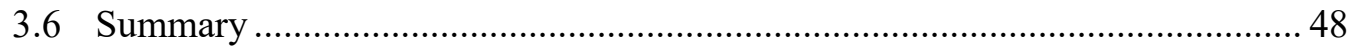

Chapter 4 Single Switch Quasi-Resonant DC-DC Converter ........................................ 49

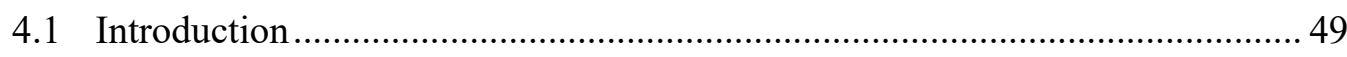

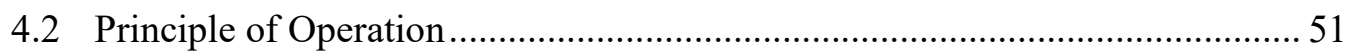

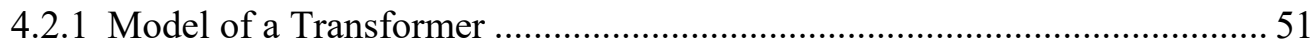

4.2.2 Cockcroft-Walton Voltage Multiplier ....................................................... 52

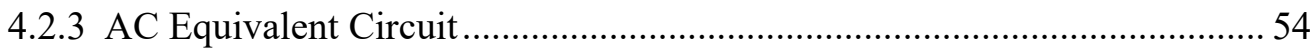

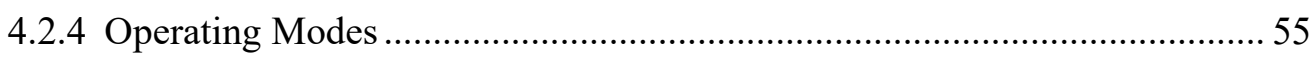

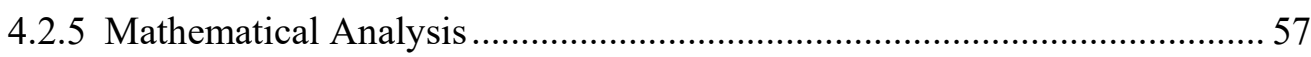

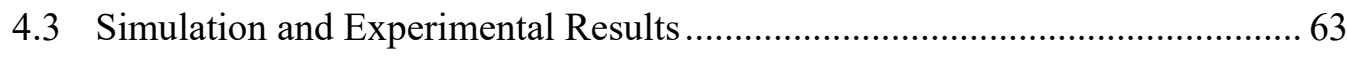

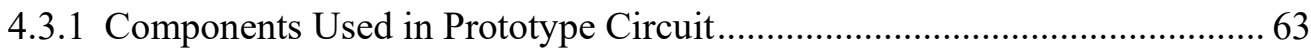

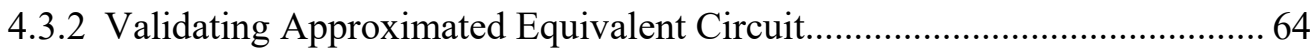

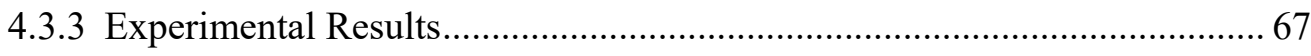

4.4 Comparisons with Existing Resonant Converters.......................................... 76

4.4.1 Number of Switches and Switching Control............................................... 76

4.4.2 Soft-switching and Range of Switching Frequency …………………......... 76

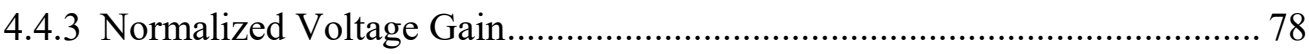

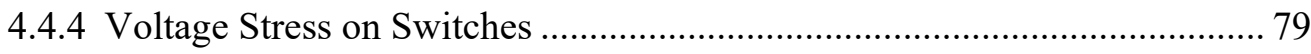

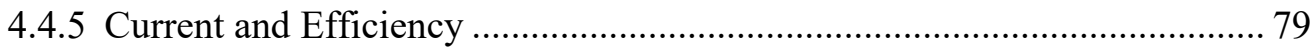

4.5 Comparisons with Circuits Using a Single Switch ........................................... 82

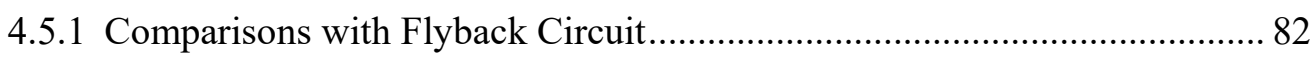

4.5.2 Comparisons with Quasi-resonant Flyback Converter.................................... 83

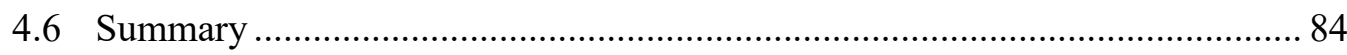

Chapter 5 Quasi-resonant Flyback Converter for Capacitor Charging.......................... 85

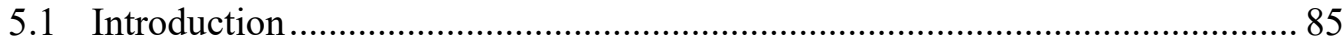

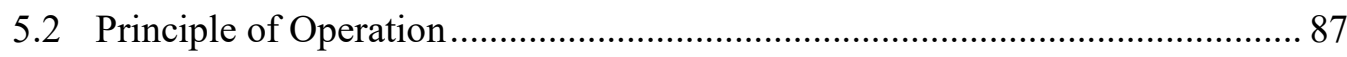

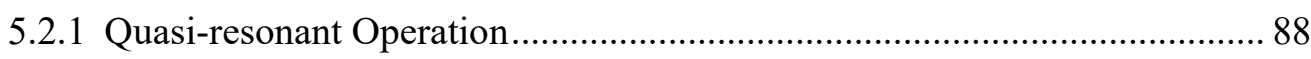

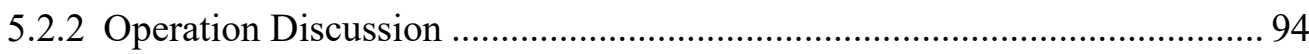

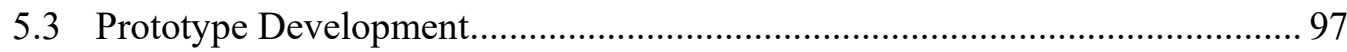

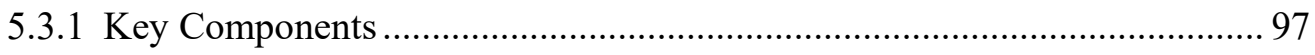

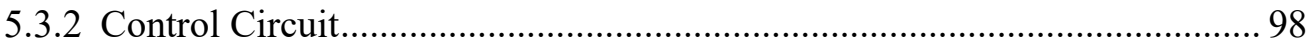

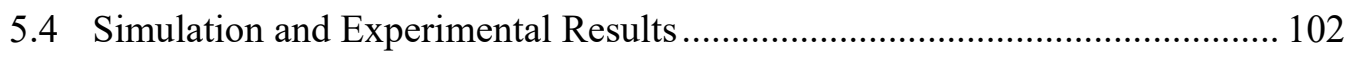

5.4.1 ZVS throughout Capacitor Charging Process ………………………....... 102

5.4.2 Hybrid Switching Control ......................................................................... 113

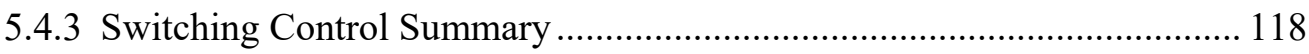

5.5 Comparisons with Conventional Flyback Converter ………………………....... 119

5.5.1 Voltage Stress on Switch......................................................................... 119 


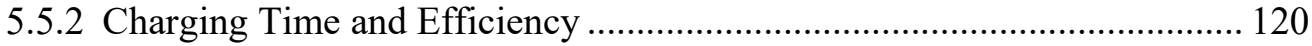

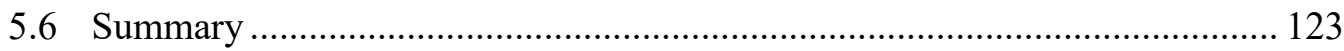

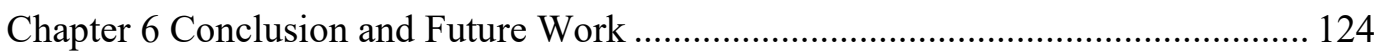

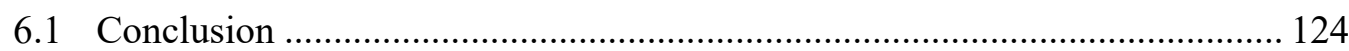

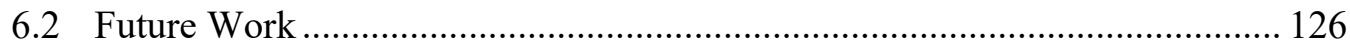

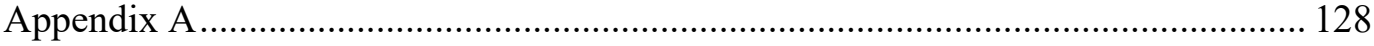

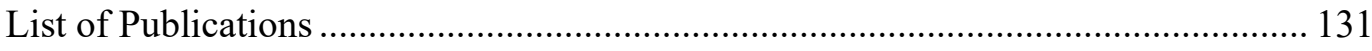

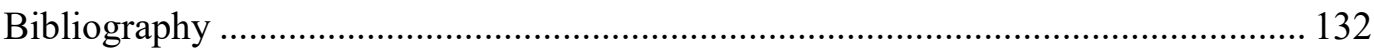




\section{List of Figures}

Fig. 1.1 Block diagram of a pulsed plasma thruster (PPT) ....................................... 3

Fig. 2.1 Switched capacitors: (a) Cockcroft-Walton voltage multiplier (VM), and (b)

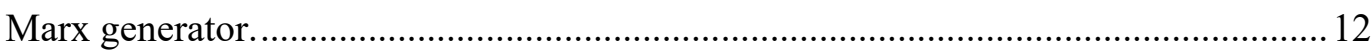

Fig. 2.2 PPT igniter circuit used in (a) satellite Dawgstar (US) [12], (b) satellite STSAT-

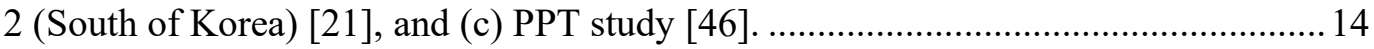

Fig. 2.3 Modular flyback converter with (a) parallel connection, and (b) series

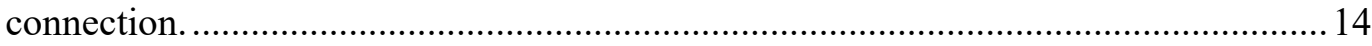

Fig. 2.4 Block diagram of a transformer-based resonant converter............................. 16

Fig. 2.5 Types of resonant tanks: (a) series resonant tank, (b) parallel resonant tank, (c)

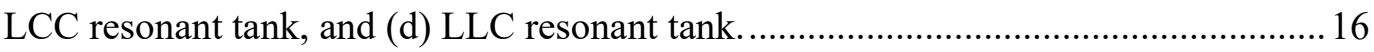

Fig. 2.6 Common AC model of a resonant converter for first harmonic approach (FHA).

Fig. 2.7 (a) Conventional flyback converter, and (b) forward converter. 23

Fig. 2.8 Key waveforms of quasi-resonant flyback converter using valley-voltage-

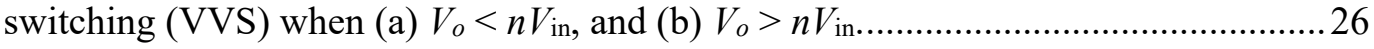

Fig. 3.1 Block diagram of a pulse power supply....................................................28

Fig. 3.2 Flyback circuit (a) without, and (b) with parasitic capacitances.....................30

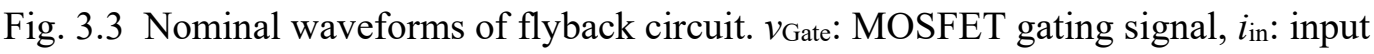
current, $i_{L}$ : current of magnetizing inductance, $v_{p}$ : primary side voltage, $v_{o}$ : output voltage

Fig. 3.4 Equivalent circuits for (a) Mode 1, (b) Mode 2, (c) Modes 3 and 5, and (d) Mode 4.

Fig. 3.5 Output voltage results of PPT igniter circuits if an energy of $0.43 \mathrm{~mJ}$ is transferred. .37

Fig. 3.6 Block diagram of control circuit. 41

Fig. 3.7 Experimental setup of flyback circuit.

Fig. 3.8 Experimental waveforms of the flyback circuit. $v_{\text {Gate: }}$ gating signal of MOSFET,

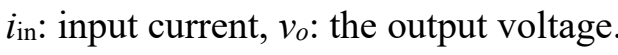

Fig. 3.9 (a) Maximum output voltage, and (b) maximum repetitive frequency versus charging current limit $I_{\text {lim. }}$.

Fig. 3.10 Efficiency of the flyback circuit versus the maximum output voltage.........45

Fig. 3.11 Energy distribution when the flyback circuit outputs $4.5 \mathrm{kV}$. .45 
Fig. 3.12 PPT integration test in vacuum environment. 46

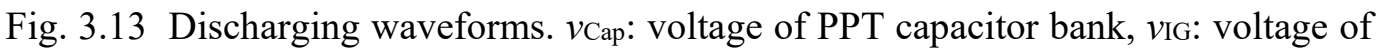
PPT igniter. 47

Fig. 3.14 Plasma generation in vacuum. 47

Fig. 4.1 Proposed quasi-resonant DC-DC converter using a single switch .50

Fig. 4.2 Model of a step-up transformer. .51

Fig. 4.3 Cockcroft-Walton voltage multiplier (VM). 52

Fig. 4.4 Steps to simplify the AC equivalent circuit: (a) complete equivalent circuit with $\Pi$-type circuit ( $\left.C_{\mathrm{in}}, L_{\mathrm{lk}}, C_{w}\right)$, (b) equivalent circuit with T-type circuit $\left(L_{1}, C_{T}, L_{2}\right)$, and (c) approximated equivalent circuit. .54

Fig. 4.5 Theoretical waveforms in steady state when the switch is turned off (a) between $t_{1}$ and $t_{2}$, and (b) at time $t_{1} . v_{\mathrm{GS}}$ : MOSFET gating signal, $i_{p}$ : primary side current, $v_{T}$ : lumped primary side voltage .55

Fig. 4.6 Equivalent circuits for (a) Mode 1, (b) Mode 2, and (c) Mode 3 if the MOSFET is turned off at time $t_{1}$. .57

Fig. 4.7 Flow chart to calculate $t_{2}$ numerically. 61

Fig. 4.8 Normalized voltage gain $v_{n}$ with respect to (a) $L_{m}=600 \mu \mathrm{H}, L_{1 \mathrm{k}}=0.6 \mu \mathrm{H}, R_{L}=$ $100 \mathrm{M} \Omega$; (b) $C_{T}=264.3 \mathrm{nF}, L_{\mathrm{lk}}=0.6 \mu \mathrm{H}, R_{L}=100 \mathrm{M} \Omega$.

Fig. 4.9 Root-mean-square of $i_{p}$ with (a) $L_{m}=600 \mu \mathrm{H}, L_{\mathrm{lk}}=0.6 \mu \mathrm{H}, R_{L}=100 \mathrm{M} \Omega$; (b) $C_{T}=264.3 \mathrm{nF}, L_{\mathrm{lk}}=0.6 \mu \mathrm{H}, R_{L}=100 \mathrm{M} \Omega$.

Fig. 4.10 Comparisons on output voltage between simulation and calculation results. Load is (a) $100 \mathrm{M} \Omega$, and (b) $18 \mathrm{M} \Omega$ 65

Fig. 4.11 Comparisons on $t_{1} / T$ between simulation and calculation results. Load is (a) $100 \mathrm{M} \Omega$, and (b) $18 \mathrm{M} \Omega$.

Fig. 4.12 (a) Prototype circuit board. (b) Experimental test setup. 68

Fig. 4.13 Waveforms when the MOSFET is turned off at time $t_{1}$. $v_{\mathrm{GS}}$ : MOSFET gating signal, $i_{p}$ : primary side current, $v T$ : lumped primary side voltage, $V_{o}$ : output voltage..69 Fig. 4.14 Waveforms when the MOSFET is turned off at time $t_{1}$. $v_{\mathrm{GS}}$ : MOSFET gating signal, $i_{p}$ : primary side current, $v$ Ds: drain-source voltage of MOSFET.

Fig. 4.15 Waveforms when the MOSFET is turned off at time $t_{2}$. $v_{\mathrm{GS}}$ : MOSFET gating signal, $i_{p}$ : primary side current, $v_{T}$ : lumped primary side voltage, $V_{o}$ : output voltage.. 71 Fig. 4.16 Waveforms when the MOSFET is turned off at time $t_{2}$. $v_{\mathrm{GS}}$ : MOSFET gating signal, $i_{p}$ : primary side current, $v_{\mathrm{DS}}$ : drain-source voltage of MOSFET. .71 
Fig. 4.17 Output voltage $V_{o}$ versus switching frequency $f_{s}$ by simulation and experiment. 72

Fig. 4.18 Full load of the proposed topology versus switching frequency. .73

Fig. 4.19 Experimental waveforms with a load of $12 \mathrm{M} \Omega$. $v_{\mathrm{GS}}$ : MOSFET gating signal, $i_{p}$ : primary side current, $v_{T}$ : lumped primary side voltage, $V_{o}$ : output voltage. 74

Fig. 4.20 (a) PPT system integration, and (b) plasma generation in vacuum chamber.

Fig. 4.21 Experimental waveforms in vacuum environment. $v$ firing: firing control, $v_{\text {igniter: }}$ voltage of PPT igniter, $v_{\text {Cap: }}$ voltage of PPT capacitor bank. 75

Fig. 4.22 Comparison on normalized voltage gain $v_{n}$ .78

Fig. 4.23 Comparison on MOSFET current between LLC resonant converter and proposed topology: (a) maximum current; (b) root-mean-square (RMS) current .80

Fig. 4.24 Comparison on (a) efficiency, and (b) switch conduction loss between LLC resonant converter and proposed topology. 80

Fig. 5.1 Quasi-resonant flyback converter for capacitor charging. 87

Fig. 5.2 Operating waveforms in the $k^{\text {th }}$ charging cycle of (a) VVS; (b) ZVS. vate: gating signal of MOSFET, $i_{L m}$ : current of $L_{m}, i_{\text {in: }}$ input current, $i_{p}$ : current of primary side, $i_{s}$ : current of secondary side, $v_{\mathrm{DS}}$ : drain-source voltage of MOSFET, $v_{o}$ : output voltage .88

Fig. 5.3 Equivalent circuit for (a) Mode 1, (b) Modes 2 and 4, and (c) Mode 3.......... 90 Fig. 5.4 Control circuit for (a) Ilim detection, (b) zero-voltage transition detection, (c) output voltage $V_{o}$ monitor, and (d) valley-voltage transition detection. 99

Fig. 5.5 The logic control of valley-voltage-switching (VVS)................................ 100

Fig. 5.6 Software flowchart of switching control for capacitor charging.................. 101

Fig. 5.7 Prototype circuit of quasi-resonant flyback converter. 102

Fig. 5.8 Experimental waveforms of the first two charging cycles when $I_{\mathrm{lim}}=3.8 \mathrm{~A}, C_{r}$ $=200.56 \mathrm{nF}$, and $C_{o}=1 \mu \mathrm{F} . v_{\text {Gate: }}$ gating signal, $i_{\text {in: }}$ input current, $v_{\mathrm{DS}}$ : voltage across MOSFET, $v_{0}$ : output voltage. 103

Fig. 5.9 Experimental waveforms of the first two charging cycles when $I_{\lim }=3.8 \mathrm{~A}, C_{r}$

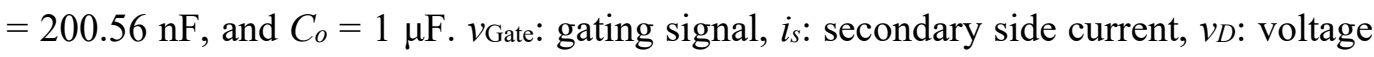
stress on diode $D_{s}$ 104

Fig. 5.10 Simulation waveforms of the first two charging cycles (a) without and (b) with circuit DC resistors when $I_{\lim }=3.8 \mathrm{~A}, C_{r}=200.56 \mathrm{nF}$, and $C_{o}=1 \mu \mathrm{F}$. $v_{\text {Gate: }}$ gating

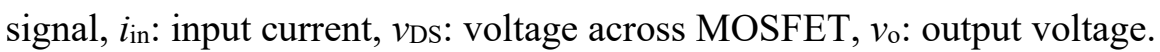
107 
Fig. 5.11 Simulation waveforms when $I_{\mathrm{lim}}=3.8 \mathrm{~A}, C_{r}=200.56 \mathrm{nF}, C_{o}=1 \mu \mathrm{F}$ with circuit DC resistors. $v_{\text {Gate: }}$ gating signal, $i_{\text {in: }}$ input current, $v_{\mathrm{DS}}$ : voltage across MOSFET, $v_{0}$ : output voltage. 108

Fig. 5.12 Capacitor charging waveforms when $I_{\mathrm{lim}}=3.8 \mathrm{~A}, C_{r}=200.56 \mathrm{nF}$, and $C_{o}=1$

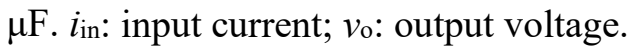
108

Fig. 5.13 Minimum resonant capacitance $C_{r}$,ZVS-min versus the capacitance $C_{o}$ if ZVS is obtained throughout the capacitor charging process. 109

Fig. 5.14 Charging current limit versus resonant capacitance when the load capacitance $C_{o}$ is $1 \mu \mathrm{F}$. 110

Fig. 5.15 Maximum output voltage $V_{o, \max }$ versus charging current limit $I_{\mathrm{lim}}$. 111

Fig. 5.16 Charging time and efficiency using ZVS with a fixed charging current limit $I_{\text {lim }}$ when load capacitance $C_{o}$ is $1 \mu \mathrm{F}$. 113

Fig. 5.17 Experimental waveforms of first two charging cycles when $I_{\mathrm{lim}}=1.6 \mathrm{~A}, C_{r}=$

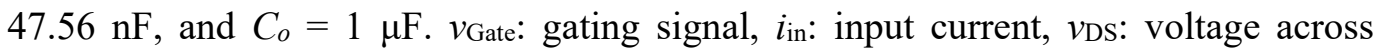
MOSFET, $v_{0}$ : output voltage. 114

Fig. 5.18 Experimental waveforms of first seven charging cycles when $I_{\text {lim-Vvs }}=1.6$ A,

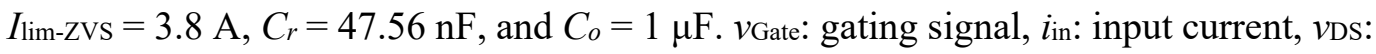
voltage across MOSFET, $v_{0}$ : output voltage. 115

Fig. 5.19 Experimental waveforms of first seven charging cycles when $I_{\text {lim-Vvs }}=1.6$ A, $I_{\mathrm{lim}-\mathrm{ZVS}}=3.8 \mathrm{~A}, C_{r}=47.56 \mathrm{nF}$, and $C_{o}=1 \mu \mathrm{F} . v_{\text {Gate: }}$ gating signal, $i_{s}$ : secondary side current, $v_{D}$ : voltage stress on diode $D_{s}$. 116

Fig. 5.20 Output voltage $v_{o}$ when $I_{\text {lim-VVS }}=1.6 \mathrm{~A}, I_{\mathrm{lim}-\mathrm{ZVS}}=3.8 \mathrm{~A}, C_{r}=47.56 \mathrm{nF}$, and $C_{o}=1 \mu \mathrm{F}$ 116

Fig. 5.21 Charging time and efficiency using hybrid switching when load capacitance $C_{o}$ is $1 \mu \mathrm{F}$. 118

Fig. 5.22 Comparisons with a conventional flyback converter on charging time and efficiency.

Fig. 5.23 Energy distribution of quasi-resonant flyback converter and conventional flyback converter when the load capacitors are charged to $600 \mathrm{~V}$. 122

Fig. A1 Two-port circuit network: (a) П-type, and (b) T-type. 129 


\section{List of Tables}

Table 1.1 Altitude decreasing rate with different initial orbit altitude ........................ 1

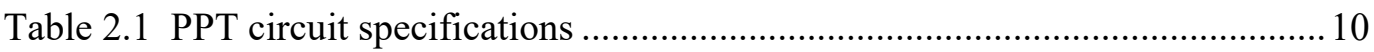

Table 2.2 DC characteristics of existing resonant converters ....................................20

Table 3.1 Failure in time of components in PPT igniter circuit ................................. 37

Table 3.2 Comparisons with other PPT igniter circuit .............................................. 38

Table 3.3 Components used in flyback circuit ......................................................... 40

Table 4.1 Parameter relationships between complete and approximated resonant tanks

Table 4.2 Components in simulation and prototype …............................................6 64

Table 4.3 Comparisons of proposed topology with existing resonant converters ....... 77 Table 4.4 Comparisons between flyback circuit and single switch quasi-resonant

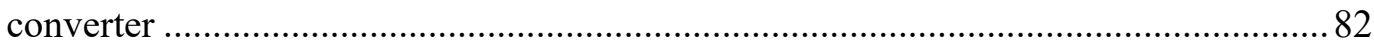

Table 5.1 Components used in the quasi-resonant flyback converter ......................... 98

Table 5.2 Parameters for studies on capacitor charging with ZVS.......................... 111

Table 5.3 Parameters for studies on capacitor charging with hybrid switching ........ 117 


\begin{abstract}
In nano-satellites, the pulsed plasma thruster (PPT) is a suitable electric propulsion system for orbit maintenance or attitude control due to its advantages of low mass (hundreds of grams), low power consumption (tens of Watts) and medium impulse bit (tens of $\mu \mathrm{Ns}$ ). The operating concept of PPT requires an igniter circuit to trigger the plasma generation, and an energy storage capacitor to ionize the propellant into plasma.

In this thesis, a flyback circuit is first proposed for a PPT igniter. When compared with other topologies, the proposed circuit utilizes parasitic capacitances instead of physical high voltage capacitor. Consequently, the reliability is improved by $13.4 \%$ since capacitors are fragile in power electronic systems. With a reduced number of components, the proposed circuit suits well to the size constraint of a nano-satellite. However, the flyback circuit uses a hard-switching technique. By its nature, it has the shortcomings of switching noise and switching loss.

The second topic of this thesis is to investigate an alternative soft-switching technique. This leads to a new quasi-resonant converter proposed for a PPT igniter. It consists of a resonant tank, a step-up transformer and a Cockcroft-Walton voltage multiplier. Inherent from the parallel resonant tank and series-parallel resonant tank, the proposed resonant tank has a boost capability, and the output voltage can be regulated at light loads. Unlike other resonant tanks, the proposed resonant tank can be driven by a single power switch. Thus, the switching control can be realized without a dead-time. In the quasi-resonant operation, the series resonant inductor operates in the discontinuous-current-mode. Hence, the switch can be turned on and off with zerocurrent to realize zero-current-switching (ZCS). This reduces switching noise, switching loss and voltage spikes. Compared to the resonant topologies using a switch bridge, the proposed quasi-resonant converter can realize ZCS at a switching frequency higher than
\end{abstract}


half of the resonant frequency. This leads to a better voltage boost capability. For example, the voltage gain of a parallel resonant converter is less than 1.33, while that of the proposed topology can be higher than 6.2. Due to a single switch implementation of and ZCS operation, the efficiency has been best improved by $12.05 \%$ as compared to an LLC resonant converter.

The third topic of this thesis investigates a quasi-resonant flyback converter for charging the capacitor in a PPT. By the quasi-resonant operation, the energy in the leakage inductance of transformer can be recycled to reduce energy loss. Moreover, the voltage spikes are relieved by the resonant capacitor when the switch is turned off. Depending on the operating conditions, the switch in quasi-resonant flyback converter is proposed to operate with zero-voltage-switching (ZVS) or hybrid switching throughout the capacitor charging process. In the hybrid switching mode, valleyvoltage-switching is adopted in the initial charging cycles before zero-voltage turn-on is obtained, and ZVS is used in the remaining charging process. Due to the quasi-resonant operation, both the switching control methods can reduce switching loss. However, hybrid switching is preferred in terms of efficiency because of shorter charging time and less conduction loss. Compared to a conventional flyback converter using a hardswitching technique, the capacitor charging efficiency has been best improved by $10.3 \%$. 


\section{List of Acronym}

$\mathrm{CCM}$

DCM

FHA

LCC-RC

LLC-RC

PPT

PRC

SPRC

SRC

$\mathrm{VM}$

VVS

$\mathrm{ZCS}$

ZCZV

ZVS
Continuous-current-mode

Discontinuous-current-mode

First harmonic approach

LCC resonant converter

LLC resonant converter

Pulsed plasma thruster

Parallel resonant converter

Series parallel resonant converter

Series resonant converter

Voltage multiplier

Valley-voltage-switching

Zero-current-switching

Zero-current zero-voltage

Zero-voltage-switching 


\section{Chapter 1}

\section{Introduction}

\section{$1.1 \quad$ Background}

In terms of weight, satellites are classified into big satellites $(>1000 \mathrm{~kg})$, small satellites $(500 \mathrm{~kg}-1000 \mathrm{~kg})$, mini-satellites $(100 \mathrm{~kg}-500 \mathrm{~kg})$, micro-satellites $(20 \mathrm{~kg}$ $100 \mathrm{~kg}$ ), and nano-satellites $(<20 \mathrm{~kg})$. As the technology development speeds up, nanosatellites are becoming more attractive due to their lower cost, shorter development time and good flexibility $[1,2]$.

Nano-satellites are usually launched into low earth orbit (LEO) with altitude between $160 \mathrm{~km}$ and $650 \mathrm{~km}$. In the lower altitude, satellites suffer from atmospheric drag, and will deorbit in a shorter time $[3,4]$. The satellite orbit disturbance caused by atmospheric drag has been studied in [4], and the altitude decreasing rate is shown in Table 1.1. Taking $150 \mathrm{~km}$ as the re-entry altitude, the estimated lifetime is 111 days if a satellite is located in the orbit altitude of $350 \mathrm{~km}$ without any propulsion system. To extend the lifetime of satellite, a propulsion system is required for orbit maintenance.

Table 1.1 Altitude decreasing rate with different initial orbit altitude

\begin{tabular}{ccc}
\hline \hline $\begin{array}{c}\text { Orbit altitude } \\
h(\mathrm{~km})\end{array}$ & $\begin{array}{c}\text { Nominal atmospheric density } \\
\rho_{0}\left(\mathrm{~kg} / \mathrm{m}^{3}\right)\end{array}$ & $\begin{array}{c}\text { Altitude decreasing rate } \\
\nabla \mathrm{a}(\mathrm{km} / \text { day })\end{array}$ \\
\hline 150 & $2.070 \times 10^{-9}$ & $174.013(\mathrm{crash})$ \\
180 & $5.464 \times 10^{-10}$ & 99.822 \\
200 & $2.789 \times 10^{-10}$ & 51.266 \\
250 & $7.248 \times 10^{-11}$ & 14.408 \\
300 & $2.418 \times 10^{-11}$ & 4.628 \\
350 & $9.518 \times 10^{-12}$ & 1.798 \\
\hline \hline
\end{tabular}


Besides orbit maintenance, a propulsion system can also be used for the satellite attitude control for sun tracking, nadir pointing mission, formation flying, etc. [5-7]. Hence, a propulsion is necessary for some space missions.

In general, there are two means of propulsion systems, i.e. chemical propulsion system and electric propulsion system. The chemical propulsion system can generate a high thruster force (hundreds of Newtons) [8]. This cannot be applied on small-scale satellites for precise control. Moreover, the large size and weight make it not applicable on nano-satellites.

The features of electric thruster systems have been discussed in $[9,10]$. Among different kinds of electric propulsion systems, the pulsed plasma thruster (PPT) has low power consumption (tens of Watts), medium impulse bit (tens of $\mu \mathrm{Ns}$ ) and compact size (less than $10 \mathrm{~cm} \times 10 \mathrm{~cm} \times 10 \mathrm{~cm}$ ). Hence, it is considered as a good candidate propulsion for nano-satellites which have power budget and size constraints.

As a promising electric propulsion, PPT has been demonstrated in many satellites after it was first used in spacecraft Zond 2 launched by the Soviet Union in 1964, such as Earth Observing 1 (US, launched in 2000) [11], Dawgstar (US, launched in 2003) [12], PROITERES (Japan, launched in 2012) [13], etc. To demonstrate the PPT operation in space for orbit maintenance and attitude control, the Satellite Research Centre (SaRC) in Nanyang Technological University (NTU) has developed a dual-axis PPT payload in satellite AOBA VELOX-III which was launched on $9^{\text {th }}$ December 2016.

The typical block diagram of a conventional PPT is shown in Fig. 1.1. The PPT system operates as follows $[5,14,15]$ :

- The capacitor charging circuit charges capacitor to a required energy. 
- The igniter circuit provides a high voltage (order of $\mathrm{kV}$ ) to the spark plugs, and an arc is generated. The arc releases free electrons to conduct the path between the electrodes.

- The energy in capacitor is released to ablate and ionize the Teflon propellant into plasma.

- The plasma is exhausted by the self-induced Lorentz force, and a thrust is generated.

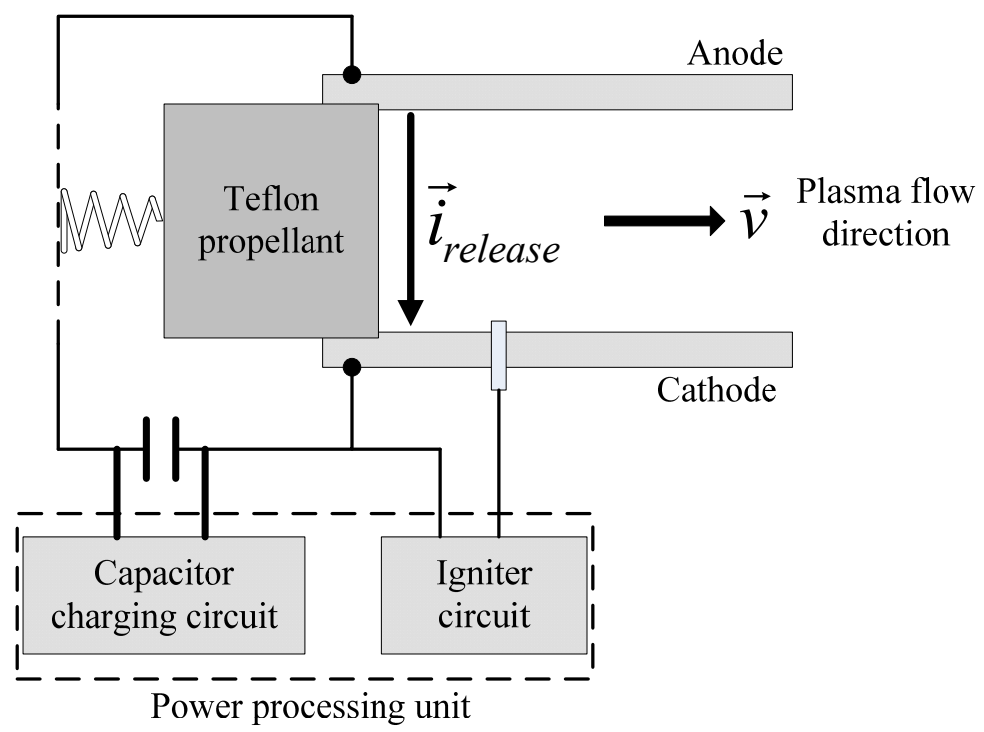

Fig. 1.1 Block diagram of a pulsed plasma thruster (PPT)

Various recent studies have been conducted to improve the PPT performance. To avoid depositions of carbon, liquid propellant [16] or residual atmosphere of the Earth in an altitude below $250 \mathrm{~km}$ [17] is used instead of Teflon propellant. In [18], the effect of an external magnetic field has been investigated. However, the igniter circuit and capacitor charging circuit are still required. 


\subsection{Motivations}

From the aforementioned operating principles, a PPT igniter circuit supplies a high voltage to the igniter spark plugs, and an arc is generated to conduct the path between electrodes. According to the Paschen's Law which gives the breakdown voltage, the required voltage depends on the vacuum level and gap between the igniter spark plugs. In general, it is an order of $\mathrm{kV}$. The challenge of PPT igniter circuit is the high voltage gain as the power bus of a nano-satellite normally provides a DC power supply of $5 \mathrm{~V}$. Besides the PPT igniter circuit, a capacitor charging circuit is also required. The charged voltage depends on the capacitance and energy needed by the satellite missions. Moreover, the charging time should satisfy the requirement of operating pulse frequency.

For satellite applications, the reliability is a big concern. From an industry-based survey in [19], power devices and capacitors are the two most fragile components in power electronic converters, contributing $30.7 \%$ and $18 \%$ to the circuit failure probability, respectively. Empirically, they are more probable to fail in the PPT circuits which output high voltage varying from hundreds of volts to order of kilo-volts. Besides, a nano-satellite has a volume constraint. Normally, the whole propulsion system is built in a compact size (less than $10 \mathrm{~cm} \times 10 \mathrm{~cm} \times 10 \mathrm{~cm}$ ). Lastly, the PPT itself has a low efficiency (less than 7\%) [20].

In the reported PPT studies, the igniter circuit employs a voltage-lift circuit in the first stage. Then, a high voltage is generated when the lifted voltage is transferred to the latter stages of circuit such as a ring tank [21]. However, the extra voltage-lift circuit challenges the volume of nano-satellites. For the capacitor charging circuit, a conventional flyback converter is commonly used due to the reduced number of components [12]. However, the hard-switching technique deteriorates the reliability of semiconductor switch and power efficiency. 
As the PPT igniter can operate with low power, this thesis investigates the feasibility of using transformer to store energy instead of high voltage capacitors. Without physical high voltage capacitors, the reliability can be improved. Another approach to improve the reliability is to introduce soft-switching techniques for PPT igniter and capacitor charging circuits. Compared to hard-switching, the voltage spikes can be mitigated. Moreover, the switching loss can be reduced. This benefits the efficiency of PPT electric circuits.

\subsection{Objectives}

The objectives of this thesis are as follows:

- Investigate the requirements of PPT circuits including the igniter circuit and capacitor charging circuit.

- Investigate the feasibility that a PPT igniter circuit uses parasitic capacitances of components. Without physical high voltage capacitors, the circuit reliability can be improved by avoiding the failure caused by them.

- Investigate a PPT igniter circuit using soft-switching technique to overcome the drawbacks of hard-switching such as switching loss, voltage spikes, etc. To reduce switch conduction loss, the implementation of a single switch is studied.

- Investigate the circuit behaviour throughout the capacitor charging process when a quasi-resonant flyback converter is used to charge the capacitor in a PPT. Through this research, an efficient switching control is studied. 


\subsection{Major Contributions}

The major contributions are as follows:

- Proposed and developed a flyback circuit for a PPT igniter. The circuit reliability is improved by using parasitic capacitances instead of high voltage capacitors.

In the proposed topology, the resonant voltage between the magnetizing inductance of transformer and parasitic capacitances can be used for a PPT igniter. When compared it to other topologies, physical high voltage capacitors are not required. Hence, the reliability is improved. Moreover, the step-up transformer enhances the output voltage. Hence, an extra voltage-lift circuit is not required. The output voltage is regulated by adjusting the charging current. This results in a simple switching control.

Using a reduced number of components, the circuit can be built in a compact size. This is useful for nano-satellite applications where the volume has constraint.

- Proposed and developed a quasi-resonant DC-DC converter for a PPT igniter.

The topology uses a soft-switching technique, and implements a single switch. This benefits the efficiency and reliability.

A new resonant tank is proposed which incorporates the parameters of transformer including leakage inductance, magnetizing inductance and winding capacitance. As the non-ideality parameters of transformer participate in the quasi-resonant operation, the adverse effects such as voltage and current spikes can be eliminated. The soft-switching technique reduces the switching loss because the switch can be turned on with zerocurrent, and off with zero-current zero-voltage.

Since the shunt magnetizing inductance provides the energy flow path of shunt capacitors in the resonant tank, a single switch is capable to drive the proposed resonant tank. A single switch implementation makes the circuit more reliable, eases the 
switching control without a dead-time, and leads to a better efficiency. Moreover, the range of switching frequency of the proposed converter can be extended to higher than half of the resonant frequency. Hence, the boost capability is better than other resonant converters operating with zero-current-switching (ZCS).

- Developed a quasi-resonant flyback converter for a PPT capacitor charging circuit. By soft-switching, the switching loss is reduced, and the efficiency is improved.

Depending on operating conditions, two switching control methods can be used which include zero-voltage-switching (ZVS) and hybrid switching. For the hybrid switching control method, valley-voltage-switching (VVS) is introduced in the initial stage. Once zero-voltage transition is obtained, the mode is switched to ZVS in the remaining charging process. Both switching modes can reduce switching-on loss and current spikes. Because of the quasi-resonant operation, the switching-off loss is minimized without reflected voltage stress on the switch from the output voltage.

Furthermore, the energy in transformer leakage inductance can be recycled to the load capacitor. Hence, no snubber is required to mitigate the voltage spikes on the semiconductor switch. This reduces the electromagnetic interference (EMI), and improves circuit reliability. Moreover, the diode can be turned on with zero-voltage, and off with zero-current. Hence, the turn-on overshoot voltage and recovery loss are eliminated. 


\subsection{Organization of the Thesis}

The outline of this thesis is as follows:

Chapter 2 reviews topologies for the PPT igniter and capacitor charging. These topologies use either hard-switching or soft-switching techniques.

Chapter 3 presents the proposed flyback circuit with parasitic components for a PPT igniter. The operating modes and mathematical analysis are described to explain the operating principle. To validate the proposed concept, simulation and experiment with a developed prototype circuit have been conducted.

Chapter 4 investigates the proposed single switch quasi-resonant DC-DC converter for a PPT igniter. The AC equivalent circuit of proposed topology is first discussed. The operating modes are then described, and the theoretical analysis based on the approximated AC equivalent circuit is deduced. The simulation and experimental studies have validated the feasibility of proposed topology. Comparisons between the flyback circuit presented in Chapter 2 and other resonant converters have revealed the novelty of proposed topology.

Chapter 5 discusses a quasi-resonant flyback converter for capacitor charging. The operating modes and corresponding mathematical analysis are first presented, and then followed by the discussion on the ZVS mode and hybrid switching mode. The simulation and experimental studies have been conducted to show the performance of the two switching control methods. Comparisons with a conventional flyback converter have shown that the proposed quasi-resonant flyback converter has a better charging efficiency when using hybrid switching.

Finally, Chapter 6 concludes this thesis, and presents the future work. 


\section{Chapter 2}

\section{Literature Review}

As introduced in Chapter 1, a pulsed plasma thruster (PPT) requires an igniter circuit to trigger the plasma generation, and a capacitor charging circuit to ablate and ionize the Teflon propellant into plasma.

In the existing PPT studies, the igniter circuit has provided a high voltage varying from $500 \mathrm{~V}$ to $15 \mathrm{kV}$ [5, 12-14, 21-23]. However, the PPT igniter requires a low power, varying from $0.08 \mathrm{~mW}$ to $22.5 \mathrm{~mW}$ with a pulse frequency of $1 \mathrm{~Hz}$ [14, 20, 23-25]. Hence, the requirements for PPT igniter circuit can be summarized as follows:

- High voltage gain: The power bus of nano-satellites usually provides $5 \mathrm{~V}$. Hence, the circuit should have a boost capability of generating an output voltage of up to several $\mathrm{kV}$.

- According to the Paschen's Law, the required voltage is affected by the vacuum level. Hence, the output voltage can be adjustable to adapt to the possible variance of voltage and power in orbit.

- Low output power is required to enable the igniter operation.

Since the required power of igniter depends much on the PPT configuration, only the output voltage specification is listed in Table 2.1.

Besides the igniter circuit, a PPT needs a capacitor charging circuit. The mission requirements determine the amount of the storage energy for ionizing Teflon propellant. In existing PPT studies, the energy varies from $2.3 \mathrm{~J}$ to $13.5 \mathrm{~J}[5,14,26]$. The requirements for the capacitor charging circuit are summarized as follows: 
- To obtain a desired energy, the circuit is capable of generating a high voltage to reduce the volume of capacitor bank.

- The charging time can satisfy the requirement of pulse frequency.

- The output voltage can be adjustable.

Constrained by the power budget of nano-satellites, the discharged energy from the capacitor bank per pulse is smaller than $1.5 \mathrm{~J}$, and the pulse frequency is set to $1 \mathrm{~Hz}$.

From the study, the specifications of PPT circuits are summarized in Table 2.1.

Table 2.1 PPT circuit specifications

\begin{tabular}{lc}
\hline \hline Input voltage & $5 \mathrm{~V}$ \\
Output voltage of igniter circuit & $>1 \mathrm{kV}$ \\
Output energy of capacitor charging circuit & $<1.5 \mathrm{~J}$ \\
Pulse frequency & $1 \mathrm{~Hz}$ \\
\hline \hline
\end{tabular}

\subsection{Candidate Topologies for PPT Igniter}

This section reviews existing circuit topologies which can meet the high voltage requirement of a PPT igniter. They are classified into two categories by the switching approaches, i.e. hard-switching and soft-switching techniques.

\subsubsection{Topologies Using Hard-switching}

\section{A. Transformer-less Topologies}

One approach to obtain a high voltage gain is to use step-up DC-DC converters based on conventional topologies such as boost converter, buck-boost converter, etc. [27-31]. By analyzing the operating modes of each inductor, the voltage gain can be obtained using volt-second balance of inductors. Normally the increase in conduction duty cycle 
results in a higher voltage gain. Hence, the switching control can be simply realized by pulse-width-modulation (PWM). For the topologies in [27-30], the voltage gains vary from 11 to 99 if the conduction duty cycle is 0.9 . The voltage gain cannot be increased further when the conduction duty cycle approaches extremely to unity due to the nonideality of components. For a nano-satellite whose power bus provides a voltage of $5 \mathrm{~V}$, the voltage gain should be at least 200 according to the specifications in Table 2.1. Thereby, such topologies have insufficient voltage boost capability for a PPT igniter. The cascade approach can improve voltage gain further [31]. However, the power devices suffer heavier voltage or current stress.

An alternative method is to use switched capacitors. The Cockcroft-Walton voltage multiplier (VM) shown in Fig. 2.1(a) has been employed in [14, 15, 32]. The capacitors in VM are charged by an AC input voltage $v_{\text {ac. }}$ For an $m$-stage VM, its ideal maximum output voltage is $m V_{\text {pk-pk }}$ where $V_{\text {pk-pk }}$ is the peak-to-peak value of $v_{\text {ac. }}$ The Marx generator shown in Fig. 2.1(b) has been investigated in [33-35]. The capacitors in Marx generator are charged by a DC input voltage $V_{\mathrm{DC}}$. A high voltage is generated when the switches configure the capacitors in series. Ideally, the maximum output voltage of an $m$-stage Marx generator is $m V_{\mathrm{DC}}$. Consequently, the output voltage of VM and Marx generator can be adjusted by the number of stages. Moreover, these topologies are capable of generating high power, e.g. up to $3 \mathrm{~kW}$ reported in [34]. However, the output voltage drop increases with the increased number of stages in practice due to the load current [36]. Hence, the voltage gain has a constraint. Furthermore, a number of stages make it difficult to miniaturize.

To enhance the voltage gain, some topologies have been proposed by combining a step-up DC-DC converter and VM [37-40]. Hence, the voltage gain is determined by the conduction duty cycle and number of VM stages. 


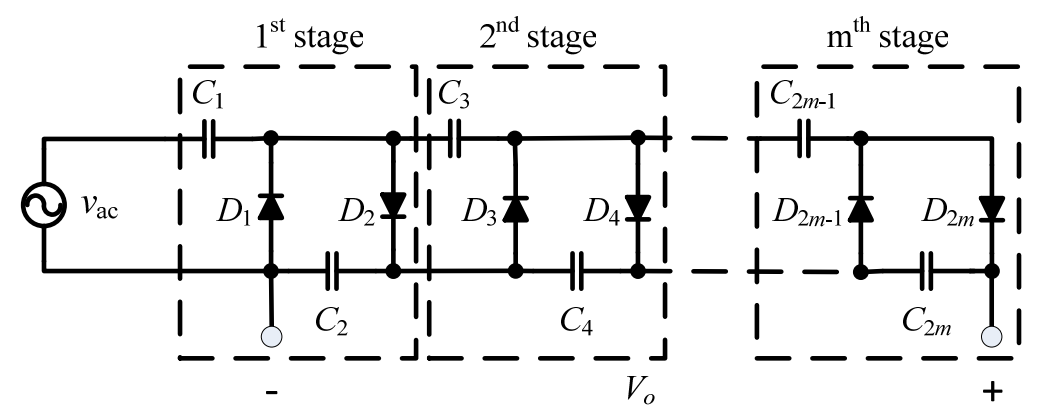

(a)

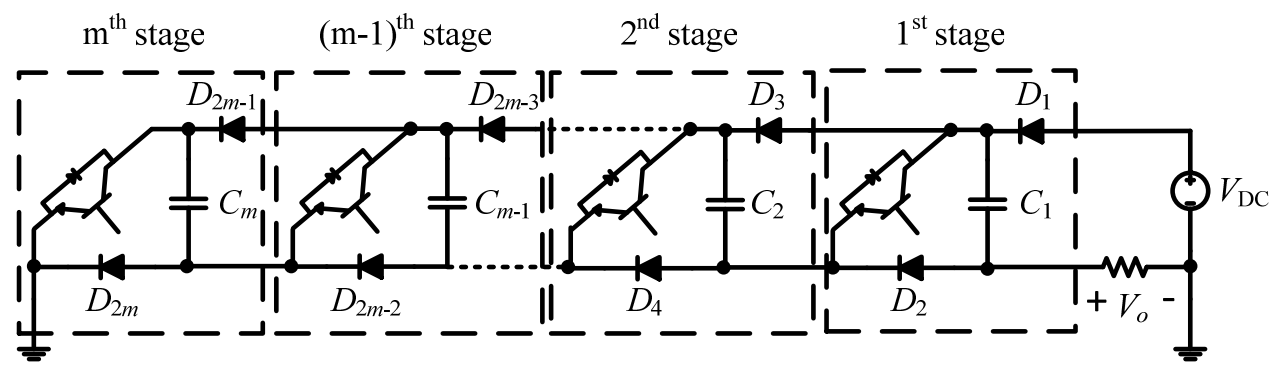

(b)

Fig. 2.1 Switched capacitors: (a) Cockcroft-Walton voltage multiplier (VM), and (b) Marx generator.

To reduce the number of components such as inductors in step-up DC-DC converters and diodes in switched capacitors, various topologies of pulsed power supply have been proposed for ozone generation, dielectric barrier discharge, etc. [41-44]. In [41], the topology employs a modified positive buck-boost converter. The energy is first stored in the inductor, and then transferred to a capacitor to generate a high voltage that is determined by the transferred energy and capacitance. In [42-44], a choke acts as a current source, and drives a resonant tank to form a high voltage. The quality factor of resonant tank determines the output voltage amplitude. Since these topologies do not use capacitor to store energy, the output power is limited. However, they are applicable for a PPT igniter as it just requires a small amount of power as aforementioned. Hence, they can be considered as an igniter circuit.

These transformer-less topologies can be developed and implemented easily. However, the terminals of high output voltage and low input voltage share the same 
circuit ground. This degrades the reliability of circuitry in the low voltage side. To ensure reliability and safety, a transformer is preferred as it can provide the galvanic isolation [45]. Moreover, a step-up transformer can perform a voltage-lift.

\section{B. Transformer-based Topologies}

Fig. 2.2 shows some circuit topologies which have been applied on a PPT igniter. In satellite Dragster [12], the PPT igniter circuit uses the topology shown in Fig. 2.2(a). A voltage of $1 \mathrm{kV}$ from a flyback circuit (not shown in the figure) is applied to a resonant tank, and the output voltage is doubled to $2 \mathrm{kV}$ for the PPT igniter. Satellite STSAT-2 has used the topology shown in Fig. 2.2(b) as the PPT igniter circuit. Capacitor $C_{1}$ needs to be pre-charged to a high voltage $(250 \mathrm{~V})$ by a DC-DC converter consisting of transformer $T_{1}$ and diode $D$ [21]. Then, the high output voltage is generated on the secondary side of step-up transformer when switch $S$ is turned on. In the PPT study in [46], a magnetic pulse compression circuit shown in Fig. 2.2(c) is used. The concept is similar to the application of resonant tank. By connecting stages of LC networks to form a magnetic pulse compression circuit, it can achieve a high quality factor to generate a high voltage.

While the circuitry in Fig. 2.2 is simple to build, an extra voltage-lift circuit is needed to pre-charge a capacitor. This increases the circuit complexity. 


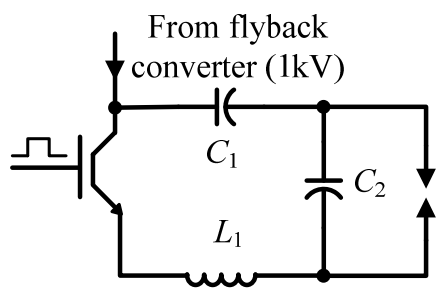

(a)

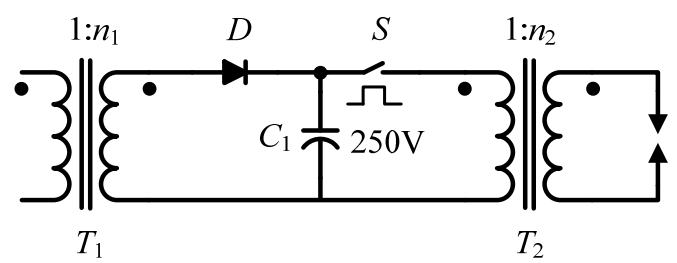

(b)

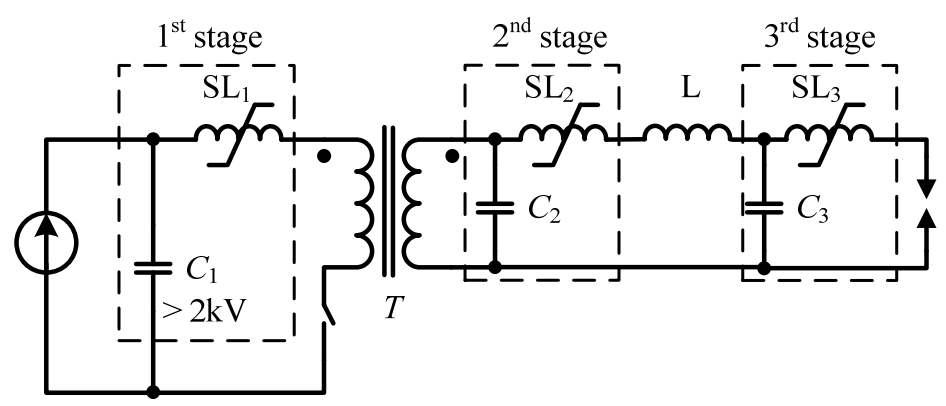

(c)

Fig. 2.2 PPT igniter circuit used in (a) satellite Dawgstar (US) [12], (b) satellite STSAT-2 (South of Korea) [21], and (c) PPT study [46].

To remove the voltage-lift circuit, a flyback converter has been used. A pulse voltage can be generated when the energy in transformer magnetizing inductance is transferred to the load capacitor. To obtain the desired high voltage, a modular flyback converter shown in Fig. 2.3 has been studied which configures basic modules in parallel or series [47]. However, a number of modules result in larger volume, higher cost, etc.

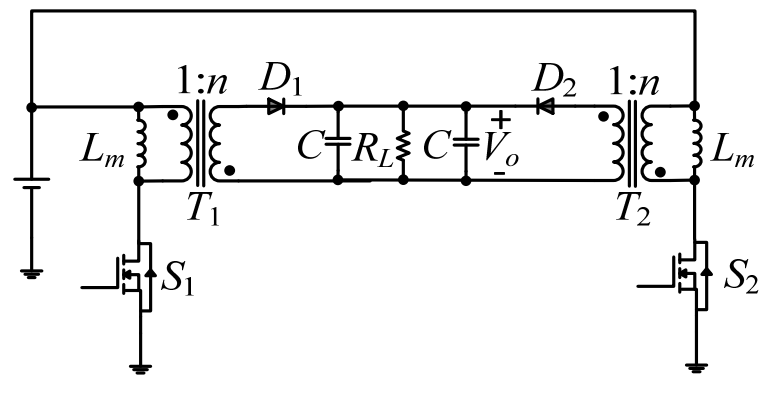

(a)

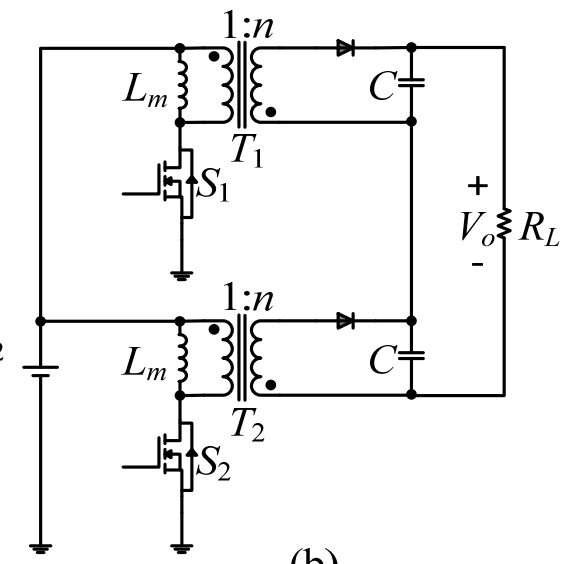

(b)

Fig. 2.3 Modular flyback converter with (a) parallel connection, and (b) series connection. 
All the aforementioned topologies in this section use a hard-switching technique. While they are able to provide the desired high voltage, the hard-switching approach leads to voltage and current spikes, switching noise and switching loss due to the nonideality of transformer. This reduces the reliability of semiconductor switches, which is a big concern for satellite applications. Moreover, the circuit efficiency is deteriorated greatly. This shortcoming can be addressed by a soft-switching technique, and will be discussed in the following sections.

\subsubsection{Topologies Using Soft-switching}

To overcome the drawbacks caused by hard-switching, a soft-switching technique is preferred since zero-current-switching (ZCS) and/or zero-voltage-switching (ZVS) can be realized due to the resonant operation.

The soft-switching technique has been investigated in topologies by adding an LC resonant circuit to the conventional boost converter [31, 48, 49]. With a reduced switching loss, the efficiency can be improved. However, a transformer is preferred due to its nature of galvanic isolation as aforementioned. This section focuses on the most well investigated transformer-based resonant converters which have the following advantages:

- The non-ideality parameters of transformer can be incorporated into the basic circuit operation. Hence, the adverse effects such as voltage and current spikes can be eliminated. This is helpful for the component reliability.

- The switching frequency can be high, varying from tens to hundreds of $\mathrm{kHz}$. This benefits the size reduction of magnetic components.

- Soft-switching reduces the switching loss to improve efficiency. The switching noise is reduced as well, reducing the risk of electromagnetic interference (EMI) issue. 
- The resonant converters may have boost capability, which is beneficial to the high voltage generation.

The block diagram of a transformer-based resonant converter is shown in Fig. 2.4. It consists of a switch bridge, a resonant tank, a step-up transformer and a rectifier. The resonant converters are classified by the types of resonant tanks.

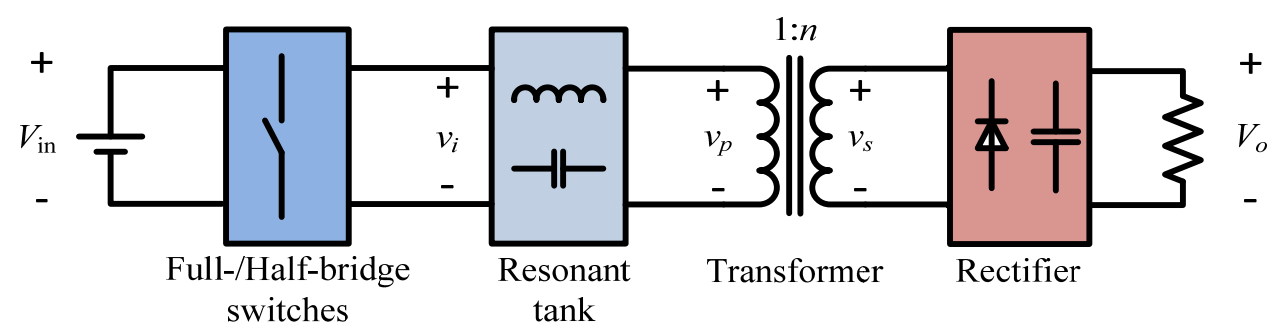

Fig. 2.4 Block diagram of a transformer-based resonant converter.

The most well investigated resonant converters are series resonant converter (SRC), parallel resonant converter (PRC), LCC resonant converter (LCC-RC) and LLC resonant converter (LLC-RC). LCC-RC and LLC-RC are grouped as hybrid series-parallel resonant converter (SPRC). Their respective resonant tanks are shown in Fig. 2.5. Among these resonant tanks, the parallel resonant tank and LLC resonant tank have been used in PPT studies $[50,51]$. Others have been investigated for various high-voltage applications such as X-ray, industrial processes, electrostatic precipitators, etc.

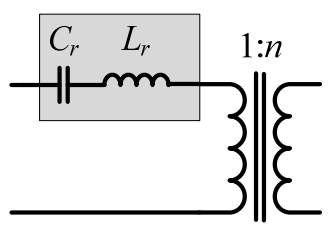

(a)

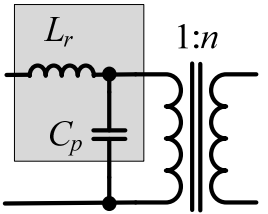

(b)

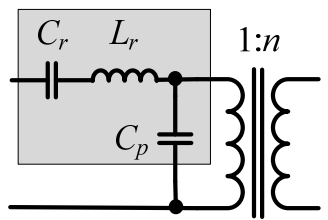

(c)

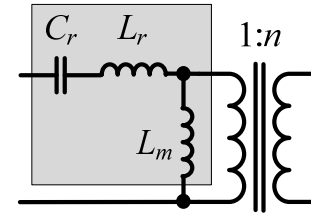

(d)

Fig. 2.5 Types of resonant tanks: (a) series resonant tank, (b) parallel resonant tank, (c) LCC resonant tank, and (d) LLC resonant tank. 
To discuss the characteristics of resonant tanks, the normalized switching frequency $f_{n}$ and normalized voltage gain $v_{n}$ are defined as

$$
\begin{gathered}
f_{n}=\frac{f_{s}}{f_{r}}, \\
v_{n}=\frac{V_{p, \mathrm{pk}-\mathrm{pk}}}{2 V_{\mathrm{in}}}
\end{gathered}
$$

where $f_{s}$ is the switching frequency; $f_{r}$ is the natural resonant frequency; $V_{p, \text { pk-pk }}$ is the peak-to-peak value of the transformer primary side voltage $v_{p}$; and $V_{\text {in }}$ is the input voltage. The normalized voltage gain $v_{n}$ can be used to study the DC characteristics of resonant converters irrespective of the rectifier type.

The first harmonic approach (FHA) is usually used to analyze the normalized voltage gain [52]. Using FHA, the AC equivalent circuit of a resonant converter can be modeled as Fig. 2.6. $v_{\mathrm{ac}}$ is the fundamental component generated by a switch bridge. $Z_{s}$ and $Z_{p}$ are the equivalent impedances of series and parallel resonant components, respectively. $R_{\mathrm{ac}}$ is the $\mathrm{AC}$ equivalent resistor reflected to the primary side of transformer from loads. By the principle of impedance divider, the normalized voltage gain $v_{n}$ is deduced by

$$
v_{n}=\frac{v_{p}}{v_{\mathrm{ac}}}=\frac{R_{\mathrm{ac}} / / Z_{p}}{Z_{s}+R_{\mathrm{ac}} / / Z_{p}} .
$$

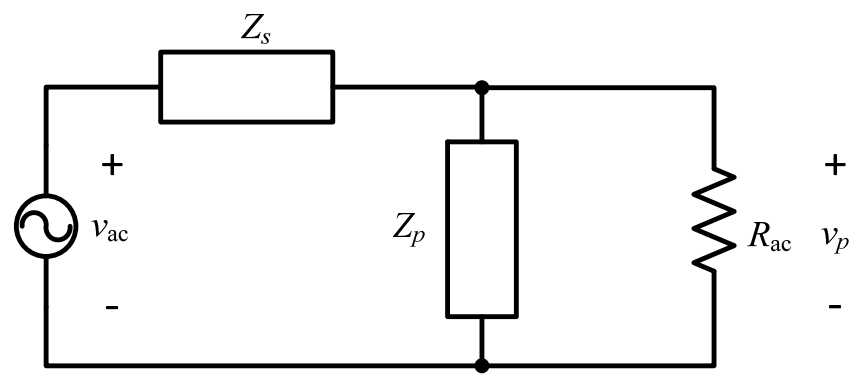

Fig. 2.6 Common AC model of a resonant converter for first harmonic approach (FHA). 


\section{A. Series Resonant Converter (SRC)}

The resonant tank of SRC is shown in Fig. 2.5(a). As summarized in [53], SRC can obtain zero-voltage turn-on with $f_{n}>1$, and zero-voltage turn-off with $0.5<f_{n} \leq 1$. However, SRC is preferable to operate with $f_{n} \leq 0.5$ when operating with discontinuouscurrent-mode (DCM), because ZCS for both turn-on and turn-off can be obtained [36, $54-56]$.

By FHA, the normalized voltage gain $v_{n}$,SRC of SRC is

$$
v_{n, \mathrm{SRC}}=\frac{1}{\sqrt{1+Q_{\mathrm{SRC}}^{2}\left(f_{n}-f_{n}^{-1}\right)^{2}}}
$$

where $Q_{\mathrm{SRC}}$ is the load quality factor of SRC, and determined by $Q_{\mathrm{SRC}}=\frac{1}{R_{\mathrm{ac}}} \sqrt{\frac{L_{r}}{C_{r}}}$.

From (2.4), the maximum normalized voltage gain is 1 occurring at the resonant frequency $\left(f_{n}=1\right)$. Hence, the series resonant tank does not contribute to the boost capability. Moreover, the output voltage regulation is poor under light load condition, i.e. $Q_{\mathrm{SRC}} \approx 0$. This makes it less favorable as the PPT igniter requires output voltage regulation at light loads.

\section{B. Parallel Resonant Converter (PRC)}

The parallel resonant tank of PRC is shown in Fig. 2.5(b). When $f_{n}$ is smaller than 0.5 , the PRC is operated with DCM and ZCS $[57,58]$. Similar to SRC, PRC is preferable to work with ZCS to reduce the switch conduction loss [59-61].

By FHA, the normalized voltage gain $v_{n, \text { PRC }}$ of PRC is

$$
v_{n, \mathrm{PRC}}=\frac{1}{\sqrt{\left(1-f_{n}^{2}\right)^{2}+\left(f_{n} / Q_{\mathrm{PRC}}\right)^{2}}}
$$

where $Q_{\mathrm{PRC}}$ is the load quality factor of PRC, and determined by $Q_{\mathrm{PRC}}=R_{\mathrm{ac}} \sqrt{\frac{C_{r}}{L_{r}}}$. 
When compared to SRC, PRC improves the voltage boost capability, and the output voltage regulation at light loads. Its circulating current is relatively independent of loads. This makes it suitable for low-output-voltage high-output-current applications in terms of efficiency [52]. Hence, it is not a suitable candidate for a PPT igniter which requires high voltage with low power.

\section{LCC Resonant Converter (LCC-RC)}

To overcome the drawback of PRC, LCC-RC has been investigated in [62-64] for high voltage power supply. Its resonant tank is shown in Fig. 2.5(c). LCC-RC can operate in three modes, i.e. below resonance in DCM with $f_{n} \leq 0.5$, below resonance in continuous-current-mode $(\mathrm{CCM})$ with $0.5<f_{n} \leq 1$, and above resonance in CCM with $f_{n}>1$ [62]. Considering the boost capability and efficiency, LCC-RC is preferred to operate with DCM to obtain ZCS.

By FHA, the normalized voltage gain $v_{n, \mathrm{LCC}}$ of LCC-RC is

$$
v_{n, \mathrm{LCC}}=\frac{1}{\sqrt{\left[1+n_{c}^{-1}-n_{c} f_{n}^{2}\right]^{2}+\left[Q_{\mathrm{LCC}}\left(f_{n}-f_{n}^{-1}\right)\right]^{2}}}
$$

where $Q_{\mathrm{LCC}}$ is the load quality factor of LCC-RC, and $n_{c}=C_{p} / C_{r} \cdot Q_{\mathrm{LCC}}$ is determined by $Q_{\mathrm{LCC}}=\frac{1}{R_{\mathrm{ac}}} \sqrt{\frac{L_{r}}{C_{r}}}$.

\section{LLC Resonant Converter (LLC-RC)}

LLC resonant converter (LLC-RC) is another solution to overcome the drawback of PRC by adding one more resonant inductor $L_{m}$ [65-69]. Its resonant tank is shown in Fig. 2.5(d). LLC-RC operation consists of three modes, i.e. ZVS with $f_{n}>1$, ZVS with $\frac{1}{\sqrt{m}}<f_{n} \leq 1$, and ZCS with $f_{n} \leq \frac{1}{\sqrt{m}} . m$ is defined as $\frac{L_{m}+L_{r}}{L_{r}}$. LLC-RC does not have boost capability when $f_{n}$ is higher than 1 [69]. In contrast, LLC-RC exhibits the peak 
voltage gain when operating in ZVS region with $\frac{1}{\sqrt{m}}<f_{n} \leq 1$. To take advantage of LLC-RC boost capability, literatures have investigated the ZVS mode when LLC-RC is operated with $\frac{1}{\sqrt{m}}<f_{n} \leq 1$

By FHA, the normalized voltage gain $v_{n, \mathrm{LLC}}$ which is determined by

$$
v_{n, \mathrm{LLC}}=\frac{1}{\sqrt{\left(\frac{m}{m-1}-\frac{f_{n}^{-2}}{(m-1)}\right)^{2}+Q_{\mathrm{LLC}}^{2}\left(f_{n}-f_{n}^{-1}\right)^{2}}}
$$

where $Q_{\mathrm{LLC}}$ is the load quality factor of LLC-RC, and $m=\left(L_{m}+L_{r}\right) / L_{r} \cdot Q_{\mathrm{LLC}}$ is determined by $Q_{\mathrm{LLC}}=\frac{1}{R_{\mathrm{ac}}} \sqrt{\frac{L_{r}}{C_{r}}}$.

\section{E. Characteristics of Existing Resonant Converters}

The DC characteristics of the most well investigated resonant converters are summarized and listed in Table 2.2.

Table 2.2 DC characteristics of existing resonant converters

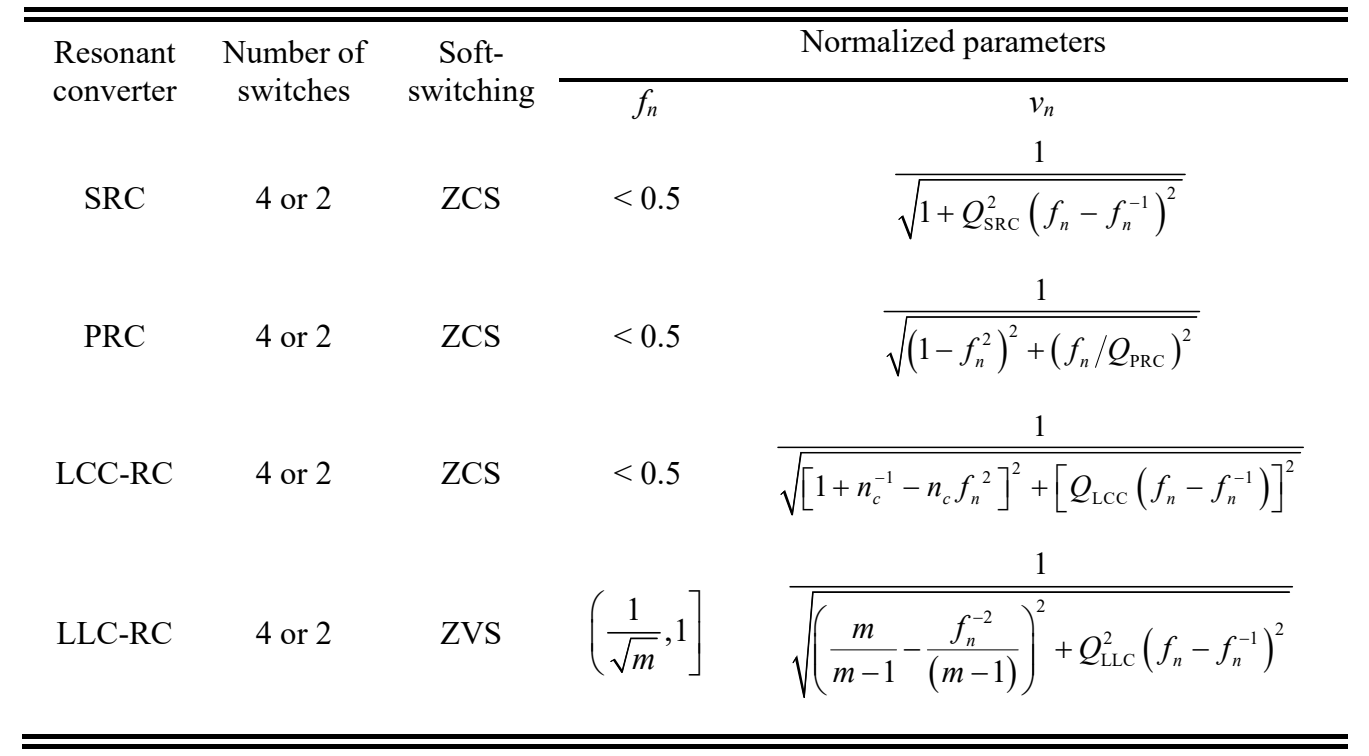


For the resonant tanks shown in Fig. 2.5, the energy flow path of resonant capacitors should be provided by one leg of switches. Hence, these resonant tanks should be driven by a switch bridge to obtain the resonant operation. This means a switch bridge formed by two or four switches is required.

As reviewed in the earlier sections, ZVS and ZCS can be obtained under different ranges of switching frequency. The switch conduction loss in DCM is typically the smallest due to ZCS at turn-on and turn-off. Hence, SRC, PRC and LCC-RC are operated with ZCS and $f_{n} \leq 0.5$.

Some authors have derived more accurate expressions for the normalized voltage gains of resonant converters $[70,71]$. However, such expressions are complex because they depend on the load conditions. For simplification, the normalized voltage gains listed in Table 2.2 are deduced by FHA.

\section{F. Other Topologies}

Some authors have proposed phase-shifted converters based on full-bridge switches for plasma display panel, renewable energy sources, ozone generator, etc. [72-74]. Such topologies can absorb the leakage and magnetizing inductances of transformer. Similar to LLC-RC, all the four switches can obtain ZVS to reduce switching loss. However, it is difficult for lagging leg switches to achieve ZVS at light loads [75]. Hence, phaseshifted converters are widely used for high power applications.

To realize ZVS at light loads, the dual-active-bridge converter with phase-shifted control has been proposed [76]. However, the switches in high voltage side suffers much voltage stress. This makes it unsuitable for high-voltage applications such as PPT igniter.

The quasi-resonant flyback converters reviewed in Section 2.2.3 can generate high voltage using a single switch [77-79]. To improve the efficiency at light loads, they are operated with valley-voltage-switching (VVS), and the switching frequency is lowered 
by selecting a proper valley-voltage to turn on the power switch. The switching-on loss and inrush current exist. Hence, quasi-resonant flyback converters working with VVS are unsuitable for low-power applications such as PPT igniter.

\subsection{Candidate Topologies for Capacitor Charging}

A PPT system requires a capacitor charging circuit to store energy for Teflon propellant ionization. Theoretically, the non-isolated step-up DC-DC converters reviewed in Section 2.1 can be used to charge capacitor. For example, a conventional boost DC-DC converter has been studied to charge a battery in [80, 81$]$. However, this thesis prefers utilizing of a transformer to take advantage of its galvanic isolation. Hence, this section focuses on the transformer-based topologies suitable for the capacitor charging.

\subsubsection{Flyback and Forward Converters}

A conventional flyback converter shown in Fig. 2.7(a) has been commonly used in existing PPT studies due to its low number of components $[5,12,21,22]$. It behaves as a current source when operating with continuous-current-mode (CCM) [82]. This shortens the charging time, and minimizes the conduction loss. To fully demagnetize and avoid the saturation of transformer, the flyback converter has been proposed to operate with discontinuous-current-mode (DCM) [83, 84]. The output voltage is regulated by the conduction duty cycle and turns ratio of transformer. However, the voltage gain cannot be increased further when the conduction duty cycle approaches extremely to unity due to the non-ideality of components.

As investigated in [85], a charge pump is added to the traditional flyback converter, and the voltage boost capability is doubled. For a higher output voltage, one approach is 
to combine a flyback transformer and a voltage multiplier (VM) [86]. Another approach is to configure a number of basic flyback converters in series [87].

The forward converter shown in Fig. 2.7(b) has also been discussed for battery charging $[88,89]$. When it is compared to flyback converter, the components in a forward converter dissipate less energy for the same output power [90]. However, the forward converter is derived from a buck DC-DC converter. Hence, it has a limited voltage boost capability [90].

To take advantages of flyback and forward converters, the flyback-forward topology has been investigated [91]. The two converters are connected in series, and share the same transformer. Using this topology, the voltage gain and efficiency are improved.

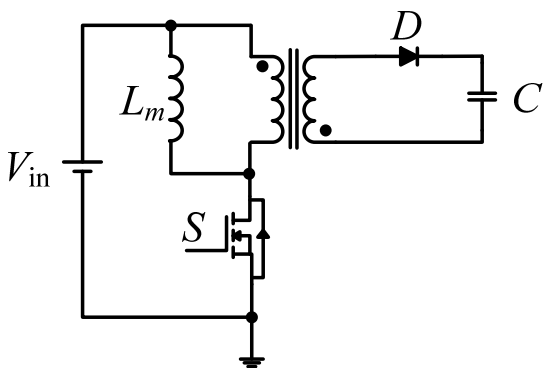

(a)

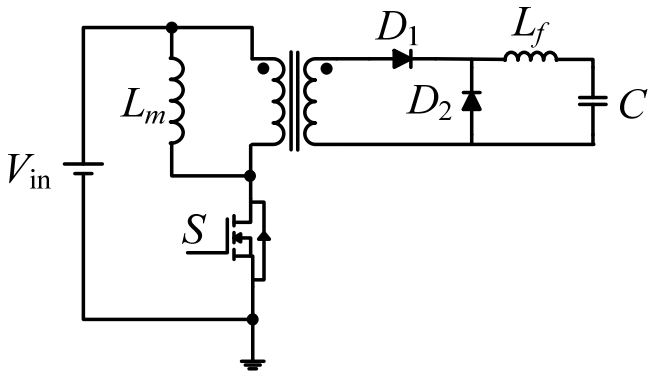

(b)

Fig. 2.7 (a) Conventional flyback converter, and (b) forward converter.

The aforementioned flyback, forward and flyback-forward converters have the advantages of simple switching control and compact volume. However, the use of hardswitching technique leads to voltage spikes on the semiconductor switch due to the leakage inductance of transformer. This degrades circuit reliability, and it is a critical concern for satellite applications. Hence, a snubber circuit is required to suppress the voltage spikes which results in energy loss [92-94]. 


\subsubsection{Resonant Converters}

To overcome the drawbacks of hard-switching, a soft-switching technique can be introduced. The converters in Fig. 2.5 have been employed for capacitor or battery charging [51, 69, 95-97]. Besides, other types of resonant converters have also been investigated such as L3C2 resonant converter [98], LCL resonant [99], etc.

For the PPT applications, the capacitor operation varies widely throughout the charging process. It behaves like a short circuit initially, and an almost open circuit in the end [100]. As a result, the soft-switching cannot be guaranteed during the entire charging process. Take PRC as an example whose resonant tank is shown in Fig. 2.5(b). The resonance cannot be formed when the load capacitor behaves like a short circuit in the beginning. For the SRC whose resonant tank is shown in Fig. 2.5(a), the circulating current is load-independent. Hence, the soft-switching control can be implemented during the charging process $[95,97]$. However, SRC has no voltage boost capability. For LCC-RC and LLC-RC, their corresponding resonant tanks have a series resonant capacitor and a series resonant inductor as shown in Figs. 2.5(c) and (d), respectively. They behave as SRC when the load capacitor behaves like a short circuit. Hence, LCC$\mathrm{RC}$ and LLC-RC can obtain resonant operation. As reviewed in Section 2.1.2, they exhibit voltage boost capability. Consequently, they have been investigated for vehicle battery charging $[69,101]$.

The operating modes of LCC-RC and LLC-RC are usually analyzed in steady state $[71,102]$. If they are used to charge capacitor from zero to a desired voltage, the analysis and switching implementation become more complex. Moreover, these resonant topologies require a switch bridge as presented in Section 2.1.2. Using multiple switches reduces circuit reliability, and a dead-time is required to avoid the short circuit of same leg. 


\subsubsection{Quasi-resonant Converters}

To inherit the advantages of conventional flyback, forward and resonant converters, the quasi-resonant converters have been widely investigated [103-111]. The quasiresonance between the magnetizing inductance of transformer and resonant capacitance makes that soft-switching can be obtained to reduce switching loss and switching noise.

The quasi-resonant forward converter can be realized by an auxiliary circuit which uses a small forward-type auxiliary transformer [103]. This topology requires two transformers which increase the circuit design complexity. To implement only a single transformer, multiple switches have been introduced to achieve zero-voltage-switching (ZVS) $[104,105]$. However, the use of multiple switches makes the switching control complex, and degrades circuit reliability.

In $[106,107]$, a voltage doubler is added to the secondary side of forward transformer. One capacitor in the voltage doubler acts as the resonant capacitor. While the circuit complexity is reduced, the forward converter has a limited voltage gain as it is derived from a buck converter.

To improve the voltage gain, quasi-resonant flyback converters have been proposed. In [108], the quasi-resonant operation can be realized by replacing the diode in the secondary side with a semiconductor switch. As surveyed in [19], semiconductor switch has a higher failure rate than diode. Hence, the reliability is reduced. For the quasiresonant flyback converters in [109-111], a resonant capacitor is added to the conventional flyback converter.

In the reported literatures, the quasi-resonant flyback converter uses valley-voltageswitching (VVS) whoes key waveforms are shown in Fig. 2.8 [77-79, 112]. As the switch is turned on at valley-voltage, the switching-on loss is minimized. To improve the efficiency at light loads, the switching frequency can be reduced by selecting a 
proper valley-voltage to turn on the switch. For example, the load power is smaller when the switch is turned on at the third valley-voltage. Fig. 2.8(a) shows the VVS scheme discussed in [77-79]. The output voltage is limited to smaller than $n V_{\text {in }}$ ( $n$ is the transformer turns ratio of the secondary winding to the primary winding; $V_{\text {in }}$ is the input voltage). Fig. 2.8(b) illustrates the VVS scheme discussed in [112]. The output voltage is enhanced to higher than $n V$ in. However, the energy loss will be caused by the freewheeling diode in parallel with the power switch. This deteriorates the circuit efficiency.

In the reported literatures, only the operations in steady state have been discussed. As for the repetitive charging and discharging in PPT applications, further study on the charging process needs be conducted to achieve a better charging efficiency.

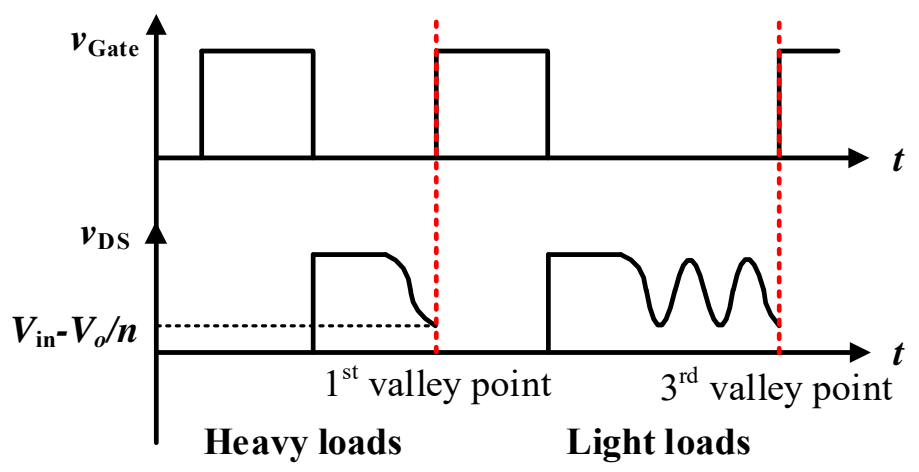

(a)

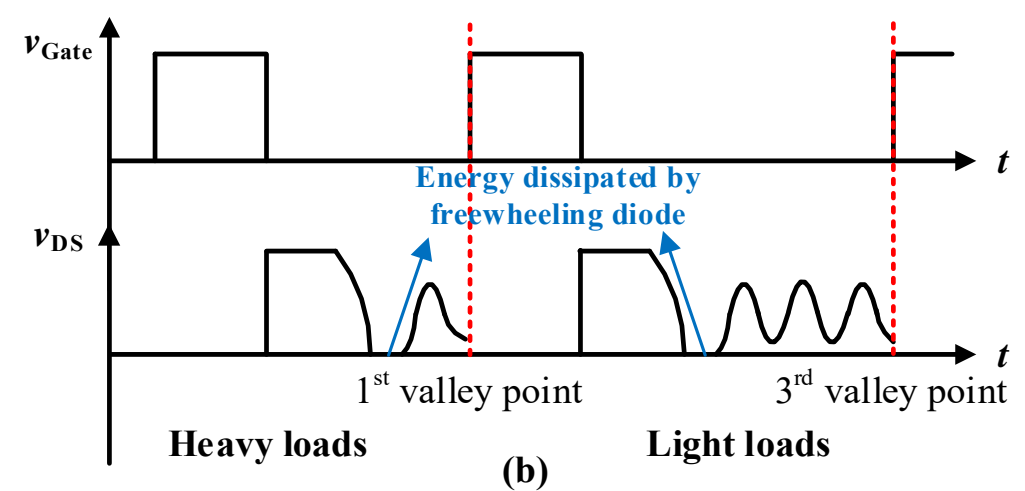

Fig. 2.8 Key waveforms of quasi-resonant flyback converter using valley-voltage-switching (VVS) when (a) $V_{o}<n V_{\text {in }}$, and (b) $V_{o}>n V_{\text {in. }}$. 


\subsection{Summary}

In this chapter, the requirements of PPT igniter circuit and capacitor charging circuit have been concluded from the existing PPT studies. Based on the study, the circuit topologies that can be applied in a PPT have been reviewed.

For a PPT igniter circuit, topologies using a hard-switching technique have the advantages such as compact size, simple switching control, etc. The biggest issue is that the hard-switching technique results in voltage and current spikes, which degrades the reliability of semiconductor switches. To overcome the drawbacks, a soft-switching technique is introduced. The most well investigated resonant converters have been reviewed. Among these resonant converters, SRC, PRC and LCC-RC are preferred to operate with ZCS while LLC-RC is operated with ZVS considering the efficiency and voltage gain. The four power switches in phase-shifted converters can obtain ZVS in high power applications. This makes it less favorable for a PPT igniter circuit.

For the capacitor charging circuit, the flyback and forward converters are simple to develop. However, the hard-switching causes drawbacks such as switching loss, voltage spikes, etc. For the resonant converters in Fig. 2.5, soft-switching needs complex switching control throughout the charging process. The quasi-resonant converters have the advantages of conventional converters and soft-switching technique.

The next chapter will present a proposed flyback circuit with parasitic components for a PPT igniter using hard-switching. 


\section{Chapter 3}

\section{Pulse Voltage Generation Using a Flyback Circuit with Parasitic Components}

\subsection{Introduction}

As reviewed in Chapter 2, the topologies for high-voltage low-power applications can be built in a compact size using a hard-switching technique. Due to the form factor constraint in nano-satellites, they are the promising solutions to the pulsed plasma thruster (PPT) igniter circuit.

From the reviewed existing topologies, it is observed that they share the same circuit structure shown in Fig. 3.1, including an energy storage unit, a high voltage forming unit, and a pulse load. The high pulse voltage is generated when the energy in the storage unit is transferred to the high voltage forming unit. The energy storage unit can be an inductor [41-44], or a capacitor $[12,21,26]$. The high voltage forming unit can use a capacitor $[41,47]$, a transformer [21], or a resonant tank [12, 42-44].

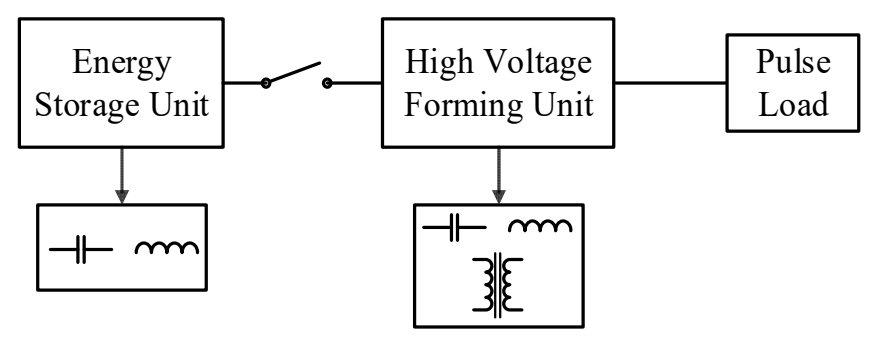

Fig. 3.1 Block diagram of a pulse power supply. 
In these topologies, high voltage capacitors are required. Even though the dielectric stability of capacitor has been investigated widely, the high voltage capacitor remains a fragile component [113]. As surveyed by [19], the capacitor contributes $18 \%$ to the failure of power electronic system. Hence, the capacitor is the vulnerable component which affects the circuit reliability, especially for high voltage pulse power applications.

Considering the reliability and safety, a transformer is introduced to provide the galvanic isolation. It is a fact that the transformer and semiconductor components have parasitic capacitances. The resonance occurs between the magnetizing inductance of transformer and parasitic capacitances when the power switch is turned off. It can be used for high pulse voltage generation. Thereby, the physical high voltage capacitors can be removed to improve reliability. In this chapter, a flyback circuit with parasitic components is investigated for a PPT igniter.

\subsection{Flyback Circuit with Parasitic Components}

The proposed topology for a PPT igniter is shown in Fig. 3.2. A metal-oxidesemiconductor field-effect transistor (MOSFET) with freewheeling diode $D$ is employed as semiconductor switch $S$. The spark plugs in Fig. 3.2(a) are two metal tips connected to the output terminals of the igniter circuit. In Fig. 3.2(b), $C_{w}, C_{\mathrm{oss}}, C_{s}$ and $C_{\mathrm{ig}}$ are the winding capacitance of transformer, output capacitance of MOSFET, parasitic capacitance of diode $D_{s}$ and capacitance formed by the spark plugs, respectively. 


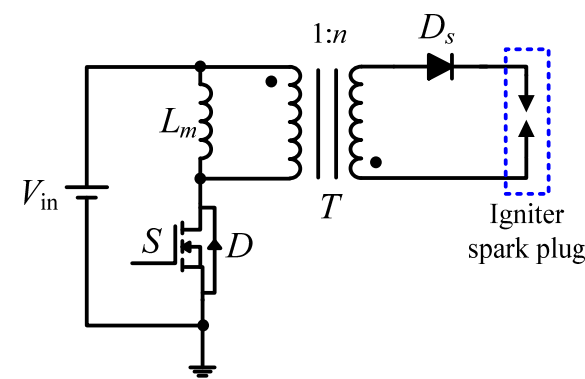

(a)

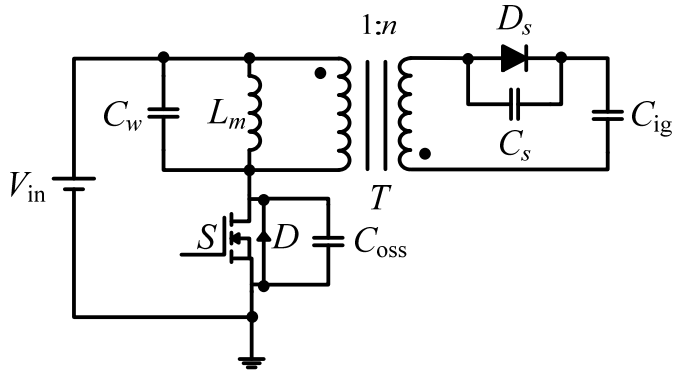

(b)

Fig. 3.2 Flyback circuit (a) without, and (b) with parasitic capacitances.

When the MOSFET is turned on, energy starts to store in the parasitic capacitances $\left(C_{w}, C_{\text {oss }}, C_{s}, C_{\mathrm{ig}}\right)$ and transformer magnetizing inductance $L_{m}$. The major energy is stored in the magnetizing inductance which functions as the energy storage unit. When the switch is turned off, the resonance occurs between parasitic capacitances and transformer magnetizing inductance, and a high pulse output voltage is yielded. In this approach, physical high voltage capacitors are removed to improve the reliability. With a reduced number of components, the circuit can be built compactly. The output voltage can be adjusted by the charging current limit. Since the flyback circuit without high voltage physical capacitor is proposed for low-power application, the remaining resonant energy can make the current of transformer reverse after the pulse voltage is generated. This would reset the flux in the magnetic core to prevent saturation, and a reset clamp circuit is not required [90, 93].

\subsection{Operating Principle}

In this analysis, the leakage inductance of transformer is neglected to simplify the theoretical analysis. To study the potential maximum voltage that the topology is capable to generate, the spark plugs of PPT igniter is modeled by capacitor $C_{\mathrm{ig}}$ given that an arc 
is not generated. The nominal waveforms are shown in Fig. 3.3. The operation is subdivided into five modes.

1) Mode $1\left[t_{0}-t_{1}\right]$ : Power switch $S$ (MOSFET) is turned on at time $t_{0}$. The transformer primary side is applied by the input voltage $V_{\mathrm{in}}$, and the current $i_{L}$ of magnetizing inductance increases linearly. The input current $i_{\text {in }}$ equals to $i_{L}$. The corresponding equivalent circuit is shown in Fig. 3.4(a). If the initial current of magnetizing inductance is zero, the primary side voltage $v_{p}$ and the current $i_{L}$ of magnetizing inductance are

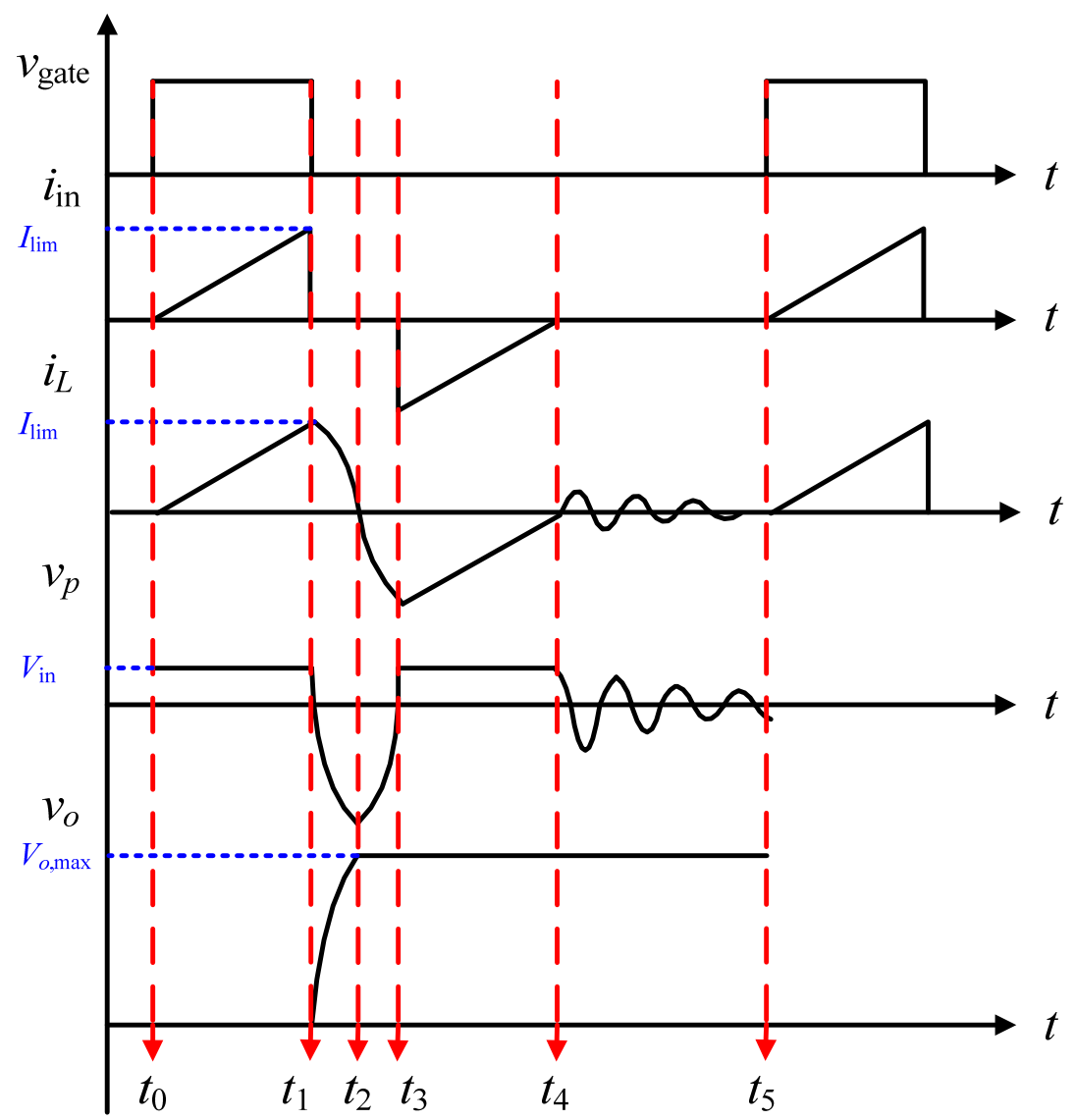

Fig. 3.3 Nominal waveforms of flyback circuit. $v_{\text {Gate }}$ : MOSFET gating signal, $i_{\text {in }}$ input current, $i_{L}$ : current of magnetizing inductance, $v_{p}$ : primary side voltage, $v_{o}$ : output voltage. 


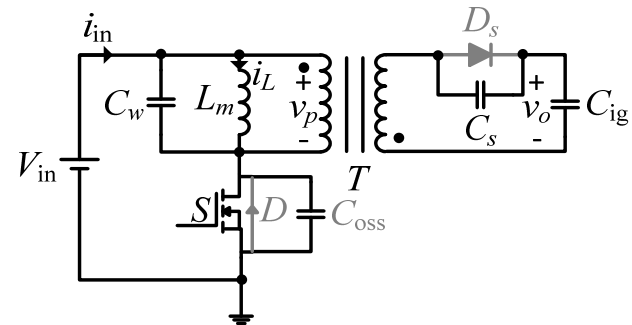

(a)

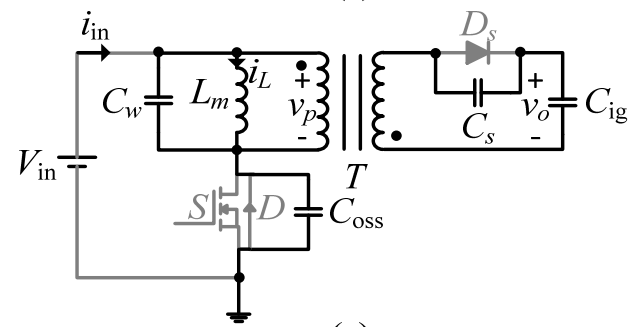

(c)

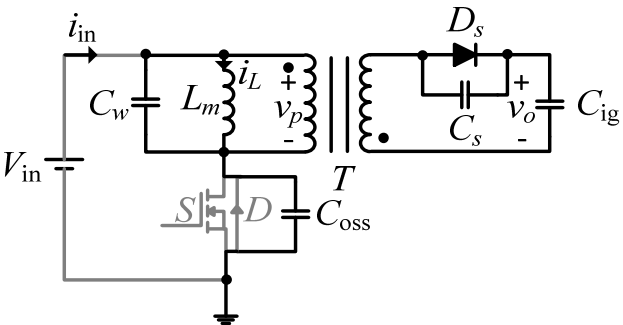

(b)

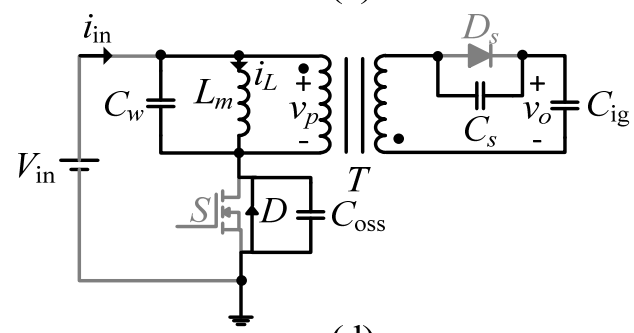

(d)

Fig. 3.4 Equivalent circuits for (a) Mode 1, (b) Mode 2, (c) Modes 3 and 5, and (d) Mode 4.

$$
\left\{\begin{array}{l}
v_{p}(t)=V_{\text {in }} \\
i_{L}(t)=\frac{V_{\text {in }}}{L_{m}}\left(t-t_{0}\right)
\end{array} .\right.
$$

The MOSFET is turned off at time $t_{1}$ when the current $i_{L}$ reaches the current limit $I_{\text {lim. }}$. Hence, the time duration $T_{1}$ of Mode 1 is

$$
T_{1}=\frac{L_{m} I_{\lim }}{V_{\text {in }}} .
$$

2) Mode $2\left[t_{1}-t_{2}\right]$ : Once the MOSFET is turned off, diode $D_{s}$ in the secondary side starts to conduct. Freewheeling diode $D$ is blocked. Fig. 3.4(b) shows the equivalent circuit of Mode 2. The resonance occurs among $L_{m}$ and capacitances $\left(C_{w}, C_{\text {oss }}, C_{s}\right.$ and $C_{\mathrm{ig}}$. These parasitic capacitances are lumped into a capacitance $C_{r}$ by

$$
C_{r}=C_{w}+C_{\mathrm{oss}}+n^{2} \frac{C_{s} C_{\mathrm{ig}}}{C_{s}+C_{\mathrm{ig}}}
$$

where notation $n$ is the transformer turns ratio of the secondary winding to the primary winding. 
The resonant behavior can be described by the following second-order differential equation:

$$
\frac{d^{2} i_{L}}{d t^{2}}+\omega_{o}^{2} i_{L}=0
$$

where $\omega_{o}=\frac{1}{\sqrt{L_{m} C_{r}}}$.

The initial conditions of Mode 2 are $i_{L}\left(t_{1}\right)=I_{\text {lim }}$ and $v_{p}\left(t_{1}\right)=V_{\text {in }}$. From (3.4), the solutions to $i_{L}$ and $v_{p}$ are

$$
\left\{\begin{array}{l}
i_{L}(t)=I_{\text {lim }} \cos \left(\omega_{o}\left(t-t_{1}\right)\right)+\frac{V_{\text {in }}}{Z_{r}} \sin \left(\omega_{o}\left(t-t_{1}\right)\right) \\
v_{p}(t)=V_{\text {in }} \cos \left(\omega_{o}\left(t-t_{1}\right)\right)-Z_{r} I_{\lim } \sin \left(\omega_{o}\left(t-t_{1}\right)\right)
\end{array}\right.
$$

where $Z_{r}=\sqrt{L_{m} / C_{r}}$.

From (3.5), the maximum amplitude of primary side voltage is $V_{p \text {,max }}$ given by

$$
V_{p, \text { max }}=\sqrt{V_{\text {in }}^{2}+\frac{L_{m}}{C_{r}} I_{\text {lim }}^{2}} .
$$

Hence, the maximum output voltage $V_{o \text {, max }}$ and maximum voltage stress on the MOSFET $V_{\mathrm{DS}, \max }$ are

$$
\begin{gathered}
V_{o, \text { max }}=n V_{p, \text { max }}, \\
V_{\mathrm{DS}, \text { max }}=V_{\text {in }}+V_{p, \text { max }} .
\end{gathered}
$$

From (3.6) and (3.7), the output voltage can be adjusted by the current limit $I_{\text {lim }}$ to adapt to different voltage requirements. The generated high voltage can be used for a PPT igniter.

In Mode 2, the input current $i_{\text {in }}$ remains zero. This mode ends when $i_{L}$ drops to zero at time $t_{2}$. Hence, the time duration can be determined by solving the equation $i_{L}(t)=0$. 
3) Mode $3\left[t_{2}-t_{3}\right]$ : Due to the resonance, the current $i_{L}$ reverses, and the amplitude of $v_{p}$ drops. Diode $D_{s}$ in the secondary side is blocked. The corresponding equivalent circuit is shown in Fig. 3.4(c). As freewheeling diode $D$ is still blocked, the input current $i$ in remains zero in this mode.

4) Mode $4\left[t_{3}-t_{4}\right]$ : When $v_{p}=V_{\text {in }}$ at time $t_{3}$, freewheeling diode $D$ is conducting. Hence, the input current $i_{\text {in }}$ equals to current $i_{L}$, and the input voltage $V_{\text {in }}$ is applied to the primary side again. The equivalent circuit of Mode 4 is shown in Fig. 3.4(d). As the current $i_{\text {in }}$ reverses, the flux in magnetic core is reset. Hence, the ferrite core saturation can be prevented, and a reset clamp circuit is not required.

5) Mode $5\left[t_{4}-t_{5}\right]$ : When $v_{p}<V_{\text {in }}$ after time $t_{4}$, the freewheeling diode is blocked. As the parasitic capacitances have been charged to $V_{\text {in }}$ in Mode 4, there is a slight oscillation between the transformer magnetizing inductance and parasitic capacitances. In practice, the oscillation will terminate shortly due to the damping by the DC resistor of the circuit. The equivalent circuit is the same as Mode 3 shown in Fig. 3.4(c).

\subsection{Benchmarking with Other PPT Igniter Circuits}

Table 3.2 summarizes the benchmarking of proposed flyback circuit with other PPT igniter circuits.

\subsubsection{Design Complexity}

The circuit topologies shown in Fig. 2.2 require an extra voltage-lift circuit. This increases the circuit complexity. For the modular flyback converter shown in Fig. 2.3, multiple transformers are used. This challenges the miniaturization. Since the flyback circuit utilizes the least number of components, it can be built easily and compactly. 


\subsubsection{Number of High Voltage Capacitors}

The PPT igniter circuit in Fig. 2.2(a) uses a resonant tank as the high voltage forming unit. Two high voltage capacitors are introduced. The PPT igniter circuit in Fig. 2.2(b) uses a high voltage capacitor as the energy storage unit. For an $m$-stage magnetic pulse compression circuit in Fig. 2.2(c) and an $m$-module flyback converter shown in Fig. 2.3, $m$ high voltage capacitors are required to yield high voltage.

Instead of a physical high voltage capacitor, the flyback circuit in this chapter uses parasitic capacitances to generate a high pulse voltage. Hence, a physical high voltage capacitor is removed.

\subsubsection{Power Level}

As summarized in Table 3.2, the power delivered by the circuits in Fig. 2.2 are determined by the energy storage capacitor in the voltage-lift circuit and the lifted

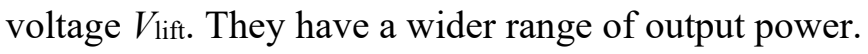

In contrast, the circuits discussed in Chapter 3 and [47] use the magnetizing inductance of transformer to store energy. The power level is constrained by the selected ferrite core. For example, a ferrite core with a longer effective magnetic path length or a larger saturation magnetic flux density is able to generate a higher power level. For the custom transformer in Table 3.3 , the calculated saturation current is $7.186 \mathrm{~A}$, and the maximum output power is $2.085 \mathrm{~mW}$ if the pulse frequency is $1 \mathrm{~Hz}$.

\subsubsection{Maximum Output Voltage}

To compare the voltage boost capability, several premises are defined. First, circuits use the same parameters of step-up transformer in Table 3.3. Second, the capacitance in the energy storage unit and high voltage forming unit is the same as the parasitic 
capacitance in flyback circuit, i.e. $2.08 \mathrm{nF}$ from Table 3.3. Third, $C_{2}$ is much larger than $C_{1}$ in Fig. 2.2(a).

Based on the premises, the lifted voltage $\mathrm{V}_{\text {lift }}$ in Fig. 2.2 is $643 \mathrm{~V}$ if an energy of 0.43 $\mathrm{mJ}$ is transferred. The current limit $I_{\text {lim }}$ of flyback circuit and 1-module flyback converter is $3.27 \mathrm{~A}$, while that of a 4-module flyback converter is $1.635 \mathrm{~A}$. The output voltages are shown in Fig. 3.5. It is observed that the modular flyback converter in [47] generates the lowest output voltage since the turns ratio of transformer does not help enhance the voltage boost capability. The PPT igniter circuit with a resonant tank in [12] also has a poor voltage boost capability. Benefitting from a step-up transformer and the stages of magnetic pulse compression circuit, the circuit in [46] generates the highest output voltage. For the PPT igniter circuit in [21] and the proposed flyback circuit using parasitic components, they have a comparable output voltage. Compared to other topologies, the proposed flyback circuit has a medium voltage boost capability.

\subsubsection{Reliability}

The circuit reliability depends on many factors, such as components, operating environment, system design, etc. As investigated in [113], the lifetime of ceramic capacitors is less affected by voltage compared to electrolytic capacitors and film capacitors. Moreover, ceramic capacitors have small size and large range of operating temperature. Hence, they are suitable to apply in a PPT of nano-satellites.

To simplify the discussion, all components are assumed to operate with safe margins, and their corresponding failure rates remain the same irrespective of operation conditions such as output voltage, power level, etc. Hence, the statistics in Table 3.1 extracted from [114] can be used to estimate the reliability improvement. From the listed failure rates of relevant components in an igniter circuit including ceramic capacitor, MOSFET, diode and transformer, the ceramic capacitor contributes $13.4 \%$ to the igniter 
circuit failure. Hence, the reliability can be improved a lot without a physical high voltage capacitor.

Table 3.1 Failure in time of components in PPT igniter circuit

\begin{tabular}{cc}
\hline \hline Type & Failure rate in a year \\
\hline Ceramic capacitor & $1 / 250$ \\
MOSFET & $1 / 1142$ \\
Diode & $1 / 50$ \\
Transformer & $1 / 200$ \\
\hline \hline
\end{tabular}

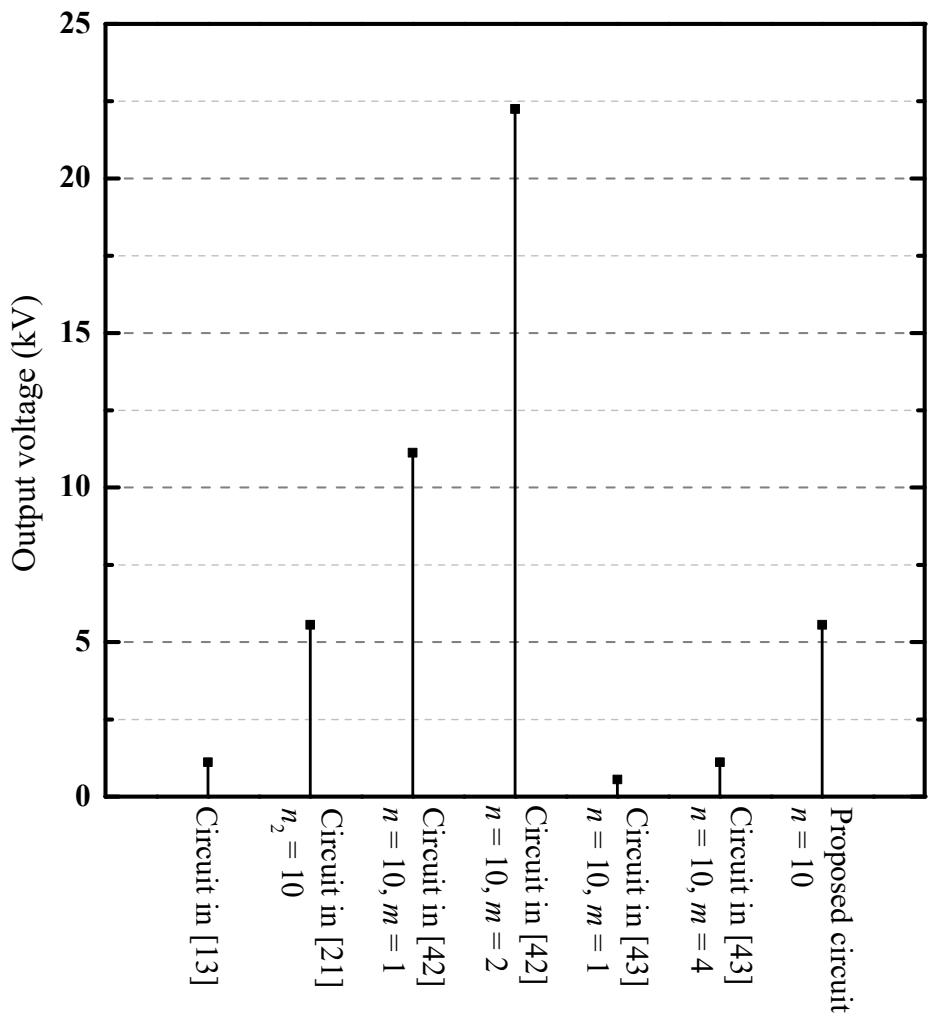

Fig. 3.5 Output voltage results of PPT igniter circuits if an energy of $0.43 \mathrm{~mJ}$ is transferred. 
Table 3.2 Comparisons with other PPT igniter circuit

\begin{tabular}{|c|c|c|c|c|}
\hline Topology & $\begin{array}{l}\text { Extra voltage- } \\
\text { lift circuit }\end{array}$ & $\begin{array}{l}\text { Number of high } \\
\text { voltage capacitor }\end{array}$ & $\begin{array}{l}\text { Power in energy } \\
\text { storage unit }\end{array}$ & $\begin{array}{l}\text { Maximum output } \\
\text { voltage }\end{array}$ \\
\hline Circuit using ring tank $[12]^{*}$ & $\sqrt{ }$ & 2 & $\frac{1}{2} \frac{C_{1} C_{2}}{C_{1}+C_{2}} V_{\text {lift }}^{2}$ & $2 V_{\text {lift }}$ \\
\hline Ignition circuit $[21]^{* *}$ & $\sqrt{ }$ & 1 & $\frac{1}{2} C_{1} V_{\text {lift }}^{2}$ & $n_{2} V_{\text {lift }}$ \\
\hline $\begin{array}{l}\text { Circuit using magnetic pulse } \\
\text { compression }[46]^{* * *}\end{array}$ & $\sqrt{ }$ & $m$ & $\frac{1}{2} C_{1} V_{\mathrm{lift}}^{2}$ & $n 2^{m} V_{\text {lift }}$ \\
\hline Modular flyback converter $[47]^{* * * *}$ & $x$ & $m$ & $\frac{1}{2} m L_{m} I_{\lim }^{2}$ & $m I_{\lim } \sqrt{\frac{L_{m}}{C}}$ \\
\hline Proposed flyback circuit & $x$ & 0 & $\frac{1}{2} L_{m} I_{\lim }^{2}$ & $n \sqrt{V_{\mathrm{in}}^{2}+\frac{L_{m}}{C_{r}} I_{\mathrm{lim}}^{2}}$ \\
\hline
\end{tabular}

* The topology is shown in Fig. 2.2(a). $V_{\text {lift }}$ is the lifted voltage on capacitors $C_{1}$ and $C_{2}$.

** The topology is shown in Fig. 2.2(b). $V_{\text {lift }}$ is the lifted charged voltage on capacitor $C_{1}$.

*** The topology is shown in Fig. 2.2(c). $m$ is the stage number of magnetic pulse compression circuits. $V_{\text {lift }}$ is the lifted charged voltage on capacitor $C_{o}$.

$* * * * \quad m$ is the number of basic flyback modules. $C$ is the capacitor in each basic module. 


\subsection{Simulation and Experimental Results}

\subsubsection{Prototype Development}

In this study, the drain-source breakdown voltage of MOSFET is a big concern for high voltage generation. Hence, a MOSFET STB28NM50N is selected whose drainsource breakdown voltage is $500 \mathrm{~V}$. According to the requirements of PPT igniter, the turns ratio $n$ should be larger than 4 to achieve an output voltage higher than $2 \mathrm{kV}$ from (3.6) - (3.8). To develop a prototype, a transformer with a turns ratio of 10 is custom made in laboratory. Considering the design margin to assure the safety of MOSFET, the output voltage is set to smaller than $4.5 \mathrm{kV}$.

From the study in [115], the winding capacitance of transformer converges a value $C t$ when the number of turns is larger than $10 . C t$ is determined by the diameter of selected copper wire, the gap between adjacent turns and the length of one turn. Constrained by the bobbin length, a single layer with 15-turn wire is coiled as the primary winding. Consequently, the secondary winding has 150 turns of wire. To reduce the voltage spikes on the MOSFET, the transformer leakage inductance $L_{\mathrm{lk}}$ is reduced by bifilar windings [116]. The parameters of custom made transformer are extracted by an LCR meter E4098A.

As the output voltage is regulated by charging current, the saturation current of ferrite core should be considered. In the prototype, a ferrite core B66423U0315K187 is selected, and the saturation current of the custom made transformer is $7.186 \mathrm{~A}$. According to the output characteristics of STB28NM50N under an applied voltage of 5 $\mathrm{V}$, its current rating is smaller than $7 \mathrm{~A}$. On the other hand, the output voltage is limited to $4.5 \mathrm{kV}$ which can be obtained by a current limit of $3.03 \mathrm{~A}$ theoretically. Hence, the ferrite core and MOSFET can meet the current requirement. 
Table 3.3 Components used in flyback circuit

\begin{tabular}{llc}
\hline \hline Transformer & Ferrite core & B66423U0315K187 \\
& Winding capacitance $C_{w}$ & $1.862 \mathrm{nF}$ \\
& Magnetizing inductance $L_{m}$ & $80.78 \mu \mathrm{H}$ \\
& Turns of primary winding $n_{p}$ & 15 \\
& Turns of secondary winding $n_{s}$ & 150 \\
& Leakage inductance $L_{\mathrm{lk}}$ & $106 \mathrm{nH}$ \\
& DC resistance $R_{\mathrm{dc}}$ & $19.5 \mathrm{~m} \Omega$ \\
\hline MOSFET & Part number & $\mathrm{STB} 28 \mathrm{NM} 50 \mathrm{~N}$ \\
& Drain-source breakdown voltage $V_{\mathrm{BD}}$ & $500 \mathrm{~V}$ \\
& Output capacitance $C_{\mathrm{oss}}$ & $418 \mathrm{pF}$ \\
& On resistance $R_{\mathrm{M} \text { osfET }}$ & $135 \mathrm{~m} \Omega$ \\
& Diode threshold voltage $R_{\mathrm{thr}}$ & $1.5 \mathrm{~V}$ \\
& Freewheeling diode resistance $R_{D}$ & $71.4 \mathrm{~m} \Omega$ \\
\hline Diode & Part number & $\mathrm{SF} 1600-\mathrm{TR}$ \\
& Parasitic capacitance $C_{s}$ & $40 \mathrm{pF}$ \\
& Forward voltage $V_{F}$ & $3.4 \mathrm{~V}$ \\
& On resistance $R_{\text {Diode }}$ & $3.4 \Omega$ \\
\hline \hline
\end{tabular}

The block diagram of control circuit is illustrated in Fig. 3.6. A 555-timer controls the operating frequency of PPT igniter. A monostable multivibrator forms a one-shot at the rising edge of timer signal. The one-shot sets the output of D-type flip flop to high voltage level, and the MOSFET is turned on. The transformer starts to charge. A sensing resistor of $50 \mathrm{~m} \Omega$ is employed to monitor the current. The current limit $I_{\text {lim }}$ is determined by the reference voltage $V_{\text {ref }}$ from a digital-to-analog converter (DAC). When the current reaches $I_{\lim }\left(I_{\lim }=V_{\text {ref }} / R_{\text {sensing }}\right)$, the comparator will output a rising edge. This makes the D-type flip flop output a low voltage level, and the MOSFET is turned off. 


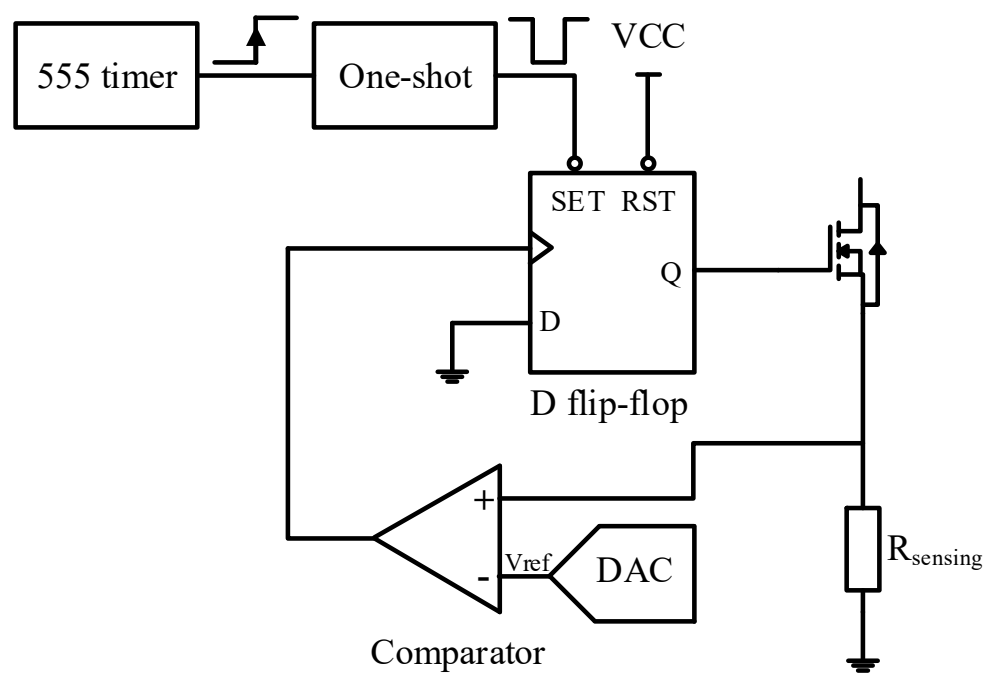

Fig. 3.6 Block diagram of control circuit.

\subsubsection{Simulation and Experiment}

The experimental setup in laboratory is shown in Fig. 3.7. The input DC voltage is $5 \mathrm{~V}$. A current probe CP30 and a high voltage differential probe CT3681 are used to measure the input current $i_{\text {in }}$ and output voltage $v_{o}$, respectively.

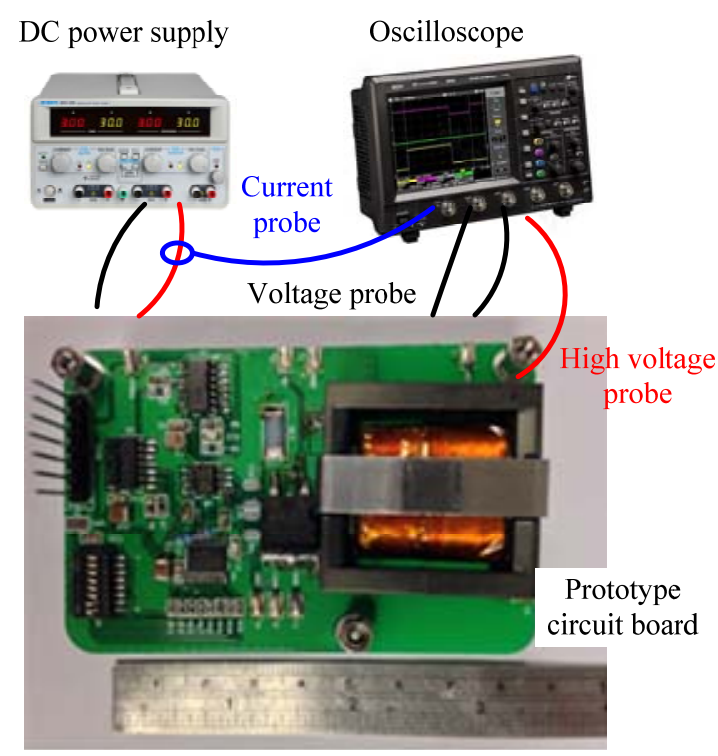

Fig. 3.7 Experimental setup of flyback circuit. 
To measure the potential maximum output voltage, the experiment is performed without connecting to the spark plugs of PPT igniter. As the input capacitor of high

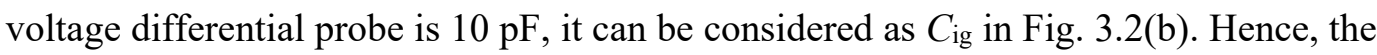
lumped capacitance $C_{r}$ is $2.98 \mathrm{nF}$ from (3.3) and Table 3.3.

Fig. 3.8 shows the experimental waveforms when the charging current limit $I_{\text {lim }}$ is $2.51 \mathrm{~A}$. The charging time is $51.3 \mu \mathrm{s}$. Once the MOSFET is turned off at time $t_{1}$, the circuit starts to resonate to generate a high voltage pulse. As the resonant angular frequency is as high as $2.11 \times 10^{6} \mathrm{rad} / \mathrm{s}$ according to transformer parameters listed in Table 3.3, the time duration for Modes 2 and 3 is less than $2 \mu \mathrm{s}$. Hence, $t_{1}, t_{2}$ and $t_{3}$ are labeled in the zoomed-in screenshot in the upper right corner. The measured pulse voltage is $3.7 \mathrm{kV}$.

It is noted that the input current $i_{\text {in }}$ reverses in Mode $4\left(t_{3}-t_{4}\right)$ since the resonance cannot transfer all the stored energy to $C_{\mathrm{ig}}$. Consequently, the flux in the transformer core is reset.

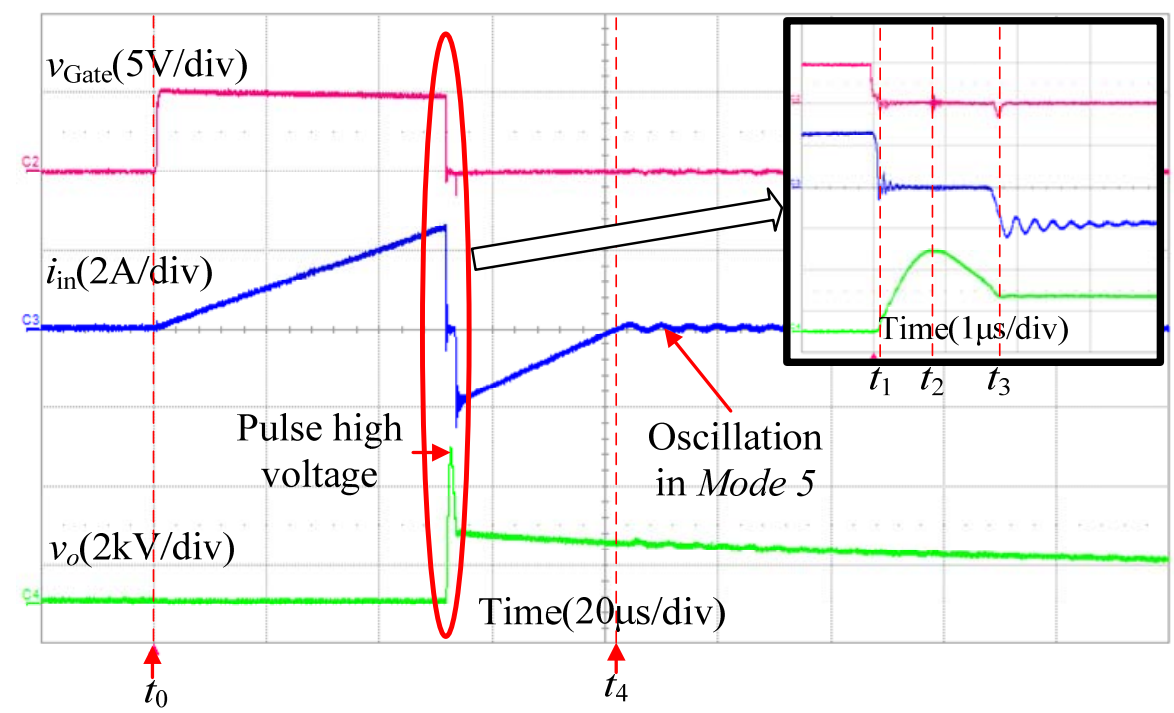

Fig. 3.8 Experimental waveforms of the flyback circuit. $v_{\text {Gate: }}$ gating signal of MOSFET, $i_{\text {in }}$ : input current, $v_{o}$ : the output voltage. 
To validate the output voltage regulation, experiment has been performed by adjusting the charging current limit $I_{\mathrm{lim}}$. Fig. 3.9 shows the maximum output voltage and the maximum repetitive frequency versus $I_{\mathrm{lim}}$. For comparisons, calculation and simulation results are also included. In the simulation, the parameters in Table 3.2 are used.

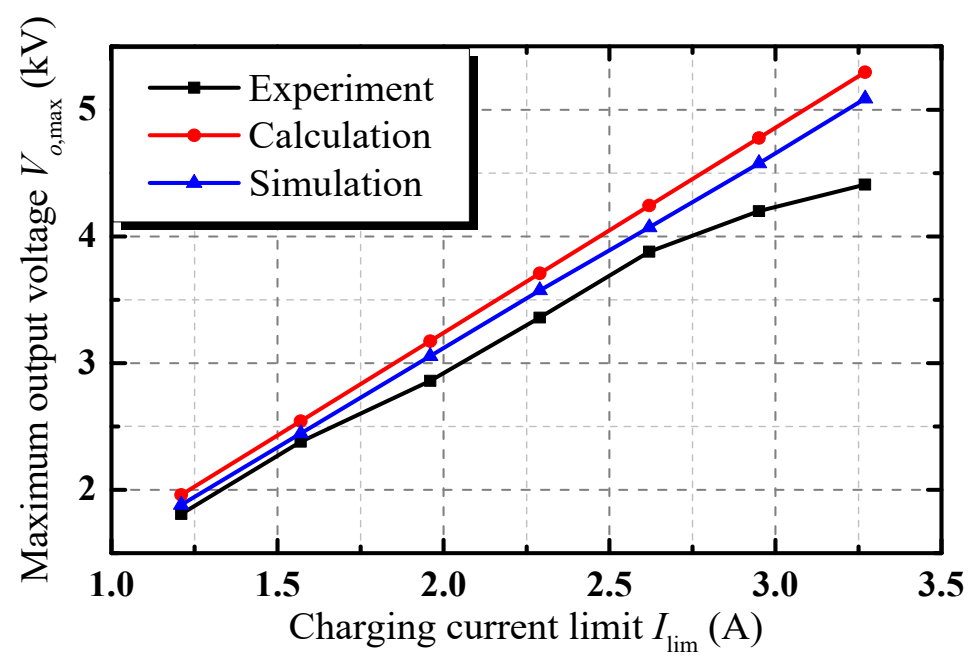

(a)

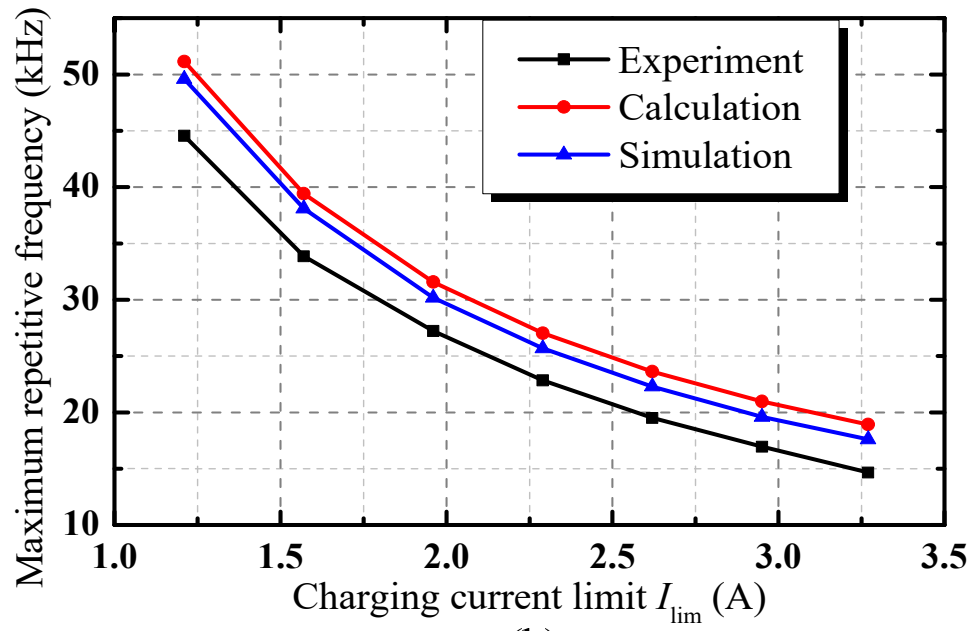

(b)

Fig. 3.9 (a) Maximum output voltage, and (b) maximum repetitive frequency versus charging current limit $I_{\text {lim. }}$. 
From Fig. 3.9(a), it is observed that the output voltage can be regulated by the charging current limit $I \mathrm{lim}$. The calculation is based on the theoretical analysis in section 3.3 given that all the components are ideal. Hence the theoretical results are the highest. In experiment, the voltage across MOSFET decreases with the increase in charging current limit. This is because more voltage drops on the current sensing resistor which is used to detect the current limit. This makes the MOSFET output capacitance $C_{\text {oss }}$ increase, enlarging the voltage error between the simulation and experiment according to (3.6) and (3.7). In Fig. 3.9(a), the largest error is $15.3 \%$, occurring at the charging current limit $I_{\text {lim }}$ of 3.27 A. From Fig. 3.9(b), the maximum repetitive frequency decreases with the increase in charging current limit.

The efficiency of flyback circuit with parasitic components has also been studied, and the results are shown in Fig. 3.10. The efficiency is defined as $W_{\text {Cig }} /\left(W_{\text {Cig }}+W_{\text {loss }}+W_{\text {ctrl }}\right) \cdot W_{\text {Cig }}$ is the energy stored in igniter capacitance $C_{\text {ig. }} . W_{\text {loss }}$ and $W_{\text {ctrl }}$ are the energy loss by the power components (transformer, MOSFET, diode and sensing resistor) and control circuits, respectively.

With different commercial integrated circuits, control circuits have various power consumption $P_{\text {ctrl. }}$ As the control circuits work effectively in Mode 1, the energy loss $W_{\text {ctrl }}$ is given by

$$
W_{\mathrm{ctrl}}=P_{\mathrm{ctrl}} T_{1}
$$

where $T_{1}$ is the time duration of Mode 1 given by (3.2). In this prototype, $P_{\text {ctrl }}$ is 26.3 mW typically.

From Fig. 3.10, it is observed that the overall efficiency of flyback circuit is $38.1 \%$ when the output voltage is $1.88 \mathrm{kV}$, and the efficiency decreases with the increase in output voltage. This is because a higher voltage stress on MOSFET results in more switching loss, and a higher charging current limit leads to more conduction loss. 


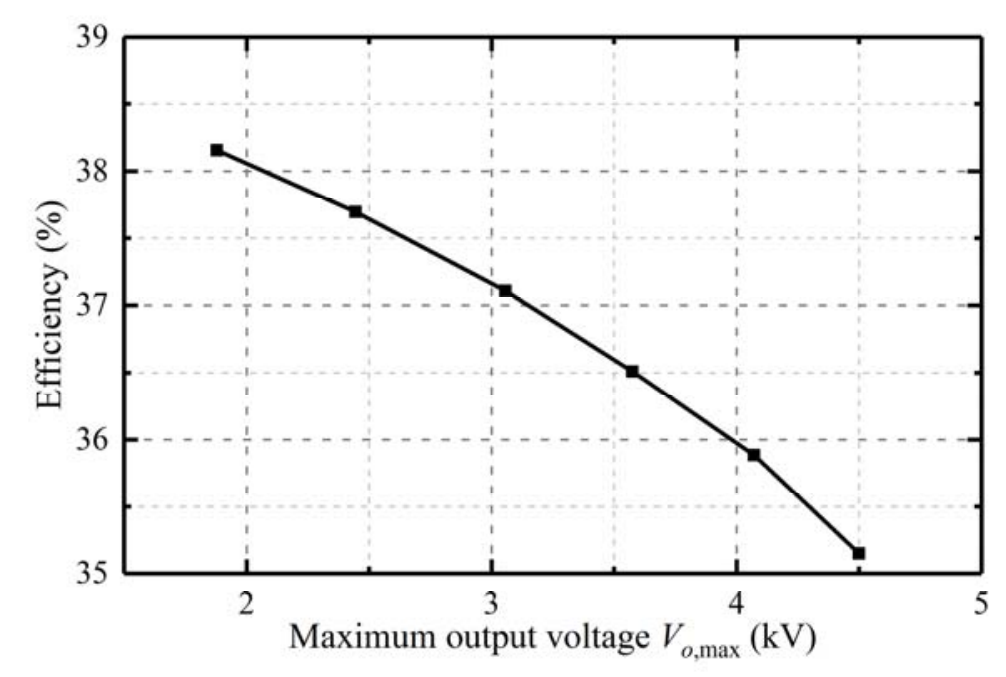

Fig. 3.10 Efficiency of the flyback circuit versus the maximum output voltage.

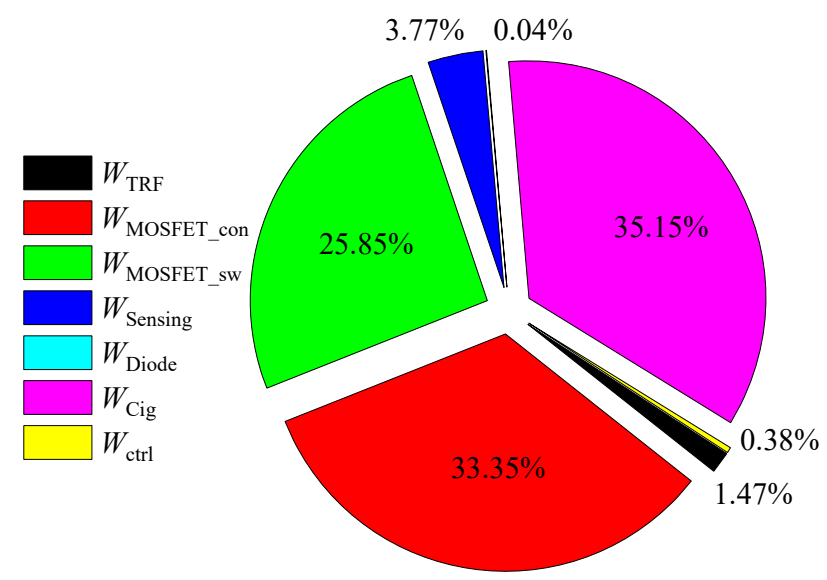

Fig. 3.11 Energy distribution when the flyback circuit outputs $4.5 \mathrm{kV}$.

Fig. 3.11 shows the energy distribution when the output voltage is $4.5 \mathrm{kV}$. The conduction loss $W_{\text {MOSFET_con }}$ and the switching loss $W_{\text {MOSFET_sw }}$ by MOSFET contribute $33.35 \%$ and $25.85 \%$, respectively. From the observation, the switching loss deteriorates the efficiency significantly. This is the nature drawback of hard-switching technique. As the circuit is proposed for low power applications, the conduction loss $W_{\text {Diode }}$ caused by diode is as low as $0.04 \%$. Because of the low DC resistance of transformer, the conduction loss $W_{\mathrm{TRF}}$ caused by transformer is $1.47 \%$. A current sensing resistor of 50 
$\mathrm{m} \Omega$ is used to monitor the charging current. It results in conduction loss $W_{\text {Sensing }}$ that contributes $3.77 \%$. $W_{\text {Cig }}$ is the energy transferred to $C_{\text {ig. }}$. Hence, the efficiency is $35.15 \%$ when the output voltage is $4.5 \mathrm{kV}$. The control circuits contributes $0.38 \%$ to the energy distribution.

To validate that the prototype circuit can ignite the plasma generation in space, an experiment has been conducted in the vacuum environment. The test setup and the experimental waveforms are shown in Figs. 3.12 and 3.13, respectively. The capacitor bank is charged to $1.5 \mathrm{kV}$, and the pulse frequency is set to $1 \mathrm{~Hz}$. The energy stored in the capacitor bank is released to form the main discharging when the prototype circuit supplies a high voltage of around $3 \mathrm{kV}$ to the PPT igniter. The discharging pulse current flowing through the surface of the Teflon propellant produces the plasma. The successful plasma generation in the vacuum environment is shown in Fig. 3.14.

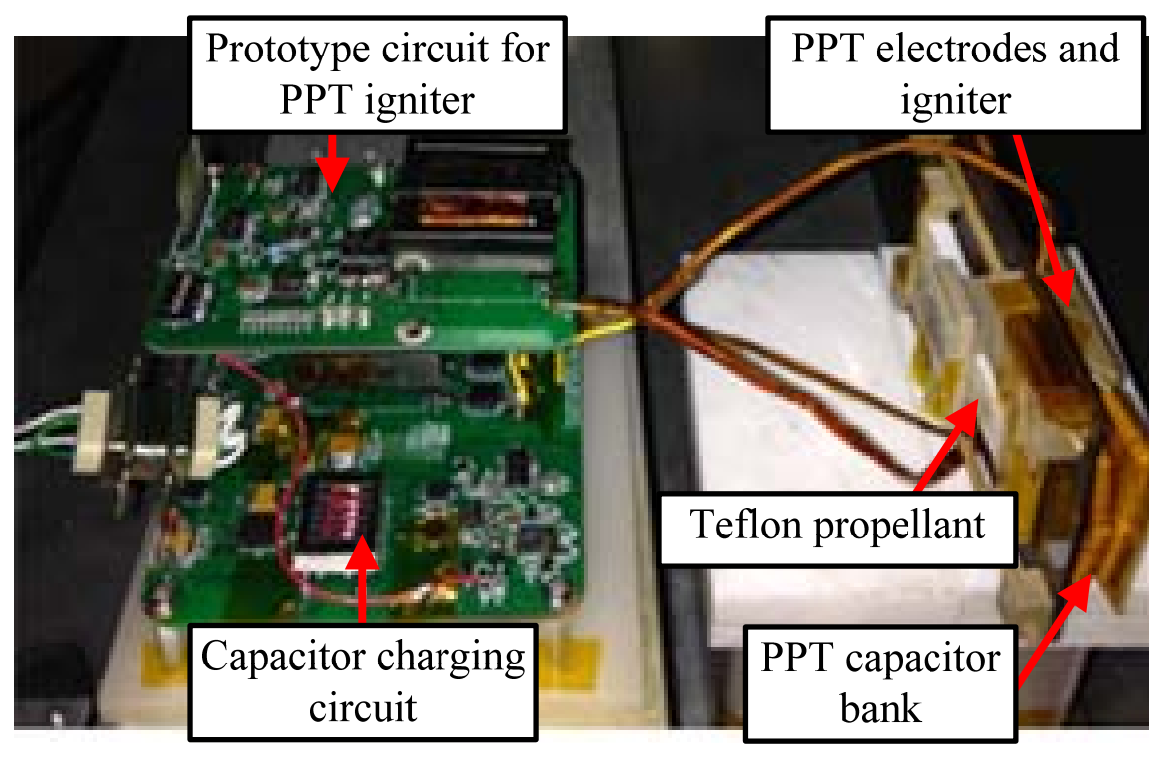

Fig. 3.12 PPT integration test in vacuum environment. 


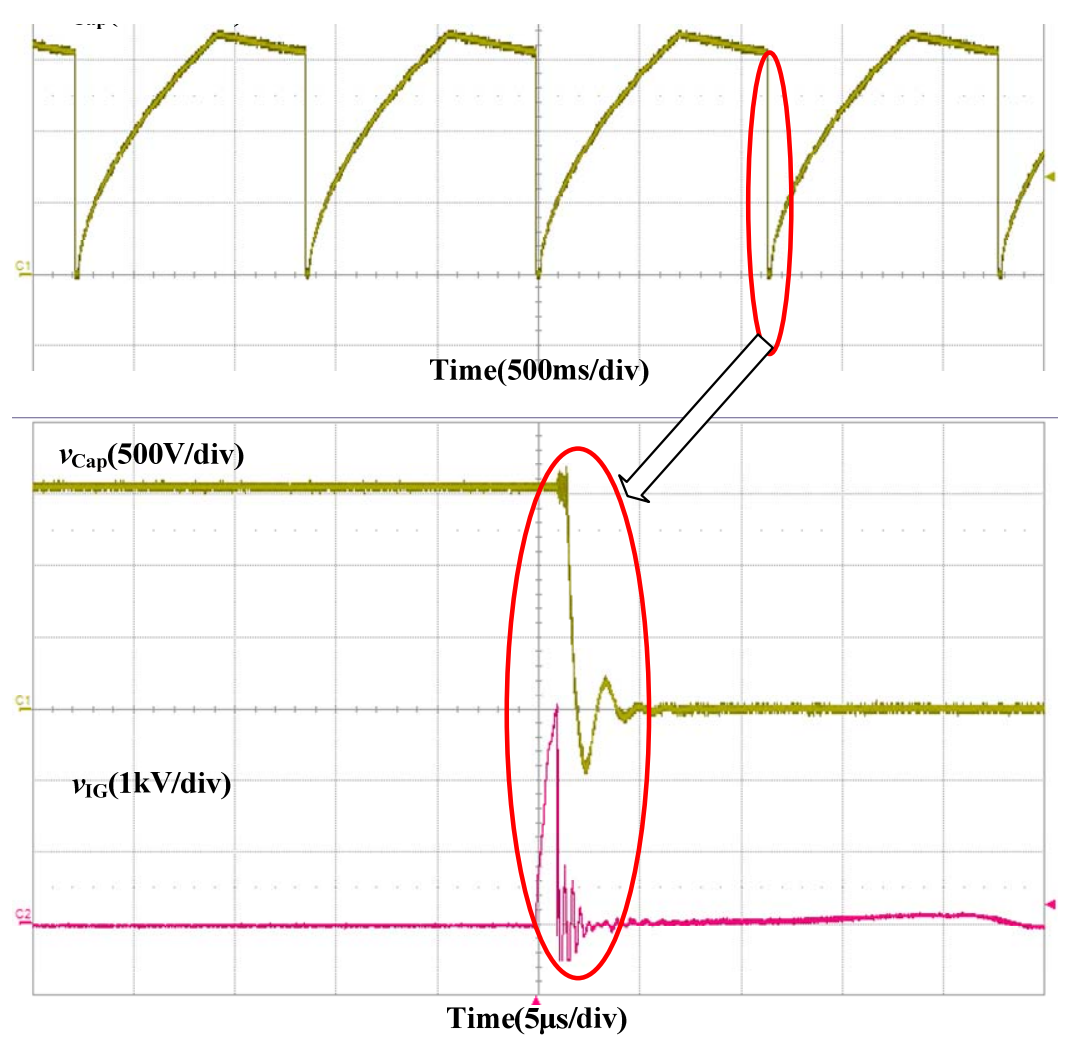

Fig. 3.13 Discharging waveforms. $v_{\text {Cap }}$ : voltage of PPT capacitor bank, $v_{\mathrm{IG}}$ : voltage of PPT igniter.

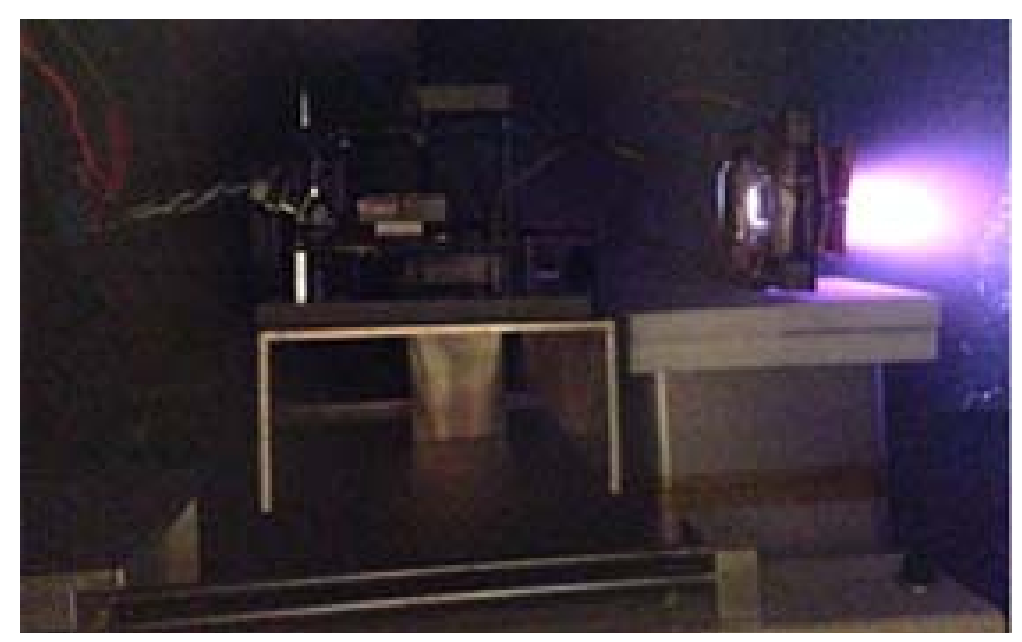

Fig. 3.14 Plasma generation in vacuum. 


\subsection{Summary}

This chapter presents a flyback circuit to generate high pulse voltage for a PPT igniter. The objective is to make use of the parasitic capacitances of components. In this way, an unreliable physical high voltage capacitor can be eliminated to improve the system reliability by $13.4 \%$. The high voltage is obtained from the resonance between the parasitic capacitances and transformer magnetizing inductance when the MOSFET is turned off.

The advantages of the circuit are small form factor and simple to control the switch. The theoretical analysis and prototype development have been presented. The simulation and experimental results have validated that the proposed topology can serve well as the PPT igniter circuit. However, the hard-switching nature of this circuit results in switching noise and switching loss, deteriorating power efficiency. In the next chapter, an igniter circuit using a soft-switching method will be presented to overcome these shortcomings. 


\section{Chapter 4}

\section{Single Switch Quasi-Resonant DC-DC Converter}

\subsection{Introduction}

From the literature review in Chapter 2, the soft-switching techniques are found useful to reduce switching loss and voltage spikes. This improves the reliability of semiconductor switches. However, all the resonant capacitors in the resonant tanks shown in Fig. 2.5 should be driven by a switch bridge which requires dead-time control to prevent short circuit of the upper and lower switches of the same leg. In practice, the use of multiple switches degrades the system reliability, and increases the circuit size. Compared to topologies based on a switch bridge, a quasi-resonant flyback converter can utilize a single switch. However, the valley-voltage-switching approach results in switching loss at light loads. Moreover, the input current is not quasi-sinusoidal. This may cause electromagnetic interference (EMI) issue.

The objective of this chapter is to propose a topology which can improve the voltage boost capability, reduce the number of power switches, ease the switching control, reduce the switching loss, and avoid the risk of EMI. If a quasi-sinusoidal input current can be achieved by a single switch, the resonant tank should consist of one series and two parallel resonant components at least, and is operated with zero-current-switching (ZCS).

Based on the considerations above, a quasi-resonant converter using a single switch as shown in Fig. 4.1 is proposed. The resonant tank consists of a series inductor $L_{\text {in, a }}$ 
shunt capacitor $C_{\mathrm{in}}$, and the transformer parameters including leakage inductance $L L_{\mathrm{k}}$, magnetizing inductance $L_{m}$ and winding capacitance $C_{w}$. Inherent the advantages of parallel resonant tank and series-parallel resonant tank, it has a good boost capability and output voltage regulation at light loads. Inductors $L_{\mathrm{k}}$ and $L_{m}$ provide the energy flow path for shunt capacitors $C_{\text {in }}$ and $C_{w}$. This allows the proposed resonant tank to be driven by a single switch. Hence, the switching control can be realized without a dead-time. Moreover, it eliminates the fault operation caused by single event upset in space or noise interference, which may result in short circuit in a switch bridge. Its smaller number of switches also makes it easier to incorporate a redundant circuit for better reliability.

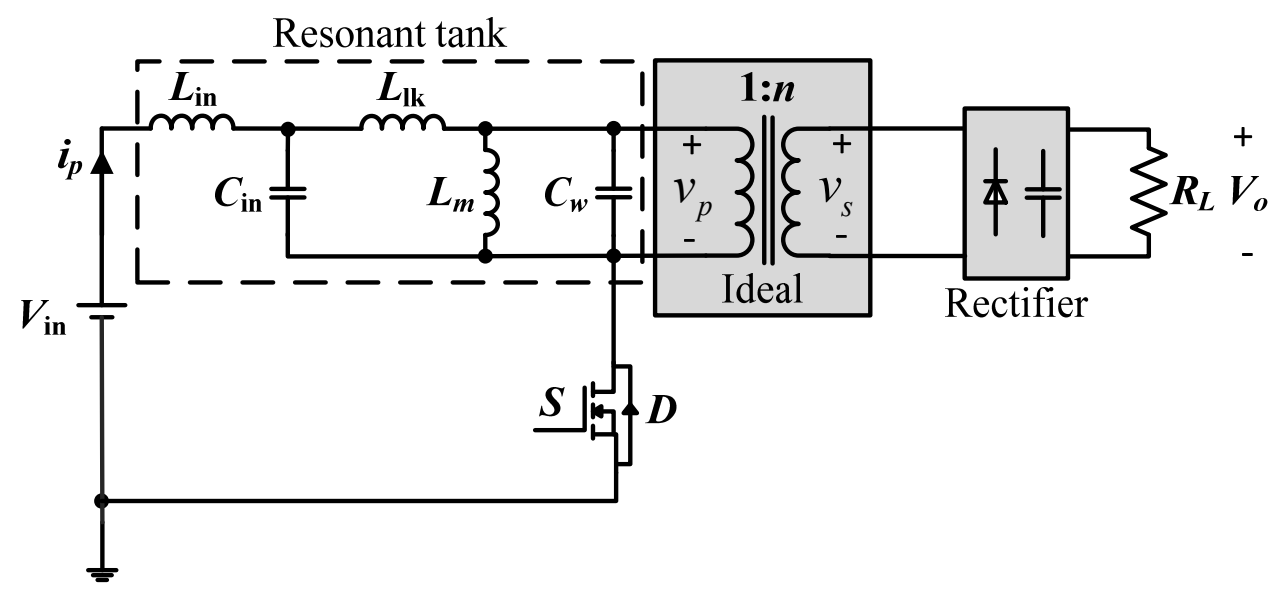

Fig. 4.1 Proposed quasi-resonant DC-DC converter using a single switch.

The switch in the proposed circuit can be turned on with zero-current (ZC), and turned off with zero-current zero-voltage $(\mathrm{ZCZV})$ to realize soft-switching. Besides reducing switch conduction loss, the switching frequency can be higher than half of the natural resonant frequency yielding a better boost capability as compared to the resonant converters operating with ZCS.

In this chapter, the details of this new quasi-resonant topology will be presented. The $\mathrm{AC}$ equivalent circuit is first discussed followed by the operating modes based on the 
approximated AC equivalent circuit. The mathematical analysis is then presented. To valid its performance, both simulation and experimental studies have been conducted.

\subsection{Principle of Operation}

\subsubsection{Model of a Transformer}

In [117], various models of a transformer have been reviewed, including the first-, second-, third-, and forth-order models. Among them, the third-order model shown in Fig. 4.2 is adopted to analyze the behavior of step-up transformer in high voltage applications $[118,119]$. The turns ratio of the primary winding to the secondary winding is $1: n$. With the parameters in the secondary side referred to the primary side, a step-up transformer is modeled by leakage inductance $L \mathrm{k}$, winding capacitance $C_{w}$ and magnetizing inductance $L_{m}$. All the three parameters form the proposed resonant tank. Hence, the adverse effects caused by transformer non-ideality such as voltage spikes can be mitigated.

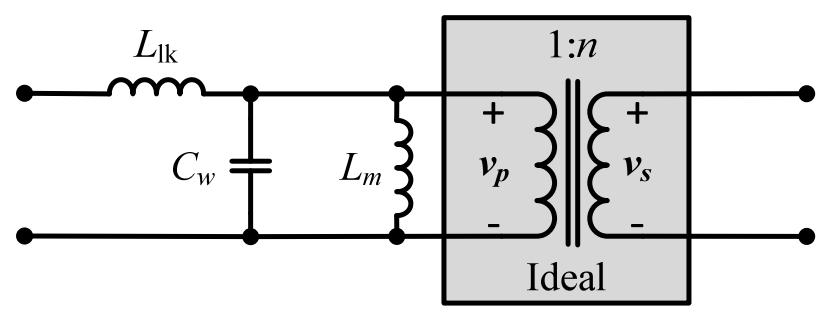

Fig. 4.2 Model of a step-up transformer.

The approach of extracting transformer parameters has been reported in [60]. From the transformer model in Fig. 4.2, magnetizing inductance $L_{m}$ and leakage inductance $L_{\mathrm{lk}}$ dominate the impedance at low switching frequency while winding capacitor $C_{w}$ has little effect if the secondary side is open. Hence, the impedance of primary side increases 
linearly with the switching frequency, i.e. $2 \pi f\left(L_{m}+L_{\mathrm{lk}}\right)$ where $f$ is the testing frequency. The resonance occurs between $L_{m}$ and $C_{w}$ where the impedance reaches the maximum, and the resonant frequency is $\frac{1}{2 \pi \sqrt{L_{m} C_{w}}}$. With the secondary winding short, $L_{\mathrm{lk}}$ makes the impedance of primary side increases linearly with the switching frequency, i.e. $2 \pi f L_{\mathrm{lk}}$. Using an LCR meter to scan the impedances with the secondary side open and short, the transformer parameters, $L_{m}, L_{\mathrm{lk}}$ and $C_{w}$, can be extracted.

\subsubsection{Cockcroft-Walton Voltage Multiplier}

As illustrated in Fig. 2.4, a rectifier circuit is required to invert the AC voltage from transformer to a DC output voltage. In high voltage applications such as the pulsed plasma thruster (PPT) igniter, it is an effective way to connect a few diodes in series to withstand high voltage. Instead of a diode bridge which is commonly used, a CockcroftWalton voltage multiplier (VM) shown in Fig. 4.3 is introduced in this study. This is because a VM circuit can boost the voltage using the same number of diodes when compared to a diode bridge $[36,50,55]$.

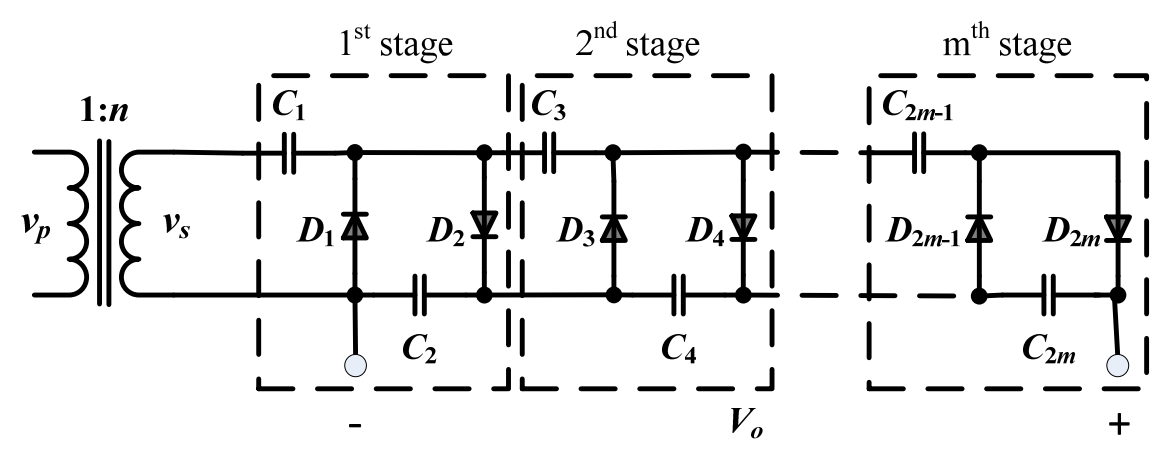

Fig. 4.3 Cockcroft-Walton voltage multiplier (VM).

In general, one stage of VM consists of two capacitors and two diodes. The output voltage $V_{o}$ of a VM circuit with $m$ stages is given by [36]: 


$$
V_{o}=m n V_{p, \mathrm{pk}-\mathrm{pk}}-\frac{I_{o}}{6 f_{s} C}\left(4 m^{3}+3 m^{2}+2 m\right)
$$

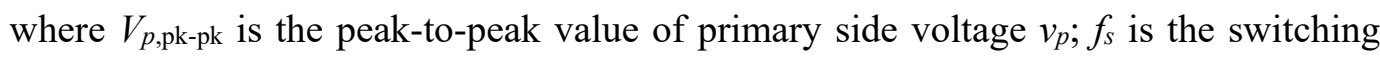
frequency; $I_{o}$ is the load current; and $C$ is the capacitance of $C_{1}, C_{2}, \ldots, C_{2 m-1}$ and $C_{2 m}$ used in the VM.

The load current $I_{o}$ for a load resistor $R_{L}$ is known by

$$
I_{o}=\frac{V_{o}}{R_{L}}
$$

By (4.1) and (4.2), the output voltage $V_{o}$ can be rewritten as

$$
V_{o}=\frac{m n V_{p, \mathrm{pk}-\mathrm{pk}}}{1+\frac{4 m^{3}+3 m^{2}+2 m}{6 R_{L} f_{s} C}}
$$

The output voltage of VM has a ripple $\delta V_{o}[36]$ :

$$
\delta V_{o}=\frac{V_{o}}{R_{L} f_{s} C} \cdot \frac{m(m+1)}{2} .
$$

This introduces capacitive element to the resonant operation. For light load applications such as PPT igniter, the voltage ripple in steady state can be neglected by selecting capacitance $C$ in VM properly to make the ripple negligible, e.g. $1 \%$ of the output voltage. By (4.4), the capacitance $C$ is larger than $\frac{m(m+1)}{1 \% \times\left(2 R_{L} f_{s}\right)}$. Hence, the VM can be modeled by an AC resistor.

Given that the VM is supplied by a sinusoidal voltage source and there is negligible energy loss, the AC equivalent resistance $R_{\mathrm{ac}}$ from the primary side of transformer can be expressed as

$$
\frac{\left(\frac{V_{p, \mathrm{pk}-\mathrm{pk}}}{2 \sqrt{2}}\right)^{2}}{R_{\mathrm{ac}}}=\frac{V_{o}^{2}}{R_{L}} .
$$


From (4.3) and (4.5), $R_{\mathrm{ac}}$ can be determined by

$$
R_{\mathrm{ac}}=\frac{R_{L}}{8(m n)^{2}}\left(1+\frac{4 m^{3}+3 m^{2}+2 m}{6 R_{L} f_{s} C}\right)^{2}
$$

\subsubsection{AC Equivalent Circuit}

A series inductor $L$ in and a shunt capacitor $C_{\text {in }}$ are added to adjust the range of switching frequency. The complete equivalent circuit is shown in Fig. 4.4(a). To analyze the characteristics of proposed topology, the AC equivalent circuit is simplified shown in Fig. 4.4. The П-type circuit formed by $C_{\mathrm{in}}, L_{\mathrm{lk}}$ and $C_{w}$ can be simplified to a T-type circuit formed by $L_{1}, C_{T}$ and $L_{2}$. $L_{\text {in }}$ and $L_{1}$ are lumped to $L_{s}$. The simplified third-order circuit can be obtained when the equivalent impedance of $L_{2}, L_{m}$ and $R_{\mathrm{ac}}$ in Fig. 4.4(b) is equal to that of $L_{T}$ and $R_{T}$ in Fig. 4.4(c). The details of derivation are given in Appendix A. In the sequel, the discussions on operating modes and analytical study are based on the approximated AC equivalent circuit shown in Fig. 4.4(c).

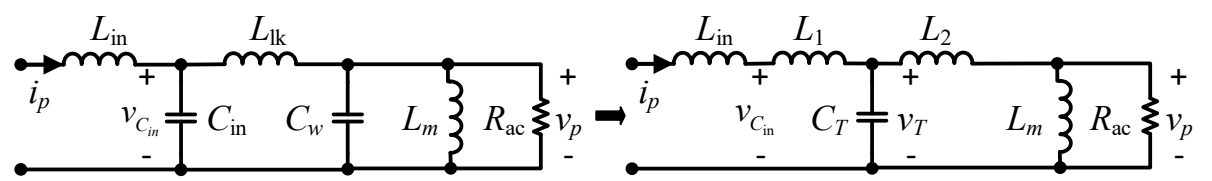

(a)

(b)

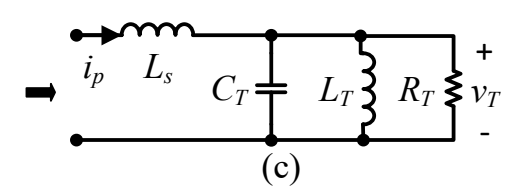

(c)

Fig. 4.4 Steps to simplify the AC equivalent circuit: (a) complete equivalent circuit with $\Pi$-type circuit $\left(C_{\mathrm{in}}, L_{\mathrm{lk}}, C_{w}\right),(\mathrm{b})$ equivalent circuit with T-type circuit $\left(L_{1}, C_{T}, L_{2}\right)$, and (c) approximated equivalent circuit. 


\subsubsection{Operating Modes}

In the proposed circuit, a metal-oxide-semiconductor field-effect transistor (MOSFET) with freewheeling diode $D$ is used as semiconductor switch $S$. The theoretical waveforms of proposed topology in steady state are illustrated in Fig. 4.5. To achieve zero-current-switching (ZCS), the MOSFET can be turned off anytime between $t_{1}$ and $t_{2}$ as in Fig. 4.5(a). The current goes through switch $S$ between $t_{0}$ and $t_{\text {off, and }}$ through freewheeling diode $D$ between $t_{\text {off }}$ and $t_{2}$. The results are identical anytime between $t_{1}$ and $t_{2}$ the switch is turned off given that switch $S$ and freewheeling diode $D$ are both ideal. In practice, the turn-off time affects the output voltage and switch conduction loss due to the non-ideality of MOSFET. This will be discussed in Section

\subsection{3.}

In the following discussion, the operating principle is described based on the case that the switch is turned off at time $t_{1}$ as in Fig. 4.5(b). Each switching cycle can be divided into three time intervals. The corresponding equivalent circuit for each mode is given in Fig. 4.6.

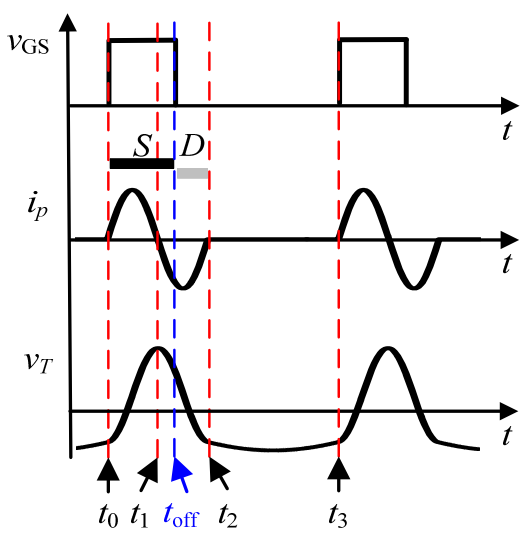

(a)

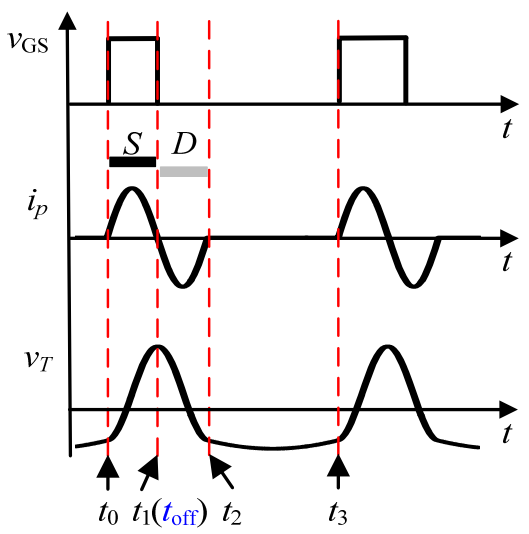

(b)

Fig. 4.5 Theoretical waveforms in steady state when the switch is turned off (a) between $t_{1}$ and $t_{2}$, and (b) at time $t_{1}, v_{\mathrm{GS}}$ : MOSFET gating signal, $i_{p}$ : primary side current, $v_{T}$ : lumped primary side voltage. 
1) Mode $1\left[t_{0}-t_{1}\right]$ : Switch $S$ (MOSFET) is turned on at time $t_{0}$ with a zero-current. The input power supply excites the resonant tank to work at quasi-resonant state resulting in $i_{p}$ and $v_{T}$ to be approximately sinusoidal. The voltage $v_{T}$ increases to the maximum within this time interval as energy is transferred to the resonant tank.

Inherent the advantages of parallel resonant converter (PRC) and series-parallel resonant converter (SPRC), the maximum value of $v_{T}$ can be higher than $V_{\text {in. Thus it }}$ exhibits boost capability. The primary side current $i_{p}$ turns zero the first time at $t_{1}$ when the MOSFET is turned off to achieve zero-current zero-voltage (ZCZV).

2) Mode $2\left[t_{1}-t_{2}\right]$ : If the voltage $v_{T}$ is higher than the input voltage $V_{\text {in }}$ at time $t_{1}$, the energy sustained in the resonant tank will flow back to the power supply. As a result, the quasi-resonant current reverses through freewheeling diode $D$ as shown in Fig. 4.6(b). It is observed from Figs. 4.6(a) and (b) that Modes 1 and 2 share the same equivalent circuit if the MOSFET and freewheeling diode are both ideal.

In Mode 2, the voltage $v_{T}$ decreases as some energy flows back to the power supply. The primary side current $i_{p}$ turns zero the second time at $t_{2}$. As $v_{T}$ decreases to smaller than $V$ in, the conduction path to the input power supply is blocked.

3) Mode $3\left[t_{2}-t_{3}\right]$ : The primary side current $i_{p}$ remains zero. The quasi-resonance occurs among $L_{T}, C_{T}$ and $R_{T}$ with a different resonant frequency.

Since Mode 3 is necessary for the quasi-resonant operation, the proposed topology is operated with discontinuous-current-mode (DCM). Hence, the maximum switching frequency should be smaller than the resonant frequency in Mode 1 (Mode 2). The time interval of Mode 3 will be smaller if the switching frequency is higher, resulting in the waveform of $v_{T}$ to be more sinusoidal. Moreover, the output voltage is higher as the switching frequency approaches to the natural resonant frequency. 
If switch $S$ is turned off at time $t_{2}$, the current $i_{p}$ goes through the switch in Mode 3 instead of the freewheeling diode. However, the operating modes are the same if the components are assumed to be ideal.

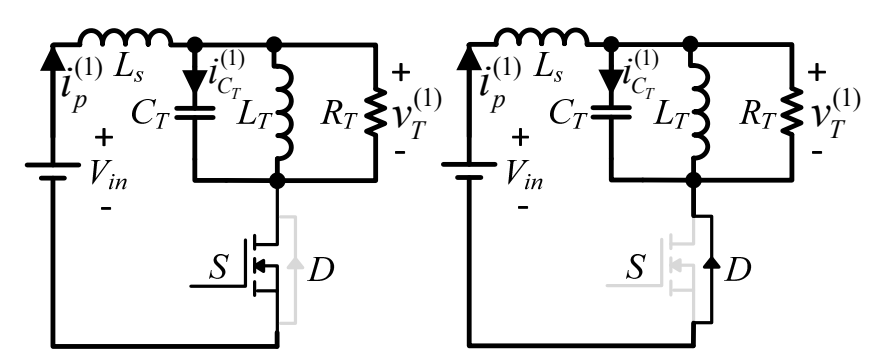

(a)

(b)

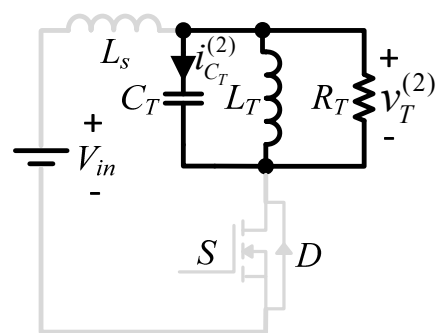

(c)

Fig. 4.6 Equivalent circuits for (a) Mode 1, (b) Mode 2, and (c) Mode 3 if the MOSFET is turned off at time $t_{1}$.

\subsubsection{Mathematical Analysis}

This theoretical analysis is conducted based on the discussions of operating modes. By the derived mathematical expressions of voltage and current, the performance of the proposed topology can be studied such as the voltage gain, turn-off time, output power, etc.

For ease of mathematical expression, the following parameters are defined:

$$
\omega_{1}=\frac{1}{\sqrt{L_{s} C_{T}}}, \omega_{2}=\frac{1}{\sqrt{L_{T} C_{T}}}, \tau=R_{T} C_{T}, \eta=\frac{L_{T}}{L_{s}+L_{T}}, L=\frac{L_{s} L_{T}}{L_{s}+L_{T}}
$$

State 1: Interval $t_{0}-t_{2}$ 
For Modes 1 and 2, they share the same equivalent circuit, and all the components participate in the quasi-resonance as shown in Figs. 4.6(a) and (b). By using the Kirchhoff's Current Law (KCL) and Kirchhoff's Voltage Law (KVL), the primary side current $i_{p}^{(1)}$, the lumped primary side voltage $v_{T}^{(1)}$ and the current of the lumped shunt resonant capacitor $i_{C_{T}}^{(1)}$ during $t_{0}-t_{2}$ can be expressed as

$$
\left[\begin{array}{c}
\frac{d i_{p}^{(1)}}{d t} \\
\frac{d v_{T}^{(1)}}{d t} \\
\frac{d i_{C_{T}}^{(1)}}{d t}
\end{array}\right]=\left[\begin{array}{ccc}
0 & -\frac{1}{L_{s}} & 0 \\
0 & 0 & \frac{1}{C_{T}} \\
0 & -\frac{1}{L} & -\frac{1}{\tau}
\end{array}\right]\left[\begin{array}{c}
i_{p}^{(1)} \\
v_{T}^{(1)} \\
i_{C_{T}}^{(1)}
\end{array}\right]+\left[\begin{array}{c}
-\frac{1}{L_{s}} \\
0 \\
\frac{1}{L_{s}}
\end{array}\right] V_{\mathrm{in}}
$$

where the superscript represents the state of the proposed topology during $t_{0}-t_{2}$ (Modes 1 and 2). Given that the start time $t_{0}=0$, the solution of the lumped primary side voltage $v_{T}^{(1)}$ for State 1 (Modes 1 and 2) is

$$
v_{T}^{(1)}(t)=e^{\alpha t}\left(M_{1} \cos \left(\beta_{1} t\right)+M_{2} \sin \left(\beta_{1} t\right)\right)+\eta V_{\text {in }}
$$

where $M_{1}$ and $M_{2}$ are constants related to the initial condition at $t=t_{0}$. The notations $\alpha$ and $\beta_{1}$ are

$$
\alpha=-\frac{1}{2 \tau}, \beta_{1}=\sqrt{\omega_{1}^{2}+\omega_{2}^{2}-\frac{1}{(2 \tau)^{2}}} .
$$

$\beta_{1}$ is the natural resonant angular frequency for State 1. By substituting (4.8) into (4.7), $i_{C_{T}}^{(1)}$ and $i_{p}^{(1)}$ are obtained:

$$
\left\{\begin{array}{l}
i_{C_{T}}^{(1)}(t)=C_{T} e^{\alpha t}\left(\left(M_{1} \alpha+M_{2} \beta_{1}\right) \cos \left(\beta_{1} t\right)+\left(M_{2} \alpha-M_{1} \beta_{1}\right) \sin \left(\beta_{1} t\right)\right) \\
i_{p}^{(1)}(t)=\frac{V_{\text {in }}}{L_{s}+L_{T}} t-\frac{1}{L_{s}}\left(M_{1} A_{1}(t)+M_{2} A_{2}(t)\right)
\end{array}\right.
$$

where 


$$
\left\{\begin{array}{l}
A_{1}(t)=\frac{e^{\alpha t}\left(\alpha \cos \left(\beta_{1} t\right)+\beta_{1} \sin \left(\beta_{1} t\right)\right)}{\alpha^{2}+\beta_{1}^{2}}-\frac{\alpha}{\alpha^{2}+\beta_{1}^{2}} \\
A_{2}(t)=\frac{e^{\alpha t}\left(\alpha \sin \left(\beta_{1} t\right)-\beta_{1} \cos \left(\beta_{1} t\right)\right)}{\alpha^{2}+\beta_{1}^{2}}+\frac{\beta_{1}}{\alpha^{2}+\beta_{1}^{2}}
\end{array}\right.
$$

In (4.10), the zero initial primary side current has been assumed, i.e. $i_{p}^{(1)}(0)=0$.

State 2: Interval $t_{2}-t_{3}$

State 2 represents the circuit behavior at Mode 3. From the Mode 3 equivalent circuit in Fig. 4.6(c), the circuit behavior can be described by

$$
\left[\begin{array}{c}
\frac{d v_{T}^{(2)}}{d t} \\
\frac{d i_{C_{T}}^{(2)}}{d t}
\end{array}\right]=\left[\begin{array}{cc}
0 & \frac{1}{C_{T}} \\
-\frac{1}{L_{T}} & -\frac{1}{\tau}
\end{array}\right]\left[\begin{array}{l}
v_{T}^{(2)} \\
i_{C_{T}}^{(2)}
\end{array}\right]
$$

where the superscript represents the state of the proposed topology during $t_{2}-t_{3}$ (Mode 3). The solution of $v_{T}^{(2)}(t)$ is

$$
v_{T}^{(2)}(t)=e^{\alpha\left(t-t_{2}\right)}\left(K_{1} \cos \left(\beta_{2}\left(t-t_{2}\right)\right)+K_{2} \sin \left(\beta_{2}\left(t-t_{2}\right)\right)\right)
$$

where the constants $K_{1}$ and $K_{2}$ depend on the condition at $t=t_{2}$. The notations $\beta_{2}$ is

$$
\beta_{2}=\sqrt{\omega_{2}^{2}-1 /(2 \tau)^{2}}
$$

Each operating cycle should be constrained by the boundary conditions, namely

$$
\left\{\begin{array}{l}
i_{C_{T}}^{(1)}\left(t_{2}\right)=i_{C_{T}}^{(2)}\left(t_{2}\right) \\
i_{C_{T}}^{(1)}(0)=i_{C_{T}}^{(2)}(T) \\
v_{T}^{(1)}\left(t_{2}\right)=v_{T}^{(2)}\left(t_{2}\right) \\
v_{T}^{(1)}(0)=v_{T}^{(2)}(T)
\end{array}\right.
$$

where $T$ is the switching period. Define the variables $X_{1}, Y_{1}, X_{2}$ and $Y_{2}$ as

$$
\left\{\begin{array}{l}
X_{1}=e^{\alpha t_{2}} \cos \left(\beta_{1} t_{2}\right) \\
Y_{1}=e^{\alpha t_{2}} \sin \left(\beta_{1} t_{2}\right) \\
X_{2}=e^{\alpha\left(T-t_{2}\right)} \cos \left(\beta_{2}\left(T-t_{2}\right)\right) \\
Y_{2}=e^{\alpha\left(T-t_{2}\right)} \sin \left(\beta_{2}\left(T-t_{2}\right)\right)
\end{array} .\right.
$$


The boundary relationships of current and voltage can be arranged as

$$
\left[\begin{array}{cccc}
-1 & 0 & X_{2} & Y_{2} \\
0 & \beta_{1} & \beta_{2} Y_{2} & -\beta_{2} X_{2} \\
X_{1} & Y_{1} & -1 & 0 \\
\beta_{1} Y_{1} & -\beta_{1} X_{1} & 0 & \beta_{2}
\end{array}\right]\left[\begin{array}{c}
M_{1} \\
M_{2} \\
K_{1} \\
K_{2}
\end{array}\right]=\eta V_{\text {in }}\left[\begin{array}{c}
1 \\
\alpha \\
-1 \\
-\alpha
\end{array}\right] .
$$

Using, (4.16) and (4.17), $M_{1}, M_{2}, K_{1}$ and $K_{2}$ can be expressed by $t_{2}$. The primary side current $i_{p}$ is zero at $t_{2}$. Hence, $t_{2}$ can be calculated by solving $i_{p}^{(1)}\left(t_{2}\right)=0$. The time $t_{1}$ when $i_{p}$ turns zero the first time is the other solution to the equation $i_{p}^{(1)}(t)=0$. The peak-to-peak value of primary side voltage $V_{p, \text { pk-pk }}$ and the output voltage $V_{o}$ are known by (A13) and (4.1), respectively.

While the transcendental equations are complex to derive a closed form of expressions, they can be solved using numerical method if the values of all the components and switching frequency are given. Fig. 4.7 shows the flow chart to calculate $t_{2}$ numerically. CurrRes is a pre-defined parameter. The current is considered to be approximately zero if $a b s\left(i_{p}^{(1)}(t)\right)<$ CurrRes . $a b s\left(i_{p}^{(1)}(t)\right)$ is the absolute value of $i_{p}^{(1)}(t)$. As discussed in Section 3.2.4, the lumped primary side voltage $v_{T}$ at time $t_{2}$ is smaller than the input voltage $V_{\text {in. }}$ Using this condition, $t_{2}$ can be determined. 


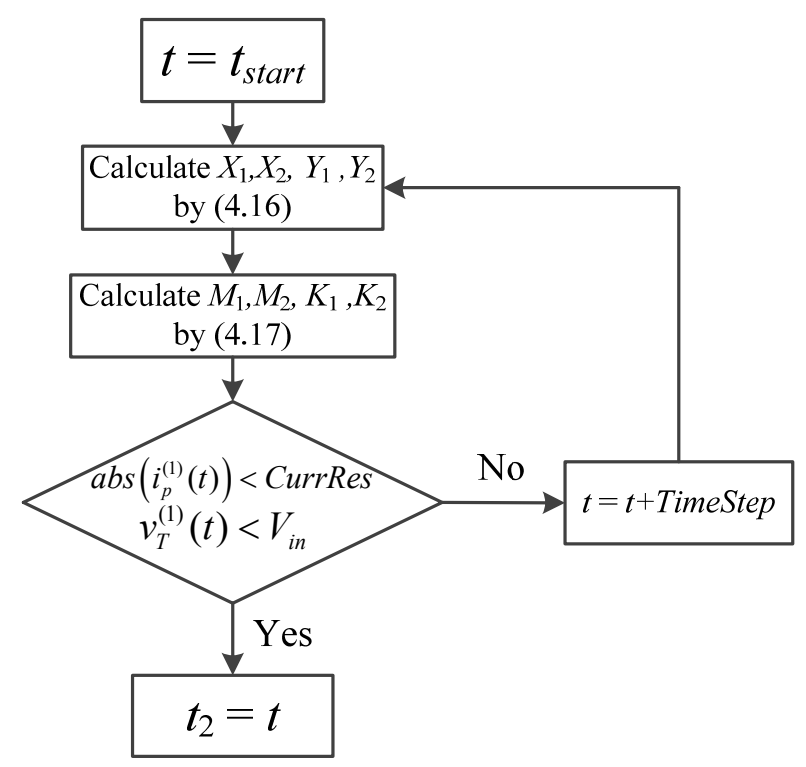

Fig. 4.7 Flow chart to calculate $t_{2}$ numerically.

Due to the complexity of resonant tank shown in Fig. 4.4(a), the topology parameters $\left(L_{s}, C_{T}, L_{T}, f_{n}\right)$ in the approximated $\mathrm{AC}$ equivalent circuit shown in Fig. 4.4(c) are first investigated. Using the numerical approach, the normalized voltage gain $v_{n}$ with respect to these parameters is shown in Fig. 4.8. It can be observed that:

- An increase in normalized switching frequency $f_{n}$ leads to a higher normalized voltage gain $v_{n}$.

- The lumped shunt capacitance $C_{T}$ has no significant effect on $v_{n}$.

- A decrease in lumped series inductance $L_{s}$ or an increase in lumped shunt resonant inductance $L_{T}$ makes $v_{n}$ increase.

- The component selection has constraints for ZCS operation. A higher normalized switching frequency $f_{n}$ has a wider range. Moreover $C_{T}$ affects little on the range of $L_{s}$ while a larger $L_{T}$ can result in a wider range of $L_{s}$. 


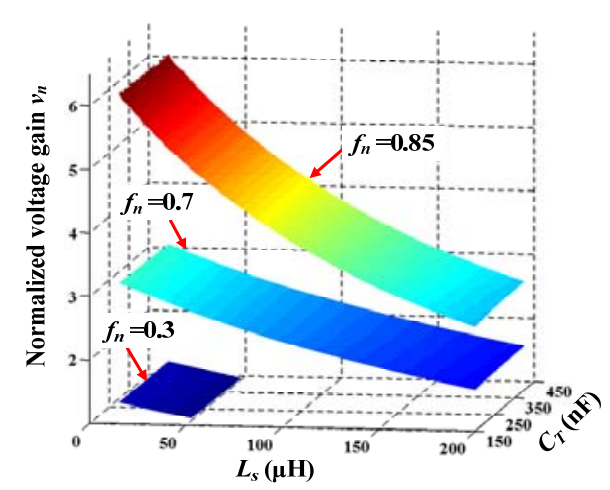

(a)

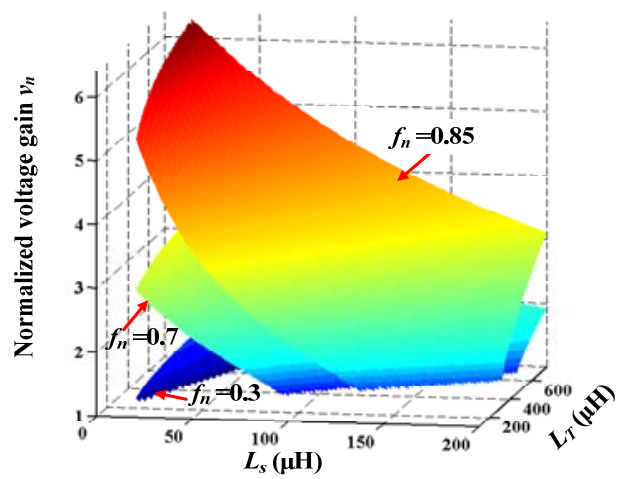

(b)

Fig. 4.8 Normalized voltage gain $v_{n}$ with respect to (a) $L_{m}=600 \mu \mathrm{H}, L_{\mathrm{lk}}=0.6 \mu \mathrm{H}, R_{L}=100 \mathrm{M} \Omega$; (b) $C_{T}$ $=264.3 \mathrm{nF}, L_{\mathrm{lk}}=0.6 \mu \mathrm{H}, R_{L}=100 \mathrm{M} \Omega$.

The root-mean-square (RMS) of primary side current $i_{p}$ is shown in Fig. 4.9. From the figure, it is observed that:

- The RMS of primary side current $i_{p}$ increases with the increase in normalized switching frequency $f_{n}$.

- Smaller value of $C_{T}, L_{T}$ or larger value of $L_{s}$ results in a smaller RMS of $i_{p}$. This is helpful in reducing the power loss. Hence, there is a tradeoff in selection of components considering the switching frequency range, voltage gain, circulating current and ZCS operation.

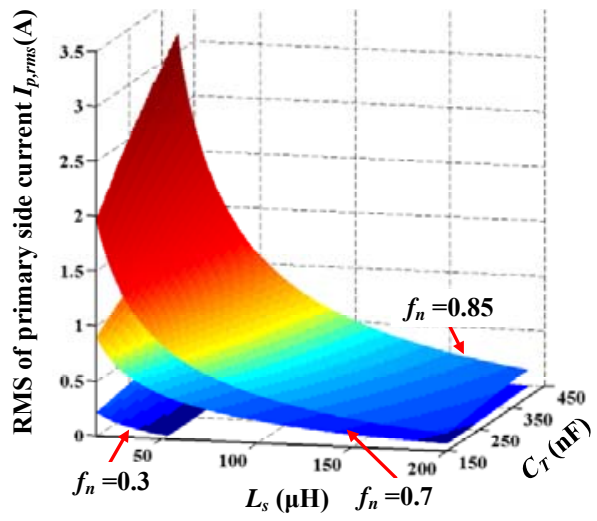

(a)

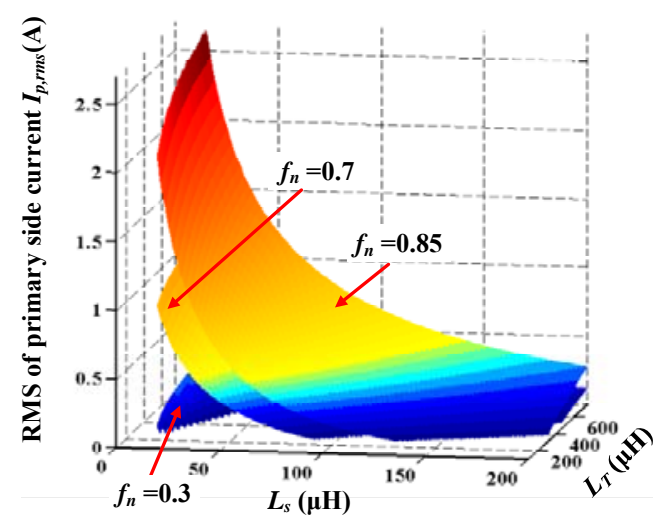

(b)

Fig. 4.9 Root-mean-square of $i_{p}$ with (a) $L_{m}=600 \mu \mathrm{H}, L_{1 \mathrm{k}}=0.6 \mu \mathrm{H}, R_{L}=100 \mathrm{M} \Omega$; (b) $C_{T}=264.3 \mathrm{nF}$, $L_{\mathrm{lk}}=0.6 \mu \mathrm{H}, R_{L}=100 \mathrm{M} \Omega$. 
From the approximation in Appendix A, Table 4.1 concludes the parameter relationships between the complete and approximated resonant tanks. Hence, the effect of parameters in the complete resonant tank can be known qualitatively. For example, the increase in $L_{\text {in }}$ makes $L_{s}$ increase from Table 4.1, resulting in the decrease in normalized voltage gain and RMS of primary side current according to Figs. 4.8 and 4.9, respectively.

Table 4.1 Parameter relationships between complete and approximated resonant tanks

\begin{tabular}{cccc}
\hline \hline & $L_{s}$ & $L_{T}$ & $C_{T}$ \\
\hline$L_{\text {in } \uparrow} \uparrow$ & $\uparrow$ & - & - \\
$C_{\text {in }} \uparrow$ & $\downarrow$ & $\uparrow$ & $\uparrow$ \\
$L_{\mathrm{lk}} \uparrow$ & $\uparrow$ & $\uparrow$ & $\downarrow$ \\
$C_{w} \uparrow$ & $\uparrow$ & $\downarrow$ & $\uparrow$ \\
$L_{m} \uparrow$ & - & $\uparrow$ & - \\
\hline \hline
\end{tabular}

\subsection{Simulation and Experimental Results}

To validate the proposed concept and theoretical analysis discussed in Section 4.2, this section presents the simulation study and experimental results.

\subsubsection{Components Used in Prototype Circuit}

Table 4.2 lists the components and parameters of transformer used in the simulation and prototype circuit. The transformer is custom made in laboratory using a ferrite core B66423GX187. Considering the voltage gain and transformer design, a single layer of primary winding is coiled, and covers the length of bobbin. Limited by the window area of ferrite core, the primary winding has 15 turns while the secondary winding has 450 turns. In our laboratory, an LCR meter E4098A is used to measure the impedance of 
primary winding with the secondary winding open and short, and the transformer parameters are extracted using the approach in Section 4.2.1. As shown in Fig. 4.8, a series inductor of less than $51.6 \mu \mathrm{H}$ can obtain the proposed zero-current-switching (ZCS) by a low switching frequency, e.g. $f_{n}=0.3$. In this study, an inductor of $33 \mu \mathrm{H}$ is used. A capacitor of $200 \mathrm{nF}$ is introduced to limit the switching frequency to less than $54 \mathrm{kHz}$. This helps to avoid the electromagnetic interference (EMI) from the gating signal which is a square wave, and can generate a wide range of spectrums.

Table 4.2 Components in simulation and prototype

\begin{tabular}{|c|c|c|}
\hline \multirow{2}{*}{$\begin{array}{c}\text { Added } \\
\text { components }\end{array}$} & Inductor, $L_{\mathrm{in}}$ & $\begin{array}{c}33 \mu \mathrm{H} \\
\text { MSS1260T-333MLB }\end{array}$ \\
\hline & Capacitor, $C_{\text {in }}$ & $\begin{array}{c}220 \mathrm{nF} / 50 \mathrm{~V} \\
\mathrm{C} 4532 \mathrm{C} 0 \mathrm{G} 1 \mathrm{H} 224 \mathrm{~J} 320 \mathrm{KA}\end{array}$ \\
\hline \multirow{5}{*}{ Transformer } & Ferrite core & B66423GX187 \\
\hline & Magnetizing inductance, $L_{m}$ & $600 \mu \mathrm{H}$ \\
\hline & Leakage inductance, $L_{\mathrm{lk}}$ & $0.6 \mu \mathrm{H}$ \\
\hline & Winding capacitor, $C_{w}$ & $44.3 \mathrm{nF}$ \\
\hline & Turns ratio, $n$ & $15: 450$ \\
\hline \multirow{3}{*}{$\begin{array}{l}\text { Voltage } \\
\text { multiplier }\end{array}$} & Number of stages, $m$ & 8 \\
\hline & Capacitor, $C$ & $\begin{array}{c}10 \mathrm{nF} / 3 \mathrm{kV} \\
1825 \mathrm{HC} 103 \mathrm{KAT} 1 \mathrm{~A}\end{array}$ \\
\hline & Diode part number & SF1600-TR-E3/1.6kV \\
\hline \multirow{4}{*}{ MOSFET } & Part number & AP40T03GH-HF-3TR \\
\hline & Drain-source on-resistance $R_{\mathrm{on}}$ & $45 \mathrm{~m} \Omega$ \\
\hline & Freewheeling diode threshold voltage $V_{\mathrm{SD}}$ & $0.65 \mathrm{~V}$ \\
\hline & Freewheeling diode on-resistance $R_{\mathrm{SD}}$ & $125 \mathrm{~m} \Omega$ \\
\hline
\end{tabular}

\subsubsection{Validating Approximated Equivalent Circuit}

As aforementioned discussion, it is complicated if the complete AC circuit shown in Fig. 4.4(a) is used for the theoretical analysis. Instead, an approximated third-order circuit shown in Fig. 4.4(c) is adopted to study the circuit quasi-resonant operation at light loads. To validate the approximation, the results of simulation and theoretical calculations will be compared. 
Fig. 4.10 shows the simulation results using the circuit shown in Fig. 4.1, and theoretical calculation based on the approximated equivalent circuit discussed in Section 4.2.5. A light load of $100 \mathrm{M} \Omega$ is used in Fig. 4.10(a). The full load for the proposed topology using ZCS varies between $14.8 \mathrm{M} \Omega$ and 17.7 $\mathrm{M} \Omega$ from Fig. 4.18. Hence, a nearly full load of $18 \mathrm{M} \Omega$ is used in Fig. 4.10(b).

Define the percentage absolute error $\varepsilon$ as

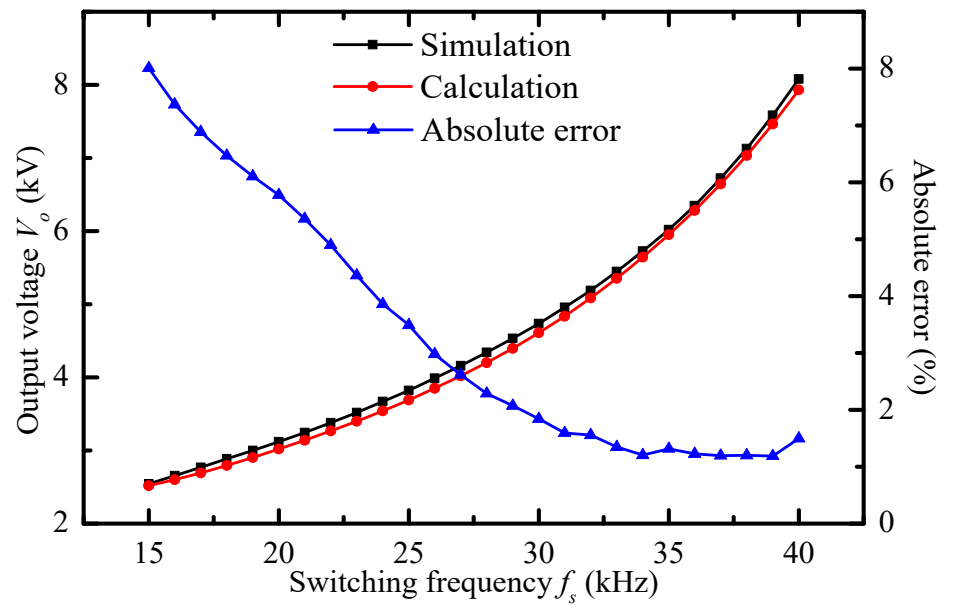

(a)

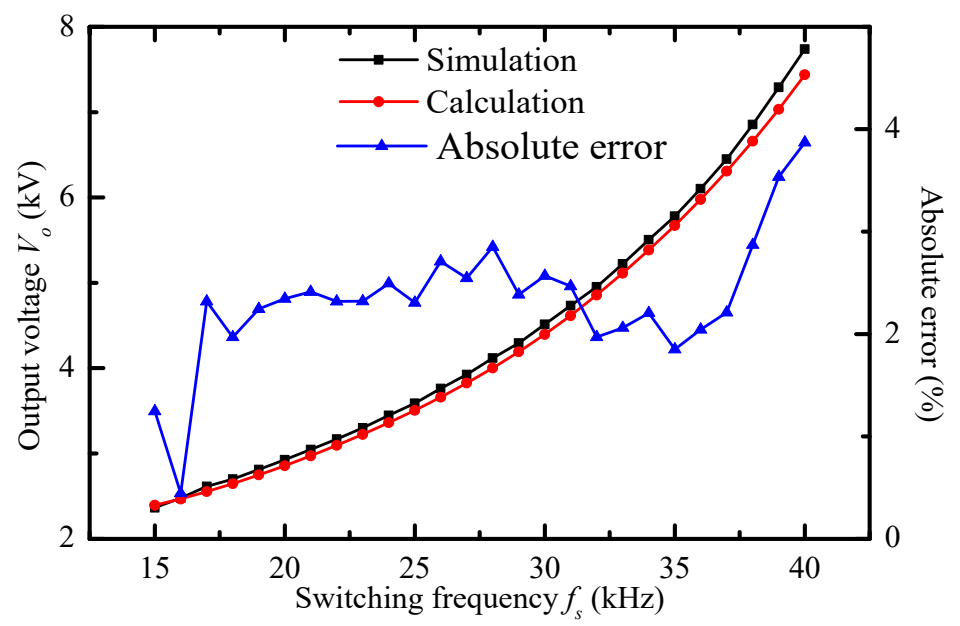

(b)

Fig. 4.10 Comparisons on output voltage between simulation and calculation results. Load is (a) 100 $\mathrm{M} \Omega$, and (b) $18 \mathrm{M} \Omega$. 


$$
\varepsilon=\frac{a b s(\text { Simulation }- \text { Calculation })}{\text { Simulation }} \times 100 \%
$$

From Fig. 4.10(a), $\varepsilon$ is less than $8.1 \%$ with a load of $100 \mathrm{M} \Omega$ at the switching frequency range $15 \mathrm{kHz}<f_{s}<40 \mathrm{kHz}\left(0.278<f_{n}<0.741\right)$. From Fig. $4.10(\mathrm{~b}), \varepsilon$ is less than $4.1 \%$ with a load of $18 \mathrm{M} \Omega$. Hence, the approximated AC equivalent circuit in Fig. 4.6(c) is acceptable for the analytical analysis of proposed topology within the full range of load. The error source mainly comes from the model simplification. The AC equivalent circuit of proposed topology approximated in Appendix A is based on the assumption that current and voltage are sinusoidal. Hence, it can be observed that from Fig. 4.10(a) that the error becomes smaller with an increase in switching frequency. This is because the sinusoidal distortion is relieved when a higher switching frequency makes the time interval $t_{2}-t_{3}$ in Fig. 4.5 smaller. On the other hand, the VM is simplified as a resistor at light loads. However, the voltage ripple will start to introduce capacitive component due to the increase in output power. This affects the quasi-resonant operation, making the error in Fig. 4.10(b) tends to increase when the switching frequency is higher than $36 \mathrm{kHz}$.

Fig. 4.11 shows the comparisons on $t_{1} / T$. $\varepsilon$ is less than $6.1 \%$ with a load of $100 \mathrm{M} \Omega$ in Fig. 4.11(a), while $\varepsilon$ is less than $10 \%$ with a load of $18 \mathrm{M} \Omega$ in Fig. 4.11(b). From this viewpoint, the switching control needs the help of simulation. To reduce the error, a more accurate model should be investigated considering the states of diodes in VM. Nevertheless, the approximated AC equivalent circuit can be used to predict the voltage gain. 


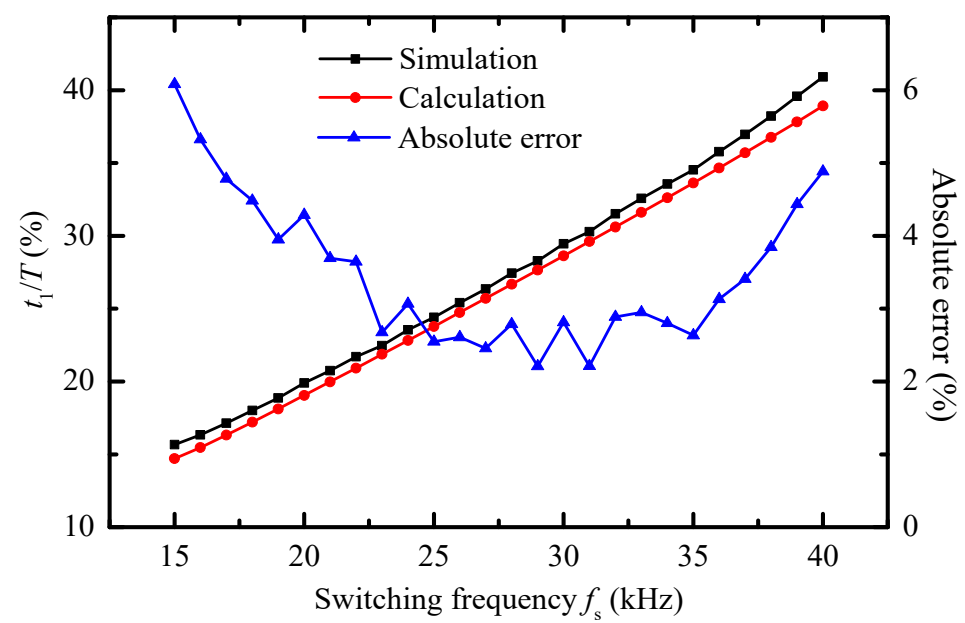

(a)

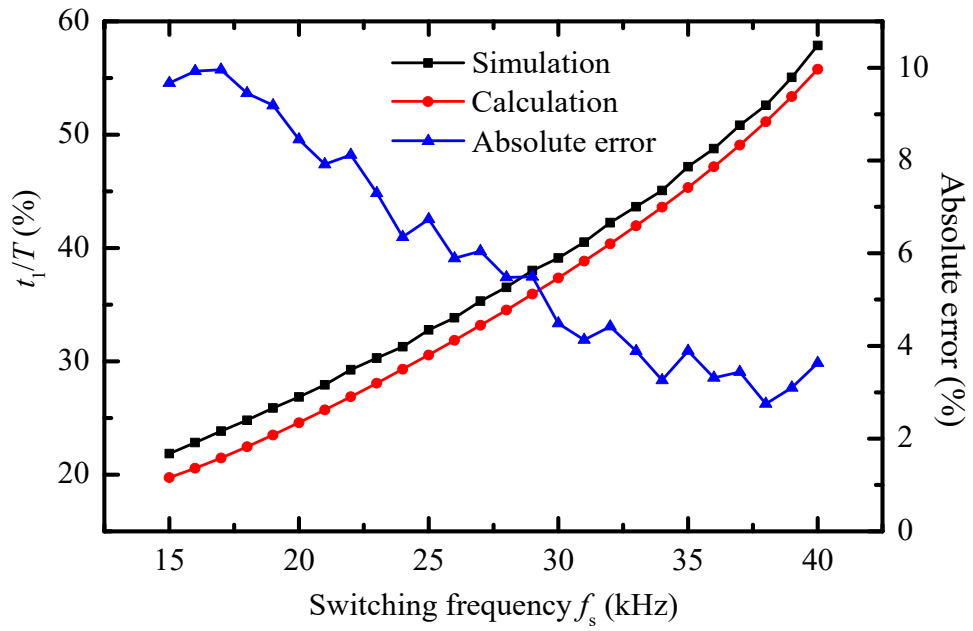

(b)

Fig. 4.11 Comparisons on $t_{1} / T$ between simulation and calculation results. Load is (a) $100 \mathrm{M} \Omega$, and (b) $18 \mathrm{M} \Omega$.

\subsubsection{Experimental Results}

A prototype circuitry shown in Fig. 4.12(a) has been developed to validate the proposed converter. The experimental setup is shown in Fig. 4.12(b). A high voltage probe $\mathrm{HVP}-15 \mathrm{HF}$ is used to measure the output voltage, and a differential voltage probe CT3681 is used to measure the voltage $v_{\mathrm{C}_{\mathrm{in}}}$ on capacitor $C_{\mathrm{in}}$. The primary side current $i_{p}$ is measured by a current probe $\mathrm{CP} 030$. 


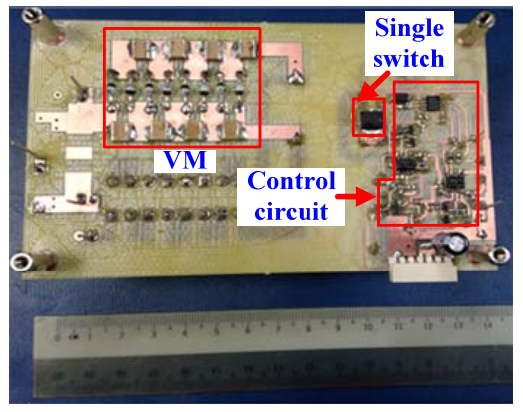

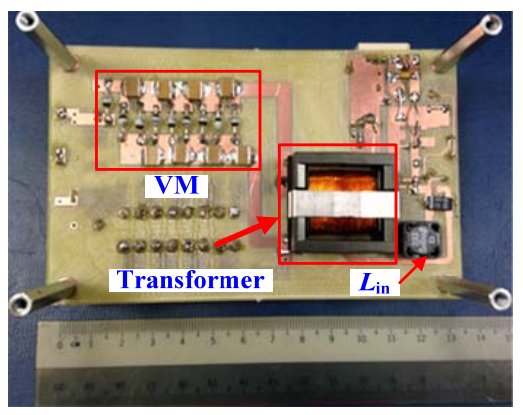

(a)

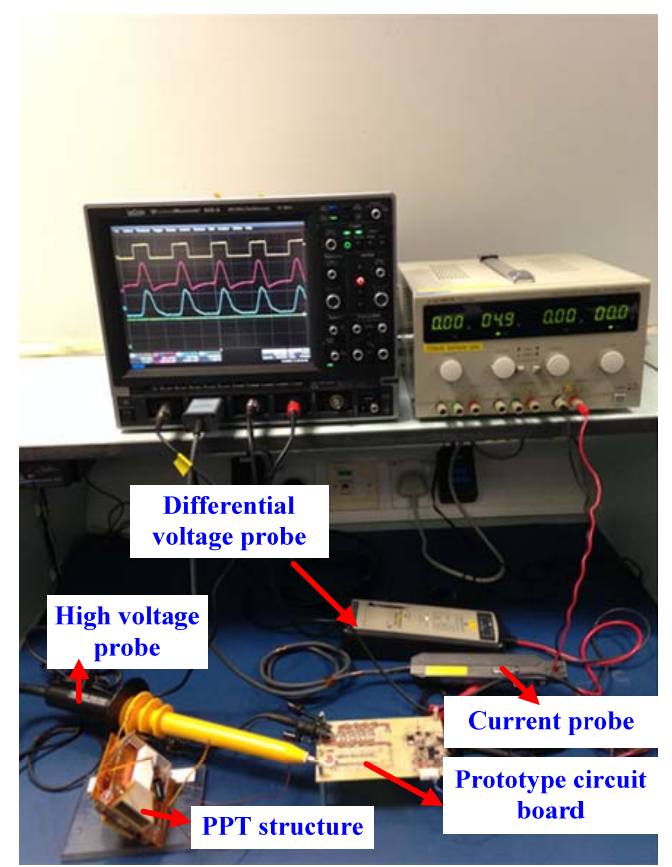

(b)

Fig. 4.12 (a) Prototype circuit board. (b) Experimental test setup.

Figs. 4.13 and 4.14 show the experimental results to validate the soft-switching operation. The switch is set to turn off at time $t$. From the waveforms, it is observed that the switching frequency is $26 \mathrm{kHz}\left(f_{n}=0.48\right)$, and the switching duty cycle $t_{1} / \mathrm{T}$ is $28.3 \%$. From Appendix A, the amplitude ratio of $v_{T}$ to $v_{\mathrm{C}_{\mathrm{in}}}$ is 0.9997 because of the small leakage inductance $(0.6 \mu \mathrm{H})$. Hence, the experimental voltage waveforms on $C_{\text {in }}$ are labeled as $v_{T}$. 


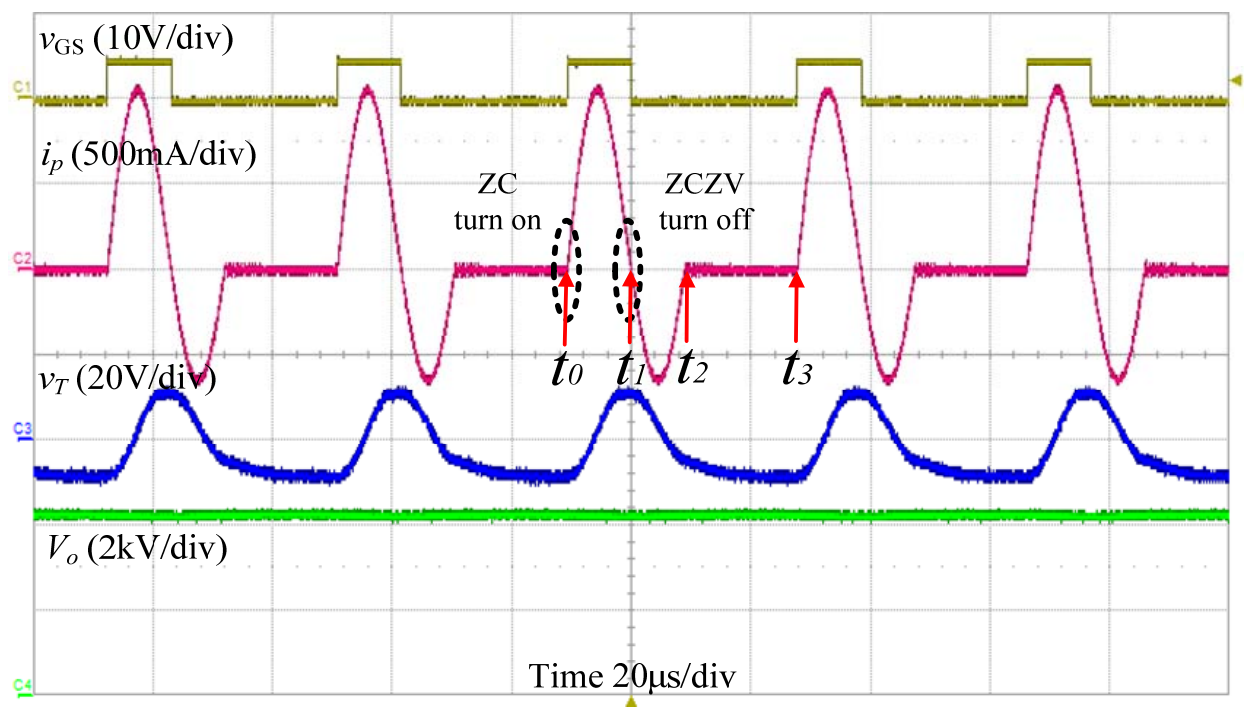

Fig. 4.13 Waveforms when the MOSFET is turned off at time $t_{1}, v_{\mathrm{GS}}$ : MOSFET gating signal, $i_{p}$ : primary side current, $v_{T}$ : lumped primary side voltage, $V_{o}$ : output voltage.

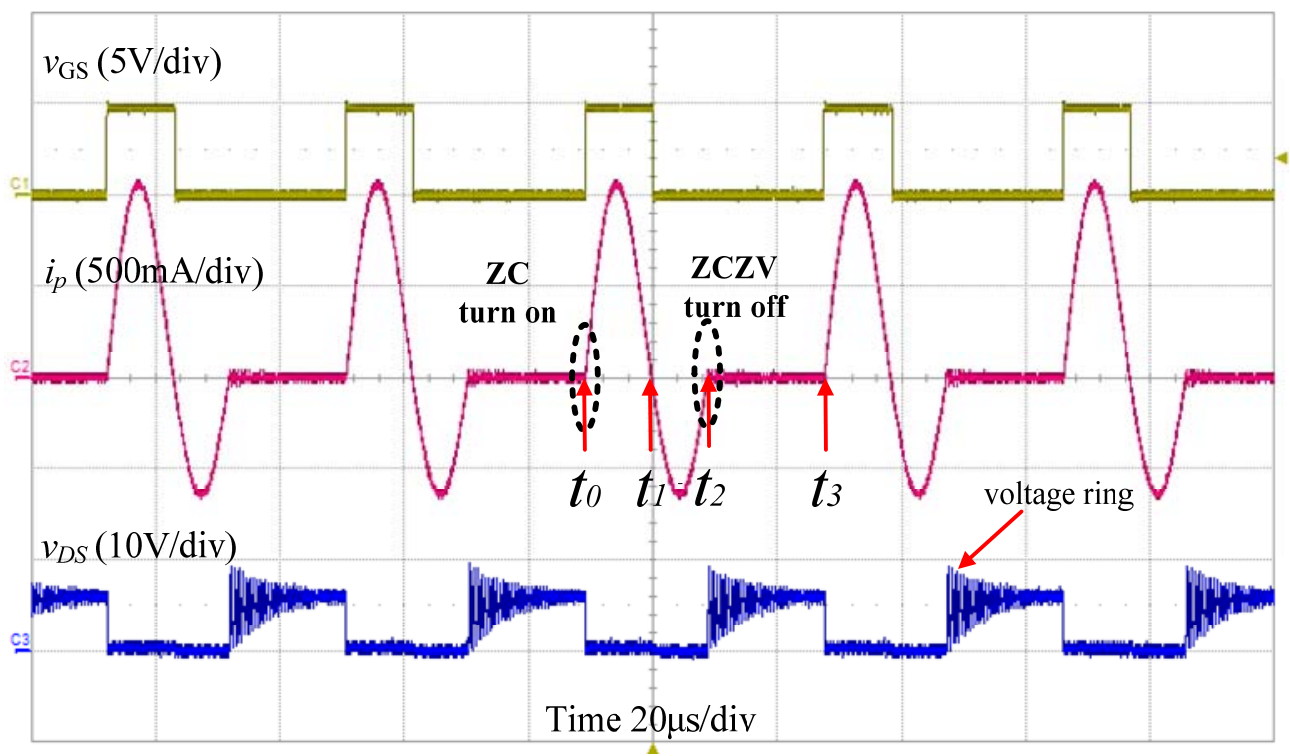

Fig. 4.14 Waveforms when the MOSFET is turned off at time $t_{1}$. $v_{\mathrm{GS}}$ : MOSFET gating signal, $i_{p}$ : primary side current, $v_{\mathrm{DS}}$ : drain-source voltage of MOSFET.

In Fig. 4.13, the load is $100 \mathrm{M} \Omega$, and the measured output voltage is $4.265 \mathrm{kV}$ from a $5 \mathrm{~V}$ input voltage. The output voltage is high enough as compared to the voltage requirements of PPT igniter in [21] $(1.5 \mathrm{kV})$ and [13] $(2.7 \mathrm{kV})$. The primary side current waveform $i_{p}$ is approximately sinusoidal during the time interval $t_{0}-t_{2}$. The series 
resonant inductor $L_{\text {in }}$ is operated with DCM because of Mode 3 during the time interval $t_{2}-t_{3}$. The switch can be turned on with zero-current (ZC) at time $t_{0}$, and off at time $t_{1}$ with zero-current zero-voltage (ZCZV). The waveform of $v_{T}$ during interval $t_{0}-t_{2}$ is also approximately sinusoidal with a resonant angular frequency of $\beta_{1}$. The voltage $v_{T}$ changes slowly during time interval $t_{2}-t_{3}$ because of large resonant angular frequency $\beta_{2}$ for Mode 3.

The drain-source voltage $v_{\mathrm{DS}}$ of MOSFET without any snubber circuit is also measured and shown in Fig. 4.14. The maximum $v_{\mathrm{DS}}$ is $19.4 \mathrm{~V}$. Due to soft-switching, no voltage spikes are observed. However, the output capacitor of MOSFET results in ringing at the start of Mode 3.

As discussed in Section 4.2.4, the proposed topology can turn off the switch with ZCZV anytime between $t_{1}$ and $t_{2}$. To verify this, an experiment has been performed when the MOSFET is turned off at time $t_{2}$. In Figs. 4.15 and 4.16 , the switching frequency is $26 \mathrm{kHz}\left(f_{n}=0.48\right)$, and the switch is turned off at $t_{2}$. The corresponding switching cycle is $t_{2} / T$ is $51.3 \%$. It is observed that ZCZV can also be obtained by turning off the switch at time $t_{2}$. With a load of $100 \mathrm{M} \Omega$, the experimental output voltage is $3.967 \mathrm{kV}$, and the delivered power is $157.3 \mathrm{~mW}$. The measured voltage gain is slightly smaller than when the switch is turned off at time $t_{1}$. The reason will be explained in the study of Fig. 4.17. 


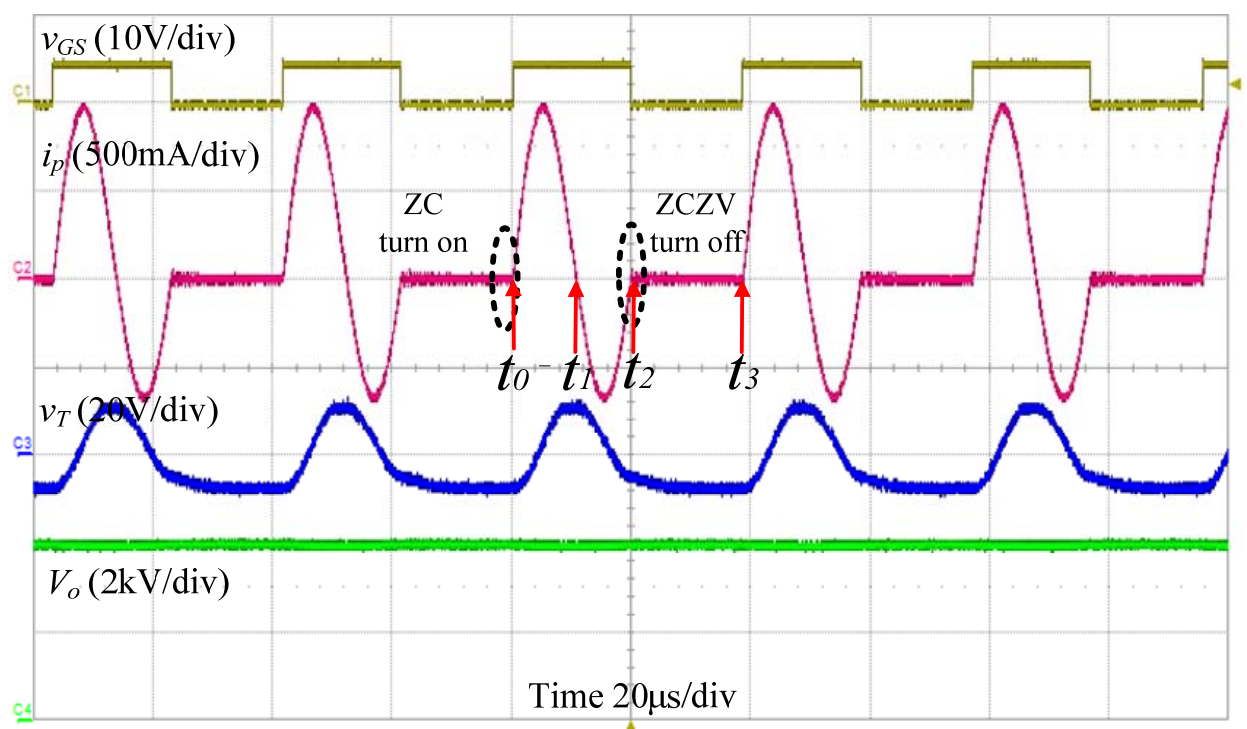

Fig. 4.15 Waveforms when the MOSFET is turned off at time $t_{2}$. $v_{\mathrm{GS}}$ : MOSFET gating signal, $i_{p}$ : primary side current, $v_{T}$ : lumped primary side voltage, $V_{o}$ : output voltage.

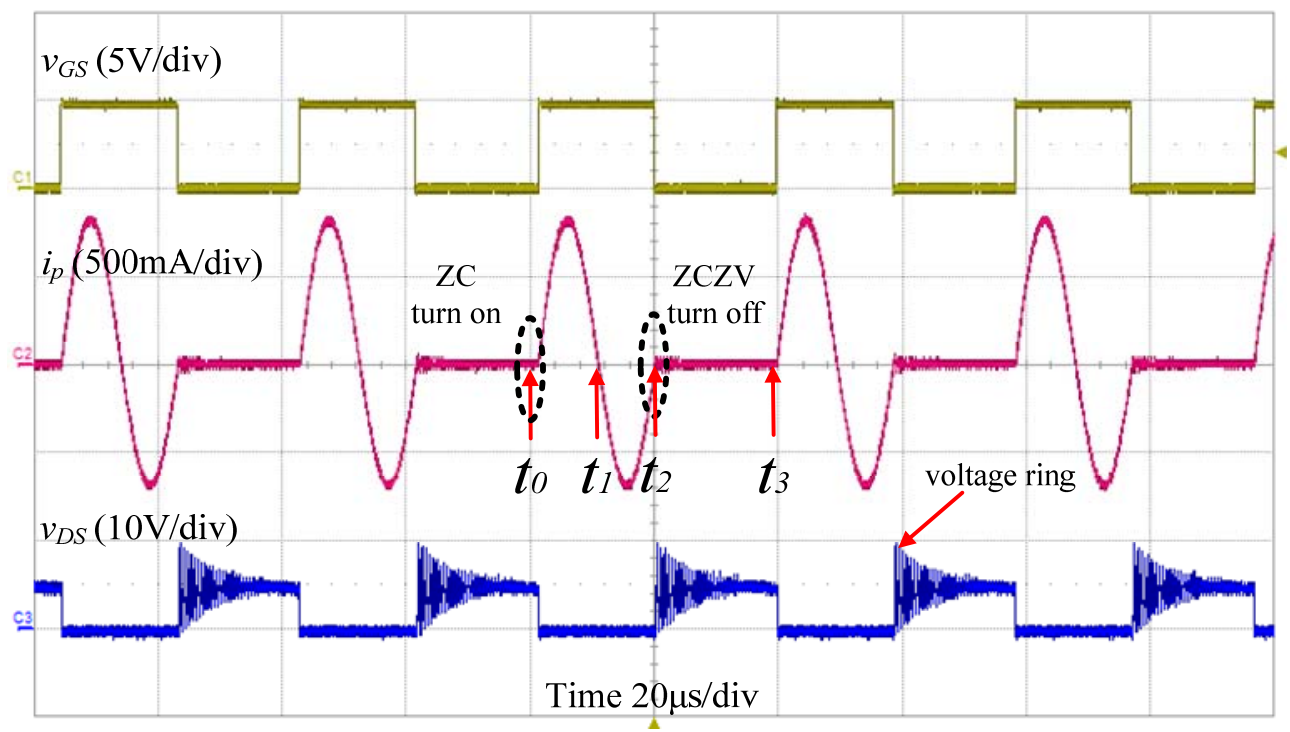

Fig. 4.16 Waveforms when the MOSFET is turned off at time $t_{2}$. $v_{\mathrm{GS}}$ : MOSFET gating signal, $i_{p}$ : primary side current, $v_{\mathrm{DS}}$ : drain-source voltage of MOSFET.

To validate the voltage regulation at light loads, simulation and experiment have been performed by changing the switching frequency. Fig. 4.17 shows the output voltage under various switching frequencies with a load of $100 \mathrm{M} \Omega$. The figure presents the 
theoretical, simulation and experimental results. The simulation study uses the circuit in Fig. 4.1 assuming that all the components are ideal.

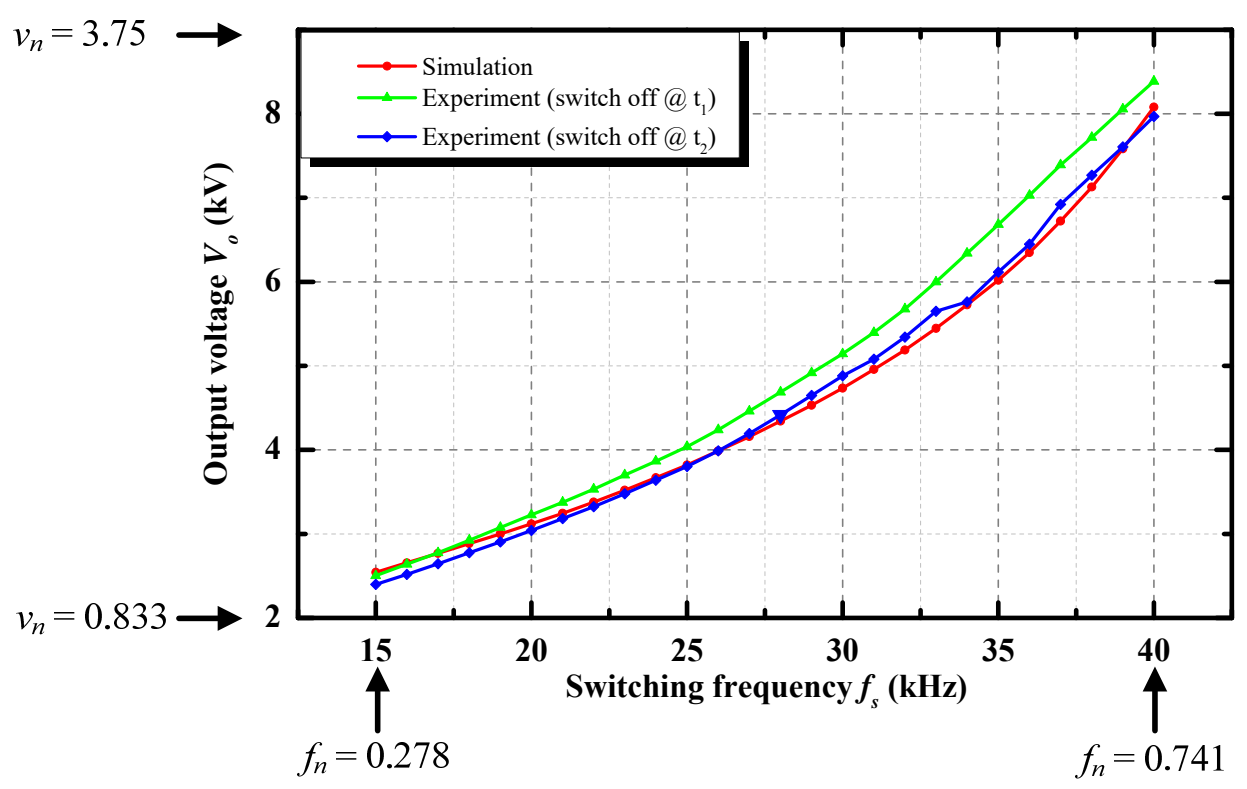

Fig. 4.17 Output voltage $V_{o}$ versus switching frequency $f_{s}$ by simulation and experiment.

Fig. 4.17 shows the experimental output voltage when the MOSFET is turned off both at $t_{1}$ and $t_{2}$. It is observed that the output voltage is slightly higher if the MOSFET is turned off at time $t_{1}$. This is caused by the non-ideality of MOSFET and freewheeling diode. In practice, the voltage applied to the resonant tank is the input voltage $V_{\text {in }}$ plus drain-source voltage of MOSFET during time interval $t_{1}-t_{2}$. If the switch is turned off at time $t_{1}$, the drain-source voltage is the threshold voltage $V_{\mathrm{SD}}$ of freewheeling diode plus the product of primary side current $i_{p}$ and turn-on resistance $R \mathrm{SD}$ of freewheeling diode. If the MOSFET is turned off at time $t_{2}$, the drain-source voltage is the product of primary side current $i_{p}$ and turn-on resistance $R_{\text {on }}$ of MOSFET. Based on the parameters of MOSFET in Table 4.2, the drain-source voltage of MOSFET during $t_{1}-t_{2}$ is larger if the switch is turned off at time $t_{1}$. Hence, the corresponding output voltage is higher. 
The full load of the proposed topology has been investigated by calculation, and the results are shown in Fig. 4.18. As discussed in Section 4.2.4, the ZCS operation can be realized because the current $i_{p}$ has the capability of reversing back to the input power supply. With the load power increasing, the reversing energy becomes less. This means time interval $t_{1}-t_{2}$ shown in Fig. 4.5 becomes shorter. When the time interval turns zero, $i_{p}$ cannot be reversed, and the maximum output power is generated. Consequently, the full load can be obtained by numerical approach by reducing the load resistor until $t_{1}=$ t2. It can be observed that the maximum delivered power is about $3.3 \mathrm{~W}$ at $40 \mathrm{kHz}$. Even though the proposed topology delivers low power, the energy is enough for a PPT igniter [14].

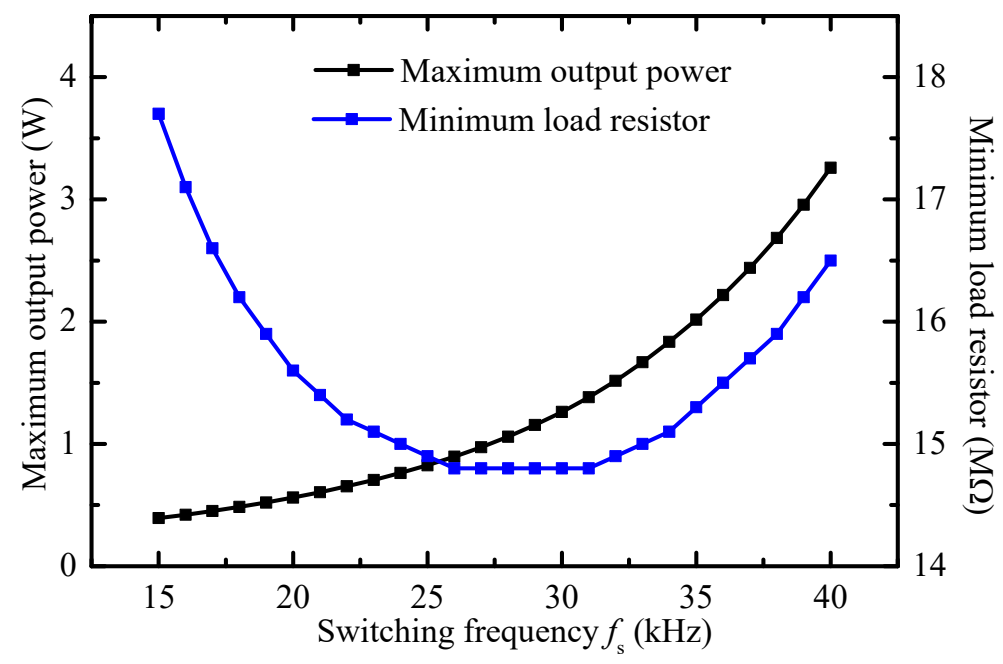

Fig. 4.18 Full load of the proposed topology versus switching frequency.

In the experiment, a resistor of $12 \mathrm{M} \Omega$ is the nearly minimum load resistor when the switching frequency is $26 \mathrm{kHz}$. Fig. 4.19 shows the experimental waveforms. With an increase in output power, smaller amount of energy flows back to the input power supply. It is observed that the reverse current and time interval $t_{1}-t_{2}$ are almost zero. Moreover, 
the waveforms have slight distortion. This is because a higher output power makes a larger voltage drop across the capacitors in the voltage multiplier. Hence, the turn-on time of diodes is longer, and the voltage $v_{T}$ is clamped. The measured output voltage $V_{o}$ is $3.603 \mathrm{kV}$, and delivered power is $1.082 \mathrm{~W}$, while the calculated maximum output power is $0.93 \mathrm{~W}$. The $7.3 \%$ error validates the theoretical analysis.

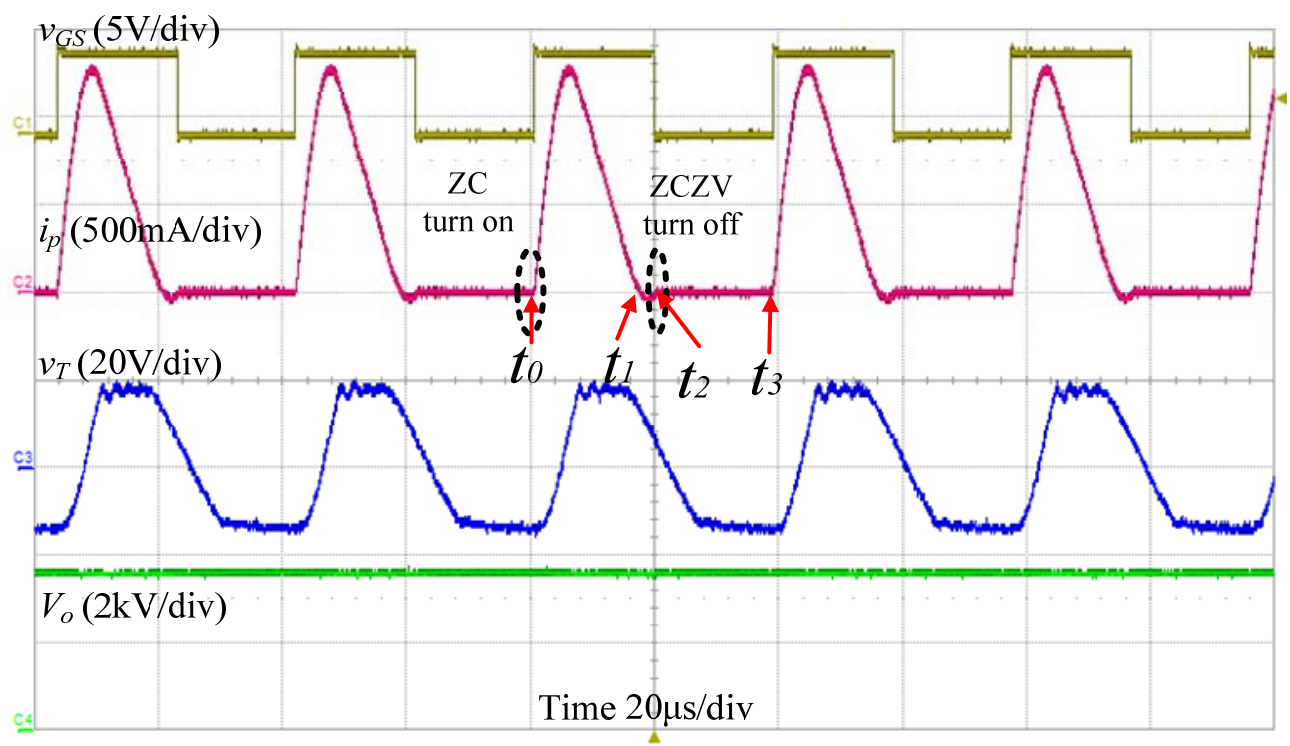

Fig. 4.19 Experimental waveforms with a load of $12 \mathrm{M} \Omega$. $v_{\mathrm{Gs}}$ : MOSFET gating signal, $i_{p}$ : primary side current, $v_{T}$ : lumped primary side voltage, $V_{o}$ : output voltage.

Experiment has also been conducted in vacuum environment to study the plasma generation. The prototype circuit for the igniter is integrated in the PPT system shown in Fig. 4.20(a). The experimental waveforms are shown in Fig. 4.21. The pulse frequency is set to $1 \mathrm{~Hz}$. When the firing control signal is high, the capacitor is charged to $1.7 \mathrm{kV}$ for the Teflon propellant ionization. When the firing control signal is low, the igniter circuit generates an arc if the output voltage is higher than $3.2 \mathrm{kV}$. It is observed that the prototype circuit is able to trigger the energy stored in the capacitor to release consistently. The generated plasma in the vacuum chamber is shown in Fig. 4.20(b). As discussed in Section 4.2.2, a more output power leads to a larger voltage drop. The 
minimum measured voltage in Fig. 4.21 is $3.2 \mathrm{kV}$. By (4.3), the equivalent load is 55.7 $\mathrm{M} \Omega$. The calculated peak power level is $183.8 \mathrm{~mW}$.

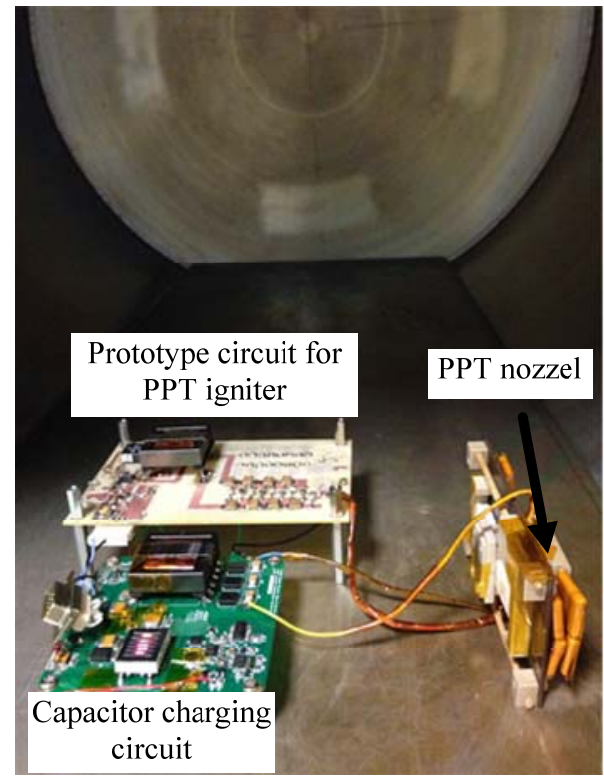

(a)

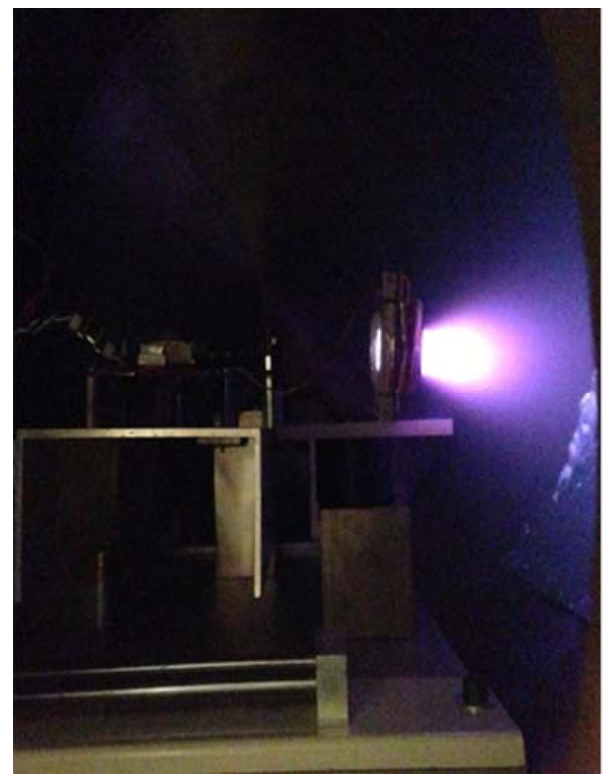

(b)

Fig. 4.20 (a) PPT system integration, and (b) plasma generation in vacuum chamber.

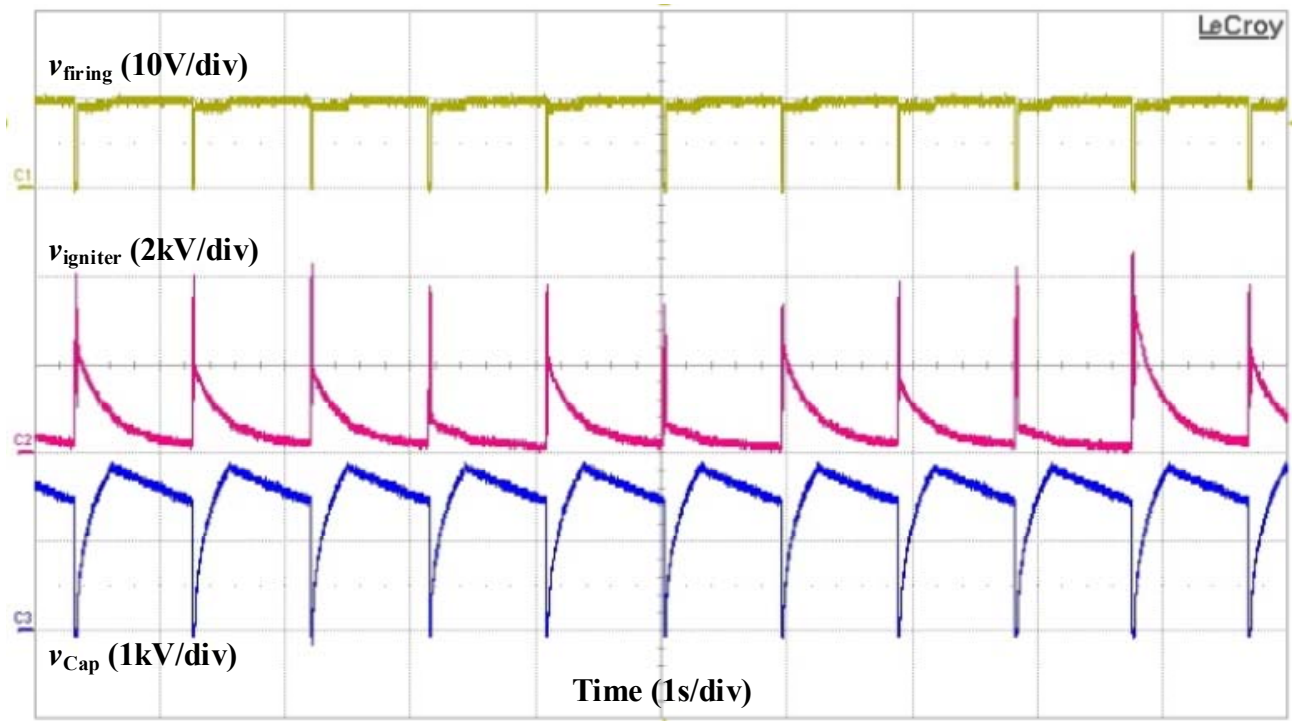

Fig. 4.21 Experimental waveforms in vacuum environment. $v_{\text {firing }}$ : firing control, $v_{\text {igniter }}$ : voltage of PPT igniter, $v_{\text {Cap }}$ : voltage of PPT capacitor bank. 


\subsection{Comparisons with Existing Resonant Converters}

In this section, the comparisons of proposed topology with existing resonant converters are presented. The results are summarized in Table 4.3.

\subsubsection{Number of Switches and Switching Control}

The proposed resonant tank in Fig. 4.1 has a different circuit arrangement from other resonant tanks shown in Fig. 2.5.

As summarized in Table 4.3, the proposed resonant tank uses only one switch as compared to other resonant tanks that use 2 or 4 switches. Besides cost and space saving, the use of a single switch eases the switching control without a dead-time, and improves the circuit reliability.

\subsubsection{Soft-switching and Range of Switching Frequency}

For series resonant converter (SRC), parallel resonant converter (PRC) and LCC resonant converter (LCC-RC), they are operated in discontinuous-current-mode (DCM) to achieve ZCS and reduce switch conduction loss. To realize DCM by a half- or fullbridge circuit, the normalized switching frequency $f_{n}$ should be less than 0.5 due to the switch bridge implementation $[55,59,70]$.

For LLC resonant converter (LLC-RC), it is operated in ZVS region, and the range of switching frequency is given in Table 4.3 [71].

For the proposed topology, it is operated with DCM to realize ZCS. With the advantage of a single switch implementation, the normalized switching frequency $f_{n}$ can be higher than 0.5 , wider than other resonant converters. 
Table 4.3 Comparisons of proposed topology with existing resonant converters

\begin{tabular}{|c|c|c|c|c|c|c|c|}
\hline $\begin{array}{l}\text { Resonant } \\
\text { converter }\end{array}$ & $\begin{array}{l}\text { Number } \\
\text { of } \\
\text { switches }\end{array}$ & $\begin{array}{l}\text { Dead- } \\
\text { time } \\
\text { control }\end{array}$ & $\begin{array}{l}\text { Soft- } \\
\text { switching }\end{array}$ & $\begin{array}{l}\text { Normalized } \\
\text { switching } \\
\text { frequency }\left(f_{n}\right)\end{array}$ & Normalized voltage gain $\left(v_{n}\right)$ & $\begin{array}{l}\text { Theoretical maximum } \\
\text { normalized voltage gain }\left(v_{n, \max }\right)\end{array}$ & $\begin{array}{l}\text { Voltage stress on } \\
\text { switches }\end{array}$ \\
\hline $\mathrm{SRC}$ & 4 or 2 & Yes & ZCS & $<0.5$ & $\frac{1}{\sqrt{1+Q^{2}\left(f_{n}-1 / f_{n}\right)^{2}}}$ & $\begin{array}{c}1 \\
\text { Open load }(\mathrm{Q}=0) \text {. }\end{array}$ & $V_{\text {in }}$ \\
\hline PRC & 4 or 2 & Yes & ZCS & $<0.5$ & $\frac{1}{\sqrt{\left(1-f_{n}^{2}\right)^{2}+\left(f_{n} / Q\right)^{2}}}$ & $\begin{array}{c}1.33 \\
\text { Open load }(\mathrm{Q}=\infty) ; f_{n}=0.5\end{array}$ & $V_{\text {in }}$ \\
\hline LCC-RC & 4 or 2 & Yes & $\mathrm{ZCS}$ & $<0.5$ & $\frac{1}{\sqrt{\left[1+n_{c}-n_{c} f_{n}^{2}\right]^{2}+\left[Q\left(f_{n}-f_{n}^{-1}\right)\right]^{2}}}$ & $\begin{array}{c}1 /\left(1+0.75 n_{c}\right) \\
\text { Open load }(\mathrm{Q}=0) ; f_{n}=0.5\end{array}$ & $V_{\text {in }}$ \\
\hline LLC-RC & 4 or 2 & Yes & ZVS & $\left(\frac{1}{\sqrt{m}}, 1\right)^{* *}$ & $\frac{1}{\sqrt{\left[\frac{m}{m-1}-\frac{f_{n}^{-2}}{m-1}\right]^{2}+\left[Q\left(f_{n}-f_{n}^{-1}\right)\right]^{2}}}$ & $\begin{array}{c}\infty \\
\text { Open } \operatorname{load}(\mathrm{Q}=0) ; f_{n}=1 / \sqrt{m}\end{array}$ & $V_{\text {in }}$ \\
\hline $\begin{array}{l}\text { Proposed } \\
\text { topology }\end{array}$ & 1 & No & $\mathrm{ZCS}$ & $<1$ & Numerical calculation & - & $V_{\mathrm{in}}+v_{\mathrm{T}, \max }$ \\
\hline
\end{tabular}

$Q$ : Load quality factor of resonant converters.

* $\quad n_{c}=C_{p} / C_{r}\left(C_{p}\right.$ and $C_{r}$ are shown in Fig. 2.5(c)).

$* * \quad m=\left(L_{m}+L_{r}\right) / L_{r}\left(L_{m}\right.$ and $L_{r}$ are shown in Fig. $\left.2.5(\mathrm{~d})\right)$. 


\subsubsection{Normalized Voltage Gain}

The formulae of normalized voltage gain $v_{n}$ listed in Table 4.3 are derived by the first harmonic approach (FHA). The results of the proposed topology are calculated numerically using the components listed in Table 4.2. Fig. 4.22 compares their results with open load when the maximum voltage gain is achieved. It is observed that the normalized voltage gain of SRC is 1 without capability of output voltage regulation. The normalized voltage gain of PRC is less than 1.33, while LCC-RC is less than 1 . Their boost capabilities are limited by the constraint $f_{n}<0.5$. For the proposed topology, it can achieve a normalized voltage gain of 6.2 at $f_{n}=0.87$, exhibiting a better boost capability than PRC and LCC-RC operating with ZCS. The LLC-RC also exhibits good boost capability when working in the ZVS region. However, a higher voltage gain is achieved at a lower switching frequency. This results in a bigger size of magnetic components.

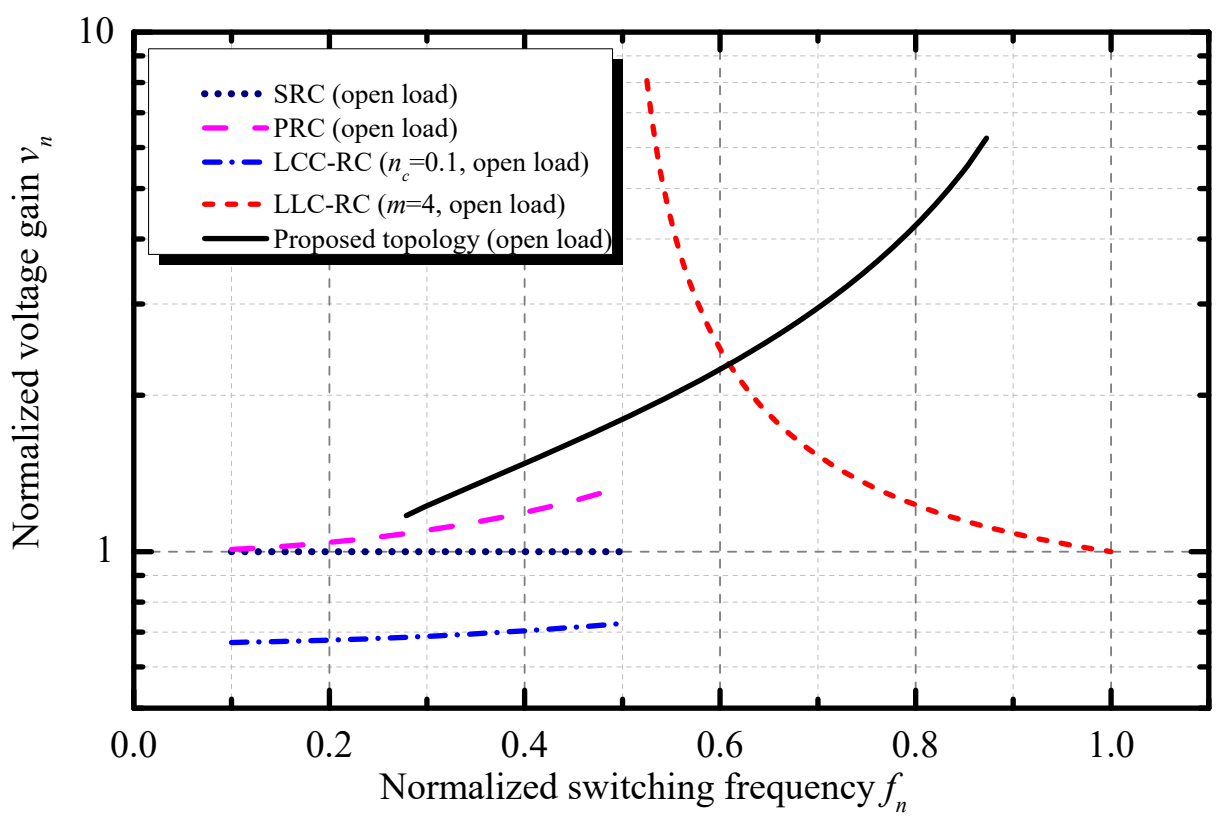

Fig. 4.22 Comparison on normalized voltage gain $v_{n}$. 


\subsubsection{Voltage Stress on Switches}

For a circuit using half- or full-bridge switches, the voltage stress on MOSFET is the

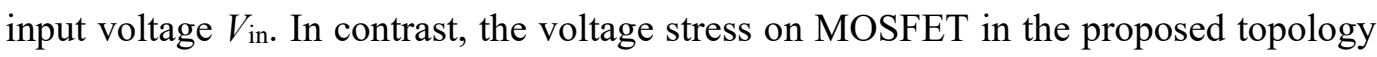
is $V_{\mathrm{in}}+v_{T, \text { max }}$ where $v_{T, \text { max }}$ is the maximum amplitude of voltage $v_{T}$. The voltage stress will be higher with the increase in voltage gain. This is the drawback of using a single switch.

\subsubsection{Current and Efficiency}

From Fig. 4.22, the LLC-RC and proposed topology have a better voltage gain compared to other topologies. Therefore, the comparisons on MOSFET current, switch conduction loss and efficiency are conducted between them. The study is performed by PSIM simulation with a load of $100 \mathrm{M} \Omega$. The proposed topology uses the circuit shown in Fig. 4.1 with the components listed in Table 4.2 as well as the MOSFET parameters. For LLC-RC, the same resonant frequency a half-bridge circuit are used. The LLC-RC generates the same output voltage as the proposed topology under the same switching frequency by adjusting $m$ defined in Table 4.3.

Fig. 4.23 shows the comparisons on the maximum and root-mean-square (RMS) current in MOSFET. Because of being used for low output power applications, the topologies do not suffer high current stress even though they are operated with discontinuous-current-mode (DCM). To obtain a higher voltage with the same switching frequency, magnetizing inductance $L_{m}$ in LLC-RC decreases. Hence, the decrease in overall impedance leads to a larger current when the output voltage is higher than $7 \mathrm{kV}$. It is also observed that the proposed topology has a higher current if the MOSFET is turned off at time $t_{1}$. This is because the corresponding output voltage is higher, which will be discussed further in the observation of Fig. 4.24. 


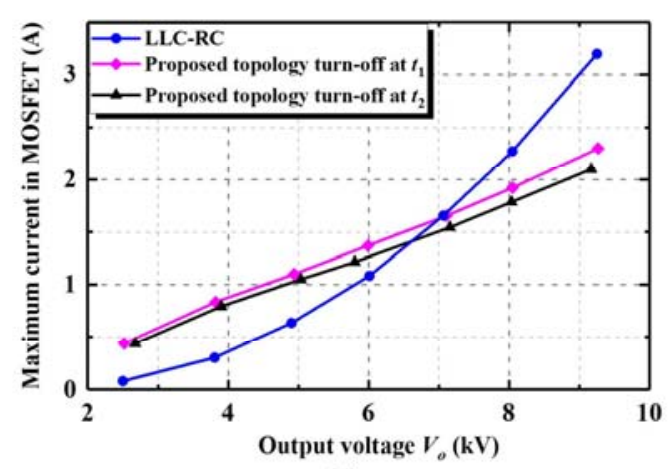

(a)

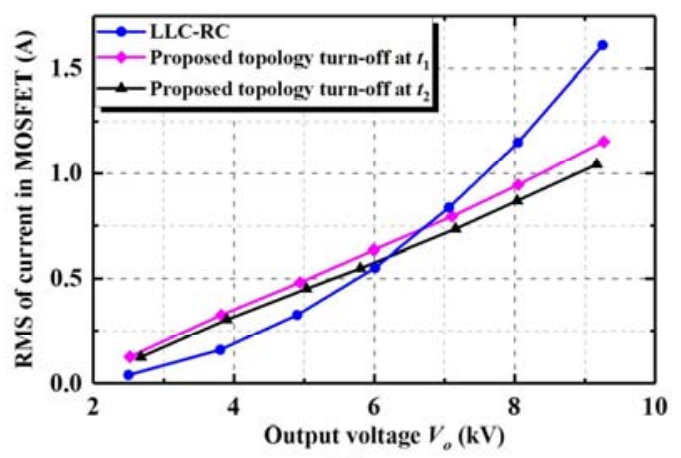

(b)

Fig. 4.23 Comparison on MOSFET current between LLC resonant converter and proposed topology: (a) maximum current; (b) root-mean-square (RMS) current.

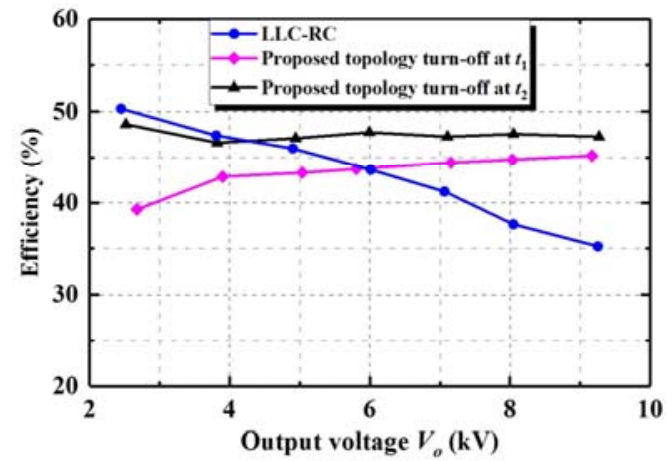

(a)

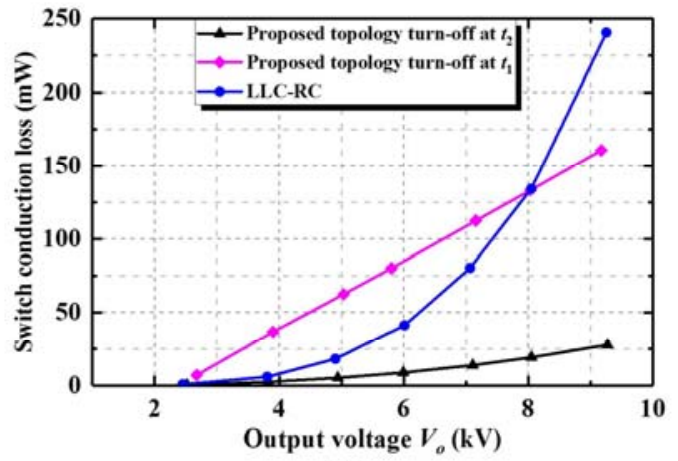

(b)

Fig. 4.24 Comparison on (a) efficiency, and (b) switch conduction loss between LLC resonant converter and proposed topology.

Fig. 4.24 shows comparisons on efficiency and switch conduction loss. It is observed that the efficiency of proposed topology is different with the turn-off time of MOSFET. This is explained as follows.

As shown in Fig. 4.5, the proposed topology can achieve turn-off with zero-current zero-voltage (ZCZV) anytime between $t_{1}$ and $t_{2}$. The switch conduction loss $W_{\text {loss_Mode2 }}$ is caused by the MOSFET and the freewheeling diode during $t_{1}-t_{2}$. It is determined by

$$
W_{\text {loss_Mode } 2}=\int_{t_{1}}^{t_{\text {off }}} i_{p}^{2} R_{o n} d t+\int_{t_{\text {off }}}^{t_{2}}\left(i_{p}^{2} R_{\mathrm{SD}}+V_{\mathrm{SD}} i_{p}\right) d t
$$


where $R_{\mathrm{on}}$ is the drain-source on-resistance of the MOSFET; $R_{\mathrm{SD}}$ and $V_{\mathrm{SD}}$ are the onresistance and threshold voltage of freewheeling diode, respectively. Refer to the MOSFET parameters listed in Table 4.2, there will be more conduction loss in Mode 2 caused by the freewheeling diode when the MOSFET is turned off at time $t_{1}$, resulting in lower efficiency as compared to the case when the MOSFET is turned off at time $t_{2}$. From Fig. 4.24, the efficiency of proposed topology is between $39.3 \%$ and $45.2 \%$ when the MOSFET is turned off at $t_{1}$. In contrast, the efficiency is between $46.5 \%$ and $48.6 \%$ when the MOSFET is turned off at $t$.

From Fig. 4.24(a), the best efficiency of LLC-RC is $50.3 \%$ when $m=21.6$ and $V_{o}=$ $2.45 \mathrm{kV}$. A higher output voltage can be obtained by a smaller $m$. For example, the output voltage is $9.25 \mathrm{kV}$ when $m=1.76$. However, the efficiency decreases simultaneously. This is because an LLC-RC behaves more like an SRC with the advantage of reduced circulating current when $m$ is larger. However, the output voltage regulation is poor under light load condition (load quality factor $\mathrm{Q} \approx 0$ ) [20].

When the MOSFET is turned off at $t_{2}$, the proposed topology behaves better than an LLC-RC with $m$ smaller than 3.79 in terms of efficiency. This is because the switch conduction loss is reduced due to the single switch implementation and ZCS operation. For example, the switch conduction loss of the proposed topology is $27.62 \mathrm{~mW}$ while that of LLC-RC is $240.48 \mathrm{~mW}$ when the output voltage is $9.2 \mathrm{kV}$. 


\subsection{Comparisons with Circuits Using a Single Switch}

\subsubsection{Comparisons with Flyback Circuit}

For a PPT igniter, Chapter 3 discusses a flyback circuit using hard-switching, while Chapter 4 proposes a single switch quasi-resonant converter using soft-switching. From the experimental results, both the proposed circuits can ignite the plasma generation in vacuum environment successfully. This section compares the two igniter circuits, and their characteristics are summarized in Table 4.4.

Table 4.4 Comparisons between flyback circuit and single switch quasi-resonant converter

\begin{tabular}{l|l|l|l|l}
\hline \hline Topology & $\begin{array}{l}\text { Number of } \\
\text { Components }\end{array}$ & $\begin{array}{l}\text { Switching } \\
\text { method }\end{array}$ & $\begin{array}{l}\text { Output voltage } \\
\text { regulation }\end{array}$ & Output power \\
\hline Flyback circuit & $\begin{array}{l}1 \times \text { Transformer, } \\
1 \times \text { MOSFET, } \\
\text { Control circuit. }\end{array}$ & $\begin{array}{l}\text { Hard- } \\
\text { switching }\end{array}$ & $\begin{array}{l}\text { Charging current } \\
\text { limit } I_{\text {lim }}\end{array}$ & $<2.1 \mathrm{~mW}$ \\
\hline $\begin{array}{l}\text { Single switch } \\
\text { quasi-resonant } \\
\text { DC-DC } \\
\text { converter }\end{array}$ & $\begin{array}{l}1 \times \text { Transformer, } \\
1 \times \text { MOSFET, } \\
1 \times \text { Resonant capacitor, } \\
1 \times \text { Resonant inductor, } \\
1 \times m \text {-stage VM, } \\
\text { Control circuit. }\end{array}$ & $\begin{array}{l}\text { Soft- } \\
\text { switching }\end{array}$ & $\begin{array}{l}\text { Switching } \\
\text { frequency } f_{\mathrm{s}}\end{array}$ & $<3.3 \mathrm{~W}$ \\
\hline \hline
\end{tabular}

For the flyback circuit, a smaller number of components are used. The major advantage is that it does not require a high voltage capacitor. This can improve the reliability by $13.4 \%$. However, the hard-switching approach requires a MOSFET with a higher drain-source breakdown voltage. Hence, the MOSFET selection should be considered carefully. In contrast, the single switch quasi-resonant DC-DC converter uses the soft-switching technique. Due to the quasi-resonant operation, the MOSFET can be turned on with zero-current (ZC), and off with zero-current zero-voltage (ZCZV) to eliminate the voltage spikes. Hence, the voltage stress on the MOSFET is smaller.

To guarantee the igniter can operate in space, the output voltage should be adjustable. For the flyback circuit, the output voltage is regulated simply by the changing the 
charging current limit $I_{\text {lim, }}$ while the single switch quasi-resonant DC-DC converter regulates the output voltage by changing the switching frequency.

For the flyback circuit, the energy is only stored in the transformer magnetizing inductance. Hence, the output power is smaller. From Table 3.2, the maximum output power of the prototype is $2.085 \mathrm{~mW}$ if the charging current limit $I_{\mathrm{lim}}$ uses the saturation current of the ferrite core, and the pulse frequency is set to $1 \mathrm{~Hz}$. The single switch quasiresonant DC-DC converter can deliver more power. The amount of power depends on the PPT design. In [14], a power of $0.01 \mathrm{~W}$ is required by the PPT igniter. In this case, the flyback circuit is not applicable.

From Fig. 3.10, the flyback circuit has the best efficiency of $38.1 \%$. In contrast, the single switch quasi-resonant DC-DC converter has the lowest efficiency of 39.3\% from Fig. 4.24. Hence, the topology using soft-switching behaves better in terms of efficiency.

\subsubsection{Comparisons with Quasi-resonant Flyback Converter}

Both the quasi-resonant flyback converter in [77-79] and proposed topology in Chapter 4 utilize a single switch to reduce the number of components. However, they have several differences. The corresponding advantages and disadvantages are discussed as follows:

First, the proposed topology has requirements on the values of resonant components to obtain ZCS from Fig. 4.8. Hence, an inductor and a capacitor are added. This increases the cost and complexity compared to a quasi-flyback converter without extra components.

Second, the input current of a quasi-flyback converter increases linearly when the power switch is turned on. In contrast, the input current of the proposed topology is quasi-sinusoidal. Hence, the risk of electromagnetic interference (EMI) issue is reduced. 
Third, the soft-switching approaches are different. A quasi-flyback converter uses valley-voltage-switching. As the power switch is not turned on with zero-voltage, nor off with zero-current, switching loss exists. For the proposed topology, the power switch is turned on with zero-current, and off with zero-current zero-voltage. Hence, the switching loss is eliminated.

\subsection{Summary}

This chapter proposes a single switch quasi-resonant DC-DC converter for the igniter of pulsed plasma thruster (PPT). The resonant tank makes use of the leakage inductance, magnetizing inductance and winding capacitance of transformer for the basic circuit operation. This reduces the adverse effect caused by the transformer nonideality.

As the energy flow path of shunt capacitor can be provided by shunt inductor, the resonant tank can be driven by a single switch to operate in quasi-resonant state. The soft-switching of proposed circuit reduces voltage spikes, switching noise and switching loss. Using only a single switch makes it easier to build a redundant circuit to improve the system reliability. Moreover, the switching control can be realized without a deadtime. As the switching frequency can be extended to higher than half of the resonant frequency, it has a better voltage boost capability than other resonant converters operating with zero-current-switching (ZCS). Its output voltage is adjustable by changing the switching frequency. Successful plasma generation triggered by the prototype circuit has been demonstrated in vacuum environment. 


\section{Chapter 5}

\section{Quasi-resonant Flyback Converter for Capacitor Charging}

\subsection{Introduction}

As introduced in Chapter 1, the pulsed plasma thruster (PPT) needs a capacitor charging circuit. When the igniter works, the energy stored in capacitor is released to ablate and ionize the Teflon propellant into plasma. Constrained by the power budget of nano-satellite, the maximum energy of one release is set to $1.5 \mathrm{~J}$ as listed in Table 2.1.

Chapter 2 has reviewed topologies suitable for capacitor charging. Among these topologies, the conventional flyback and forward converters shown in Fig. 2.7 use hardswitching approaches resulting in voltage spikes and switching loss. To overcome the drawbacks, a soft-switching technique is preferred to improve the efficiency and reliability. For the resonant converters shown in Fig. 2.5, a switch bridge is required. Hence the switching control would consider a dead-time to avoid the short circuit of the same leg. Moreover, it is complex to implement soft-switching since the capacitor behaves as a short circuit in the beginning, and an almost open circuit when the output voltage approaches the maximum.

The quasi-resonant flyback converter inherits the advantages of conventional flyback converter and resonant converter, such as small number of components, softswitching, voltage boost capability, etc. The reported studies have used valley-voltageswitching (VVS). As presented in Section 2.2.3, the VVS operation in Fig. 2.8(a) has a limited output voltage, while that in Fig. 2.8(b) leads to energy loss by freewheeling 
diode. Moreover, the reviewed literatures have only investigated the quasi-resonant operation in steady state. To overcome the drawbacks and use quasi-resonance for capacitor charging, new soft-switching approaches are proposed in this chapter.

In this chapter, a quasi-resonant flyback converter shown in Fig. 5.1 is proposed to charge capacitor. To achieve a desired output voltage, multiple identical secondary windings (two secondary windings are demonstrated in Fig. 5.1) can be connected in series. Unlike other quasi-resonant only using parasitic capacitance, a capacitor $C_{\text {in }}$ is added to suppress the voltage stress on semiconductor switch $S_{1}$. Capacitor $C_{\text {in }}$ is lumped into resonant capacitance $C_{r}$ in Fig. 5.3 together with other parasitic capacitances including switch output capacitance $C_{\text {oss }}$ and transformer winding capacitance $C_{w}$. According to operating conditions, the switching control can be realized by two ways. One is zero-voltage-switching (ZVS) throughout the charging process, while the other is hybrid switching which introduces valley-voltage-switching (VVS) in the first stage, and ZVS in the remaining charging process. ZVS and VVS can be obtained due to the quasi-resonance between transformer magnetizing inductance $L_{m}$ and lumped resonant capacitance $C_{r}$. Hence, the switching-on loss and current spikes can be minimized. The quasi-resonance makes the diode $\left(D_{s 1}\right.$ or $\left.D_{s 2}\right)$ in the secondary side turn on with zerovoltage, and off with zero-current. Hence, the turn-on overshoot voltage and recovery loss can be mitigated. Since the diode in the secondary side does not conduct instantly when power switch $S_{1}$ is turned off. Consequently, the switching-off loss is reduced without voltage reflected from the output voltage. The energy stored in leakage inductance $L_{\mathrm{lk}}$ cannot be dissipated by freewheeling diode $D_{1}$, and may be recycled to the load capacitor. This helps improve the charging efficiency. The reverse input current $i_{\text {in }}$ in the beginning of charging cycle demagnetizes the transformer to avoid ferrite core saturation. 
In this chapter, the details of switching control for capacitor charging will be presented. The operating modes and the mathematical analysis are first described. This is then followed by the simulation and experimental studies. Finally, the capacitor charging performance of quasi-resonant flyback converter is compared with a conventional flyback converter.

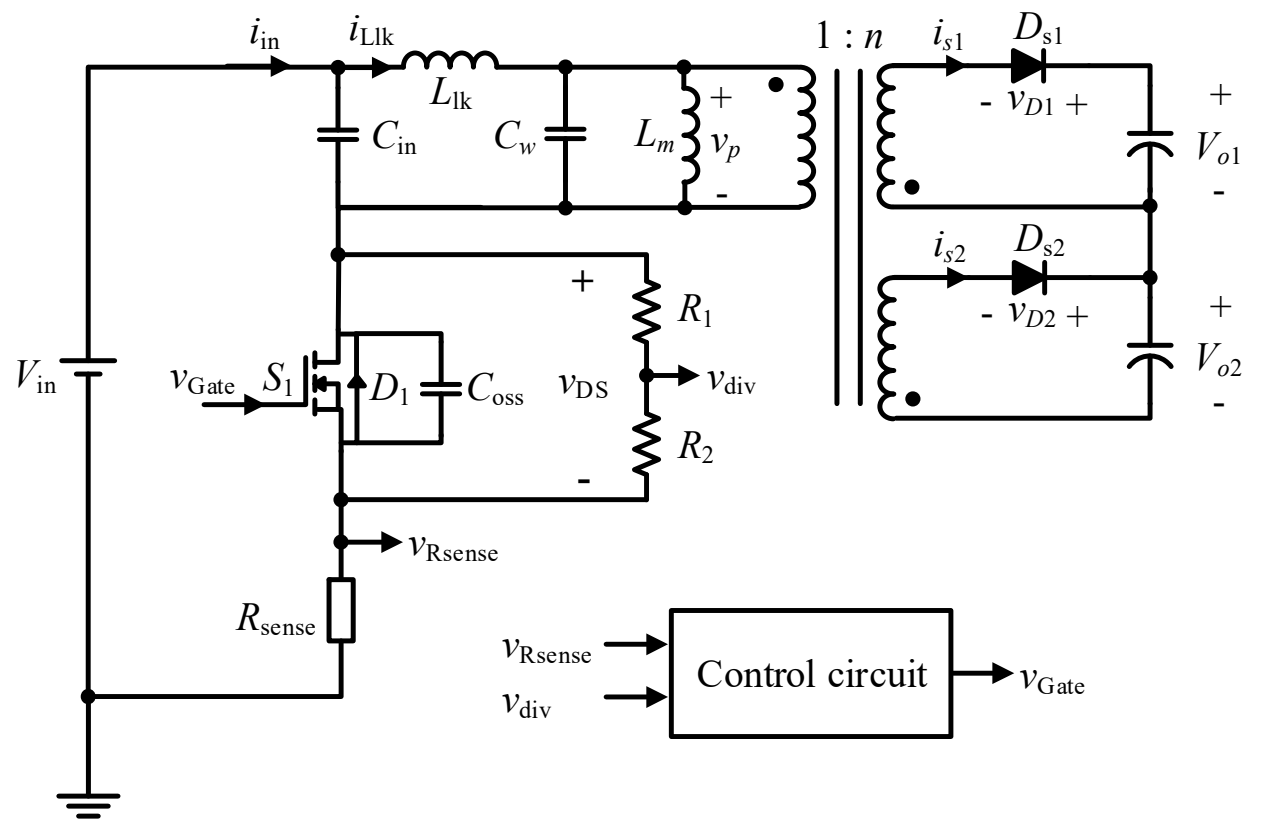

Fig. 5.1 Quasi-resonant flyback converter for capacitor charging.

\subsection{Principle of Operation}

For identical secondary windings, their behaviors are the same. Consequently, only one secondary winding is considered in this study. A metal-oxide-semiconductor fieldeffect transistor (MOSFET) with freewheeling diode $D_{1}$ is used as semiconductor switch $S_{1}$. An external capacitor $C_{\text {in }}$ is added in parallel with the primary side for soft-switching control. Capacitor $C_{\text {in, }}$ transformer winding capacitance $C_{w}$ and MOSFET output 
capacitance $C_{\text {oss }}$ are lumped to resonant capacitance $C_{r}$. Other components are assumed to be ideal.

\subsubsection{Quasi-resonant Operation}

The nominal operating waveforms of valley-voltage-switching (VVS) and zerovoltage-switching (ZVS) in the $k^{\text {th }}$ charging cycle are illustrated in Figs. 5.2(a) and (b), respectively. The quasi-resonant operation can be subdivided into four time intervals.

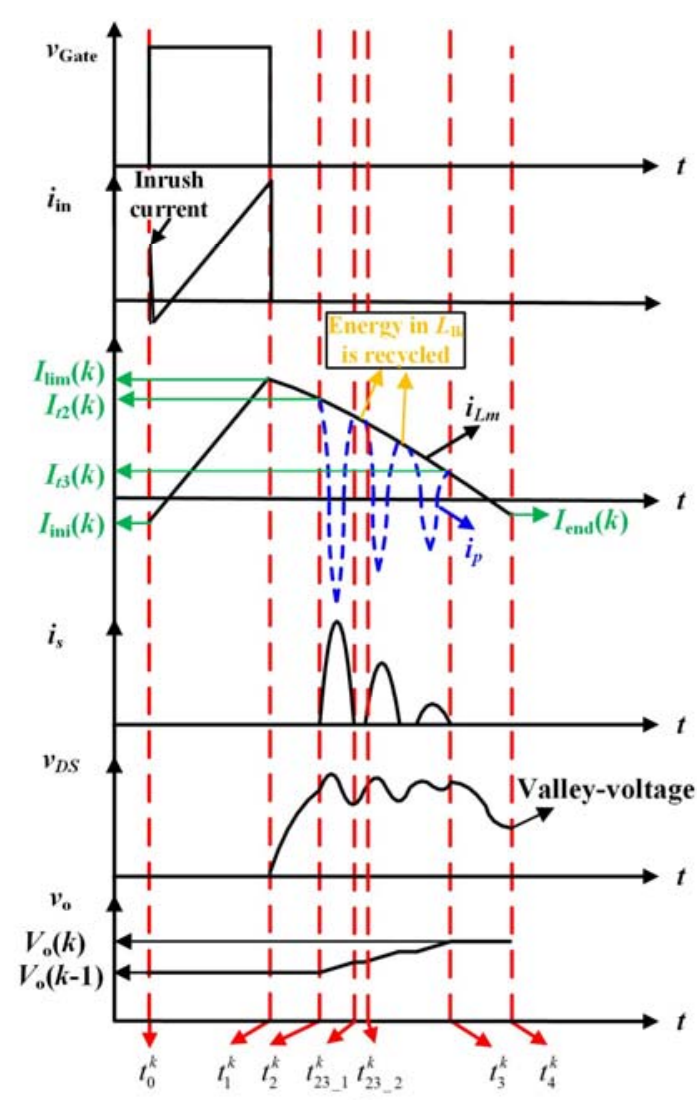

(a)

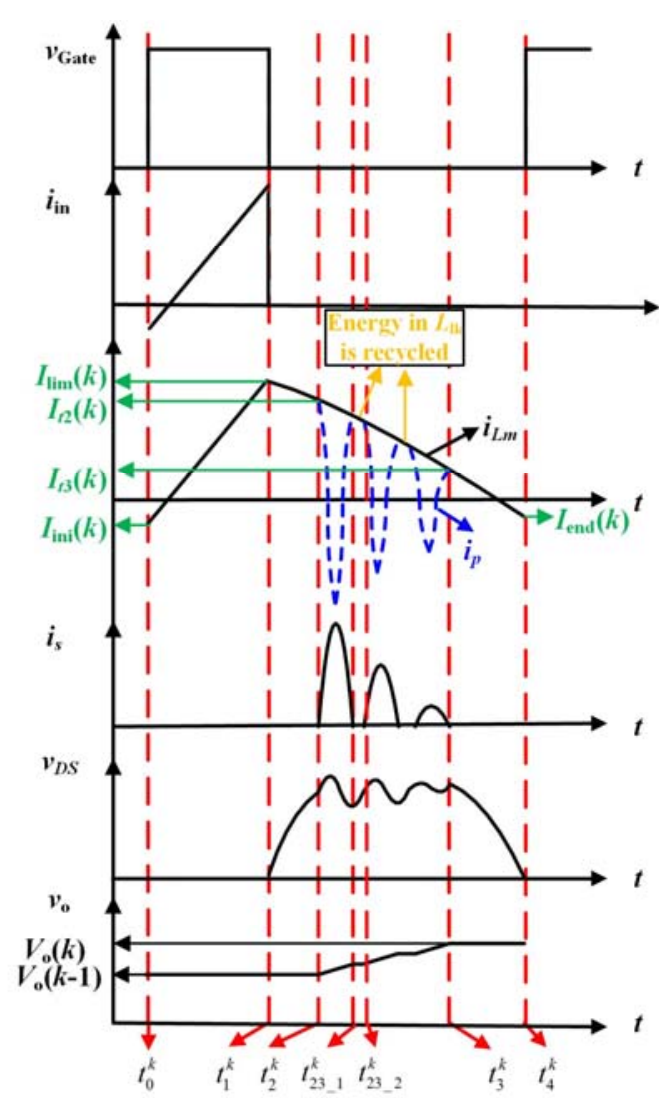

(b)

Fig. 5.2 Operating waveforms in the $k^{\text {th }}$ charging cycle of (a) VVS; (b) ZVS. $v_{\text {Gate }}$ : gating signal of

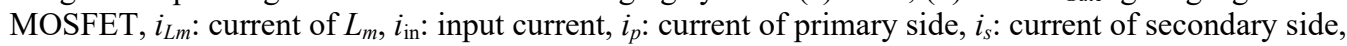
$v_{\mathrm{DS}}$ : drain-source voltage of MOSFET, $v_{o}$ : output voltage.

1) Mode $1\left[t_{0}^{k}-t_{1}^{k}\right]$ : Semiconductor switch $S_{1}$ (MOSFET) is turned on at time $t_{0}^{k}$ with an initial current $I_{\text {ini }}(k)$ which is equal to the ending current $I_{\text {end }}(k-1)$ of last charging 
cycle. The primary side of transformer is applied by the input voltage $V_{\text {in }}$, making the current in $L_{m}$ increase linearly. The turn-on inrush current is minimized by VVS while it can be eliminated by ZVS. Consequently, the circuit reliability can be improved with a reduced inrush current [120]. Due to the reverse winding polarity of flyback transformer, diode $D_{s}$ is blocked in this mode. The equivalent circuit of Mode 1 is shown in Fig. 5.3(a).

The primary side voltage $v_{p}^{k}$, magnetizing inductance current $i_{L_{m}}^{k}$ and primary side current $i_{p}^{k}$ are

$$
\left\{\begin{array}{l}
v_{p}^{k}(t)=a V_{\text {in }} \\
i_{L_{m}}^{k}(t)=i_{p}^{k}(t)=I_{\text {ini }}(k)+\frac{V_{\text {in }}}{L_{m}+L_{\mathrm{lk}}}\left(t-t_{0}^{k}\right)
\end{array}\right.
$$

where $a=\frac{L_{m}}{L_{m}+L_{\mathrm{lk}}}$.

The input current $i_{\text {in }}^{k}$ is equal to $i_{p}^{k}$. The output voltage remains $V_{o}(k-1)$ which is charged by the previous $k$ - 1 cycles. For the repetitive discharging in the PPT application, the output voltage is zero before the first charging cycle, i.e. $V_{o}(0)=0$. For the first charging cycle, the initial current is zero, i.e. $I_{\text {ini }}(1)=0$.

This time interval ends when the input current $i_{\text {in }}^{k}$ reaches charging current limit $I_{\lim }(k)$ at time $t_{1}^{k}$. The determination of $I_{\lim }(k)$ will be discussed in Section 5.2.2. The time duration of Mode 1 is $T_{1}^{k}$ determined by

$$
T_{1}^{k}=\frac{\left(L_{m}+L_{\mathrm{lk}}\right)\left[I_{\mathrm{lim}}(k)-I_{\mathrm{ini}}(k)\right]}{V_{\mathrm{in}}} .
$$




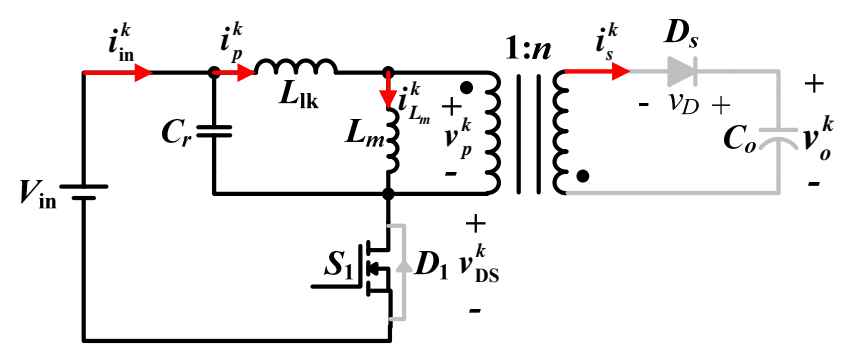

(a)

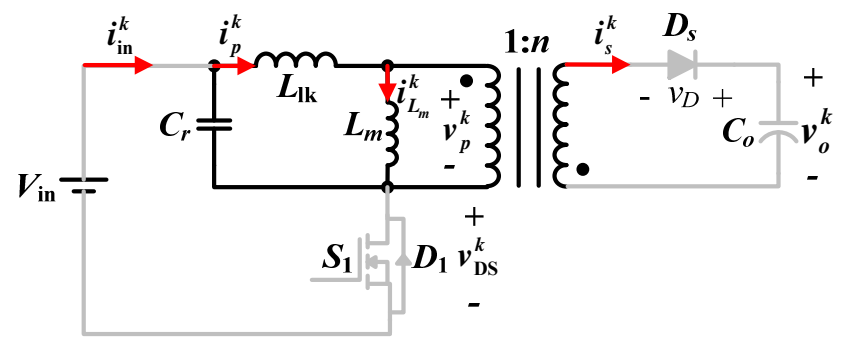

(b)

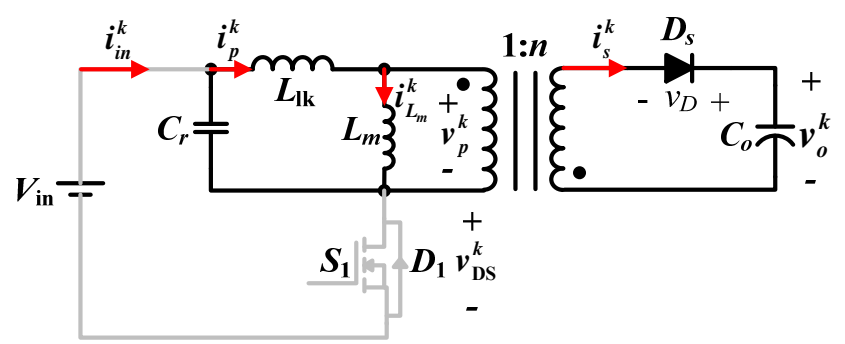

(c)

Fig. 5.3 Equivalent circuit for (a) Mode 1, (b) Modes 2 and 4, and (c) Mode 3.

2) Mode $2\left(t_{1}^{k}-t_{2}^{k}\right)$ : Once the MOSFET is turned off at time $t_{1}^{k}$, the quasi-resonance starts. When the amplitude of $v_{p}^{k}$ is smaller than $V_{o}(k-1) / n$, diode $D_{s}$ remains blocked, and load capacitor $C_{o}$ is not charged. Hence, the quasi-resonance occurs among $L_{m}, L_{\mathrm{k}}$ and $C_{r}$. The input current $i_{\text {in }}^{k}$ remains zero. The equivalent circuit of Mode 2 is shown in Fig. 5.3(b), and the resonant behavior can be described by the following second-order differential equation:

$$
\frac{d^{2} i_{p}^{k}}{d t^{2}}+\omega_{o 1}^{2} i_{p}^{k}=0
$$


By the initial conditions of Mode 2, i.e. $i_{p}^{k}\left(t_{1}^{k}\right)=I_{\lim }(k)$ and $v_{p}^{k}\left(t_{1}^{k}\right)=a V_{\text {in }}$, the primary side voltage $v_{p}^{k}$, magnetizing inductance current $i_{L_{m}}^{k}$ and primary side current $i_{p}^{k}$ are

$$
\left\{\begin{array}{l}
v_{p}^{k}(t)=a V_{\text {in }} \cos \left[\omega_{o 1}\left(t-t_{1}^{k}\right)\right]-a Z_{r 1} I_{\lim }(k) \sin \left[\omega_{o 1}\left(t-t_{1}^{k}\right)\right] \\
i_{p}^{k}(t)=i_{L_{m}}^{k}(t)=I_{\lim }(k) \cos \left[\omega_{o 1}\left(t-t_{1}^{k}\right)\right]+\frac{V_{\text {in }}}{Z_{r 1}} \sin \left[\omega_{o 1}\left(t-t_{1}^{k}\right)\right]
\end{array}\right.
$$

where

$$
\left\{\begin{array}{l}
\omega_{o 1}=\frac{1}{\sqrt{\left(L_{m}+L_{\mathrm{lk}}\right) C_{r}}} \\
Z_{r 1}=\sqrt{\frac{L_{m}+L_{\mathrm{lk}}}{C_{r}}}
\end{array} .\right.
$$

This mode ends when $v_{p}^{k}(t)=-V_{o}(k-1) / n$. By solving the equation, the time duration $T_{2}^{k}$ of Mode 2 is

$$
T_{2}^{k}=\frac{1}{\omega_{o 1}}\left[\pi-\cos ^{-1}\left(\frac{V_{o}(k-1)}{n a \sqrt{V_{\text {in }}^{2}+\left(Z_{r 1} I_{\text {lim }}(k)\right)^{2}}}\right)-\tan ^{-1}\left(\frac{Z_{r 1} I_{\lim }(k)}{V_{\text {in }}}\right)\right] .
$$

3) Mode $3\left[t_{2}^{k}-t_{3}^{k}\right]$ : When the amplitude of $v_{p}^{k}$ increases to $V_{o}(k-1) / n$, diode $D_{s}$ is conducting with zero-voltage. Hence, the turn-on overshoot voltage is eliminated. The energy is transferred from the primary side to capacitor $C_{o}$. The corresponding equivalent circuit is shown in Fig. 5.3(c).

Given that $C_{r}<<n^{2} C_{o}$, the resonant frequency of primary side current $t_{p}^{k}$ is much higher than that of magnetizing inductance current $i_{L_{m}}^{k}$. In Fig. 5.2, only three oscillation cycles of $i_{p}^{k}$ are demonstrated. The resonance between $L_{1 \mathrm{k}}$ and $C_{r}$ can be described by a second-order differential equation approximately. Referring to the analysis of Mode 2, 
the current $i_{p}^{k}$ and voltage stress $v_{\mathrm{DS}}^{k}$ on MOSFET in the first oscillation during the time interval $t_{2}^{k}-t_{23 \_1}^{k}$ are:

$$
\begin{gathered}
i_{p}^{k}=i_{L_{m}}^{k}(t)=I_{t 2}(k) \cos \left[\omega_{o 2}\left(t-t_{2}^{k}\right)\right]+\frac{1}{Z_{r 2}}\left[V_{\mathrm{in}}+\frac{V_{o}(k-1)}{n}\right] \sin \left[\omega_{o 2}\left(t-t_{2}^{k}\right)\right], \\
v_{\mathrm{DS}}^{k}(t)=V_{\mathrm{in}}+\frac{v_{o}^{k}(t)}{n}+\frac{L_{\mathrm{lk}}}{L_{m}} \cos \left[\omega_{o 2}\left(t-t_{2}^{k}\right)\right]+Z_{r 2} I_{t 2}(k) \sin \left[\omega_{o 2}\left(t-t_{2}^{k}\right)\right]
\end{gathered}
$$

where

$$
\left\{\begin{array}{l}
\omega_{o 2}=\frac{1}{\sqrt{L_{\mathrm{lk}} C_{r}}} \\
Z_{r 2}=\sqrt{\frac{L_{\mathrm{lk}}}{C_{r}}}
\end{array} .\right.
$$

$v_{o}^{k}(t)$ is the output voltage, and $I_{t 2}(\mathrm{k})$ is the primary side current at time $t_{2}^{k}$. When $t_{p}^{k}$ resonates equal to $i_{L m}^{k}$ in the time interval $t_{23_{-} 1}^{k}-t_{23_{-} 2}^{k}$, the energy stored in $L_{\mathrm{lk}}$ can be recycled to charge load capacitor $C_{o}$. Hence, the loss by transformer leakage inductance can be reduced. The current $i_{p}^{k}$ starts the next oscillation cycle from time $t_{23}^{k} 2$. However, the corresponding amplitude decreases because of reduced energy in $L_{\mathrm{lk}}$.

The resonant current between $L_{m}$ and $n^{2} C_{o}$ contributes most of the capacitor charging if $L_{\mathrm{lk}}$ is much smaller than $L_{m}$. Hence, the current $i_{L_{m}}^{k}$ in Mode 3 is approximated as:

$$
i_{L_{m}}^{k}(t) \approx I_{t 2}(k) \cos \left[\omega_{o 3}\left(t-t_{2}^{k}\right)\right]-\frac{V_{o}(k-1)}{n Z_{r 3}} \sin \left[\omega_{o 3}\left(t-t_{2}^{k}\right)\right]
$$

where

$$
\left\{\begin{array}{l}
\omega_{o 3}=\frac{1}{n \sqrt{L_{m} C_{o}}} \\
Z_{r 3}=\frac{1}{n} \sqrt{\frac{L_{m}}{C_{o}}}
\end{array} .\right.
$$


At time $t_{3}^{k}$, the capacitor charging stops. The currents $i_{p}^{k}$ and $i_{L_{m}}^{k}$ are equal to $I_{t 3}(k)$ that is approximately zero. This will be concluded from the operation analysis of Mode 4. Hence, the time duration $T_{3}^{k}$ of Mode 3 is

$$
T_{3}^{k}=\frac{1}{\omega_{o 3}}\left[\frac{\pi}{2}-\tan ^{-1}\left(\frac{V_{o}(k-1)}{n Z_{r 3} I_{t 2}(k)}\right)\right] .
$$

Given that there is no energy loss, the change of output voltage after the $k^{\text {th }}$ charging cycle is determined by

$$
C_{r}\left[V_{\mathrm{in}}^{2}-\left(\frac{V_{o}(k)}{n a}\right)^{2}\right]+\left(L_{m}+L_{\mathrm{lk}}\right)\left[I_{\mathrm{lim}}(k)\right]^{2}=C_{o}\left[V_{o}^{2}(k)-V_{o}^{2}(k-1)\right] .
$$

4) Mode $4\left[t_{3}^{k}-t_{4}^{k}\right]$ : At time $t_{3}^{k}$, diode $D_{s}$ is turned off with zero-current. Hence, the recovery loss is eliminated. Throughout this mode, the diode is blocked. The corresponding equivalent circuit is shown in Fig. 5.3(b). Similar to the analysis of Mode 2, the primary side voltage $v_{p}^{k}$ in Mode 4 is

$$
v_{p}^{k}(t)=-\frac{V_{o}(k)}{n} \cos \left(\omega_{o 1}\left(t-t_{3}^{k}\right)\right)-a Z_{r 1} I_{t 3}(k) \sin \left(\omega_{o 1}\left(t-t_{3}^{k}\right)\right) .
$$

In Mode 4, the amplitude of $v_{p}^{k}$ is smaller than $V_{o}(k) / n$. From (5.14), it can be concluded that the current $I_{t 3}(k)$ at time $t_{3}^{k}$ is approximately zero. Hence, the primary side voltage $v_{p}^{k}$ is simplified as

$$
v_{p}^{k}(t)=-\frac{V_{o}(k)}{n} \cos \left(\omega_{o 1}\left(t-t_{3}^{k}\right)\right)
$$

The voltage stress $v_{\mathrm{DS}}^{k}$ on MOSFET in Mode 4 is

$$
v_{\mathrm{DS}}^{k}(t)=V_{\mathrm{in}}+\frac{V_{o}(k)}{n a} \cos \left(\omega_{o 1}\left(t-t_{3}^{k}\right)\right)
$$


The currents $i_{p}^{k}$ and $i_{L_{m}}^{k}$ are

$$
i_{p}^{k}(t)=i_{L_{m}}^{k}(t)=-\frac{V_{o}(k)}{n a Z_{r 1}} \sin \left(\omega_{o 1}\left(t-t_{3}^{k}\right)\right)
$$

For VVS, the MOSFET is turned on at time $t_{4}^{k}$ for the next charging cycle, and valley-voltage-switching (VVS) can minimize the switching-on loss and current spikes. From (5.16), the valley-voltage is $V_{\text {in }}-V_{o}(k) /(n a)$, and the time duration $T_{4-\mathrm{Vv}}^{k}$ of Mode 4 is

$$
T_{4-\mathrm{VVS}}^{k}=\frac{\pi}{\omega_{o 1}}
$$

From (5.17) and (5.18), the ending current $I_{\text {end-vvs }}(k)$ of $k^{\text {th }}$ charging cycle and initial current $I_{\text {ini }}(k+1)$ of next charging cycle are zero, i.e.

$$
I_{\text {end-VVS }}(k)=I_{\text {ini }}(k+1)=0 \text {. }
$$

For ZVS, the MOSFET is turned on with zero-voltage at time $t_{4}^{k}$ when $V_{o}(k) \geq n a V_{\text {in }}$ from (5.16). By solving $v_{\mathrm{DS}}^{k}(t)=0$, the time duration $T_{4-Z \mathrm{VS}}^{k}$ of Mode 4 is

$$
T_{4-\mathrm{ZVS}}^{k}=\frac{1}{\omega_{o 1}}\left[\pi-\cos ^{-1}\left(\frac{n a V_{\text {in }}}{V_{o}(k)}\right)\right] .
$$

From (5.17) and (5.20), the ending current $I_{\text {end-ZVS }}(k)$ of $k^{\text {th }}$ charging cycle and the initial current $I_{\text {ini }}(k+1)$ of next charging cycle are

$$
I_{\text {end-ZVS }}(k)=I_{\text {ini }}(k+1)=-\frac{1}{n \omega_{o 1} L_{m}} \sqrt{V_{o}^{2}(k)-\left(n a V_{\text {in }}\right)^{2}} .
$$

\subsubsection{Operation Discussion}

This section discusses the quasi-resonant operation to improve capacitor charging efficiency using a quasi-resonant flyback converter. 


\section{A. Maximum Output Voltage $V_{o, \max }$}

From (5.4), a charging current limit $I_{\lim }$ can generate a maximum voltage $V_{o, \max }$ :

$$
V_{o, \max }=n a \sqrt{V_{\mathrm{in}}^{2}+\left(Z_{r 1} I_{\mathrm{lim}}\right)^{2}}
$$

The output voltage of quasi-resonant converter can be adjusted by charging current.

\section{B. Charging Current Limit $I_{\lim }(k)$}

To improve the capacitor charging efficiency, the energy in transformer leakage inductance is prevented being dissipated by the freewheeling diode after the MOSFET is turned off. Hence, the voltage $v_{\mathrm{DS}}^{k}$ across MOSFET should keep positive in Mode 3. As aforementioned discussion, the oscillation amplitude of $v_{\mathrm{DS}}^{k}$ between $t_{23_{-} 1}^{k}-t_{23_{-} 2}^{k}$ is larger than others, and it is the most probable that energy loss occurs. Consequently, it is derived that

$$
V_{\text {in }}+\frac{V_{o}(k-1)}{n}-\sqrt{\left[\frac{L_{\mathrm{lk}}}{L_{m}} \frac{V_{o}(k-1)}{n}\right]^{2}+\left[Z_{r 2} I_{t 2}(k)\right]^{2}} \geq 0
$$

from (5.8). By (5.4) and (5.6), the current $I_{t 2}(k)$ is known as

$$
I_{t 2}(k)=\frac{1}{Z_{r 1}} \sqrt{V_{\mathrm{in}}^{2}+\left[Z_{r 1} I_{\lim }(k)\right]^{2}-\left[\frac{V_{o}(k-1)}{n a}\right]^{2}} .
$$

From (5.23) and (5.24), the charging current limit $I_{\lim }(k)$ in the $k^{\text {th }}$ charging cycle is constrained by

$$
I_{\lim }(k) \leq \frac{1}{Z_{r 2}} \sqrt{a V_{\mathrm{in}}^{2}+\frac{1}{a}\left[\frac{V_{o}(k-1)}{n}\right]^{2}+\frac{2}{n} V_{\mathrm{in}} V_{o}(k-1)} .
$$

With the increase in output voltage, the charging current limit can go higher.

\section{Resonant Capacitance $C_{r}$}

Unlike the discussions on quasi-resonant flyback converters in [77-79], this thesis presents the effect of resonant capacitance. To minimize the switching-off loss, the 
MOSFET is fully turned off without voltage stress reflected from the load capacitor, and the voltage stress is equal to the input voltage $V_{\text {in. }}$ Thereby, the turn-off time $T_{\text {off }}$ of MOSFET should be shorter than the time duration $T_{2}^{k}$ of Mode 2 when secondary side diode $D_{s}$ is blocked. By (5.6), resonant capacitance $C_{r}$ can be known by solving

$$
T_{2}^{k}>T_{\text {off }}
$$

The total switching-off loss is

$$
W_{\text {sw-off }}=\frac{1}{2} V_{\text {in }} T_{\text {off }} \sum_{k} I_{\text {lim }}(k) .
$$

\section{ZVS and Hybrid Switching}

If the output voltage $V_{o}(1)$ can be higher than $n a V_{\text {in }}$ after the first charging cycle, ZVS can be obtained throughout the capacitor charging process. From (5.13), the charging current limit $I_{\lim }(1)$ of first charging cycle should be higher than $I_{L}$ :

$$
I_{L}=n a \sqrt{\frac{C_{o}}{L_{m}+L_{1 \mathrm{k}}}} V_{\mathrm{in}} .
$$

From (5.25), the charging current limit $I_{\lim }(1)$ should be lower than $I_{H}$ :

$$
I_{H}=\frac{V_{\mathrm{in}}}{Z_{r 2}} \sqrt{\frac{L_{m}}{L_{m}+L_{\mathrm{lk}}}} .
$$

Hence, ZVS throughout the charging process requires a resonant capacitance larger than $C_{r, \mathrm{ZVS}-\mathrm{min}}$ which is derived from (5.28) and (5.29) as

$$
C_{r, \text { ZVS-min }}=\left(\frac{n L_{m}}{L_{m}+L_{\mathrm{lk}}}\right)^{2} \frac{L_{\mathrm{lk}}}{L_{m}} C_{o} .
$$

When the charging current limit is lower than $I_{L}$, or resonant capacitance $C_{r}$ is smaller

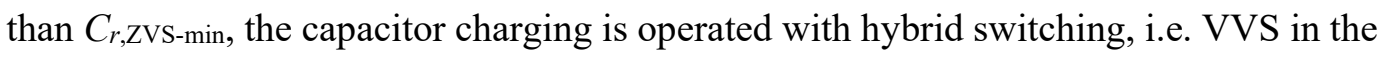
first few charging cycles and ZVS afterwards. 
The switching-on loss is eliminated by ZVS operation. For VVS, there exists a switching-on loss $W_{\text {sw-on: }}$

$$
W_{\mathrm{sw}-\mathrm{on}}=\frac{1}{2} C_{r} \sum_{k=1}^{N_{\mathrm{VVS}}}\left[V_{\mathrm{in}}-\frac{V_{o}(k)}{n a}\right]^{2}
$$

where $N_{\mathrm{vvs}}$ is the total number of charging cycles using VVS.

\subsection{Prototype Development}

\subsubsection{Key Components}

From (5.22), a smaller leakage inductance allows a larger voltage gain by a given charging current limit. Hence, the target of designing a transformer in this chapter is to make the leakage inductance as small as possible. The approach is to coil the primary winding by connecting top and bottom layers in parallel. Between them is coiled the secondary winding $[116,121]$. The parameters of transformer (magnetizing inductance $L_{m}$, leakage inductance $L_{\mathrm{lk}}$ and winding capacitance $C_{w}$ ) can be extracted by an LCR meter using the method discussed in Section 4.2.1. Table 5.1 shows parameters of a custom made transformer with a single secondary winding.

The selected diode RS3M-13-F has a peak repetitive reverse voltage of $1 \mathrm{kV}$. To assure the margin of reverse voltage, two diodes are connected in series. A MOSFET STB28NM50N with a drain-source breakdown voltage of $500 \mathrm{~V}$ is selected as the power switch. 
Table 5.1 Components used in the quasi-resonant flyback converter

\begin{tabular}{l|l|c}
\hline \hline \multirow{5}{*}{ Transformer } & Ferrite core & B66423GX187 \\
\cline { 2 - 3 } & Winding capacitance $C_{w}$ & $0.142 \mathrm{nF}$ \\
\cline { 2 - 3 } & Magnetizing inductance $L_{m}$ & $56.1 \mu \mathrm{H}$ \\
\cline { 2 - 3 } & Turns of primary winding & \multicolumn{1}{c}{40} \\
\cline { 2 - 3 } & Turns of secondary winding & $0.3 \mu \mathrm{H}$ \\
\cline { 2 - 3 } & Leakage inductance $L_{\mathrm{lk}}$ & $64.67 \mathrm{~m} \Omega$ \\
\cline { 2 - 3 } & DC resistance $R_{\mathrm{dc}}$ & $8 \mathrm{~A}$ \\
\cline { 2 - 3 } & Saturation current $I_{\mathrm{sat}}$ & $\mathrm{RS} 3 \mathrm{M}-13-\mathrm{F}$ \\
\hline \multirow{5}{*}{ Diode $D_{s}$} & Part number & $1.3 \mathrm{~V}$ \\
\cline { 2 - 3 } & Forward voltage $V_{\mathrm{F}}$ & $1 \mathrm{kV}$ \\
\cline { 2 - 3 } & Peak repetitive reverse voltage $V_{\mathrm{PRM}}$ & $43.3 \mathrm{~m} \Omega$ \\
\cline { 2 - 3 } & On-resistance $R_{\mathrm{D}}$ & $\mathrm{STB} 28 \mathrm{NM} 50 \mathrm{~N}$ \\
\hline & Part number & $135 \mathrm{~m} \Omega$ \\
\cline { 2 - 3 } & Drain-source on-resistance $R_{\mathrm{on}}$ & $1.5 \mathrm{~V}$ \\
\cline { 2 - 3 } & Freewheeling diode threshold voltage $V_{\mathrm{SD}}$ & $500 \mathrm{~V}$ \\
\cline { 2 - 3 } & Drain-source breakdown voltage $V_{\mathrm{BD}}$ & $71.4 \mathrm{~m} \Omega$ \\
\cline { 2 - 3 } & Freewheeling diode on-resistance $R_{\mathrm{SD}}$ & $418 \mathrm{pF}$ \\
\cline { 2 - 3 } & Output capacitance $C_{\text {oss }}$ & $32.6 \mathrm{~ns}$ \\
\cline { 2 - 3 } & Turn-on time $T_{\text {on }}$ & $114 \mathrm{~ns}$ \\
\cline { 2 - 3 } & Turn-off time $T_{\text {off }}$ & \\
\hline \hline
\end{tabular}

\subsubsection{Control Circuit}

Fig. 5.4 shows the block diagram of control circuit. The input current $i_{\text {in }}$ is monitored by a $10 \mathrm{~m} \Omega$ sensing resistor $R_{\text {sense }}$ shown in Fig. 5.1 . When the input current $i$ in reaches the charging current limit $I_{\mathrm{lim}}$, the MOSFET is turned off by a comparator. The zerovoltage transition and output voltage are detected by a resistor voltage divider in the primary side shown in Fig. 5.1.

As discussed in Section 5.2, the hybrid switching control is employed if the resonant

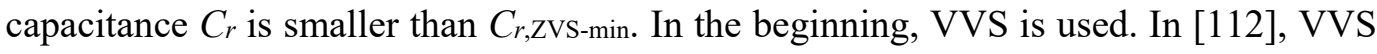
is executed after a delay time when the voltage across the MOSFET drops below the input voltage $V$ in. The delay time is determined by calculation and test. However, the circuit does not perform VVS exactly because the magnetizing inductance changes nonlinearly with the current flowing into the transformer. This is caused by the nonlinear relationship between the flux density $(\boldsymbol{B})$ and magnetic field strength $(\boldsymbol{H})$, i.e. the $\boldsymbol{B}-\boldsymbol{H}$ curve. Hence, the time when the valley-voltage occurs varies with different operational 
conditions. In [122], VVS is conducted when the voltage stress on the MOSFET is smaller than a fixed reference voltage. For the capacitor charging application, the valleyvoltage varies in the process. Hence, this approach cannot perform VVS exactly either.

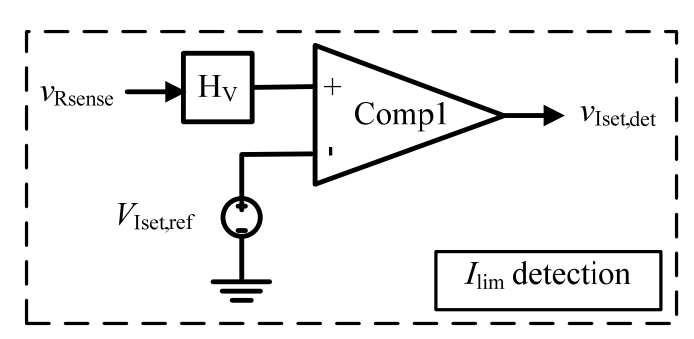

(a)

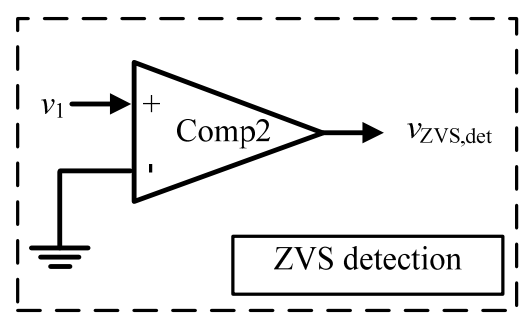

(b)

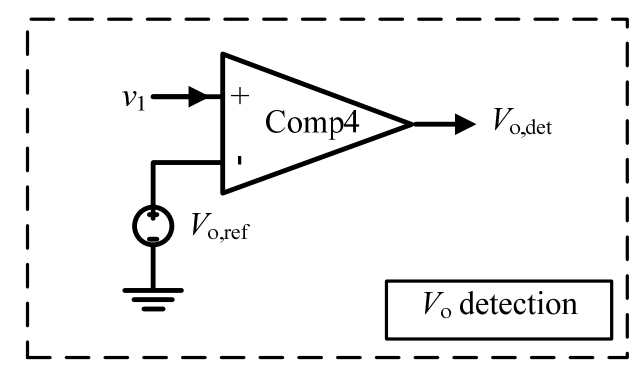

(c)

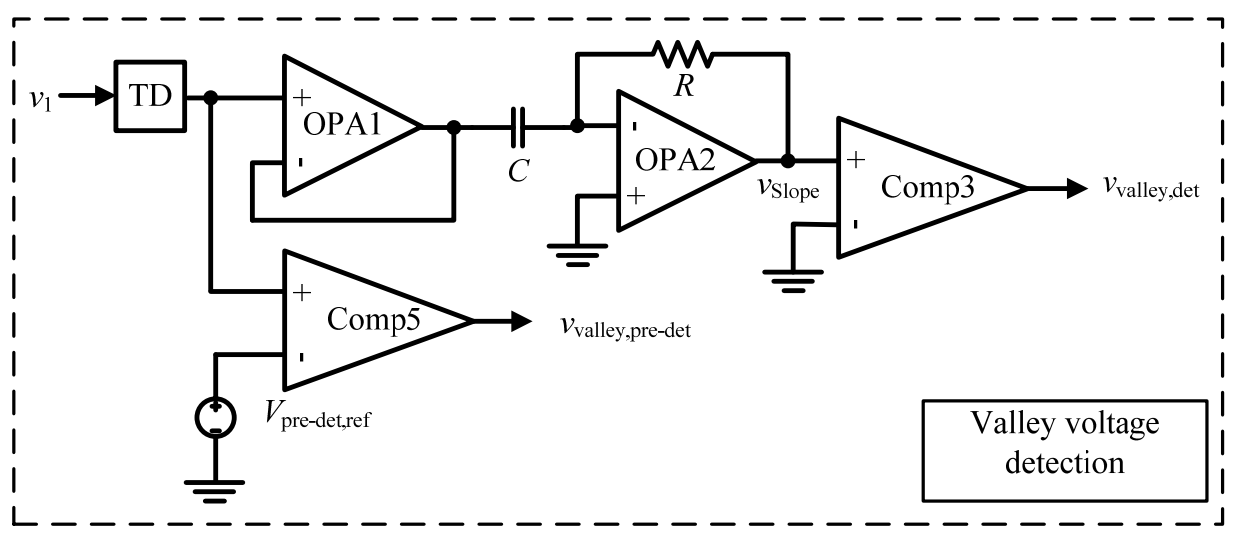

(d)

Fig. 5.4 Control circuit for (a) $I_{\text {lim }}$ detection, (b) zero-voltage transition detection, (c) output voltage $V_{o}$ monitor, and (d) valley-voltage transition detection.

In the prototype circuit, the valley-voltage detection circuit is shown in Fig. 5.4(d), and the logic control signals are shown in Fig. 5.5. The voltage $v_{\text {DS }}$ across the MOSFET is monitored by $v_{1}$ which is divided from the resistor divider formed by $R_{1}$ and $R_{2}$ shown 
in Fig. 5.1. Since the valley-voltage is smaller than $V_{\text {in, }} V_{\text {pre-det,ref }}$ is set to $\frac{R_{2}}{R_{1}+R_{2}} V_{\text {in }}$. When the divided voltage $v_{1}$ decreases to smaller than $V_{\text {pre-det,ref }}$ during $t_{3}^{k}-t_{4}^{k}$ (Mode 4), the circuit starts to detect the valley-voltage. However, the oscillation during $t_{2}^{k}-t_{3}^{k}$ (Mode 3) may cause fault detection as demonstrated in Fig. 5.5. In practice, the voltage oscillation of $v_{\mathrm{DS}}$ during $t_{2}^{k}-t_{3}^{k}$ will be damped by the transformer DC resistor $R_{\mathrm{DC}}$. The damping attenuation factor $\zeta$ is

$$
\zeta=\frac{R_{\mathrm{DC}}}{2 L_{\mathrm{lk}}} .
$$

To avoid fault detection, the valley-voltage detection starts after a time delay $t_{\mathrm{TD}}$ that the oscillation is damped. By the parameters listed in Table 5.1, the delay time can be $28 \mu$ s to make $e^{-\zeta} \approx 0.05(\zeta \approx 3)$. With a time delay $t_{\mathrm{TD}}$, a voltage lower than $V_{\text {pre-det,ref }}$ is guaranteed to be detected in the time interval $t_{3}^{k}-t_{4}^{k}$, and valid for the valley-voltage detection.

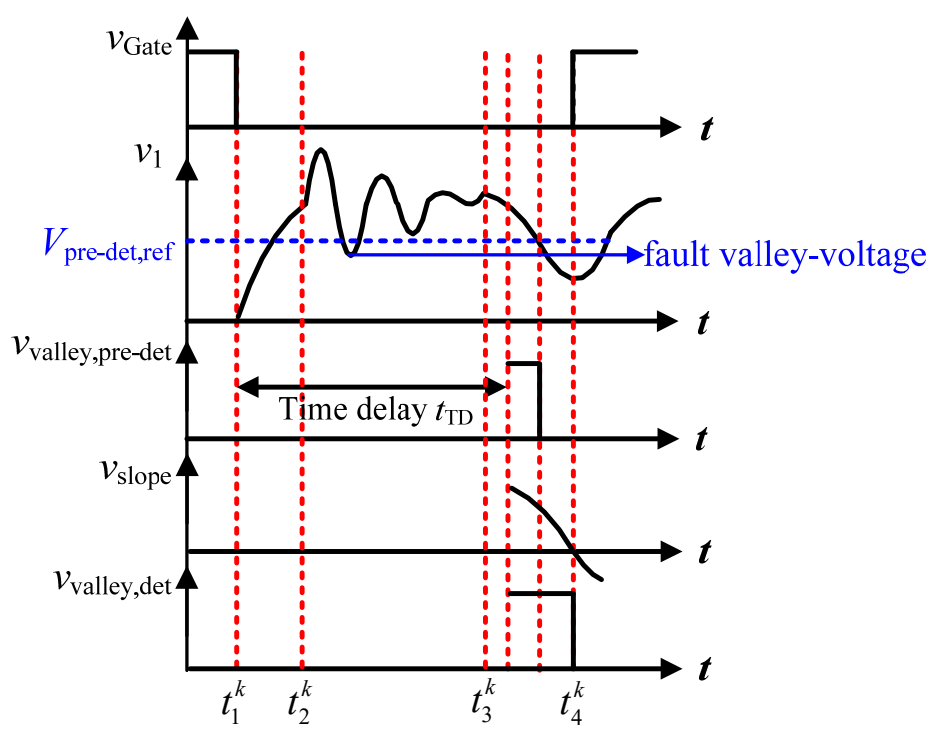

Fig. 5.5 The logic control of valley-voltage-switching (VVS). 
Operational amplifier OPA1 behaves a buffer. Resistor $R$, capacitor $C$ and operational amplifier OPA2 form a differential circuit which generates $v_{\text {slope }}$ determined by

$$
v_{\text {slope }}=-R C \frac{d v_{1}}{d t}
$$

The valley-voltage occurs when the zero crossing of $v_{\text {slope }}$ is detected. By this way, the VVS is performed more exactly compared to the methods discussed in $[112,122]$.

The software flow chart is depicted in Fig. 5.6. The default switching control is VVS in the beginning. After a time delay, the circuit starts to detect the valley-voltage and zero-voltage transitions. If the zero-voltage transition is detected, the switching control is switched to ZVS. In ZVS mode, the time delay is not required. Once the objective output voltage $V_{\text {obj }}$ is obtained, the charging process ends.

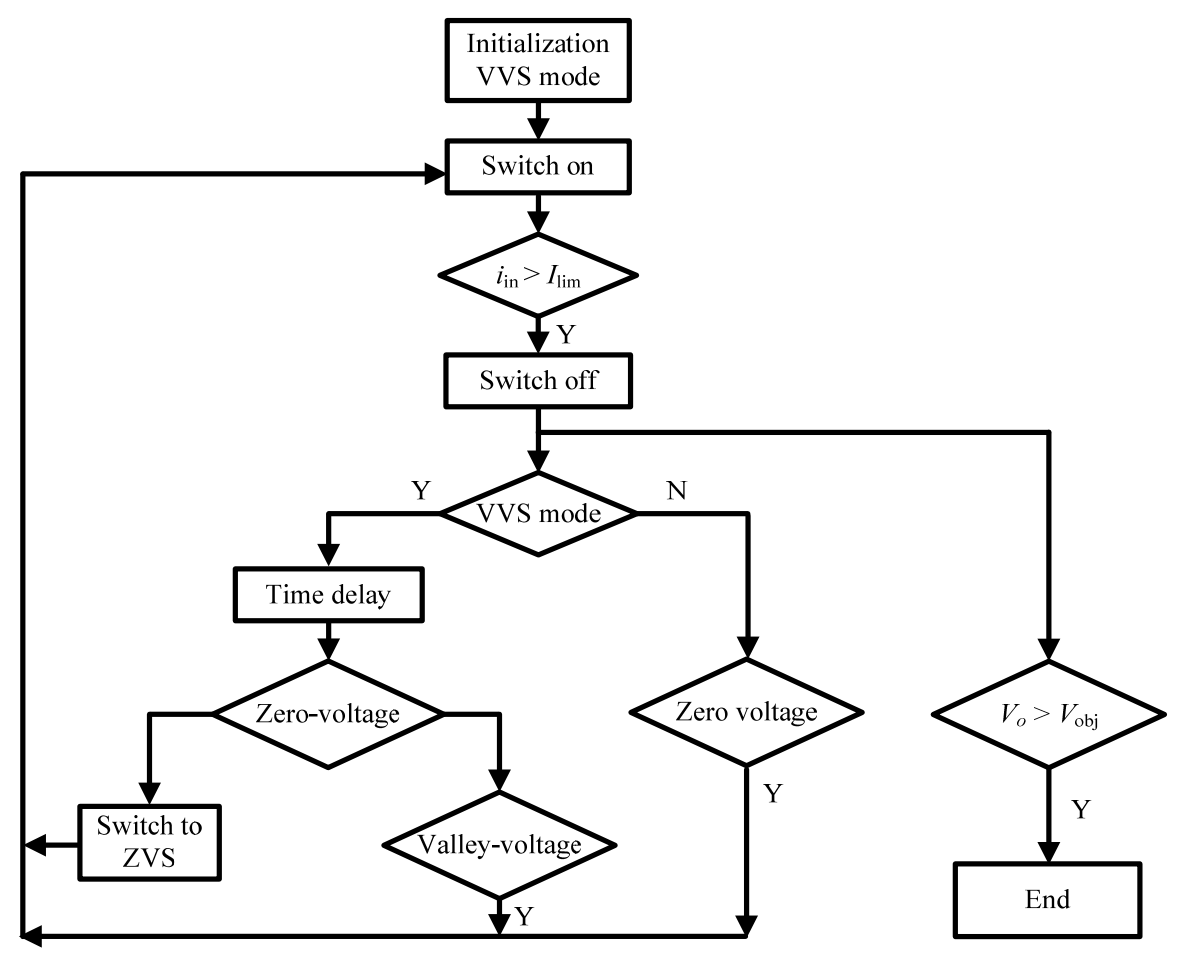

Fig. 5.6 Software flowchart of switching control for capacitor charging. 


\subsection{Simulation and Experimental Results}

A prototype circuit has been developed shown in Fig. 5.7. To validate the proposed concept for capacitor charging, simulation and experiment have been conducted. The results are presented in the following sections.

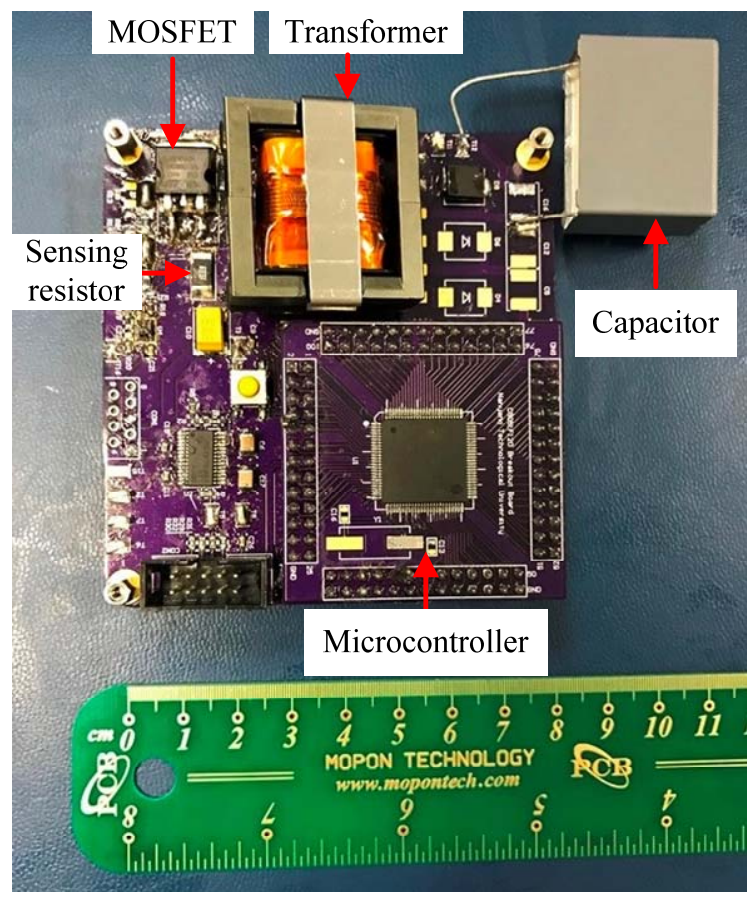

Fig. 5.7 Prototype circuit of quasi-resonant flyback converter.

\subsubsection{ZVS throughout Capacitor Charging Process}

As discussed in Section 5.2.2, resonant capacitance $C_{r}$ is larger than $C_{r \text {,ZVS-min given }}$ in (5.30) if the quasi-resonant flyback converter is operated with ZVS throughout the capacitor charging process. From the parameters listed in Table 5.1, resonant capacitance $C_{r}$ should be larger than $132.3 \mathrm{nF}$ if load capacitance $C_{o}$ is $1 \mu \mathrm{F}$. In the prototype circuit, a capacitor $C_{\mathrm{in}}$ of $200 \mathrm{nF}$ is connected in parallel with the primary side 
of transformer. Hence, the resonant capacitance $C_{r}$ is $200.56 \mathrm{nF}$ lumped from capacitor $C_{\mathrm{in}}$, transformer winding capacitance $C_{w}$ and MOSFET output capacitance $C_{\text {oss. }}$.

Besides, to realize ZVS throughout the charging process, the charging current limit $\operatorname{Iim}(1)$ of the first charging cycle is constrained by $3.31 \mathrm{~A} \leq I_{\lim }(1) \leq 4.08 \mathrm{~A}$ from (5.28) and (5.29). The charging current can be increased but constrained by (5.25). In this experiment, the charging current is fixed to $3.8 \mathrm{~A}$. Using (5.6), the time duration $T_{2}^{k}$ of Mode 2 is $0.29 \mu \mathrm{s}$. This is longer than the turn-off time of MOSFET which is $114 \mathrm{~ns}$ shown in Table 5.1. Hence, this resonant capacitance can reduce switching-off loss as discussed in Section 5.2.2.

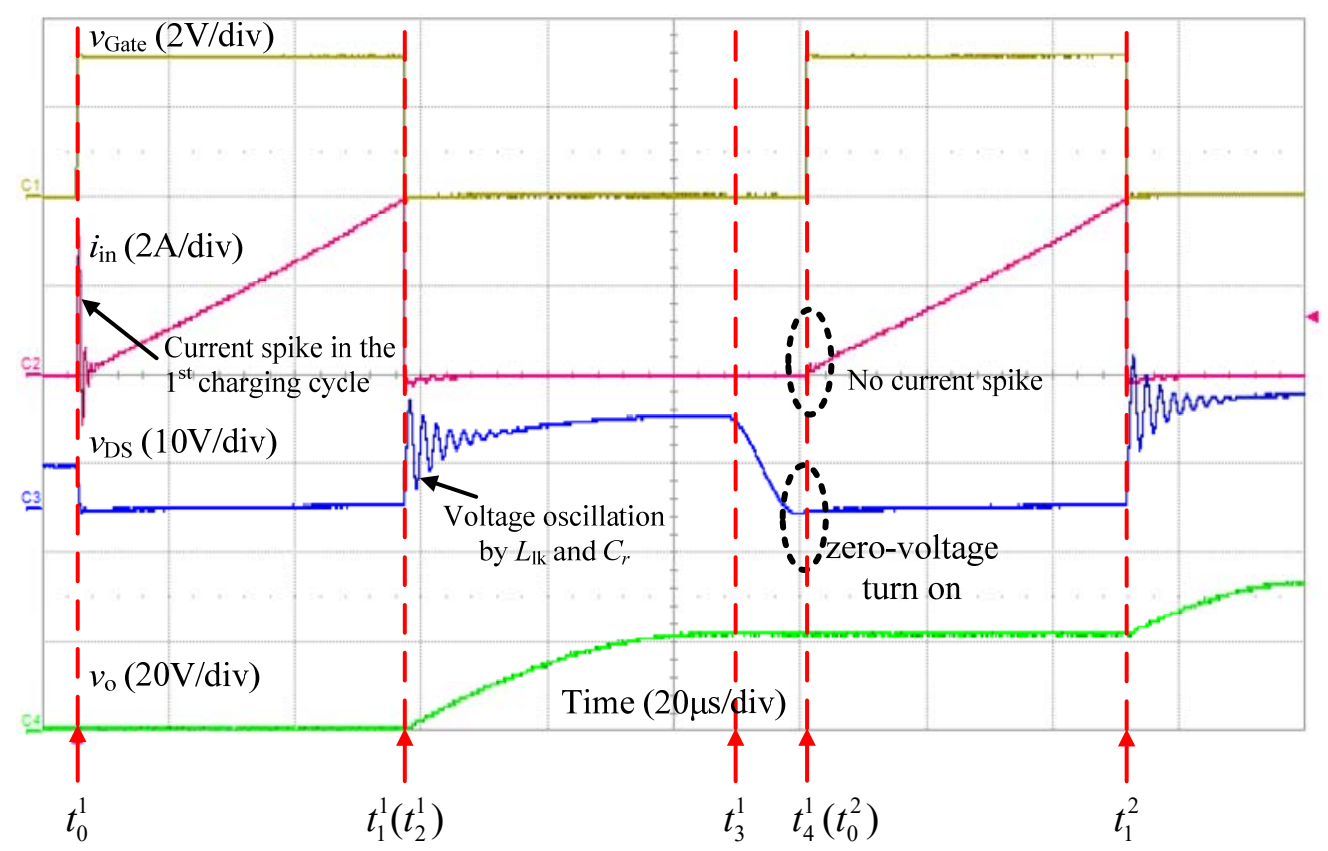

Fig. 5.8 Experimental waveforms of the first two charging cycles when $I_{\text {lim }}=3.8 \mathrm{~A}, C_{r}=200.56 \mathrm{nF}$, and $C_{o}=1 \mu \mathrm{F}$. $v_{\text {Gate: }}$ gating signal, $i_{\text {in: }}$ input current, $v_{\mathrm{DS}}$ : voltage across MOSFET, $v_{\mathrm{o}}$ : output voltage.

Fig. 5.8 shows the experimental waveforms of the first two charging cycles. Initially, the voltage across the resonant capacitor is zero. Hence, there are inrush current spikes when the MOSFET is turned on the first time. When the input current $i_{\text {in }}$ increases 
linearly to $I_{\mathrm{lim}}$, the MOSFET is turned off. As the time duration of Mode 2 in the first charging cycle is too short to distinguish, $t_{1}^{1}$ and $t_{2}^{1}$ are labelled together in Fig. 5.8. In Mode $3\left(t_{2}^{1}-t_{3}^{1}\right)$, there is a voltage oscillation on the MOSFET which is caused by leakage inductance $L_{\mathrm{lk}}$ and lumped resonant capacitance $C_{r}$. As load capacitor $C_{o}$ is charged, the output voltage increases in this mode. It is observed that the voltage stress $v_{\mathrm{DS}}$ on MOSFET keeps positive, and the input current $i_{\text {in }}$ remains zero in Mode 3 . Hence, no energy is dissipated by the freewheeling diode. The experimental results coincide with the theoretical analysis. In Mode $4\left(t_{3}^{1}-t_{4}^{1}\right)$, the voltage stress $v_{\mathrm{DS}}$ decreases until zero where the MOSFET is turned on to obtain ZVS for the next charging cycle. It is observed that the inrush current spikes are eliminated from the second charging cycle and onwards due to ZVS. Hence, the switching-on loss is eliminated, and the reliability of MOSFET can be improved.

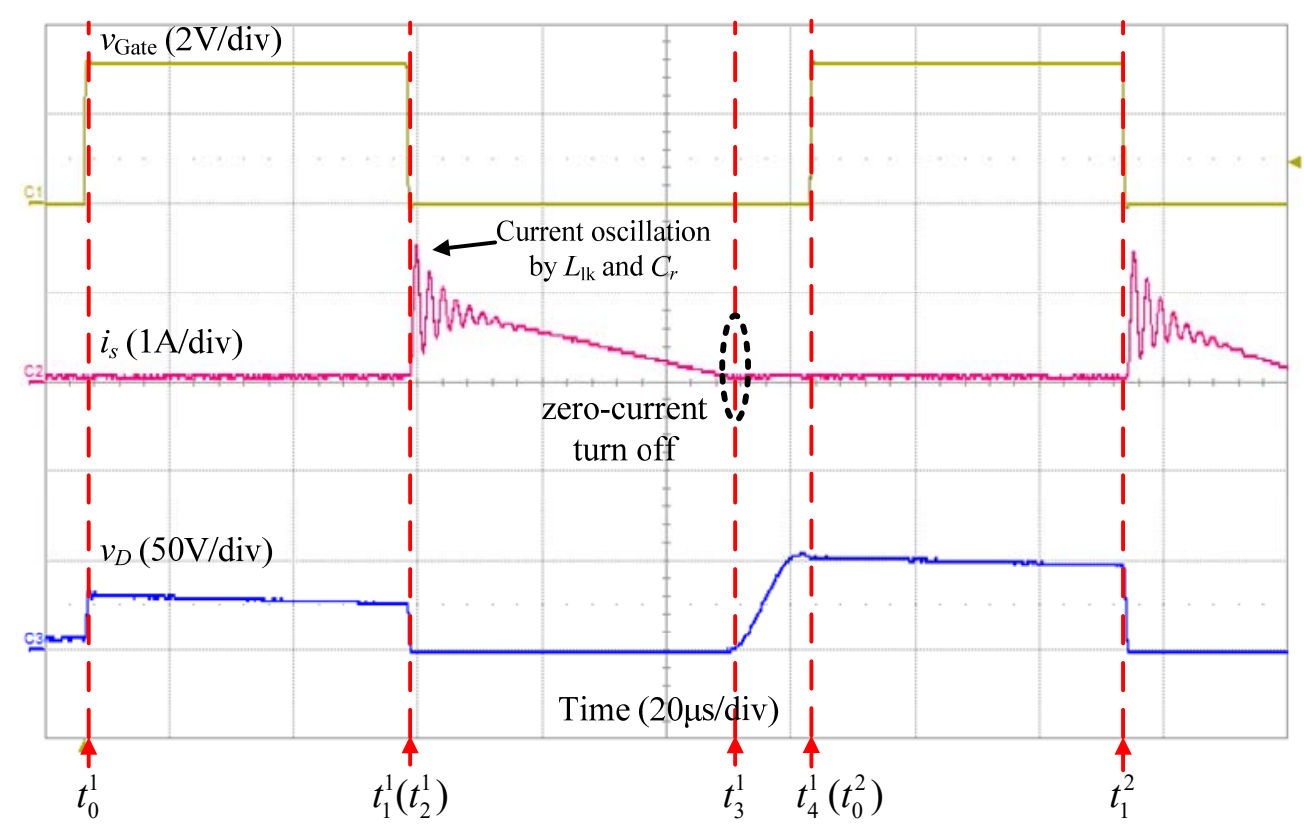

Fig. 5.9 Experimental waveforms of the first two charging cycles when $I_{\mathrm{lim}}=3.8 \mathrm{~A}, C_{r}=200.56 \mathrm{nF}$, and $C_{o}=1 \mu \mathrm{F}$. $v_{\text {Gate: }}$ gating signal, $i_{s}$ : secondary side current, $v_{D}$ : voltage stress on diode $D_{s}$. 
Fig. 5.9 shows the secondary side current $i_{s}$ and reverse voltage $v_{D}$ on diode $D_{s}$. Due to the operation in Mode $2\left(t_{1}^{1}-t_{2}^{1}\right)$, diode $D_{s}$ is not conducted instantly when the MOSFET is turned off. It is turned on with zero-voltage at time $t_{2}^{1}$. Hence, the turn-on overshoot voltage is eliminated as compared to a conventional flyback converter. During the time interval $t_{2}^{1}-t_{3}^{1}$ (Mode 3 ), diode $D_{s}$ is conducted, and load capacitor $C_{o}$ is charged. Due to transformer leakage inductance $L_{\mathrm{lk}}$ and resonant capacitance $C_{r}$, there is a current oscillation. At time $t_{3}^{1}$, diode $D_{s}$ is off with zero-current. Hence, the recovery loss is reduced.

The measured maximum voltage stresses $v_{D}$ on diode $D_{s}$ in the first two charging cycles are $25.4 \mathrm{~V}\left(t_{0}^{1}-t_{1}^{1}\right)$ and $52.1 \mathrm{~V}\left(\left(t_{0}^{2}-t_{1}^{2}\right)\right)$. The corresponding analytical results are 24.87 $\mathrm{V}$ and $53.38 \mathrm{~V}$ by (5.1) and (5.13). The small difference of $3 \%$ also validates the theoretical analysis.

It is noted that diode $D_{s}$ is always conducting in Mode $3\left(t_{2}^{1}-t_{3}^{1}\right)$ in the first two charging cycles, and the current oscillation ends shortly. The experimental waveform of $i_{s}$ in Fig. 5.9 is different from that in Fig. 5.2. This is because all the energy stored in leakage inductance is dissipated by the circuit DC resistor, and not recycled to charge the load capacitor. Consequently, the time interval $t_{2}^{1}-t_{23_{-} 1}^{1}\left(t_{2}^{2}-t_{23_{-} 1}^{2}\right)$ in Fig. 5.2 does not exist in the experiment of first two charging cycles.

To verify the explanation, a simulation study has been performed by PSIM software, and the results are shown in Fig. 5.10. Without considering the circuit DC resistor, the primary current $i_{\mathrm{s}}$ drops to zero when the energy is recycled from leakage inductance $L_{\mathrm{k}}$ to magnetizing inductance $L_{m}$. The corresponding simulation waveforms of the first two charging cycles shown in Fig. 5.10(a) coincide with the theoretical analysis based on 
Fig. 5.2. It can be observed that the oscillation amplitude decreases as the energy in $L_{\mathrm{lk}}$ is recycled. Without being damped, the oscillation lasts until the end of Mode 3. Fig. 5.10(b) shows the simulation waveforms with a circuit DC resistor. It is observed that the oscillation is damped with an attenuation factor given in (5.32), and diode $D_{s}$ is always conducting in Mode 3. This agrees well with the experimental results.

Fig. 5.11 shows the simulation waveforms with a circuit DC resistor when the output voltage is higher than $112 \mathrm{~V}$. With the increase in output voltage, the time duration of Mode 3 becomes shorter from (5.12) and (5.24). When the damping attenuation of primary side current $i_{p}^{k}$ is smaller than the decreasing rate of magnetizing inductance current $i_{L_{m}}^{k}$, the time interval $t_{2}^{k}-t_{2 \beta_{-} 1}^{k}$ will turn up, and the energy in leakage inductance can be recycled. This is helpful to reduce energy loss. Moreover, the energy is recycled earlier if the damping attenuation factor is smaller. 


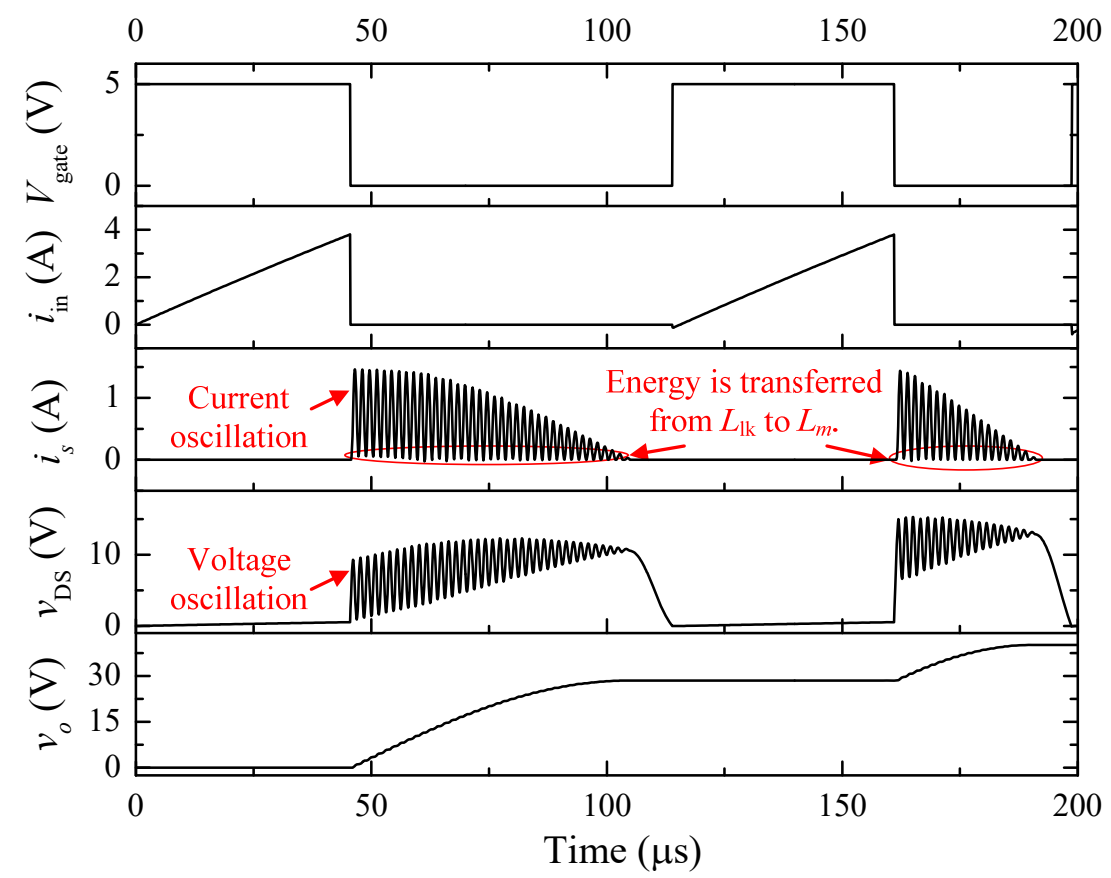

(a)

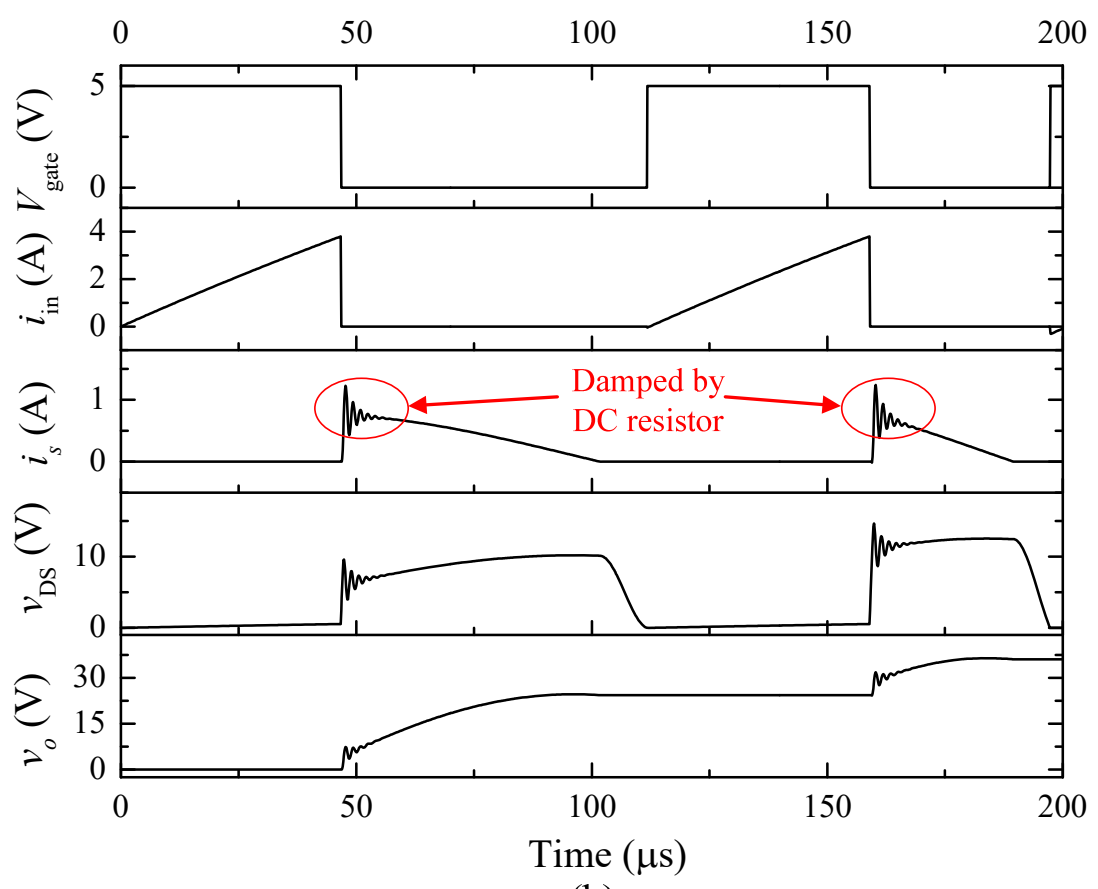

(b)

Fig. 5.10 Simulation waveforms of the first two charging cycles (a) without and (b) with circuit DC resistors when $I_{\lim }=3.8 \mathrm{~A}, C_{r}=200.56 \mathrm{nF}$, and $C_{o}=1 \mu \mathrm{F}$. $v_{\text {Gate: }}$ gating signal, $i_{\text {in }}$ : input current, $v_{\mathrm{DS}}$ : voltage across MOSFET, $v_{0}$ : output voltage. 


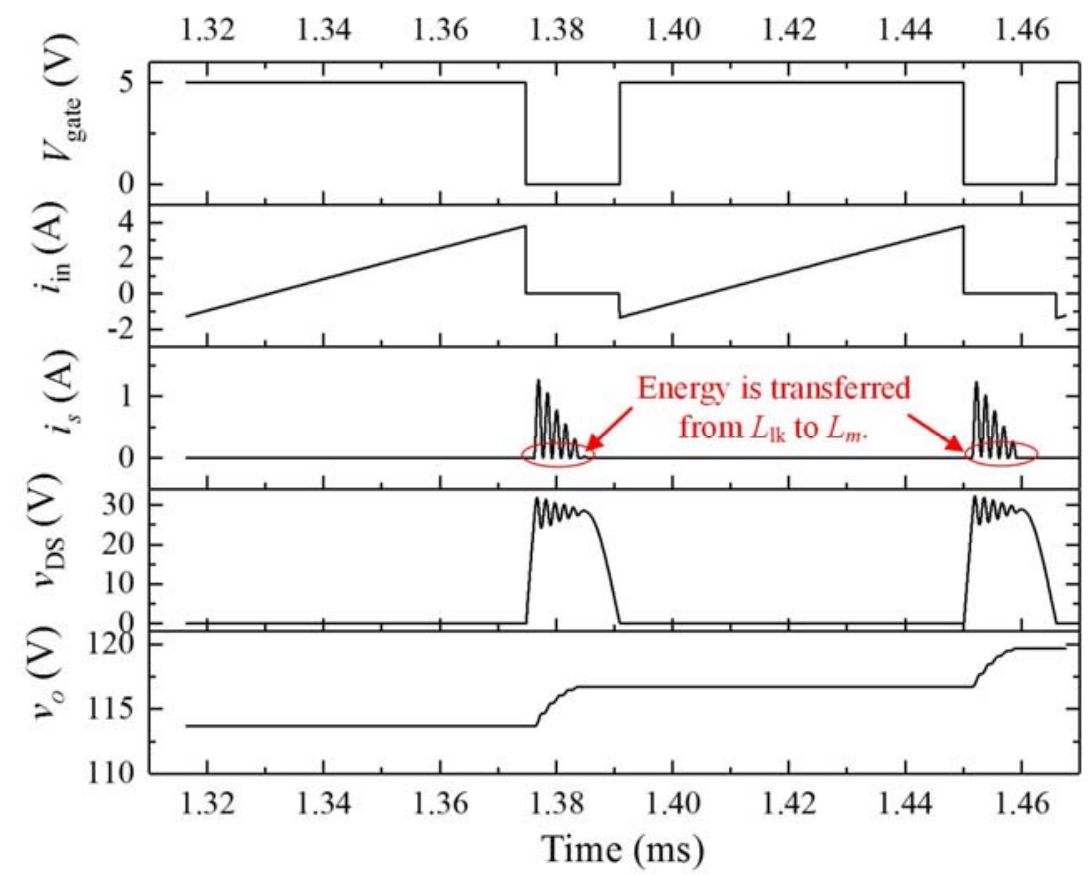

Fig. 5.11 Simulation waveforms when $I_{\lim }=3.8 \mathrm{~A}, C_{r}=200.56 \mathrm{nF}, C_{o}=1 \mu \mathrm{F}$ with circuit DC resistors. $v_{\text {Gate: }}$ gating signal, $i_{\text {in }}$ input current, $v_{\mathrm{DS}}$ : voltage across MOSFET, $v_{0}$ : output voltage.

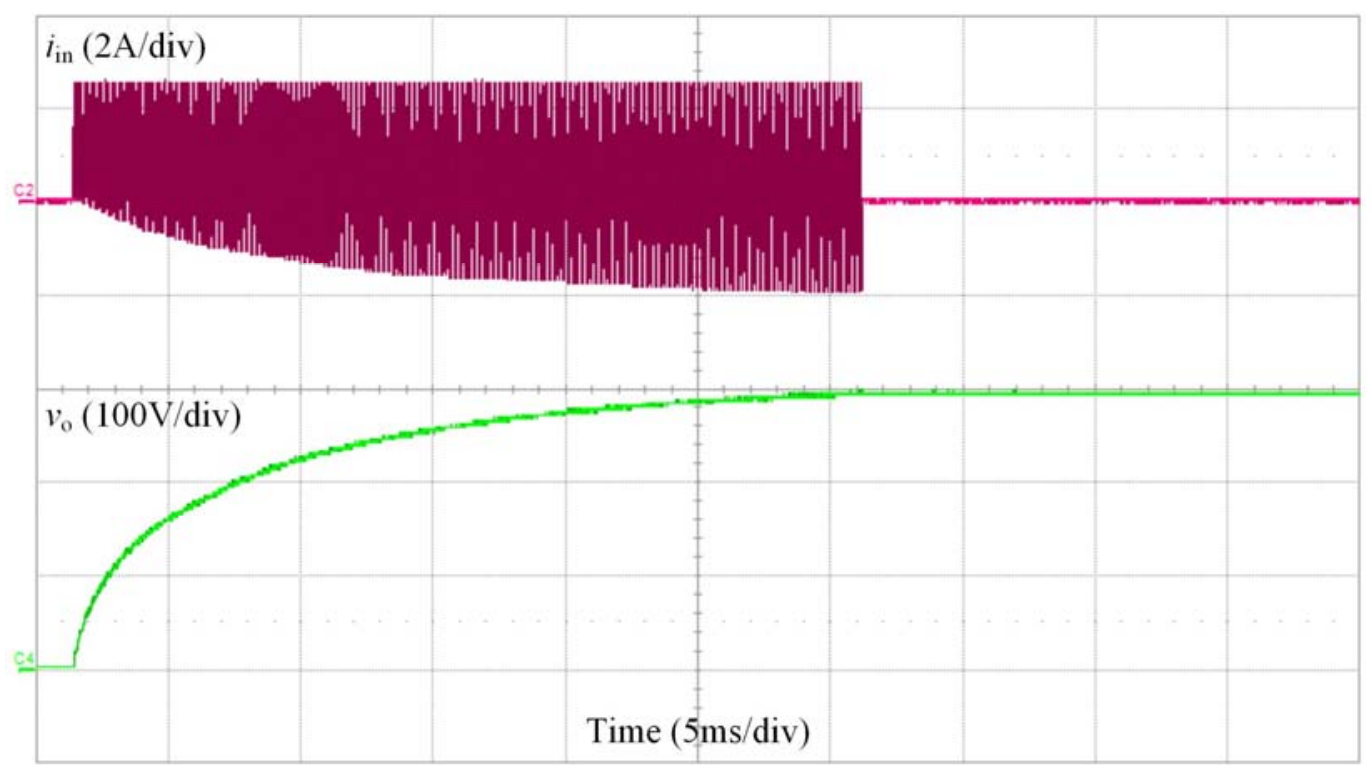

Fig. 5.12 Capacitor charging waveforms when $I_{\lim }=3.8 \mathrm{~A}, C_{r}=200.56 \mathrm{nF}$, and $C_{o}=1 \mu \mathrm{F}$. $i_{\text {in }}$ : input current; $v_{0}$ : output voltage. 
Fig. 5.12 shows waveforms of charging a capacitor of $1 \mu \mathrm{F}$, and it takes about $36 \mathrm{~ms}$ to charge to $300 \mathrm{~V}$. It is observe that the amplitude of initial current $I_{\text {ini }}(k)$ goes larger with the increase in output voltage. This coincides with the theoretical analysis given in (5.21).

After the ZVS operation has been validated by simulation and experiment, its characteristics are next studied.

As discussed in Section 5.2.2, the resonant capacitance $C_{r}$ should be larger than $C_{r, \text { ZVS-min }}$ to achieve ZVS throughout the charging process. Fig. 5.13 shows the results

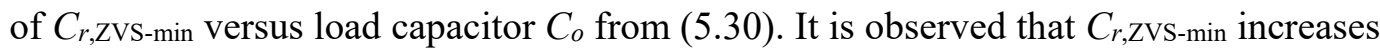
with capacitance $C_{o}$. A bigger resonant capacitance $C_{r}$ leads to the risk of more energy loss if a malfunction of ZVS occurs. This makes the switching control of ZVS throughout the charging process less favorable for a larger capacitance $C_{o}$.

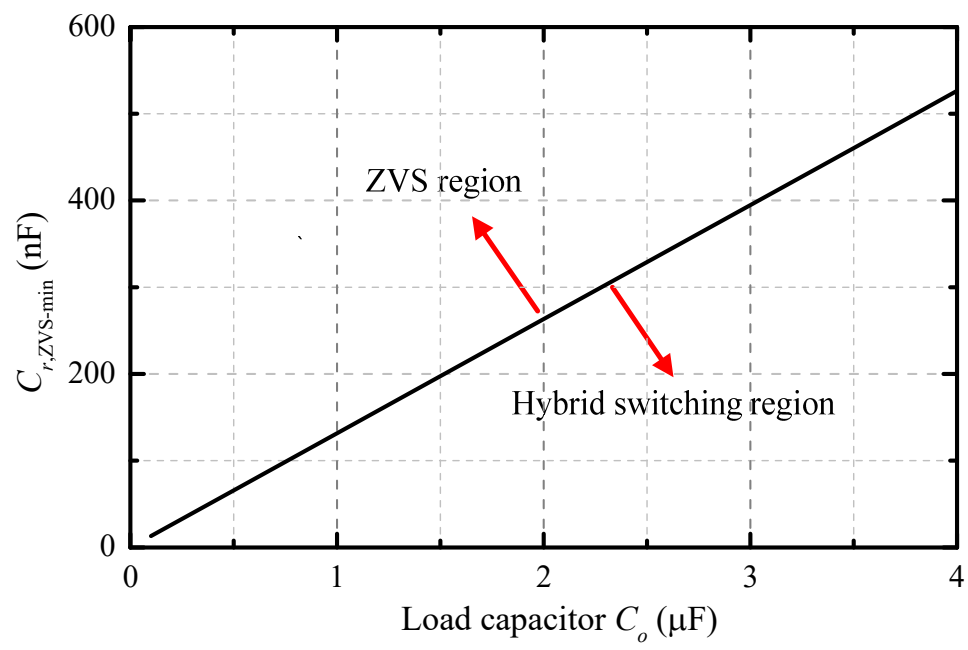

Fig. 5.13 Minimum resonant capacitance $C_{r, \text { ZVS-min }}$ versus the capacitance $C_{o}$ if ZVS is obtained throughout the capacitor charging process. 
Fig. 5.14 shows the range of charging current limit $I_{\text {lim }}(1)$ for the first charging cycle by (5.29) when $C_{o}$ is $1 \mu \mathrm{F}$. It is noted that the lower current limit $I_{L}$ is not affected by $C_{r}$ while the upper current limit $I_{H}$ is increased with $C_{r}$. Limited by the transformer saturation current, there is supposed to be a maximum resonant capacitance. It is observed that a larger $C_{r}$ leads to a wider range of charging current. This makes the switching control implementation easier, because the monitoring accuracy of the charging current has a larger margin.

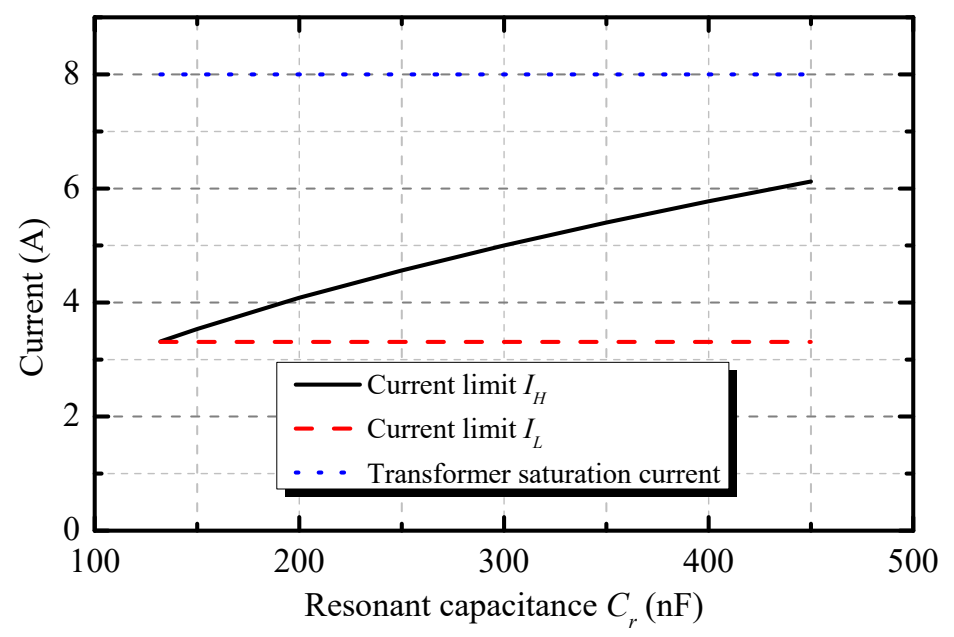

Fig. 5.14 Charging current limit versus resonant capacitance when the load capacitance $C_{o}$ is $1 \mu \mathrm{F}$.

Fig. 5.15 shows the results of maximum output voltage $V_{o, \max }$ versus the charging current limit $I_{\mathrm{lim}}$ by (5.22). Three different resonant capacitances, $150.56 \mathrm{nF}, 200.56 \mathrm{nF}$ and $470.56 \mathrm{nF}$, have been selected for this study. It is observed that a larger resonant capacitance has a smaller output voltage using a given charging current limit. Moreover, the charging current is also limited by other factors, such as saturation current of transformer, current rating of MOSFET, etc. Hence, a smaller resonant capacitance is preferred. 


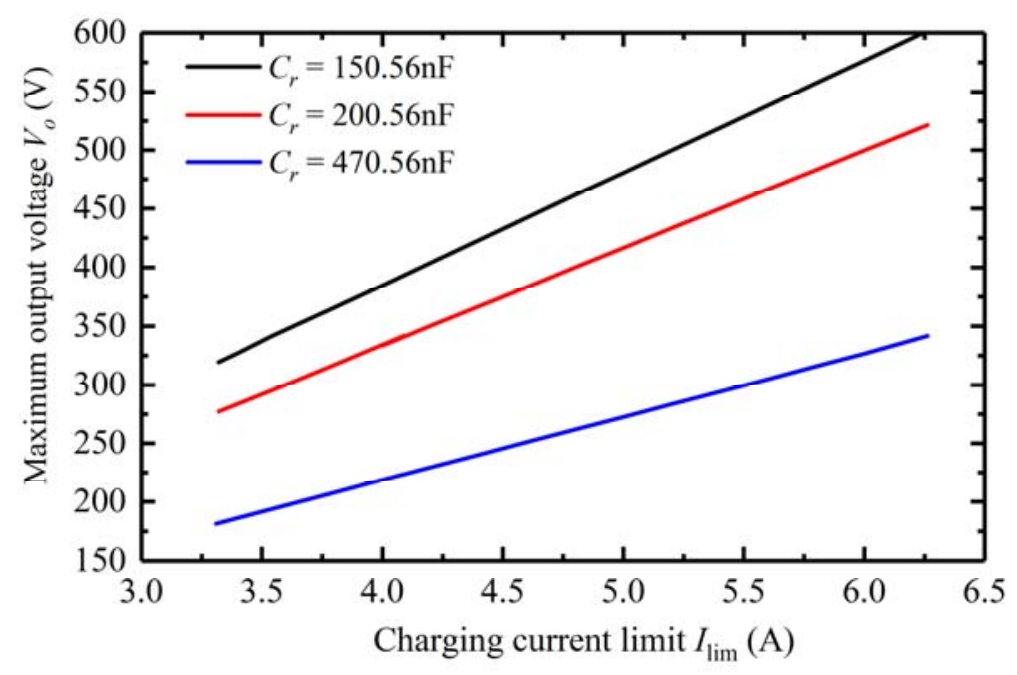

Fig. 5.15 Maximum output voltage $V_{o, \max }$ versus charging current limit $I_{\text {lim. }}$.

The charging time and efficiency are also studied using the scheme that the charging current limit is fixed throughout the charging process. Two resonant capacitances, $200.56 \mathrm{nF}$ and $470.56 \mathrm{nF}$, are selected. The relevant parameters are listed in Table 5.2.

Table 5.2 Parameters for studies on capacitor charging with ZVS

\begin{tabular}{|c|c|c|c|c|c|c|}
\hline$C_{o}(\mu \mathrm{F})$ & $C_{r, \mathrm{ZVS}-\min }(\mathrm{nF})$ & $C_{r}(\mathrm{nF})$ & $I_{L}(\mathrm{~A})$ & $I_{H}(\mathrm{~A})$ & $I_{\lim }(\mathrm{A})$ & $V_{o, \max }(\mathrm{V})$ \\
\hline \multirow{7}{*}{1} & \multirow{7}{*}{131.6} & \multirow{4}{*}{200.56} & \multirow{4}{*}{3.31} & \multirow{4}{*}{4.08} & 3.8 & 317 \\
\hline & & & & & & \\
\hline & & & & & 4.08 & 340 \\
\hline & & & & & & \\
\hline & & \multirow{3}{*}{470.56} & \multirow{3}{*}{3.31} & \multirow{3}{*}{6.25} & 3.8 & 207.8 \\
\hline & & & & & & \\
\hline & & & & & 6.1 & 340.3 \\
\hline
\end{tabular}

Fig. 5.16 shows the results with a load capacitor of $1 \mu \mathrm{F}$. It is observed that:

- For a fixed resonant capacitor $C_{r}$, a larger charging current limit $I_{\text {lim }}$ charges load capacitor $C_{o}$ faster. This is because the transferred energy by one charging cycle is higher, and the required number of charging cycles is reduced. 


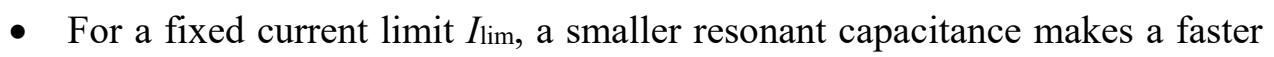
capacitor charging process because of a higher resonant frequency.

- The charging rate $d v_{o} / d t$ decreases with the increase in output voltage. When the output voltage approaches the maximum boost capability (Ilim equals to $I_{H}$ ), the rapid increase in charging time results in more conduction loss. Consequently, the charging efficiency drop rate increases. Take the case that $C_{r}=200.56 \mathrm{nF}$ and $I_{\mathrm{lim}}=4.08 \mathrm{~A}$ as an example. The efficiency is $67.4 \%$ when the load capacitor is charged to $300 \mathrm{~V}$. But it drops greatly to $56.4 \%$ when the load capacitor is charged to $320 \mathrm{~V}$.

- There is an efficiency turning point. For an output voltage lower than the turning point, a smaller charging current limit results in a higher efficiency. For a higher output voltage, a larger charging current limit is preferred in terms of efficiency. Fig. 5.16 demonstrates an example of optimized operation with a resonant capacitance of $470.56 \mathrm{nF}$. The charging current limit can use $3.8 \mathrm{~A}$ for a voltage of lower than $150 \mathrm{~V}$, and $6.1 \mathrm{~A}$ for a higher output voltage.

- In terms of efficiency, a smaller resonant capacitance is preferred. However, the range of charging current is narrower from Fig. 5.14. Hence, there is a tradeoff design considering the efficiency and switching control complexity. 


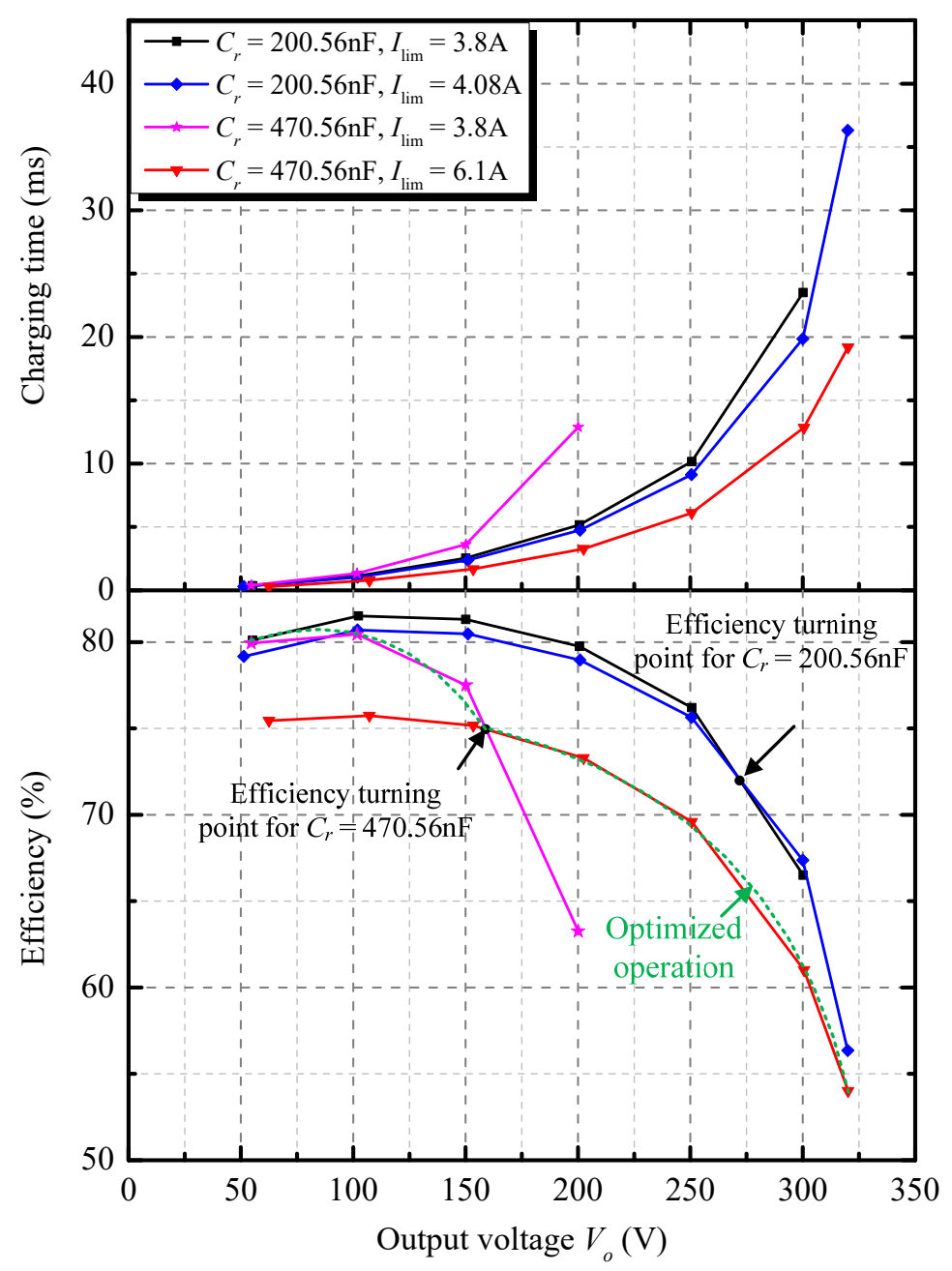

Fig. 5.16 Charging time and efficiency using ZVS with a fixed charging current limit $I_{\text {lim }}$ when load capacitance $C_{o}$ is $1 \mu \mathrm{F}$.

\subsubsection{Hybrid Switching Control}

As discussed in Section 5.2.2, the quasi-flyback converter is operated with hybrid

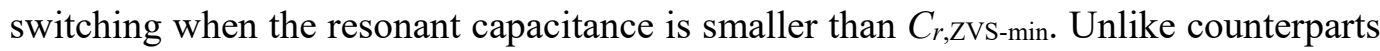
just using the output capacitance of MOSFET as resonant capacitance, this thesis considers the selection of resonant capacitance to minimize switching-off loss. From (5.6), (5.25) and (5.26), the minimum resonant capacitance $C_{r, \mathrm{HY}-\mathrm{min}}$ for hybrid 
switching is $21.2 \mathrm{nF}$ if the load capacitance is $1 \mu \mathrm{F}$. In this study, an input capacitor $C_{\text {in }}$ of $47 \mathrm{nF}$ is added to the prototype circuit to validate the hybrid switching. From the parameters listed in Table 5.1, the lumped resonant capacitance $C_{r}$ is $47.56 \mathrm{nF}$. The charging current limit is fixed in the valley-voltage-switching (VVS) stage. To prevent the energy in leakage inductance being dissipated by the freewheeling diode, the charging current limit $I_{\lim }$ should be smaller than $I_{H}$, which is 1.99 A determined by (5.29). In this experiment, $I_{\lim }$ is set to $1.6 \mathrm{~A}$.

Fig. 5.17 shows the experimental waveforms of first three charging cycles. It is observed that the MOSFET can be turned on at the first valley-voltage. Hence, the switching-on loss is minimized. However, the valley-voltage results in inrush current spikes when the MOSFET is turned on. With the increase in output voltage, the valleyvoltage drops. When zero-voltage is obtained, the switching control is switched to ZVS.

This is demonstrated in Fig. 5.18.

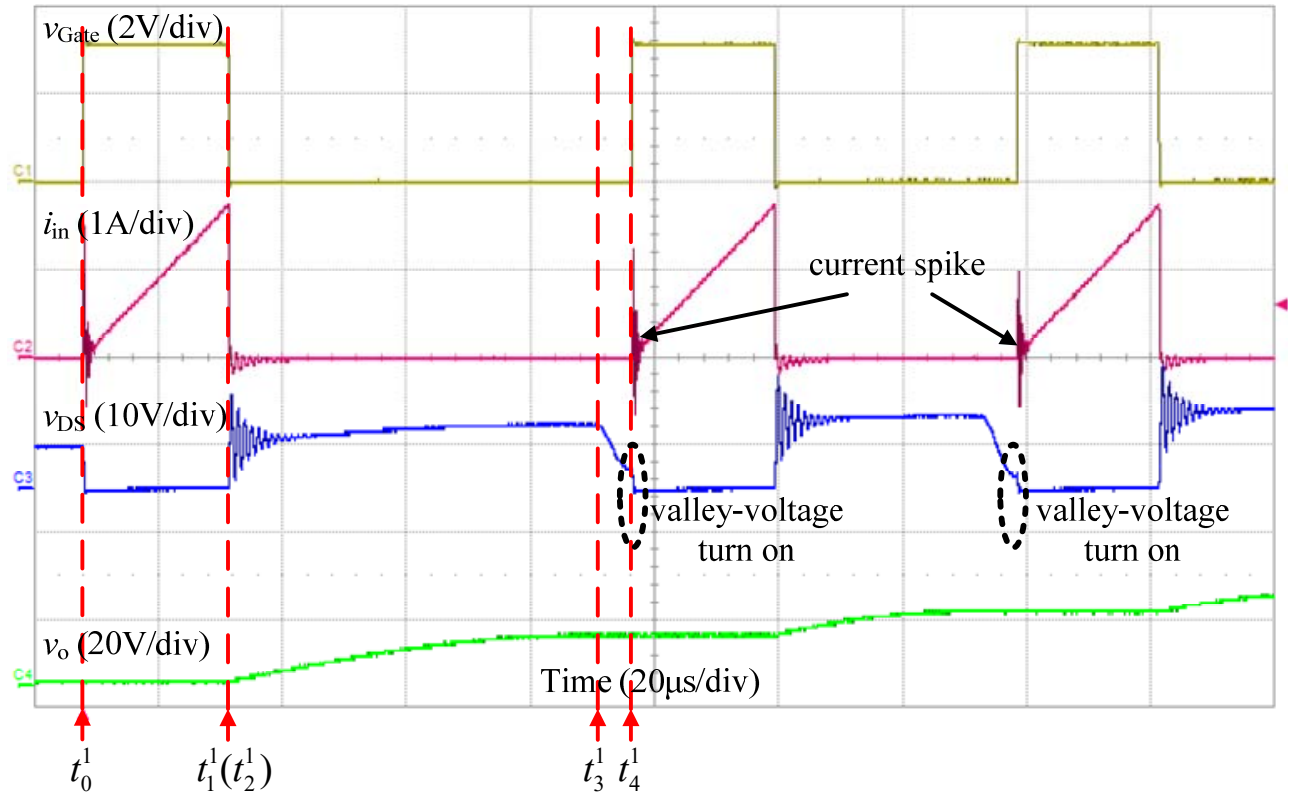

Fig. 5.17 Experimental waveforms of first two charging cycles when $I_{\mathrm{lim}}=1.6 \mathrm{~A}, C_{r}=47.56 \mathrm{nF}$, and $C_{o}=1 \mu \mathrm{F}$. $v_{\text {Gate: }}$ gating signal, $i_{\text {in: }}$ input current, $v_{\mathrm{DS}}$ : voltage across MOSFET, $v_{\mathrm{o}}$ : output voltage. 
For hybrid switching, the output voltage is also constrained by (5.22) if the charging current is fixed. To generate a desired output voltage, the charging current limit $I_{\text {lim }}$ can be adjusted. In this study, $I_{\lim }$ is increased when the switching mode is switched to ZVS. It is assumed that the output voltage is $25 \mathrm{~V}$ when ZVS is obtained. By (5.25), the new charging current limit $I_{\text {lim }}$ should be smaller than 3.98 A. In this experiment, the charging current limit $I_{\text {lim-Vvs }}$ in the VVS mode is 1.6 A while the charging current limit $I_{\text {lim-ZVS }}$ in the ZVS mode is increased to $3.8 \mathrm{~A}$.

Figs. 5.18 and 5.19 show the experimental waveforms. It is observed that there are five charging cycles of VVS. The measured output voltage is about $27 \mathrm{~V}$ when ZVS is obtained. Hence, the current spikes are eliminated from the sixth charging cycle onward. It is also observed that diode $D_{s}$ can be turned off with zero-current under both switching control methods. Hence, the recovery loss can be reduced. As diode $D_{s}$ can be turned on with zero-voltage, the turn-on overshoot voltage is eliminated.

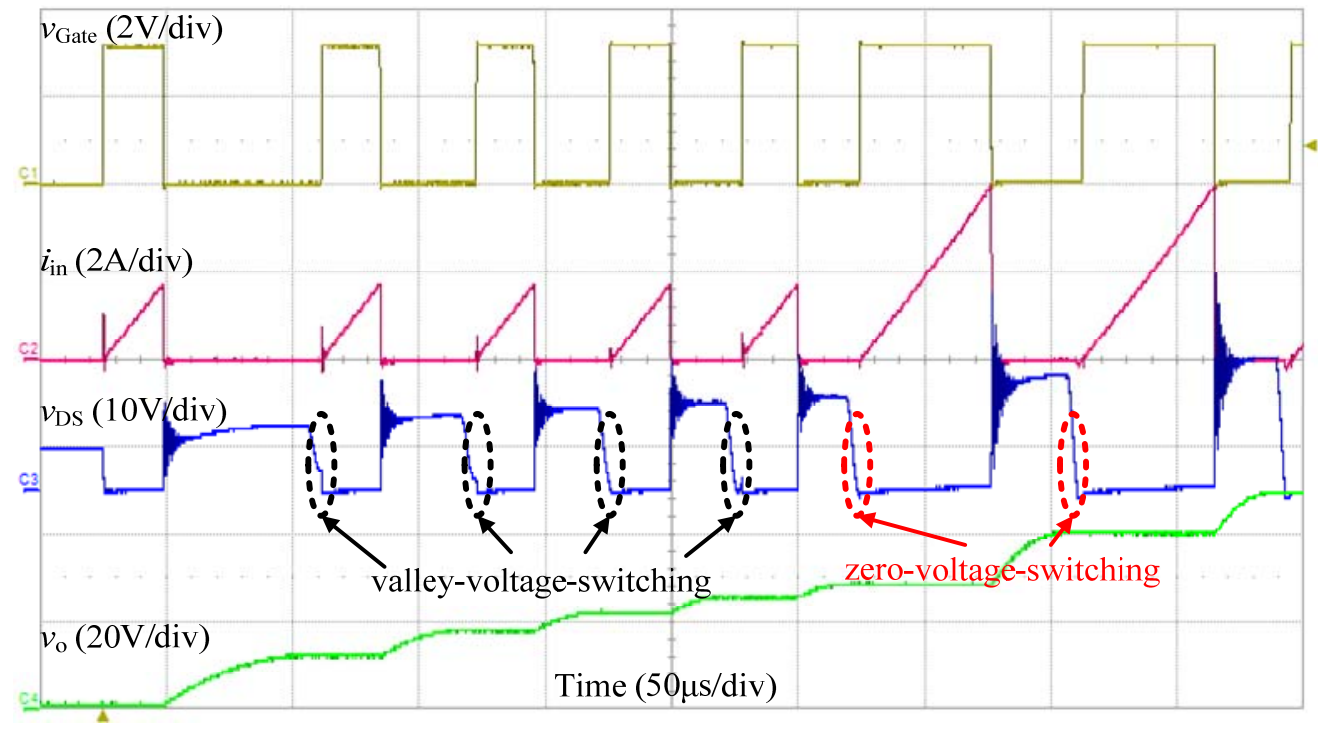

Fig. 5.18 Experimental waveforms of first seven charging cycles when $I_{\text {lim-Vvs }}=1.6 \mathrm{~A}, I_{\mathrm{lim}-\mathrm{ZvS}}=3.8 \mathrm{~A}$, $C_{r}=47.56 \mathrm{nF}$, and $C_{o}=1 \mu \mathrm{F} . v_{\mathrm{Gate}}$ : gating signal, $i_{\mathrm{in}}$ : input current, $v_{\mathrm{DS}}$ : voltage across MOSFET, $v_{\mathrm{o}}$ : output voltage. 


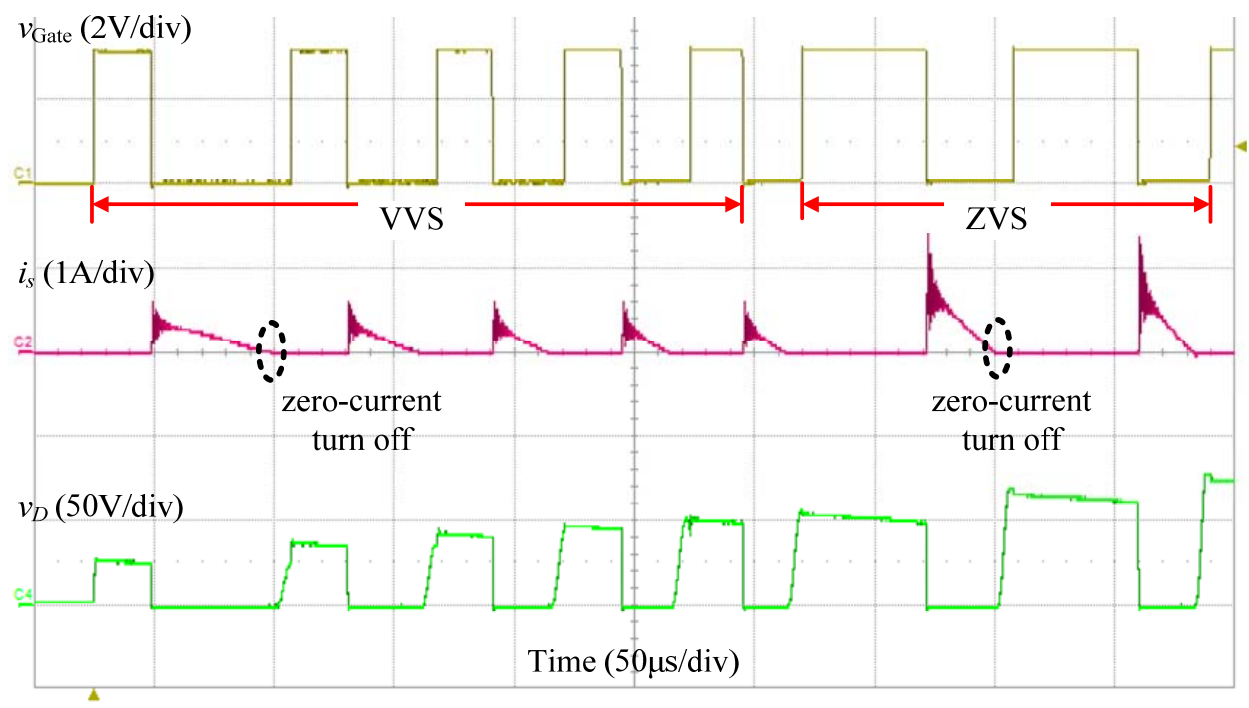

Fig. 5.19 Experimental waveforms of first seven charging cycles when $I_{\text {lim-Vvs }}=1.6 \mathrm{~A}, I_{\text {lim-Zvs }}=3.8$ $\mathrm{A}, C_{r}=47.56 \mathrm{nF}$, and $C_{o}=1 \mu \mathrm{F}$. $v_{\text {Gate }}$ : gating signal, $i_{s}$ : secondary side current, $v_{D}$ : voltage stress on diode $D_{s}$.

With a time division of $20 \mathrm{~ms}$, the current waveform cannot be captured by the current probe. Hence, only the output voltage of charging a load capacitor of $1 \mu \mathrm{F}$ is shown in Fig. 5.20. It is observed that it takes about $100 \mathrm{~ms}$ to charge to $600 \mathrm{~V}$ using hybrid switching approach.

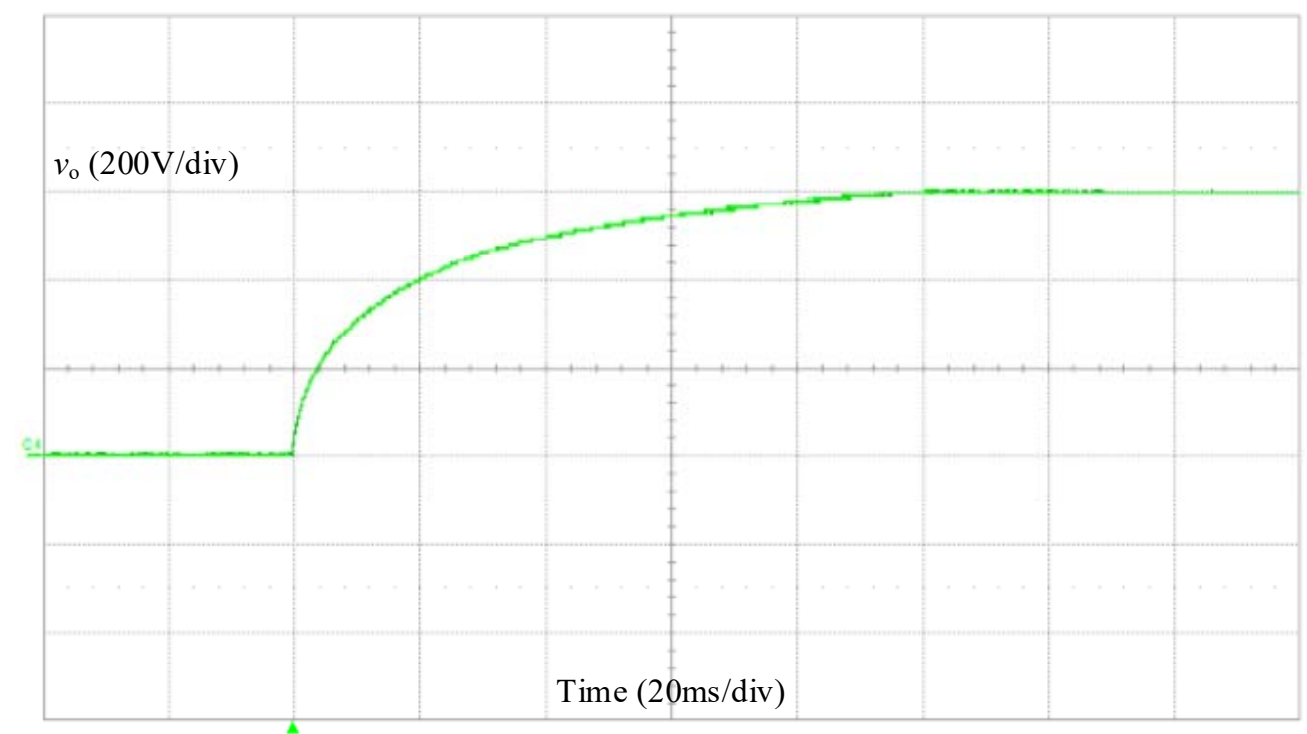

Fig. 5.20 Output voltage $v_{o}$ when $I_{\text {lim-VVS }}=1.6 \mathrm{~A}, I_{\text {lim-ZVS }}=3.8 \mathrm{~A}, C_{r}=47.56 \mathrm{nF}$, and $C_{o}=1 \mu \mathrm{F}$. 
After hybrid switching has been validated, the effect of different resonant capacitance is next studied. In this study, the load capacitance is assumed to be $1 \mu \mathrm{F}$. Two resonant capacitances, $27.56 \mathrm{nF}$ and $47.56 \mathrm{nF}$, are used for this study. The relevant parameters are listed in Table 5.3.

Table 5.3 Parameters for studies on capacitor charging with hybrid switching

\begin{tabular}{|c|c|c|c|c|c|c|}
\hline $\mathrm{C}_{\mathrm{o}}(\mu \mathrm{F})$ & $C_{r, \mathrm{HY}-\min }(\mathrm{nF})$ & $C_{r}(\mathrm{nF})$ & $I_{H}(\mathrm{~A})$ & $I_{\text {lim-Vvs }}(\mathrm{A})$ & $I_{\text {lim-Zvs }}(\mathrm{A})$ & $V_{o, \max }(\mathrm{V})$ \\
\hline \multirow{6}{*}{1} & \multirow{3}{*}{21.6} & \multirow{3}{*}{27.56} & \multirow{3}{*}{1.51} & 1.4 & 1.4 & 315.5 \\
\hline & & & & & & \\
\hline & & & & 1.4 & 3.8 & 853 \\
\hline & \multirow{3}{*}{21.2} & \multirow{3}{*}{47.56} & \multirow{3}{*}{1.99} & 16 & 16 & 2744 \\
\hline & & & & & 1.0 & $2 / 4.4$ \\
\hline & & & & 1.6 & 3.8 & 649.5 \\
\hline
\end{tabular}

Fig. 5.21 shows the PSIM simulation results with a load capacitor of $1 \mu \mathrm{F}$. The observations are similar to those of Fig. 5.16. A smaller resonant capacitance $C_{r}$ results in a shorter charging time and higher charging efficiency. There is also an efficiency turning point which can guide the optimized operation. Take the resonant capacitance of $27.56 \mathrm{nF}$ as an example. The charging current limit is set to $1.4 \mathrm{~A}$ throughout the charging process if the required output voltage is lower than $275 \mathrm{~V}$. For a higher output voltage, the charging current limit can be set to $1.4 \mathrm{~A}$ in the VVS mode, and adjusted to $3.8 \mathrm{~A}$ in the ZVS mode. 


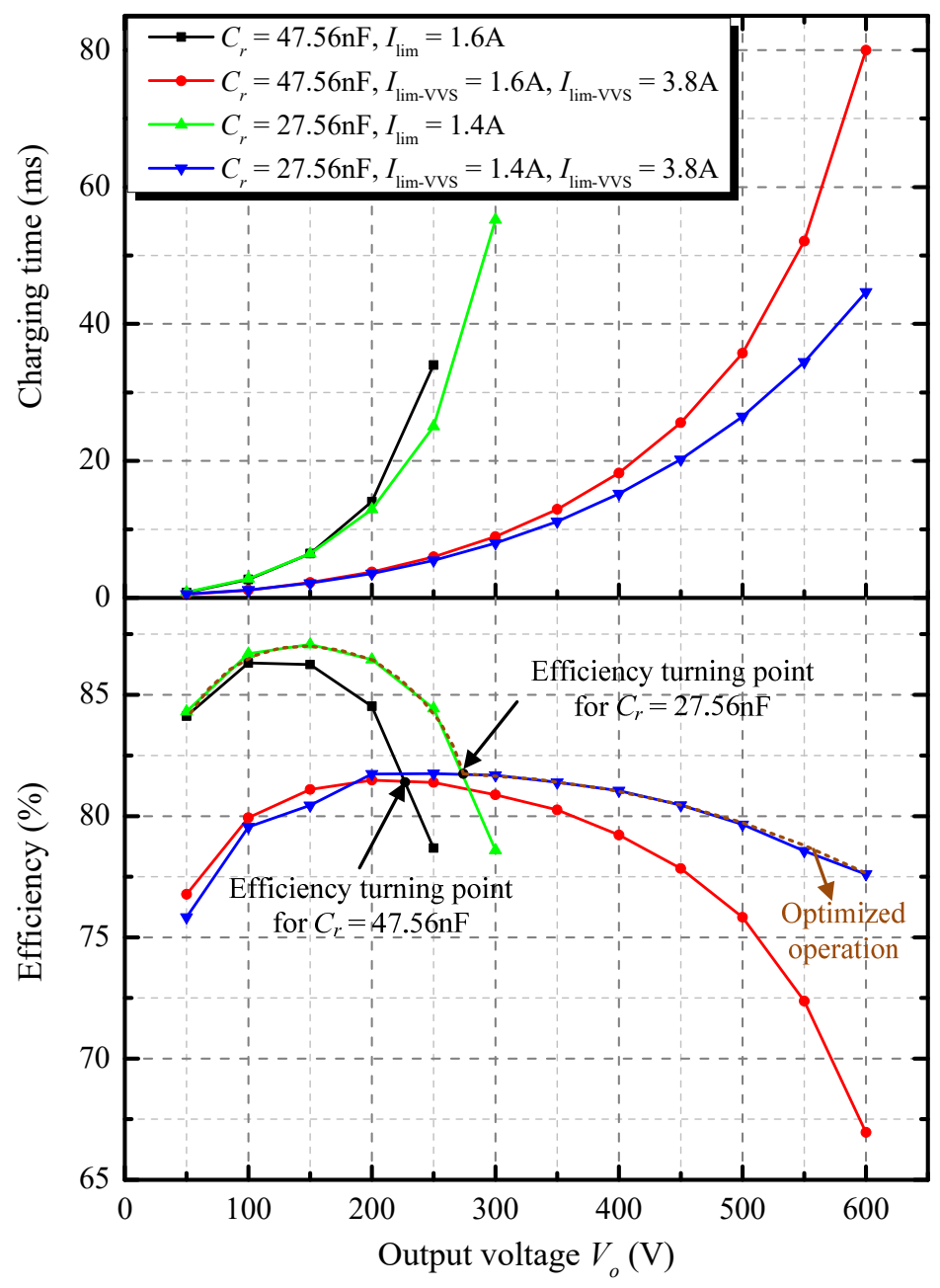

Fig. 5.21 Charging time and efficiency using hybrid switching when load capacitance $C_{o}$ is $1 \mu \mathrm{F}$.

\subsubsection{Switching Control Summary}

The proposed quasi-resonant flyback converter can be used to charge capacitor by ZVS or hybrid switching. This section compares the two switching control methods.

If ZVS can be obtained throughout the capacitor charging process, resonant capacitance $C_{r}$ should be larger than $C_{r, \text { ZVS-min }}$ determined by (5.30). In contrast, the hybrid switching uses both VVS and ZVS if the resonant capacitance is smaller than

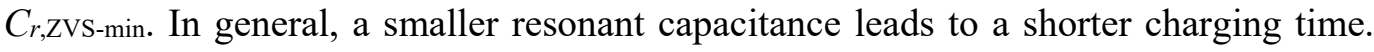


According to (5.6), the hybrid switching control can generate a desired output voltage by a smaller charging current limit. This can reduce the conduction loss. Consequently, hybrid switching is more efficient which can also be observed from Figs. 5.16 and 5.21.

The charging current limits of both switching control methods are constrained by (5.25) to prevent the input current $i_{\text {in }}$ reversing through freewheeling diode $D_{1}$. Consequently, the conduction loss is reduced. Moreover, diode $D_{s}$ in the secondary side can be turned on with zero-voltage, and off with zero-current in both VVS and ZVS modes.

\subsection{Comparisons with Conventional Flyback Converter}

This section presents the comparisons with a conventional flyback converter. As studied in Section 5.4.3, the proposed circuit prefers hybrid switching control to charge capacitor for better efficiency. With a $27.56 \mathrm{nF}$ resonant capacitance, the charging current limits $I_{\mathrm{lim}-\mathrm{VVS}}$ in the VVS mode and $I_{\mathrm{lim}-\mathrm{ZVS}}$ in the ZVS mode are set to $1.4 \mathrm{~A}$ and 3.8 A, respectively. For the conventional flyback converter, the charging limit is set to 3.8 A, and the switching frequency is selected the same as the average switching frequency of the proposed quasi-resonant flyback converter.

\subsubsection{Voltage Stress on Switch}

The voltage stress on MOSFET is

$$
V_{\text {stress }}=V_{\text {in }}+\frac{v_{o}}{n}+V_{\text {osc }}
$$

where $V_{\text {osc }}$ is the amplitude of oscillation voltage caused by the leakage inductance and resonant capacitance. It is determined by

$$
V_{\mathrm{osc}} \approx I_{\lim } \sqrt{\frac{L_{\mathrm{lk}}}{C_{r}}} .
$$


For a quasi-resonant flyback converter, the maximum of $V_{\text {osc }}$ is $12.5 \mathrm{~V}$. In contrast, the maximum of $V_{\text {osc }}$ in a conventional flyback converter is $88 \mathrm{~V}$. Hence, the voltage stress on switch can be reduced using a quasi-resonant technique.

\subsubsection{Charging Time and Efficiency}

Fig. 5.22 shows comparisons on capacitor charging time and efficiency. In general, the proposed quasi-resonant flyback converter requires a longer charging time. For example, the quasi-resonant flyback converter needs $182 \mathrm{~ms}$ to charge a load capacitor of $4 \mu \mathrm{F}$ to $600 \mathrm{~V}$, while the conventional flyback requires less time $(138.3 \mathrm{~ms})$ to complete the charging process. This is because the quasi-resonance does not transfer all the energy stored in the magnetizing inductance to the load capacitor in each charging cycle. However, the quasi-resonance can demagnetize the transformer core to prevent saturation. Hence, a reset clamp circuit is not required.

From Fig. 5.22, it is also observed that the proposed quasi-resonant converter exhibits a better performance in terms of charging efficiency. Compared to a conventional flyback converter, the efficiency is best improved by $10.3 \%$ when a capacitor of $4 \mu \mathrm{F}$ is charged to $150 \mathrm{~V}$. However, the charging rate $d v_{o} / d t$ decreases with the increase in output voltage. This is because the duration of Mode 3 becomes shorter when the load capacitor is charged. As a result, the charging efficiency falls close to a conventional flyback converter when the output voltage approaches the maximum, e.g. $600 \mathrm{~V}$.

When charged to $600 \mathrm{~V}$, the load capacitor of $4 \mu \mathrm{F}$ stores an energy of $0.72 \mathrm{~J}$. If two secondary windings are configured in series, the stored energy and charging time are doubled to $1.44 \mathrm{~J}$ and $364 \mathrm{~ms}$, respectively. Hence, the proposed circuit satisfies the PPT circuit requirements as listed in Table 2.1, i.e. a stored energy of less than $1.5 \mathrm{~J}$ and a pulse frequency of $1 \mathrm{~Hz}$. 


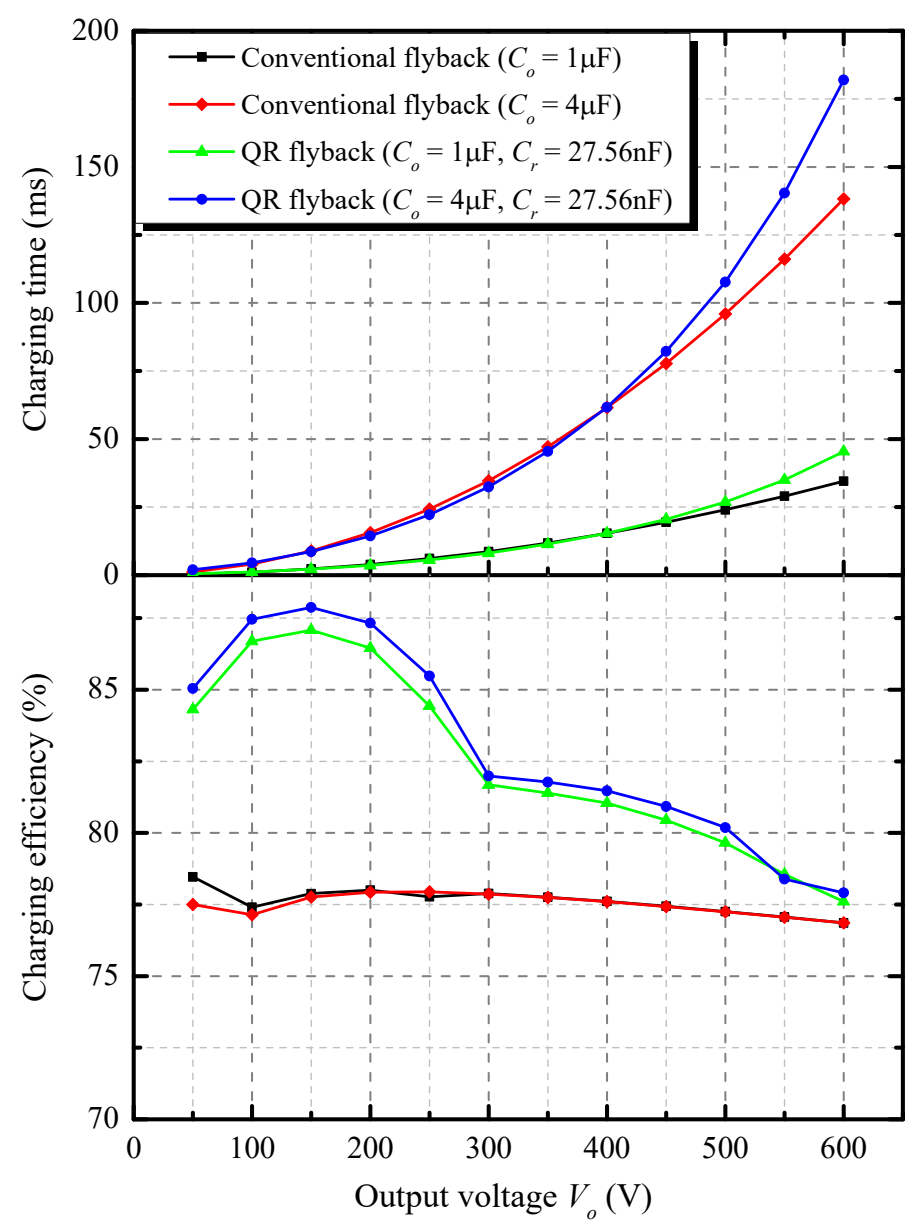

Fig. 5.22 Comparisons with a conventional flyback converter on charging time and efficiency.

Fig. 5.23 shows the energy distribution of the proposed quasi-resonant flyback converter and a convention flyback converter when they charge the load capacitor to 600 V. By the hybrid switching control method, the switching loss $W_{\text {MOSFET_sw }}$ is reduced by more than $3.3 \%$ as compared to the conventional flyback converter. The energy $W_{\mathrm{lk}}$ in leakage inductance of transformer can be recycled due to the quasi-resonant operation. In contrast, the leakage inductance will cause an energy loss of $0.42 \%$ in a conventional flyback converter. Since a smaller charging current limit is used in the VVS mode, the ferrite core loss $W_{\text {TRF_core }}$ is $1.69 \%$ smaller for a load capacitor of $1 \mu \mathrm{F}$, and $2.08 \%$ smaller for a load of $4 \mu \mathrm{F}$. As the recovery loss of the diode in the secondary side is 
eliminated, its energy loss $W_{\text {Diode }}$ is $0.8 \%$ smaller than the conventional flyback converter. In the aforementioned description, the proposed quasi-resonant flyback converter needs a longer charging time. Consequently, it has higher MOSFET

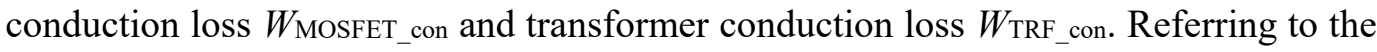

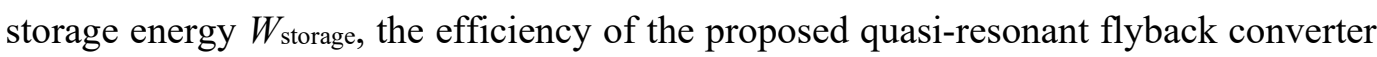
is $0.71 \%$ higher for a load capacitor of $1 \mu \mathrm{F}$, and $1.05 \%$ higher for a load capacitor of 4 $\mu \mathrm{F}$. In terms of efficiency, a quasi-resonant flyback behaves better than a conventional flyback converter for capacitor charging.

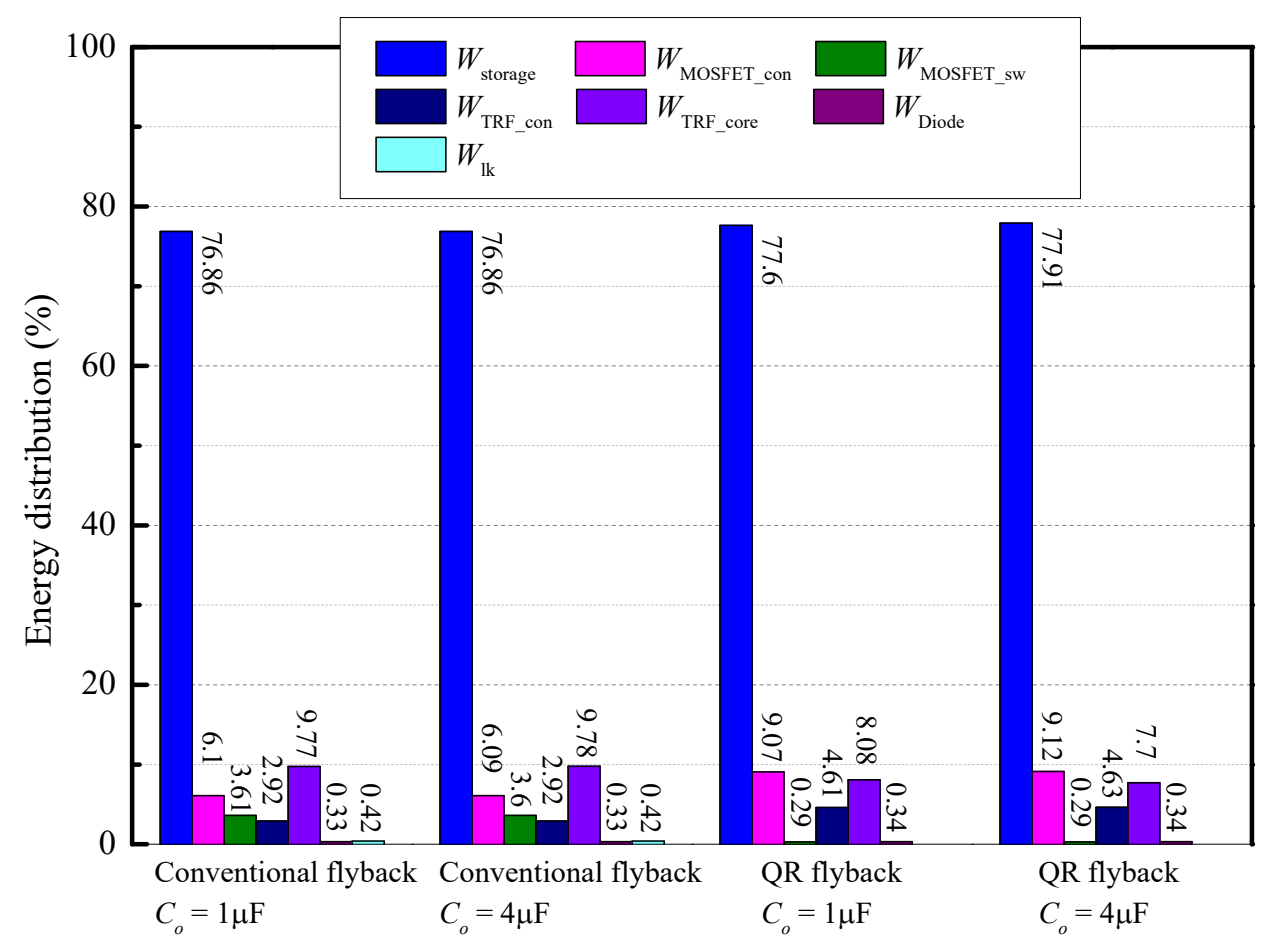

Fig. 5.23 Energy distribution of quasi-resonant flyback converter and conventional flyback converter when the load capacitors are charged to $600 \mathrm{~V}$. 


\subsection{Summary}

In this chapter, a quasi-resonant flyback converter is proposed for capacitor charging. Depending on the operating conditions, the circuit can be operated with zero-voltageswitching (ZVS) and hybrid switching.

If the proposed topology charges a load capacitor with ZVS mode during the entire

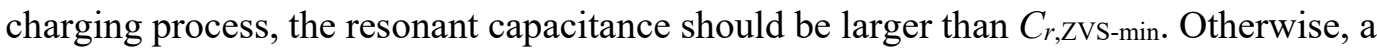
hybrid switching approach is used, i.e. valley-voltage-switching (VVS) in the first few charging cycles and ZVS in the remaining charging cycles. Both VVS and ZVS can minimize switching loss. Due to the quasi-resonant operation, the diode in the secondary side can be turned on with zero-voltage, and off with zero-current. Hence, the turn-on overshoot voltage and recovery loss can be reduced.

The experiment has been conducted to validate the proposed capacitor charging method by a quasi-resonant flyback converter. The study reveals that the hybrid switching control is preferred. When compared to a conventional flyback converter, the prototype circuit exhibits a better charging efficiency when the output voltage is lower than $600 \mathrm{~V}$. 


\section{Chapter 6}

\section{Conclusion and Future Work}

\subsection{Conclusion}

In this thesis, various high voltage DC-DC converters have been proposed for the pulsed plasma thruster (PPT) applications.

A flyback circuit has been proposed for a PPT igniter. Unlike other topologies that require physical high voltage capacitors, this topology uses the parasitic capacitances of transformer, MOSFET, diode and igniter spark plugs. Hence, the reliability of PPT igniter is improved by avoiding the capacitor failure probability of $13.4 \%$.

While the flyback circuit can be realized with a reduced number of components, the hard-switching deteriorates the efficiency, e.g. $38.1 \%$ at an output voltage of $1.88 \mathrm{kV}$, and even worse at a higher output voltage.

To overcome the drawbacks of hard-switching technique, this thesis also proposes a quasi-resonant DC-DC converter for a PPT igniter. The resonant tank can be driven by a single switch to operate in quasi-resonant state. With the implementation of a single switch, the switching control can be realized without a dead-time compared to a switch bridge. Moreover, the switching frequency is extended to higher than half of the natural resonant frequency. Hence, it has a better boost capability than other resonant converters operating with zero-current-switching (ZCS). For example, the maximum normalized voltage gain of a parallel resonant converter (PRC) is 1.33 while the prototype circuit can achieve 6.25. With the ZCS operation and a single switch implementation, the switch 
conduction loss is reduced, and the efficiency is improved compared to an LLC resonant converter (LLC-RC). Take the case that the normalized voltage gain is 3.375 as an example. The switch conduction loss and efficiency of proposed circuit are $27.62 \mathrm{~mW}$ and $47.27 \%$, respectively. In contrast, the switch conduction loss and efficiency of LLC$\mathrm{RC}$ are $240.48 \mathrm{~mW}$ and $35.22 \%$, respectively.

Both simulation and experimental studies have validated that the flyback circuit and single switch quasi-resonant DC-DC converter could meet the requirements of a PPT igniter, and trigger the plasma generation successfully in vacuum environment.

In this thesis, a quasi-resonant flyback converter is proposed for capacitor charging. Two switching control approaches have been discussed. One is zero-voltage-switching (ZVS) used throughout the charging process. The other one is hybrid switching including valley-voltage-switching (VVS) in the first few charging cycles and ZVS in the remaining cycles. Using ZVS throughout the charging process, a larger resonant capacitance is required, resulting in longer charging time and limited output voltage. Hence, VVS is preferable. Both VVS and ZVS can minimize switching-on loss compared to a hard-switching technique. Moreover, switching-off loss can be minimized by selecting a proper resonant capacitance. The energy in transformer leakage inductance can be recycled. Thereby, the energy loss is reduced. Compared to the conventional flyback converter, the capacitor charging efficiency can be best improved by $10.3 \%$ when the output voltage is $150 \mathrm{~V}$. However, the charging time increases rapidly when the output voltage approaches the maximum. This leads to a significant conduction loss and efficiency deterioration. 


\subsection{Future Work}

The following suggestions are works to be explored in the future.

From the literature review, the power requirement of PPT igniter has not been specified clearly so far. The future work should cover the study on how the power of PPT igniter affects the system performance such as reliability, efficiency, etc. This can guide the design of PPT igniter circuit.

As reviewed in Chapter 2, the normalized voltage gain of resonant converters using a switch bridge can be analyzed by the first harmonic approach (FHA). However, the results of FHA become less accurate if the resonant converters are operated with discontinuous-current-mode (DCM). Thereby, detailed studies have been conducted on LCC-RC and LLC-RC taking the behavior of the rectifier circuit into consideration. As for the single switch quasi-resonant DC-DC converter proposed in Chapter 4, the normalized voltage gain is obtained by numerical method and an approximated AC equivalent circuit. In future work, a diode bridge could be employed to simplify the analysis on the rectifier behavior instead of a voltage multiplier. Using the complete AC equivalent circuit and considering the states of diodes, more accurate details can be determined such as normalized voltage gain, rang of switching frequency, output power, etc. Moreover, it is helpful for the optimization to obtain a better efficiency.

In Chapter 5, a quasi-resonant flyback converter is proposed for capacitor charging. It is preferable to operate with hybrid switching. The charging current limit is adjusted to achieve a desired output voltage when the switching control mode is switched to ZVS. However, this does not cover optimization. The future work is to investigate more on the energy loss model, and determine the selection of charging current limits for VVS and ZVS modes. 
Lastly, new prototype circuits can be fabricated with the integration of proposed PPT igniter circuit and capacitor charging circuit. The experiment will be conducted in vacuum environment to measure the performance including the efficiency, EMI noise, reliability, etc. 


\section{Appendix A}

The two matrixes $\boldsymbol{U}$ and $\boldsymbol{I}$ are defined as

$$
\dot{I}=\left[\begin{array}{c}
\dot{I}_{1} \\
\dot{I}_{2}
\end{array}\right], \dot{U}=\left[\begin{array}{c}
\dot{U}_{1} \\
\dot{U}_{2}
\end{array}\right]
$$

The П-type two-port circuit network shown in Fig. A1(a) can be represented by

$$
\dot{I}=Y \dot{U}
$$

where $\boldsymbol{Y}$ is defined as

$$
Y=\left[\begin{array}{ll}
y_{11} & y_{12} \\
y_{21} & y_{22}
\end{array}\right]=\left[\begin{array}{cc}
Y_{1}+Y_{2} & -Y_{2} \\
-Y_{2} & Y_{2}+Y_{3}
\end{array}\right]
$$

$\mathrm{Y}_{1}, \mathrm{Y}_{2}$ and $\mathrm{Y}_{3}$ are the admittances of $C_{\mathrm{in}}, L_{\mathrm{lk}}$ and $C_{w}$, respectively.

(A1) can be transformed to

$$
\dot{U}=Z \dot{I}
$$

where $\boldsymbol{Z}$ is defined as

$$
Z=\left[\begin{array}{ll}
z_{11} & z_{12} \\
z_{21} & z_{22}
\end{array}\right]=\left[\begin{array}{cc}
Z_{1}+Z_{2} & Z_{2} \\
Z_{2} & Z_{2}+Z_{3}
\end{array}\right]
$$

$\mathrm{Z}_{1}, \mathrm{Z}_{2}$ and $\mathrm{Z}_{3}$ are the impedances of components in T-type two-port circuit network, $L_{1}$, $C_{T}$ and $L_{2}$ as shown in Fig. A1(b). 


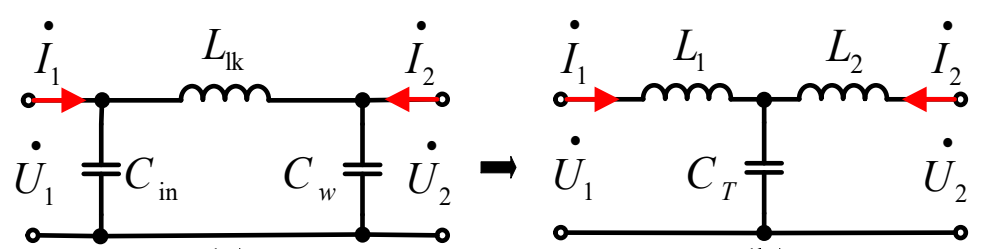

(a)

(b)

Fig. A1 Two-port circuit network: (a) П-type, and (b) T-type.

The $\mathbf{Z}$ can be calculated by

$$
z_{11}=\frac{y_{22}}{\Delta y}, z_{12}=-\frac{y_{12}}{\Delta y}, z_{21}=-\frac{y_{21}}{\Delta y}, z_{22}=\frac{y_{11}}{\Delta y}
$$

where $\Delta y=y_{11} y_{22}-y_{12} y_{21}$.

By (A1) - (A5), the impedances are given as:

$$
\left\{\begin{array}{l}
Z_{1}=\frac{s L_{\mathrm{lk}} C_{w}}{s^{2} C_{\mathrm{in}} C_{w} L_{\mathrm{lk}}+\left(C_{\mathrm{in}}+C_{w}\right)} \\
Z_{2}=\frac{1}{s^{3} C_{\mathrm{in}} C_{w} L_{\mathrm{lk}}+s\left(C_{\mathrm{in}}+C_{w}\right)} \\
Z_{3}=\frac{s L_{\mathrm{lk}} C_{\mathrm{in}}}{s^{2} C_{\mathrm{in}} C_{w} L_{\mathrm{lk}}+\left(C_{\mathrm{in}}+C_{w}\right)}
\end{array}\right.
$$

The switching frequency is smaller than the resonant frequency occurring among $C_{\mathrm{in}}$, $C_{w}$ and $L_{\mathrm{k}}$ because of inductor $L_{\mathrm{in}}$. Hence, $\mathrm{Z}_{1}$ and $\mathrm{Z}_{3}$ are the impedances of inductors while $Z_{2}$ is the impedance of capacitor. The components' values in Fig. A1(b) are

$$
\left\{\begin{array}{l}
L_{1}=\frac{L_{\mathrm{lk}}}{1+\frac{C_{\mathrm{in}}}{C_{w}}-\omega^{2} L_{\mathrm{lk}} C_{\mathrm{in}}} \\
C_{T}=-\omega^{2} L_{\mathrm{lk}} C_{\mathrm{in}} C_{w}+C_{\mathrm{in}}+C_{w} \\
L_{2}=\frac{L_{\mathrm{lk}}}{1+\frac{C_{w}}{C_{\mathrm{in}}}-\omega^{2} L_{\mathrm{lk}} C_{w}}
\end{array}\right.
$$

where $\omega$ is the switching angular frequency.

The lumped series inductance $L_{s}$ is given by 


$$
L_{s}=L_{\text {in }}+L_{1} \text {. }
$$

The impedance of $L_{2}, L_{m}$ and $R_{\mathrm{ac}}$ in Fig. 4.4(b) is

$$
Z_{4}=\frac{R_{\mathrm{ac}}}{1+\left(\frac{R_{\mathrm{ac}}}{\omega L_{m}}\right)^{2}}+j \omega\left(L_{2}+\frac{L_{m}}{1+\left(\frac{\omega L_{m}}{R_{\mathrm{ac}}}\right)^{2}}\right)=a+j \omega b
$$

while the impedance of $L_{T}$ and $R_{T}$ in Fig. 4.4(c) is

$$
Z_{5}=\frac{R_{T}}{1+\left(\frac{R_{T}}{\omega L_{T}}\right)^{2}}+j \omega \frac{L_{T}}{1+\left(\frac{\omega L_{T}}{R_{T}}\right)^{2}} .
$$

By solving $Z_{4}=Z_{5}$, the values of $R_{T}$ and $L_{T}$ can be obtained with a given switching angular frequency $\omega$ :

$$
\begin{aligned}
& L_{T}=b+\frac{1}{b}\left(\frac{a}{\omega}\right)^{2}, \\
& R_{T}=\frac{a^{2}+(\omega b)^{2}}{a} .
\end{aligned}
$$

The amplitude ratio of $v_{p}$ and $v_{T}$ is

$$
\left|\frac{v_{p}}{v_{T}}\right|=\frac{1}{\sqrt{\left(1+\frac{L_{2}}{L_{m}}\right)^{2}+\left(\frac{\omega L_{2}}{R_{\mathrm{ac}}}\right)^{2}}} .
$$




\section{List of Publications}

1 B. X. Li, K. S. Low, and B. Kang, "An accurate lossless current sensing approach for a DC-DC converter with online calibration," in Proc. IEEE PES Asia-Pacific Power Energy Eng. Conf. (APPEEC), 2014, pp. 1-5.

2 B. Kang and K. S. Low, "A single-switch high voltage quasi-resonant DC-DC converter for a pulsed plasma thruster," in Proc. IEEE 11th Int. Conf. Power Electron. Drive Sys. (PEDS), 2015, pp. 509-513.

3 B. Kang, K. S. Low, J. Soon, and Q. V. Tran, "Single switch quasi-resonant DCDC converter for a pulsed plasma thruster of satellites," IEEE Trans. Power Electron., vol. vol. 32, no. 6, pp. 4503-4513, 2017.

4 B. X. Li, K. S. Low, B. Kang, "Modeling and online health monitoring of interleaved DC-DC converters using biogeographical based optimization with grouped immigration", submitted to IEEE Trans. Power Electron.

5 B. Kang, K. S. Low, J. Soon, and Q. V. Tran, “Quasi-resonant flyback converter using efficient switching control for capacitor charging", submitted to IEEE Trans. Power Electron. 


\section{Bibliography}

[1] K. H. Cheah and K. S. Low, "Fabrication and performance evaluation of a high temperature co-fired ceramic vaporizing liquid microthruster," J. Micromech. Microeng., vol. 25, no. 1, p. 015013, 2015.

[2] S. Xie, G. X. Lee, K. S. Low, and E. Gunawan, "Wireless sensor network for satellite applications: a survey and case study," Unmanned Systems, vol. 02, no. 03, pp. 261-277, Jul 2014.

[3] M. Guyot et al., "Electrostatic propulsion for satellites application to the Robusta3 nanosatellite," IEEE Trans. Dielectr. Electr. Insul., vol. 21, no. 3, pp. 1161-1165, Jun. 2014.

[4] T. Yi, Y. Yan, S. Wen, and Y. Zhou, "Analytical solution of a super low satellite orbit disturbed by atmospheric drag considering J2 perturbation," in Proc. 5th Int. Conf. Intelligent Human-Machine Systems and Cybernetics (IHMSC), 2013, vol. 2, pp. 320-325.

[5] M. Coletti, S. Ciaralli, and S. B. Gabriel, "PPT development for nanosatellite applications: experimental results," IEEE Trans. Plasma Sci., vol. 43, no. 1, pp. 218-225, Jan. 2015.

[6] M. D. Pham, K. S. Low, S. T. Goh, and S. Chen, "Gain-scheduled extended kalman filter for nanosatellite attitude determination system," IEEE Trans. Aerosp. Electron. Syst., vol. 51, no. 2, pp. 1017-1028, Apr. 2015.

[7] R. Vasantharaj, D. Sureshbabu, and D. Kumar, "Advanced pulsed plasma thruster for satellites orbit control," in Proc. Recent Advances in Space Technology Services and Climate Change (RSTSCC), 2010, 2010, pp. 111-113.

[8] R. L. Sackheim and D. C. Byers, "Status and issues related to in-space propulsion systems," J. Propul. Power, vol. 14, no. 5, pp. 669-675, Sep. 1998.

[9] M. Martinez-Sanchez and J. E. Pollard, "Spacecraft electric propulsion-an overview," J. Propul. Power, vol. 14, no. 5, pp. 688-699, Sep. 1998.

[10] R. L. Burton and P. J. Turchi, "Pulsed plasma thruster," J. Propul. Power, vol. 14, no. 5, pp. 716-735, Sep. - Oct. 1998.

[11] Z. Chuck, B. Scott, S. Paul, and H. Andrew, "On-orbit testing of the EO-1 pulsed plasma thruster," in 38th AIAA/ASME/SAE/ASEE Joint Propulsion Conference \& Exhibit(Joint Propulsion Conferences: American Institute of Aeronautics and Astronautics, 2002.

[12] C. D. Rayburn, M. E. Campbell, and A. T. Mattick, "Pulsed plasma thruster system for microsatellites," J. Spacecraft Rockets, vol. 42, no. 1, pp. 161-170, Jan.-Feb. 2005.

[13] M. Tanaka, S. Kisaki, T. Ikeda, and H. Tahara, "Research and development of pulsed plasma thruster systems for nano-satellites at Osaka Institute of Technology," in Proc. IEEE Vehicle Power and Propul. Conf. (VPPC), 2012, pp. 517-522.

[14] M. Coletti, F. Guarducci, and S. B. Gabriel, "A micro PPT for cubesat application: design and preliminary experimental results," Acta Astronautica, vol. 69, no. 3-4, pp. 200-208, Apr. 2011.

[15] M. Rezanejad, A. Sheikholeslami, and J. Adabi, "High-voltage modular switched capacitor pulsed power generator," IEEE Trans. Plasma Sci., vol. 42, no. 5, pp. 1373-1379, May 2014.

[16] G. Herdrich et al., "Advanced plasma (propulsion) concepts at IRS," Vacuum, vol. 88, pp. 36-41, Feb. 2013.

[17] T. Schonherr, K. Komurasaki, F. Romano, B. Massuti-Ballester, and G. Herdrich, "Analysis of atmosphere-breathing electric propulsion," IEEE Trans. Plasma Sci., vol. 43, no. 1, pp. 287-294, Jan. 2015. 
[18] A. Nawaz, M. Lau, G. Herdrich, and M. Auweter-Kurtz, "Investigation of the magnetic field in a pulsed plasma thruster," J. AIAA, vol. 46, no. 11, pp. 2881-2889, Nov. 2008.

[19] S. Yang, A. Bryant, P. Mawby, D. Xiang, L. Ran, and P. Tavner, "An industrybased survey of reliability in power electronic converters," IEEE Trans. Ind. Appl., vol. 47, no. 3, pp. 1441-1451, May/Jun. 2011.

[20] S. Ciaralli, M. Coletti, and S. B. Gabriel, "Performance and lifetime testing of a pulsed plasma thruster for Cubesat applications," Aerosp. Sci. Technol., vol. 47, pp. 291-298, Dec. 2015.

[21] G.-H. Shin, G.-S. Shin, M.-R. Nam, K.-I. Kang, and J.-T. Lim, "High voltage DCDC converter of pulsed plasma thruster for science and technology satellite-2 (STSAT-2)," in Proc. PEDS Int. Conf. Power Electron. and Drives Syst., 2005, vol. 2, pp. 926-931.

[22] M. Compadre, C. Clark, P. Marinov, S. Ciaralli, M. Coletti, and S. Gabriel, "Power efficient pulsed plasma thruster with precise control of high voltage generation," 2013.

[23] T. Huang, Z. Wu, X. Liu, K. Xie, N. Wang, and Y. Cheng, "Study of breakdown in an ablative pulsed plasma thruster," Phys. Plasmas, vol. 22, no. 10, p. 103511, Oct. 2015.

[24] W. J. Guman and P. E. Peko, "Solid-propellant pulsed plasma microthruster studies," J. Spacecraft Rockets, vol. 5, no. 6, pp. 732-733, Jun. 1968.

[25] S. Scharring, S. Karg, R.-A. Lorbeer, N. Dahms, and H.-A. Eckel, "Low-noise thrust generation by laser-ablative micropropulsion," in Proc. Int. Electr. Propul. Conf. (IEPC), 2015.

[26] R. Zhang, D. Zhang, F. Zhang, Z. He, and J. Wu, "Deposition of fluorocarbon films by pulsed plasma thruster on the anode side," Appl. Surf. Sci., vol. 270, no. 0, pp. 352-358, Jan. 2013.

[27] A. A. Fardoun and E. H. Ismail, "Ultra step-up DC-DC converter with reduced switch stress," IEEE Trans. Ind. Applicat. , vol. 46, no. 5, pp. 2025-2034, Sep/Oct. 2010.

[28] F. L. Luo and H. Ye, "Ultra-lift Luo-converter," Proc IEE Electr. Power Appl., vol. 152, no. 1, pp. 27-32, Sep. 2005.

[29] J. C. Rosas-Caro, F. Mancilla-David, J. C. Mayo-Maldonado, J. M. GonzalezLopez, H. L. Torres-Espinosa, and J. E. Valdez-Resendiz, "A transformer-less highgain boost converter with input current ripple cancelation at a selectable duty cycle," IEEE Trans. Ind.l Electron., vol. 60, no. 10, pp. 4492-4499, Oct. 2013.

[30] K. I. Hwu, C. F. Chuang, and W. C. Tu, "High voltage-boosting converters based on bootstrap capacitors and boost inductors," IEEE Trans. Ind. Electron., vol. 60, no. 6, pp. 2178-2193, Jun. 2013.

[31] W. Li and X. He, "Review of nonisolated high-step-up DC/DC converters in photovoltaic grid-connected applications," IEEE Trans. Ind. Electron., vol. 58, no. 4, pp. 1239-1250, Apr. 2011.

[32] M. Rezanejad, A. Sheikholeslami, and J. Adabi, "Modular switched capacitor voltage multiplier topology for pulsed power supply," IEEE Trans. Dielectr. Electr. Insul., vol. 21, no. 2, pp. 635-643, Apr. 2014.

[33] S. Zabihi, Z. Zabihi, and F. Zare, "A solid-state Marx generator with a novel configuration," IEEE Trans. Plasma Sci., vol. 39, no. 8, pp. 1721-1728, Aug. 2011.

[34] T. Sakamoto, A. Nami, M. Akiyama, and H. Akiyama, "A repetitive solid state Marx-type pulsed power generator using multistage switch-capacitor cells," IEEE Trans. Plasma Sci., vol. 40, no. 10, pp. 2316-2321, Oct. 2012.

[35] T. Sakamoto and H. Akiyama, "Solid-state dual Marx generator with a short pulsewidth," IEEE Trans. Plasma Sci., vol. 41, no. 10, pp. 2649-2653, Oct. 2013.

[36] J. Sun, X. Ding, M. Nakaoka, and H. Takano, "Series resonant ZCS-PFM DC-DC converter with multistage rectified voltage multiplier and dual-mode PFM control 
scheme for medical-use high-voltage X-ray power generator," IEE Electric Power Appl., vol. 147, no. 6, pp. 527-534, Nov. 2000.

[37] C. M. Young, M. H. Chen, T. A. Chang, C. C. Ko, and K. K. Jen, "Cascade Cockcroft - Walton voltage multiplier applied to transfomerless high step-up DCDC converter," IEEE Trans. Ind. Electron., vol. 60, no. 2, pp. 523-537, Feb. 2013.

[38] M. Prudente, L. L. Pfitscher, G. Emmendoerfer, E. F. Romaneli, and R. Gules, "Voltage multiplier cells applied to non-isolated DC-DC converters," IEEE Trans. Power Electron., vol. 23, no. 2, pp. 871-887, Mar. 2008.

[39] L. Müller and J. W. Kimball, "High gain DC-DC converter based on the CockcroftWalton multiplier," IEEE Trans. Power Electron., vol. 31, no. 9, pp. 6405-6415, Sep. 2016.

[40] B. Axelrod, Y. Berkovich, A. Shenkman, and G. Golan, "Diode-capacitor voltage multipliers combined with boost-converters: topologies and characteristics," IET Power Electron., vol. 5, no. 6, pp. 873-884, Mar. 2012.

[41] S. Zabihi, F. Zare, G. Ledwich, A. Ghosh, and H. Akiyama, "A novel high-voltage pulsed-power supply based on low-voltage switch-capacitor units," IEEE Trans. Plasma Sci., vol. 38, no. 10, pp. 2877-2887, Oct. 2010.

[42] Z. Salam, M. Facta, and M. Amjad, "Dielectric barrier discharge ozonizer using the transformerless single-switch resonant converter for portable applications," IEEE Trans. Ind. Appl., vol. 50, no. 3, pp. 2197-2206, May/Jun. 2014.

[43] M. Amjad, Z. Salam, M. Facta, and S. Mekhilef, "Analysis and implementation of transformerless LCL resonant power supply for ozone generation," IEEE Trans. Power Electron., vol. 28, no. 2, pp. 650-660, Feb. 2013.

[44] X. Bonnin, J. Brandelero, N. Videau, H. Piquet, and T. Meynard, "A high voltage high frequency resonant inverter for supplying DBD devices with short discharge current pulses," IEEE Trans. Power Electron., vol. 29, no. 8, pp. 4261-4269, Aug. 2014.

[45] M. Pavlovsky, S. W. H. d. Haan, and J. A. Ferreira, "Reaching high power density in multikilowatt DC-DC converters with galvanic isolation," IEEE Trans. Power Electron., vol. 24, no. 3, pp. 603-612, Mar. 2009.

[46] T. Sakamoto, T. Tanabe, S. Katsuki, and H. Akiyama, "Solid propellant pulsed plasma thruster driven by magnetic pulse compression circuit," in Proc. IEEE 19th Pulsed Power Conference (PPC), 2013, pp. 1-5.

[47] P. Davari, F. Zare, A. Ghosh, and H. Akiyama, "High-voltage modular power supply using parallel and series configurations of flyback converter for pulsed power applications," IEEE Trans. Plasma Sci., vol. 40, no. 10, pp. 2578-2587, Oct. 2012.

[48] J. M. Burkhart, R. Korsunsky, and D. J. Perreault, "Design methodology for a very high frequency resonant boost converter," IEEE Trans. Power Electron., vol. 28, no. 4, pp. 1929-1937, Apr. 2013.

[49] R. C. N. Pilawa-Podgurski, A. D. Sagneri, J. M. Rivas, D. I. Anderson, and D. J. Perreault, "Very-high-frequency resonant boost converters," IEEE Trans. Power Electron., vol. 24, no. 6, pp. 1654-1665, Jun. 2009.

[50] A. Santoja, A. Barrado, C. Fernandez, M. Sanz, C. Raga, and A. Lazaro, "High voltage gain DC-DC converter for micro and nanosatellite electric thrusters," in Proc. IEEE 28th Ann. Appl. Power Electron. Conf. and Exposition (APEC), 2013, pp. 2057-2063.

[51] A. K. Singh, P. Das, and S. K. Panda, "High voltage high frequency resonant DCDC converter for electric propulsion for micro and nanosatellites," in Proc. IEEE 36th Int. Telecommun. Energy Conf. (INTELEC), 2014, pp. 1-5.

[52] R. L. Steigerwald, "A comparison of half-bridge resonant converter topologies," IEEE Trans. Power Electron., vol. 3, no. 2, pp. 174-182, Apr. 1988.

[53] M. Salem, A. Jusoh, N. R. N. Idris, and I. Alhamrouni, "Performance study of series resonant converter using zero voltage switching," in Proc. IEEE Conf. Energy Convers. (CENCON), 2014, pp. 96-100. 
[54] G. Ivensky, I. Zeltser, A. Kats, and S. Ben-Yaakov, "Reducing IGBT losses in ZCS series resonant converters," IEEE Trans. Ind. Electron., vol. 46, no. 1, pp. 67-74, Feb. 1999.

[55] S. S. Lee, S. Iqbal, and M. Kamarol, "Control of ZCS-SR inverter-fed voltage multiplier-based high-voltage DC-DC converter by digitally tuning tank capacitance and slightly varying pulse frequency," IEEE Trans. Power Electron., vol. 27, no. 3, pp. 1076-1083, Mar. 2012.

[56] L. Sze Sing, S. Iqbal, and M. K. Mohd Jamil, "A new ZCS series resonant highvoltage DC power supply," in Proc. IEEE Int. Conf. Power and Energy (PECon), 2012, pp. 44-48.

[57] T. N. Gücin, M. Biberoğlu, and B. Fincan, "A constant-current constant-voltage charging based control and design approach for the parallel resonant converter," in Proc. International Conference on Research and Applications (ICRERA), 2015, pp. 414-419.

[58] M. Vulovic, D. Boroyevich, and P. Mattavelli, "Digital gain-scheduled control of a high frequency parallel resonant DC-DC converter," in Proc. IEEE Applied Power Electronics Conference and Exposition (APEC) 27th Annual 2012, pp. 1814-1820.

[59] K. R. Sree and A. K. Rathore, "Impulse commutated zero-current switching currentfed push-pull converter: analysis, design, and experimental results," IEEE Trans. Ind. Electron., vol. 62, no. 1, pp. 363-370, Jan. 2015.

[60] J.-F. Chen, R.-Y. Chen, and T.-J. Liang, "Study and implementation of a singlestage current-fed boost PFC converter With ZCS for high voltage applications," IEEE Trans. Power Electron., vol. 23, no. 1, pp. 379-386, Jan. 2008.

[61] F. Carastro, A. Castellazzi, J. Clare, and P. Wheeler, "High-efficiency highreliability pulsed power converters for industrial processes," IEEE Trans. Power Electron., vol. 27, no. 1, pp. 37-45, Jan. 2012.

[62] T. B. Soeiro, J. Muhlethaler, J. Linner, P. Ranstad, and J. W. Kolar, "Automated design of a high-power high-frequency LCC resonant converter for electrostatic precipitators," IEEE Trans. Ind. Electron., vol. 60, no. 11, pp. 4805-4819, Nov. 2013.

[63] S.-H. Ahn, H.-J. Ryoo, G. J. -W., and S.-R. Jang, "Low-ripple and high-precision high-voltage DC power supply for pulsed power applications," IEEE Trans. Plasma Sci., vol. 42, no. 10, pp. 3023-3033, Oct. 2014.

[64] J. A. Martin-Ramos, P. J. V. Saiz, A. M. Pernia, J. Diaz, and J. A. Martinez, "Optimal control of a high-voltage power supply based on the PRC-LCC topology with a capacitor as output filter," IEEE Trans. Ind. Appl., vol. 49, no. 5, pp. 23232329, Sep./Oct. 2013.

[65] R. Beiranvand, B. Rashidian, M. R. Zolghadri, and S. M. H. Alavi, "Using LLC resonant converter for designing wide-range voltage source," IEEE Trans. Ind. Electron., vol. 58, no. 5, pp. 1746-1756, May 2011.

[66] I.-H. Cho, Y.-D. Kim, and G.-W. Moon, "A half-bridge LLC resonant converter adopting boost PWM control scheme for hold-Up state operation," IEEE Trans. Power Electron, vol. 29, no. 2, pp. 841-850, Feb. 2014.

[67] J.-W. Kim and G.-W. Moon, "A new LLC series resonant converter with a narrow switching frequency variation and reduced conduction losses," IEEE Trans. Power Electron., vol. 29, no. 8, pp. 4278-4287, Aug. 2014.

[68] W. Li, Q. Luo, Y. Mei, S. Zong, X. He, and C. Xia, "Flying-capacitor-based hybrid LLC converters with input voltage autobalance ability for high voltage applications," IEEE Trans. Power Electron., vol. 31, no. 3, pp. 1908-1920, Mar. 2016.

[69] J. Deng, S. Li, S. Hu, C. C. Mi, and R. Ma, "Design methodology of LLC resonant converters for electric vehicle battery chargers," IEEE Trans. Veh. Technol., vol. 63, no. 4, pp. 1581-1592, May 2014.

[70] R. Yang, H. Ding, Y. Xu, L. Yao, and Y. Xiang, “An analytical steady-state model of LCC type series-parallel resonant converter with capacitive output filter," IEEE Trans. Power Electron., vol. 29, no. 1, pp. 328-338, Jan. 2014. 
[71] X. Fang, H. Hu, Z. J. Shen, and I. Batarseh, "Operation mode analysis and peak gain approximation of the LLC resonant converter," IEEE Trans. Power Electron., vol. 27, no. 4, pp. 1985-1995, Apr. 2012.

[72] W. J. Lee, C. E. Kim, G. W. Moon, and S. K. Han, "A new phase-shifted full-bridge converter with voltage-doubler-type rectifier for high-efficiency PDP sustaining power module," IEEE Trans. Ind. Electron., vol. 55, no. 6, pp. 2450-2458, Jun. 2008.

[73] W. J. Cha, J. M. Kwon, and B. H. Kwon, "Highly efficient asymmetrical PWM fullbridge converter for renewable energy sources," IEEE Trans. Ind. Electron., vol. 63, no. 5, pp. 2945-2953, 2016.

[74] V. Kinnares and P. Hothongkham, "Circuit analysis and modeling of a phaseshifted pulsewidth modulation full-bridge-inverter-fed ozone generator with constant applied electrode voltage," IEEE Trans. Power Electron., vol. 25, no. 7, pp. 1739-1752, Jul. 2010.

[75] M. Yu, D. Sha, and X. Liao, "Hybrid phase shifted full bridge and LLC half bridge DC-DC converter for low-voltage and high-current output applications," IET Power Electron., vol. 7, no. 7, pp. 1832-1840, 2014.

[76] X. Liu et al., "Novel dual-phase-shift control with bidirectional inner phase shifts for a dual-active-bridge converter having low surge current and stable power control," IEEE Trans. Power Electron., vol. 32, no. 5, pp. 4095-4106, May 2017.

[77] J. Zhang, H. Zeng, and X. Wu, "An adaptive blanking time control scheme for an audible noise-free quasi-resonant flyback converter," IEEE Trans. Power Electron., vol. 26, no. 10, pp. 2735-2742, Oct. 2011.

[78] Y. C. Kang, C. C. Chiu, M. Lin, C. P. Yeh, J. M. Lin, and K. H. Chen, "Quasiresonant control with a dynamic frequency selector and constant current startup technique for $92 \%$ peak efficiency and $85 \%$ light-load efficiency flyback converter," IEEE Trans. Power Electron., vol. 29, no. 9, pp. 4959-4969, Sep. 2014.

[79] J.-P. Hong and G.-W. Moon, "A digitally controlled soft valley change technique for a flyback converter," IEEE Trans. Ind. Electron., vol. 62, no. 2, pp. 966-971, Feb. 2015.

[80] C. Y. Oh, D. H. Kim, D. G. Woo, W. Y. Sung, Y. S. Kim, and B. K. Lee, "A highefficient nonisolated single-stage on-board battery charger for electric vehicles," IEEE Trans. Power Electron., vol. 28, no. 12, pp. 5746-5757, Dec. 2013.

[81] V. L. Tran, N. T. Tran, S. H. Yu, Y. Park, and W. Choi, "Design of a nonisolated fuel cell boost charger for lithium polymer batteries with a low output ripple," IEEE Trans. Energy Convers., vol. 30, no. 2, pp. 605-614, Jun. 2015.

[82] N. O. Sokal and R. Redl, "Control algorithms and circuit designs for optimal flyback-charging of an energy-storage capacitor (e.g., for flash lamp or defibrillator)," IEEE Trans. Power Electron., vol. 12, no. 5, pp. 885-894, Sep. 1997.

[83] C.-H. Lin, M.-H. Hung, C.-Y. Ho, and C.-M. Wang, "High voltage pulse power supply for driving tubular field emission lamp based on flyback topology," in Proc. IEEE TENCON Region 10 Conf. 2011, pp. 898-902.

[84] G. C. Christidis, A. C. Nanakos, and E. C. Tatakis, "Hybrid discontinuous/boundary conduction mode of flyback microinverter for AC-PV modules," IEEE Trans. Power Electron., vol. 31, no. 6, pp. 4195-4205, Jun. 2016.

[85] K. I. Hwu and W. Z. Jiang, "Isolated step-up converter based on flyback converter and charge pumps," IET Power Electron., vol. 7, no. 9, pp. 2250-2257, Feb. 2014.

[86] Z. Zhang and L. Zhou, "Analysis and design of isolated flyback voltage-multiplier converter for low-voltage input and high-voltage output applications," IET Power Electron., vol. 6, no. 6, pp. 1100-1110, Mar. 2013.

[87] F. A. Dragonas, G. Neretti, P. Sanjeevikumar, and G. Grandi, "High-voltage highfrequency arbitrary waveform multilevel generator for DBD plasma actuators," IEEE Trans. Ind. Appl., vol. 51, no. 4, pp. 3334-3342, Jul./Aug. 2015. 
[88] C. L. Remes, M. B. Rosa, and S. V. G. Oliveira, "A two-switch forward converter application for battery charging," in Proc. IEEE 13th Brazilian Power Electron. Conf. and 1st Southern Power Electron. Conf. (COBEP/SPEC), 2015, pp. 1-6.

[89] Y. Yu, S. Yang, J. Bao, and S. Cheng, "Research on car-carried charger of polymer lithium battery used in electric vehicle," in Proc. Int. 7th Power Electron. Motion Control Conf. (IPEMC), 2012, vol. 3, pp. 1536-1539.

[90] L. M. Redondo and J. F. Silva, "Flyback versus forward switching power supply topologies for unipolar pulsed-power applications," IEEE Trans. Plasma Sci., vol. 37, no. 1, pp. 171-178, Jan. 2009.

[91] J.-H. Lee, J.-H. Park, and J. H. Jeon, "Series-connected forward-flyback converter for high step-up power conversion," IEEE Trans. Power Electron., vol. 26, no. 12, pp. 3629-3641, Dec. 2011.

[92] C. Lin, H. Haibing, Z. Qian, A. Amirahmadi, and I. Batarseh, "A boundary-mode forward-flyback converter with an efficient active LC snubber circuit," IEEE Trans. Power Electron., vol. 29, no. 6, pp. 2944-2958, Jun. 2014.

[93] G. Spiazzi, P. Mattavelli, and A. Costabeber, "High step-up ratio flyback converter with active clamp and voltage multiplier," IEEE Trans. Power Electron., vol. 26, no. 11, pp. 3205-3214, Nov. 2011.

[94] M. A. Rezaei, L. Kui-Jun, and A. Q. Huang, "A high-efficiency flyback microinverter with a new adaptive snubber for photovoltaic applications," IEEE Trans Power Electron., vol. 31, no. 1, pp. 318-327, Jan. 2016.

[95] S. H. Ahn, H. J. Ryoo, J. W. Gong, and S. R. Jang, "Design and test of a 35-kJ/s high-voltage capacitor charger based on a delta-connected three-phase resonant converter," IEEE Trans. Power Electron., vol. 29, no. 8, pp. 4039-4048, Aug. 2014.

[96] M. Uno and A. Kukita, "Double-switch equalizer using parallel- or series-parallelresonant inverter and voltage multiplier for series-connected supercapacitors," IEEE Trans. Power Electron., vol. 29, no. 2, pp. 812-828, Feb. 2014.

[97] J.-W. Gong, S.-H. Ahn, H.-J. Ryoo, and S.-R. Jang, "Comparison of DCM and CCM operated resonant converters for high-voltage capacitor charger," in Proc. IEEE 39th Ind. Electron. Soc.( IECON) Annual Conf. 2013, 2013, pp. 532-537.

[98] N. Shafiei and M. Ordonez, "Improving the regulation range of EV battery chargers with L3C2 resonant converters," IEEE Trans. Power Electron., vol. 30, no. 6, pp. 3166-3184, Jun. 2015.

[99] M. Borage, S. Tiwari, and S. Kotaiah, "Analysis and design of an LCL-T resonant converter as a constant-current power supply," IEEE Trans. Ind. Electron., vol. 52, no. 6, pp. 1547-1554, Dec. 2005.

[100] H. Sheng et al., "Design and implementation of a high power density three-level parallel resonant converter for capacitor charging pulsed-power supply," IEEE Trans. Plasma Sci., vol. 39, no. 4, pp. 1131-1140, Apr. 2011.

[101] W. Li, H. Zhao, S. Li, J. Deng, T. Kan, and C. C. Mi, "Integrated LCC compensation topology for wireless charger in electric and plug-in electric vehicles," IEEE Trans. Ind. Electron., vol. 62, no. 7, pp. 4215-4225, Jul. 2015.

[102] T. Xing and R. Xinbo, "Optimal design of DCM LCC resonant converter with inductive filter based on mode boundary map," IEEE Trans. Power Electron., vol. 30, no. 8, pp. 4144-4155, Aug. 2015.

[103] X. Y. and P. K. Jain, "A forward converter topology employing a resonant auxiliary circuit to achieve soft switching and power transformer resetting," IEEE Trans. Ind. Electron., vol. 50, no. 1, pp. 132-140, Feb. 2003.

[104] M. Khodabakhshian, E. Adib, and H. Farzanehfard, "Forward-type resonant bidirectional DC-DC converter," IET Power Electron., vol. 9, no. 8, pp. 1753-1760, 2016.

[105] Y. Gu, Z. Lu, Z. Qian, X. Gu, and L. Hang, "A Novel ZVS resonant reset dual switch forward DC-DC converter," IEEE Trans. Power Electron., vol. 22, no. 1, pp. 96-103, Jan. 2007. 
[106] M. Kim and S. Choi, "A fully soft-switched single switch isolated DC-DC converter," IEEE Trans. Power Electron., vol. 30, no. 9, pp. 4883-4890, Sep. 2015.

[107] J.-M. Kwon, W.-Y. Choi, and B.-H. Kwon, "Single-switch quasi-resonant converter," IEEE Trans. Ind. Appl., vol. 56, no. 4, pp. 1158-1163, Apr. 2009.

[108] H. Keyhani and H. A. Toliyat, "Partial-resonant buck-boost and flyback DC-DC converters," IEEE Trans. Power Electron., vol. 29, no. 8, pp. 4357-4365, Aug. 2014.

[109] P. Ki-Bum, K. Chong-Eun, M. Gun-Woo, and Y. Myung-Joong, "PWM resonant single-switch isolated converter," IEEE Trans. Power Electron., vol. 24, no. 8, pp. 1876-1886, Aug. 2009.

[110] W.-J. Cha, Y.-W. Cho, J.-M. Kwon, and B.-H. Kwon, "Single power-conversion LED backlight driving system with high power factor control," J. Display Technol., vol. 10, no. 5, pp. 407-413, May 2014.

[111] A. Emrani, E. Adib, and H. Farzanehfard, "Single-switch soft-switched isolated DC-DC converter," IEEE Trans. Power Electron., vol. 27, no. 4, pp. 1952-1957, Apr. 2012.

[112] P. Thummala, D. Maksimovic, Z. Zhang, and M. Andersen, "Digital control of a high voltage $(2.5 \mathrm{kV})$ bidirectional flyback DC-DC converter for driving a capacitive incremental actuator," IEEE Trans. Power Electron., vol. PP, no. 99, pp. 1-1, Jan. 2016.

[113] H. Wang and F. Blaabjerg, "Reliability of capacitors for DC-link applications in power electronic converters-an overview," IEEE Trans. Ind. Appl., vol. 50, no. 5, pp. 3569-3578, Sep./Oct. 2014.

[114] J. Liu and N. Henze, "Reliability consideration of low-power grid-tied inverter for photovoltaic application," in Proc. 24th European Photovoltaic Solar Energy Conference, Hamburg, Germany, 2009, pp. 21-25.

[115] M. B. Shadmand and R. S. Balog, "A finite-element analysis approach to determine the parasitic capacitances of high-frequency multiwinding transformers for photovoltaic inverters," in Proc. IEEE Power and Energy Conference at Illinois (PECI), 2013, pp. 114-119.

[116] C. W. T. McLyman, Transformer and inductor design handbook, 4th ed. CRC press, 2011.

[117] N. Shafiei, M. Pahlevaninezhad, H. Farzanehfard, A. Bakhshai, and P. Jain, "Analysis of a fifth-order resonant converter for high-voltage DC power supplies," IEEE Trans. Power Electron., vol. 28, no. 1, pp. 85-100, Jan. 2013.

[118] N. Shafiei, M. Pahlevaninezhad, H. Farzanehfard, and S. R. Motahari, "Analysis and implementation of a fixed-frequency LCLC resonant converter with capacitive output filter," IEEE Trans. Ind. Appl., vol. 58, no. 10, pp. 4773-4782, Oct. 2011.

[119] D. Thenathayalan and J.-H. Park, "Wide-air-gap transformer model for the designoriented analysis of contactless power converters," IEEE Trans. Ind. Electron., vol. 62, no. 10, pp. 6345-6359, Oct. 2015.

[120] B. Chen et al., "A high-efficiency MOSFET transformerless inverter for nonisolated microinverter applications," IEEE Trans. Power Electron., vol. 30, no. 7, pp. 3610-3622, Jul. 2015.

[121] Z. Ouyang, O. C. Thomsen, and M. A. E. Andersen, "Optimal design and tradeoff analysis of planar transformer in high-Power DC-DC converters," IEEE Trans. Ind. Electron., vol. 59, no. 7, pp. 2800-2810, Jul. 2012.

[122] J. Park et al., "Quasi-resonant (QR) controller with adaptive switching frequency reduction scheme for flyback converter," IEEE Trans. Ind. Electron., vol. 63, no. 6, pp. 3571-3581, Jun. 2016. 